\title{
Classification and Analysis of Management and Marketing Data
}

Statistical Applications for Strategic Planning

\author{
Dissertation \\ Presented for the Degree of Doctor of Philosophy \\ at the Faculty of Economics and Business Administration \\ of the Georg-August University of Göttingen
}

by

Tina M. Facca

from

Ohio, United States of America

Göttingen, 2010 
First Examiner: $\quad$ Prof. Dr. Walter Zucchini

Second Examiner: Prof. Dr. Yasemin Boztuğ

Third Examiner: $\quad$ Prof. Dr. Stefan Sperlich 


\section{Contents}

Introduction ............................................................

Classification and Analysis of a Job-Seeking Market

Part 1 Dataset with numerical and graphical description

Section $1.1 \quad$ Motivation and literature................................ 13

Section $1.2 \quad$ Loyalty scores........................................ 18

Section 1.3 Description of the dataset............................. 20

Section 1.4 General problem...................................... 28

Section 1.5 Problem of classification by subclass..................... 34

Section 1.6 Conclusion - Better classification method needed ............ 46

Part 2 Classifying job seeking status based on sub-groups

Section $2.1 \quad$ Definition of loyalty score ........................... 47

Section 2.2 Descriptive statistics and graphic normality check of

loyalty score and its components..........................6 65

Section 2.3 Descriptive statistics of factors by subclass.................70

Section 2.4 Conditional probability given loyalty score.............. 84

Section 2.5 Classification based on intervals - cluster analysis......... 88

Section 2.6 Classification using discriminant analysis................ 94

Section 2.7 Building a generalized additive model to predict job-seeking status.............................................. 117

Section 2.8 Summary, limitations and future research................. 125

References Classification and analysis of a job-seeking market........ 127

\section{Classification and Analysis of College Student Leadership Data}

$\underline{\text { Part } 1 \text { Dataset with numerical and graphical description }}$

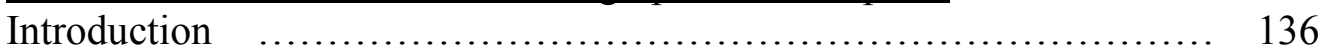

Section 1.1 College student leadership literature.................... 137

Section 1.2 Description of the dataset .......................... 151

Section 1.3 General problem - measuring leadership.................. 157

Section 1.4 Problem of classification - consciousness constructs........ 160

Part 2 Classifying college student leadership based on sub-groups

Section 2.1 Reliability of constructs and factor analysis.............. 173

Section 2.2 Classification by discriminant analysis.................... 176

Section 2.3 Cluster analysis to segment leadership behaviors........... 182

Section 2.4 Summary and future research.......................... 193

References Classification and analysis of College student leadership

data................................................ 196 


\section{Classification and Analysis of Franchise Resource Data}

Part 1 Dataset with numerical and graphical description

Section 1.1 Franchising market - issues, constraints and literature..... 204

Section 1.2 Description of the dataset............................ 211

Section 1.3 General problem - where to focus franchisor's resources... 219

Section 1.4 Problem of classification by tenure and revenue segments.. 221

Part 2 Classifying franchisees based on sub-groups

Section 2.1 Classification of franchisees using discriminant analysis... 229

Section 2.2 Summary - Classification and analysis of franchise data... 239

References Classification and analysis of franchise resource data..... 241

\section{APPENDICES}

Appendix A Job-Seeking Market - Section 2.3 Kruskal-Wallis test..... 243

Appendix B Job-Seeking Market - Section 2.6 validation test results

from discriminant analysis

\section{List of abbreviations}

SUPV - supervisor

COMP - company

OPPTY - opportunity

QWL - quality of work-life

PCA - principle components analysis

GAM - generalized additive model(s)

HERI - Higher Education Research Institute

EI - Emotional intelligence

EILI - Emotional intelligence leadership inventory 


\section{List of Figures}

Classification and Analysis of a Job-Seeking Market

Figure 1.3.1 Respondents' employment status..................... 20

Figure 1.3.2 Respondents' job seeking status....................... 21

Figure 1.3.3 Respondents' level of employment..................... 21

Figure 1.3.4 Respondents' most recent job change..................... 22

Figure 1.3.5 Respondents' salary level............................. 23

Figure 1.3.6 Respondents' industries represented.................... 23

Figure 1.3.7 Respondents' gender............................... 24

Figure 1.3.8 Respondents' age group.............................. 24

Figure 1.3.9 Respondents' geographic locations within United States 24

Figure 1.3 .10 Respondents' ethnicity................................ 25

Figure 1.3.11 Respondents' education................................ 25

Figure 1.5.1 Variables listed inside arrows -significant differences .... 37

Figure 1.5.2 Non-seeker vs. Seeker - significant differences.......... 39

Figure 1.5.3 Non-seeker vs. Passive - significant differences.......... 41

Figure 1.5.4 Passive vs. Seeker - significant differences............... 43

Figure 2.1.1 Component plot in rotated space........................ 51

Figure 2.1.2 Cattell screeplot..................................... 60

Figure 2.2.1 Supervisor not normally distributed.................... 66

Figure 2.2 .2 Company not normally distributed....................... 66

Figure 2.2.3 Opportunity not normally distributed $\ldots \ldots \ldots \ldots \ldots \ldots \ldots \ldots 67$

Figure 2.2.4 Quality of work-life not normally distributed............. 67

Figure 2.2.5 Loyalty score not normally distributed ................. 68

Figure 2.3.1 Seeker - supervisor not normally distributed ........... 72

Figure 2.3.2 Seeker - company not normally distributed ............. 72

Figure 2.3.3 Seeker - opportunity not normally distributed .......... 72

$\begin{array}{lll}\text { Figure 2.3.4 Seeker - quality of work-life not normally distributed... } & 72\end{array}$

Figure 2.3.5 Seeker - loyalty score not normally distributed ......... 73

Figure 2.3.6 Passive - supervisor not normally distributed ........... 74

Figure 2.3.7 Passive - company not normally distributed ............ 74

Figure 2.3.8 Passive - opportunity not normally distributed ......... 74

Figure 2.3.9 Passive - quality of work-life not normally distributed ... 74

Figure 2.3.10 Passive- loyalty score not normally distributed .......... 75

Figure 2.3.11 Non-seeker - supervisor not normally distributed ....... 76

Figure 2.3.12 Non-seeker - company not normally distributed ......... 76

Figure 2.3.13 Non-seeker - opportunity not normally distributed ...... 77

Figure 2.3.14 Non-seeker - quality work life not normally distributed $\quad 77$

Figure 2.3.15 Non-seeker - loyalty score not normally distributed..... 71

Figure 2.3.16 Boxplots for supervisor by subclass.................. 80

Figure 2.3.17 Boxplots for company by subclass ................... 81

Figure 2.3.18 Boxplots for opportunity by subclass................... 81

Figure 2.3.19 Boxplots for quality of work-life by subclass........... 82

Figure 2.3.20 Boxplots for loyalty score by subclass................. 83 
Figure 2.6.1 Two-group discriminant function....................... 96

Figure 2.6.2 Solid lines representing fitted LDA boundaries............ 98

Figure 2.6.3 Separation produced by first discriminant function......... 113

Figure 2.6.4 Separation produced by second discriminant function........ 114

Figure 2.7.1 Functions at three degrees of freedom ..................... 121

Figure 2.7.2 Varying each factor while holding others constant; three degrees of freedom..................................... 122

Figure 2.7.3 Functions at two degrees of freedom ..................... 123

Figure 2.7.4 Varying each factor while holding others constant; two degrees of freedom........................................124

Classification and Analysis of College Student Leadership Data

Figure 1.2.1 Respondent gender................................... 154

Figure 1.2.2 Respondent ethnicity................................. 154

Figure 1.2.3 Respondent class rank................................... 154

Figure 1.2.4 Respondent age category.............................. 155

Figure 1.2.5 Respondent participation in student organizations.......... 155

Figure 1.2.6 Respondent in leadership role........................... 155

Figure 1.2.7 Respondent leadership retreats.......................... 156

Figure 1.4.1 Construct score comparisons by level....................161

Figure 1.4.2 Comparison by involvement.............................. 166

Figure 1.4.3 Max leaders vs. base on construct scores.................. 167

Figure 1.4.4 Men in student organizations............................ 169

Figure 1.4.5 Women in student organizations............................169

Figure 2.3.1 Cluster Profiles...................................... 186

Figure 2.3.2 Categorical discriminators for less involved, less others-oriented group ........................... 187

Figure 2.3.3 Level of involvement differentiating third cluster ........ 188

Figure 2.3.4 Discriminant scores for each cluster from first discriminant function.......................................... 189

Figure 2.3.5 Second discriminant function......................... 191

Classification and Analysis of Franchise Resource Data

Figure 1.2.1 Franchisee tenure distribution.......................... 212

Figure 1.2.2 Franchisee sales distribution............................... 212

Figure 1.2.3 Number of employees in franchisee office................. 213

Figure 1.2.4 Franchisee satisfaction - areas of service.................. 213

Figure 1.2.5 Franchisee satisfaction - programs and services ........... 214

Figure 1.2.6 Franchisee satisfaction - field consulting services .......... 214

Figure 1.2.7 Franchisee satisfaction - public relations services ..........215

Figure 1.2.8 Franchisee satisfaction - franchise information website.... 215

Figure 1.2.9 Franchisee awareness, usage, intent to use training ....... 216

Figure 1.2.10 Franchisee effectiveness, value perceptions - training...... 216 
Figure 1.2.11 Franchisee awareness, usage and intent to use meetings.... 217

Figure 1.2.12 Franchisee effectiveness, value perceptions - meetings.....2217

Figure 1.2.13 Franchisee satisfaction by revenue segment and tenure..... 218

Figure 1.4.1 Franchisee segments, percentage in segment, satisfaction... 221

Figure 1.4.2 How to read upcoming figures - requirements by segment.. 222

Figure 1.4.3 Franchisor resources required Low tenure/high revenue.... 223

Figure 1.4.4 Franchisor resources required Low tenure/low revenue...... 224

Figure 1.4.5 Franchisor resources required High tenure/high revenue ....226

Figure 1.4.6 Franchisor resources required High tenure/low revenue..... 227

Figure 2.1.1 Discriminant scores for low tenure segment based on group

classification....................................... 237

Figure 2.1.2 Discriminant scores for high tenure segment based on group

classification...................................... 238 


\section{List of Tables}

Classification and Analysis of a Job-Seeking Market

Table 1.3.1 Current company questions......................... 26

Table 1.3.2 External influence questions.......................... 26

Table 1.3.3 Current supervisor questions......................... 27

Table 1.3.4 Additional company related questions .................. 27

Table 1.3.5 Job-seeking status question and response categories...... 27

Table 1.4.1 Variables in the loyalty regression model............... 33

Table 1.5.1 Significant Differences -Non-seekers vs. Seeker......... 39

Table 1.5.2 Significant Differences - Non-seekers vs. Passives....... 41

Table 1.5.3 Significant Differences - Seekers vs. Passives............. 44

Table 1.5.4 Significant Differences - likelihood to recommend........ 45

Table 1.5.5 Means and standard deviation by category.............. 45

Table 2.1.1 Principle component analysis; total variance explained ... 51

Table 2.1.2 Variables loading on each component..................... 52

Table 2.1.3 Factor reliability...................................... 52

Table 2.1.4 Comparison of variances full dataset vs. subclasses........ 56

Table 2.1.5 Levene's test - variances unequal....................... 57

Table 2.1.6 Component scores used as weights..................... 58

Table 2.1.7 Indicator variable loadings; rotated component matrix...... 59

Table 2.1.8 Composition of final factors ........................... 61

Table 2.1.9 Mean scores; factors by seeking status.................. 61

Table 2.2.1 Shapiro-Wilks' test for normality factors and loyalty score. 66

Table 2.2.2 Descriptive statistics for factors and loyalty score......... 67

Table 2.2.3 Skewness and kurtosis for factors and loyalty score........ 68

Table 2.3.1 Self-reported seeking status categories....................70

Table 2.3.2 Shapiro-Wilks' test for normality of loyalty by subclass... 71

Table 2.3.3 Descriptive statistics for seekers......................... 73

Table 2.3.4 Skewness and kurtosis for seekers..........................73

Table 2.3.5 Descriptive statistics for passives........................ 75

Table 2.3.6 Skewness and kurtosis for passives...................... 76

Table 2.3.7 Descriptive statistics non-seekers...................... 78

Table 2.3.8 Skewness and kurtosis for non-seekers....................78

Table 2.3.9 All subclasses differ significantly on Supervisor factor..... 78

Table 2.3.10 All subclasses differ significantly on Company factor...... 79

Table 2.3.11 No significant difference between passives and seekers on Opportunity factor.......................................79

Table 2.3.12 No significant difference between passives and non-seekers on Quality of Work Life.............................. 79

Table 2.3.13 Significant differences on loyalty score for all subclasses. 79

Table 2.4.1 Percentage correctly classified into each interval.......... 85

Table 2.4.2 Percentage correctly classified - Equal subclasses.......... 86

Table 2.4.3 Percentage correctly classified - All data................ 86

Table 2.4.4 Percentage correctly classified - Data inspection intervals 87

Table 2.5.1 K-means clustering of Loyalty Scores.................. 91 
Table 2.5.2 K-means clustering of Loyalty Scores, based on factors ... 92

Table 2.5.3 K-means clustering of Loyalty Scores, 3 clusters........... 92

Table 2.6.1 Shapiro-Wilks' test of normality; factors \& interactions..... 101

Table 2.6.2 Significant differences seekers vs. passives on 4 factors, 6

interactions........................................... 102

Table 2.6.3 Significant differences passives vs. non-seekers on 4 factors, 6 interactions.......................................... 103

Table 2.6.4 Validation of significance of discriminant functions.........106

Table 2.6.5 Standardized canonical discriminant function coefficients.. 106

Table 2.6.6 Structure matrix........................................ 107

Table 2.6.7 Stepwise results - variables in final discriminant function.. 108

Table 2.6.8 Significant differences between mean discriminant scores

for seekers vs. passives................................ 109

Table 2.6.9 Significant differences between mean discriminant scores

for passives vs. non-seekers............................... 109

Table 2.6.10 Fisher's linear discriminant functions.....................110

Table 2.6.11 Average correct classifications from 3 validation tests -

Pooled covariance matrices...............................111

Table 2.6.12 Average correct classifications from 3 validation tests -

Separate covariance matrices...............................112

Table 2.6.13 Classification matrix validated with Press' Q statistic....... 113

Table 2.6.14 Seeker discriminant score descriptive statistics.............115

Table 2.6.15 Passive discriminant score descriptive statistics............115

Table 2.6.16 Non-seeker discriminant score descriptive statistics........ 115

Classification and Analysis of College Student Leadership Data

Table 1.2.1 Leadership behavior questions......................... 151

Table 1.2.2 Consciousness of Context - Descriptive Statistics.......... 152

Table 1.2.3 Consciousness of Self -Descriptive Statistics.............. 153

Table 1.2.4 Consciousness of Others - Descriptive Statistics............ 153

Table 1.4.1 Levels of consciousness constructs........................160

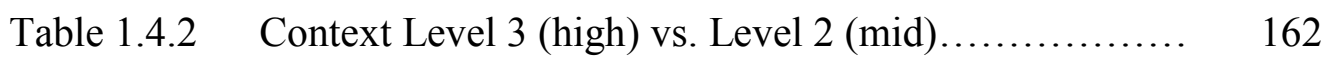

Table 1.4.3 Context Level 2 (mid) vs. Level 1 (low)............... 162

Table 1.4.4 Self Level 3 (high) vs. Level 2 (mid).................... 163

Table 1.4.5 Self Level 2 (mid) vs. Level 1 (low).................... 163

Table 1.4.6 Others Level 3 (high) vs. Level 2 (mid).................. 164

Table 1.4.7 Others Level 2 (mid) vs. Level 1 (low)................. 165

Table 1.4.8 Max leader vs. base - significant differences.................. 168

Table 1.4.9 Gender differences for individual measures................. 170

Table 1.4.10 Gender differences for individual measures; involvement... 172

Table 2.1.1 Variables comprising consciousness of context construct....173

Table 2.1.2 Variables comprising consciousness of self construct....... 173

Table 2.1.3 Variables comprising consciousness of others construct... 174

Table 2.1.4 Reliability of factors ............................... 174

Table 2.1.5 Rotated component score matrix - five factors........... 175 
Table 2.2.1 Stepwise results - discriminatory variables, max-leaders vs. others........................................ 178

Table 2.2.2 Stepwise discriminant analysis distinguishing gender....... 179

Table 2.2.3 Stepwise discriminant analysis distinguishing gender among max-leaders.................................... 180

Table 2.2.4 Male category, discriminating max-leaders from base...... 181

Table 2.2.5 Female category, discriminating max-leaders from base..... 181

Table 2.3.1 Variables most significantly contributing to third cluster membership........................................... 187

Table 2.3.2 Variables most significantly contributing to first cluster membership........................................... 189

Table 2.3.3 Discriminant analysis classification results using cluster membership as grouping variable........................ 192

Classification and Analysis of Franchise Resource Data

Table 1.4.1 Significant differences - low tenure high revenue (Stars) and low tenure low revenue (Brats) segments........... 225

Table 1.4.2 Significant differences - high tenure high revenue segment and high tenure low revenue segment..................... 227

Table 2.1.1 Low tenure franchisees - classification with significantly different variables......................................... 230

Table 2.1.2 Low tenure franchisees - structure matrix................ 230

Table 2.1.3 High tenure franchisee segments - classification with significantly different variables......................... 232

Table 2.1.4 High tenure franchisee segments - structure matrix....... 232

Table 2.1.5 Low tenure franchisees - final discriminant function ....... 234

Table 2.1.6 Low tenure segments using six variables, classification..... 234

Table 2.1.7 High tenure franchisees - final discriminant function ...... 235

Table 2.1.8 High tenure segments using eight variables, classification.. 236 


\section{Introduction}

This work investigates and provides examples of effective methods of statistical analysis for strategic decision making, specifically in managerial and marketing applications. The particular motivation is to bridge the gap between statistics and marketing management by presenting statistical methods that are helpful in classifying market or management segments. The contributions made are innovative approaches to applying rather traditional statistical methods to solve varied managerial and marketing issues. Three datasets are presented and analyzed, and statistical methods are applied to solve three different management and marketing problems. While the methods are not necessarily new, their application in management decision making is unique given the contexts in which they are applied.

The work is presented in three parts, each addressing a specific management issue. Varied analytical methods are applied, and a distinct contribution is made, using the classification techniques determined as most effective given the application.

The first portion investigates job-seeking behaviors and aims to classify employees as either currently seeking a new position, passive, or not seeking a new position outside the company. Knowledge of employees' seeking status provides management with a better understanding of the stability of the organization. The managerial challenge is to generate accurate classification without directly asking the employee about their job-seeking status, given that perceived risk is high, particularly for the employee. Difference analysis, factor analysis, discriminant analysis and generalized additive models are investigated and a method is created for determining the probability that an employee falls into each of the seeking status categories.

The second portion examines college student leadership behaviors. The aim is to provide a reliable self-assessment tool and understand the underlying constructs of college student leadership behaviors. Difference analysis, factor analysis, cluster analysis and discriminant analysis for validation are methods implemented. The contribution is a reliable and valid self-assessment tool to help students evaluate their current leadership behaviors and focus on behaviors that facilitate improvement of leadership skills. The self-assessment tool can be applied in managerial settings to help employees improve their leadership skills. 
The third portion addresses resource allocation in a business franchise. The issue addressed is determining the most effective allocation of franchisor resources to support franchisee revenue growth. The objective is to enable a franchisor to clearly differentiate between franchisees with the same level of tenure with the franchise, yet significantly different revenue results. Discriminant analysis enables the classification of franchisees into segments of revenue results based on their perceptions of effectiveness and value, satisfaction with, and intended future use of the services offered by the franchisor. The contribution is the development of a method to effectively allocate franchisor resources which mimics the resource utilization behaviors of the higher revenue-generating franchisees. 


\section{Classification and Analysis of a Job-Seeking Market}

\subsection{Motivation and Literature}

A method is sought that will enable the scoring of employees in a manner that reveals the probability of their job-seeking status as currently seeking, or passive in that the person would leave their current job given the right opportunity, or not seeking an alternate job at the time. The method should enable the identification of status without directly asking the employee. If the question of job-seeking status is asked by the employer, it is not likely that the employee would provide a truthful answer, particularly if passive or seeking. An analytical method is proposed that enables classification of seeking status based on a limited number of questions determined to yield the most information.

Classification and Analysis of Job-Seeking Market

This work will be presented in two parts. Part 1 will describe the dataset, with graphical and numerical support, followed by Part 2 which discusses how to classify job-seeking status based on the analysis of the sub-groups of seekers, passives, and non-seekers.

\section{Literature Discussion}

The industrial psychology literature delivers rich, relevant material regarding job satisfaction and turnover with roots in the 1950's. One consistently agreed upon element is that there is a negative relationship between job satisfaction and employee turnover (Brayfield \& Crockett, 1955; Vroom, 1964: Porter and Steers, 1973; Locke 1975). Given measurements of satisfaction, researchers should be able to effectively predict potential for turnover.

March and Simon's work (1958) suggested that attitudes toward a job should affect the decision to participate (i.e., stay in the organization) more than it affects job performance. Herzberg's job satisfaction factors and techniques from the mid-1960s focused on turnover versus performance. Then, in 1972, Atchison and Lefferts studied dissatisfaction patterns that make turnover likely using Herzberg's job satisfaction factors and techniques. They found that personality, the comparison standard, and perceptions of fairness or equity all helped to distinguish subjects who stayed in their organization versus those who terminated.

Porter and Steers (1973) suggested that future research focus on the psychology of the organizational withdrawal process because they believed 
that "expressed intention to leave" was the next logical step after dissatisfaction had been experienced.

In 1977, Mobley investigated this organizational withdrawal process, and highlighted variables that link job attitudes with actual turnover, finding that the job attitudes most directly related to withdrawal cognitions associated with decision to leave were only indirectly related to actual turnover behavior. In 1979 Mobley et al suggested that turnover was more than job satisfaction, and that researchers needed to look at more variables in a comprehensive attempt to identify a broad range of factors that can initiate desire to leave organization. They presented a conceptual model that identified a need to distinguish between present-oriented satisfaction and future oriented "attraction/expected utility" for both the current job versus alternatives. They further suggest the need for a mechanism to integrate aggregate-level research findings into an individual model of the turnover process (Mobley, et al, 1979).

Arnold and Feldman (1982) investigated and critiqued the existing models of turnover and their variations to create a new working model of actual turnover behavior. While this research is not directly aimed at developing another model of turnover behavior, a review of Arnold and Feldman's perceptions of then-extant research and models adds insight to the variations in causal ordering.

The Steers and Mowday (1981) model suggests the turnover process starts with job expectations and values, which are considered simultaneously; these lead to affective responses which in turn lead to intent to leave. Exogenous variables influencing job expectations and values include alternate job opportunities, economic and market conditions, and individual differences.

The Mobley et al (1979) version starts with individual values, which lead to affective responses. These in turn, lead to intent to search/quit simultaneously, yet with economic conditions and job expectations intervening between affective responses and intention to search and quit. This model may be more reflective of the currently troubled global economy. It should be noted however, that the severity of the current economic crisis was not in full effect at the time of data collection in early 2007.

Gerhart (1990) highlights the fact that voluntary turnover literature provides a recurring theme that the availability of alternative jobs influences turnover intentions and behaviors. March and Simon (1958) stated "under nearly all conditions the most accurate single predictor of labor turnover is the state of the economy...When jobs are plentiful, voluntary movement is high; when 
jobs are scarce, voluntary turnover is small (p. 100). Economic literature agrees that tight labor markets (i.e. plentiful jobs relative to seekers) suggest high turnover rates, whereas loose markets experience fewer transitions in the workforce. Unemployment is a useful measure of tightness (Ehrenberg \& Smith, 1982, p. 285).

March and Simon (1958) suggested that perceived ease of transition (or movement) interacts with desire to transition to influence turnover. More importantly these perceptions were suggested to be the result of general labor market conditions. Perceived ease and desirability of movement resulted in a push and pull model where dissatisfaction is more likely to push employee to consider job alternatives, and attractive alternatives would pull the employee toward transition. Again, such perceptions were suggested to be influenced by general market conditions.

Mobley's work with Horner and Hollingsworth (1978) suggests an alteration of the ordered turnover process: individual differences influence job satisfaction, and probability of finding alternatives (simultaneously) influences thinking of quitting, which in turn influences intention to search, which influences intention to leave, which results in turnover.

Evaluating the Mobley, Horner, Hollingsworth model, Miller, Katerberg, and Hulin (1979) found that the seven variables studied collapsed into four factors: withdrawal behavior (or turnover itself), withdrawal cognitions (intent to quit, intent to search, and thinking of quitting), job satisfaction and career mobility (age, tenure, probability of finding an acceptable alternative). This group suggested a more general model of career mobility which influences job satisfaction which can lead to withdrawal cognitions resulting in turnover.

Arnold and Feldman suggested the most powerful model of turnover behavior contained four significant individual predictor variables: tenure, job satisfaction, perceived job security and the intention to search for an alternative position. It is the intent to search, and the situation surrounding that intent, consideration, or lack of intent, that is the interest of the research herein.

Further, it is interesting to note other related research in the mid 70's, in which analytical activity in the field of organizational behavior takes similar approaches to those applied here, namely factor and discriminant analysis. Sims, Szilagyi, and Keller worked to improve an instrument for measuring job characteristics that could be applied to multiple organizations (Sims, et al, 1976). Similarly, the work to be presented here aims to construct and universal 
instrument, that effectively distinguishes job-seeking status as seeking, passive or not seeking.

Investigating external influences on one's decision to leave a company was suggested by Mobley, Griffith, Hand, and Meglino thirty years ago, as they discussed "Attraction and Expected Utility of Alternatives: Considering both satisfaction and attraction expected utility should increase our understanding and prediction of turnover intentions and behavior." (Mobley et al, 1979).

The idea of expected utility (as well as expected value) is common in economics and decision theory. As early as 1956, Blau, Gustad, Jessor, Parnes and Wilcox took an interdisciplinary approach to the evaluation of occupational alternatives considering such evaluation as the "individual's valuation of the rewards offered by different alternatives and his appraisal of his chances of being able to realize each of the alternatives" (Blau et al, 1956).

Supporting the suggestion of a need to identify employees in the passive state is Kirschenbaum and Weisberg's work (1994). They investigated the decision process associated with turnover and suggested a causal path in which "passive search occurs before the crystallization of a turnover intent, and after an intent has emerged, an active search begins." Further, they discuss the passive job search in which minimal effort is made to generate job market information. An important point presented by Kirschenbaum and Weisberg is that the passive's behavior is reflective of a free-market economy where one compares alternatives and evaluates self-worth in the labor market both within the organization and externally. They suggest that passive search "does not necessarily lead to any particular pattern of active search associated with turnover... acting only as a springboard for the varied perceived reactions to the environment." (Kirschenbaum and Weisberg, 1994).

It is precisely this "springboard" element that this research aims to analyze and subsequently utilize for classification. Given the catalysts that lead to transition out of the organization, it is during this passive state that employees are most vulnerable to recruiters' solicitation, friends' recommendations, and other employment-networking opportunities which represent a potential loss to the organization if the passive is in fact a valued employee.

The role of the supervisor in turnover intentions was investigated by DeConick and Stilwell (2001) who found that supervisor satisfaction had a direct influence on withdrawal cognitions. Brough and Frame (2004) found that supervisor support was a strong predictor of job satisfaction and also an indirect predictor of turnover intentions. Further, Eisenberger, Stinglahamber, Vandenberghe, Sucharski, and Rhoades (2002) found that supervisor support 
was negatively correlated with turnover (i.e., low supervisor support resulted in higher turnover intentions).

DeConick and Stilwell (2001) additionally investigated the role of the company in turnover behaviors with a variable referred to as organizational justice (Berkowitz et al, 1987; Folger and Cropanzano, 1998; Folger and Konovsky, 1989; Greenberg, 1990; Sweeney and McFarlin, 1993). They suggest the extreme importance of organizational justice because of its link to employees' job satisfaction, organizational commitment, and their likelihood of searching for another job. Further, DeConick and Stilwell found that the supervisor acts as a mediator between employees' perceptions of the company (organizational justice) and their commitment to the organization. Employee perceptions of their supervisor can assist management in understanding variations in organizational commitment (DeConick and Stilwell, 2001).

The impact of work-life benefits on job-pursuit intentions was studied by Cater and Buffardi (2004) who found benefits such as schedule flexibility and dependent care assistance to contribute uniquely to predicting anticipated organizational support and job pursuit intentions. Beauregard and Henry (2009) have recently tried to make the link between work-life balance and organizational performance. They concluded that there is insufficient evidence to support the notion that work-life practices enhance performance by means of reduced work-life conflict. However, they suggest a modification to the business case for work-life balance practices which reflects additional impacts of such practices on organizations.

This work validates the notion that perceptions of the company, perceptions of the supervisor, and to a lesser extent the quality of work-life and alternate opportunities, serve to discriminate between seekers, passives and nonseekers. The unique approach to analysis and classification that will be discussed here appears to be consistent with the requests for future research found in the literature.

The research instrument, methodology, and implementation process presented here were designed, and data collection implemented, prior to establishing the intent for utilizing the data for advanced analysis and classification as in this research application. Experience and intuition guided the process somewhat fortuitously as four reliable underlying constructs were ultimately uncovered which include satisfaction with current supervisor, satisfaction with current company, the influence of external opportunities, and the influence of an improved quality of work life. These constructs are used to discern the seeking status of employees within an organizational setting. 


\subsection{Loyalty Scores}

The concept of measuring loyalty to a brand, product, or in our case, an organization, is relatively straightforward. One common method employed by market researchers is to ask the question, "How likely are you to recommend this product or service to friends or family." Typically, the researcher generates a score from the respondent, for example, on a scale from $1=$ not at all likely to $10=$ very likely. One can build a model based on stepwise multiple regression analysis which identifies the independent variables that serve as significant predictors of likelihood to recommend, or loyalty.

In turn, an organization can focus on promoting improvements in the variables that drive loyalty. This work is typically recommended by researchers, communicated to organizational leadership by marketers, implemented by operations personnel, and measured again by researchers to assess significance of change.

In section 1.4 the general problem of loyalty and its objective measurement is discussed in more detail, but here the use of scores for "likelihood to recommend" as a measure of loyalty are highlighted.

In recent years, the concept of measuring loyalty based on likelihood to recommend has grown in popularity thanks to Frederick F. Reichheld. First introduced by Reichheld in a 2003 Harvard Business Review article, "The One Number You Need to Grow," the net promoter score has been widely adopted as a customer loyalty metric that proponents suggest far outweighs the value of measuring customer satisfaction (Reichheld, 2003). The idea is to provide a score based on subtracting less satisfied detractors from the highly satisfied promoters and generating a net score. Reichheld further suggests that his score is the best predictor of organizational growth (Reichheld, 2006). To clarify the concept of net promoter, the following definition is provided.

Net promoter is a metric, derived from survey responses to a "likelihood to recommend" question. Respondents who provide a rating of 9 or 10 are classified as "promoters"; respondents who provide a rating of 6 or lower are classified as "detractors." Net promoter is calculated by subtracting the proportion of a firm's detractors from its proportion of promoters (i.e., Net Promoter $=$ promoters - detractors $),($ Keiningham et al, 2007).

In their longitudinal examination of 21 firms, and 15,500 interviews from the Norwegian Customer Satisfaction Barometer, Keiningham, and his co-authors referenced above, debate the superiority of the metric by replicating the analyses used in net promoter research. They also compare Reichheld and colleague's findings with the American Customer Satisfaction Index. 
Ultimately their research failed to replicate Reichheld's claims of clear superiority of his metric versus other measures.

Business executives like the measurement due to its simplicity, and the ease with which they can communicate improvements in the metric to employees. Academics and market researchers argue that by collapsing the 11-point scale, used to collect likelihood to recommend data points, into three components (Promoters, Passives, and Detractors), significant information is lost and statistical variability of the result increases (Business Week, January, 2006).

The important point to consider is that the net promoter metric does not facilitate any understanding of the underlying variables that affect the score, supposedly representative of loyalty. An approach such as the stepwise multiple regression analysis, mentioned above, can provide more information as to the driving forces behind loyalty as a dependent variable.

One may be likely to recommend the company to others because it is a good company for which to work. But, for varied reasons, the employee may not see one's own position as a fit for anymore. Thus, a method is sought that does not assume the link between likelihood to recommend and the propensity to seek a new position outside the organization. 


\subsection{Description of the Dataset - The Specific Situation}

The dataset used to develop the method is comprised of a total of 2,288 online surveys administered in January 2007, through Monster.com, the largest job search engine in the world. The site is primarily used to help those seeking work to find job openings that match their skill set and location requirements. In addition to answering questions about their search behaviors, current company, and supervisor, respondents revealed their job seeking status. Segmenting seekers, non-seekers, and passives, attitudes and behaviors that differentiated the segments were analyzed, particularly as related to their current company, supervisor, quality of work life, and perceptions of opportunity outside their current situation.

\section{Employment Status}

The primary analysis was conducted on only those 1,817 respondents who reported themselves as currently employed full-time (Figure 1.3.1). Moving forward this employed group will be discussed versus the entire dataset. The intent is to reduce bias that may be introduced by those employed part-time, temporarily, or under contract, as other external factors may have substantial effects on their job-seeking behaviors and perceptions of the company, supervisor, quality of work-life, and opportunities.

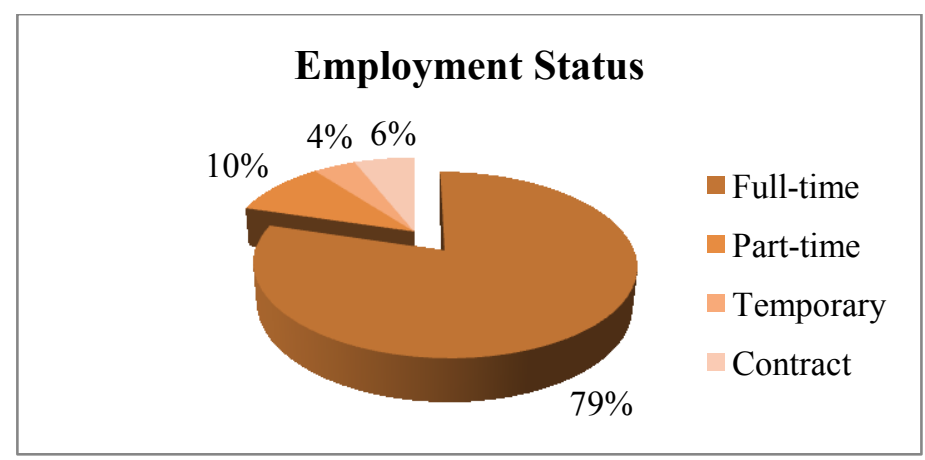

Figure 1.3.1 Employment status 


\section{Job-Seeking Status}

Considering this dataset of respondents that are currently employed full-time, $69 \%$ classified themselves as currently seeking, $26 \%$ passive, and $6 \%$ nonseekers (Figure 1.3.2).

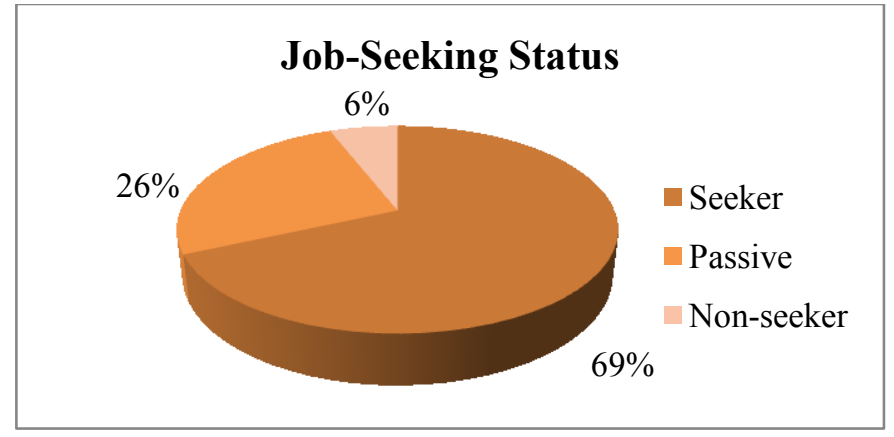

Figure 1.3.2 Job seeking status

Level of Employment

Respondents were asked to reveal their current level of employment. Fortyfour percent labeled themselves as professional/technical, 29\% managerial, 17\% administrative, $7 \%$ executive and 2\% contract/self-employed (Figure 1.3.3).

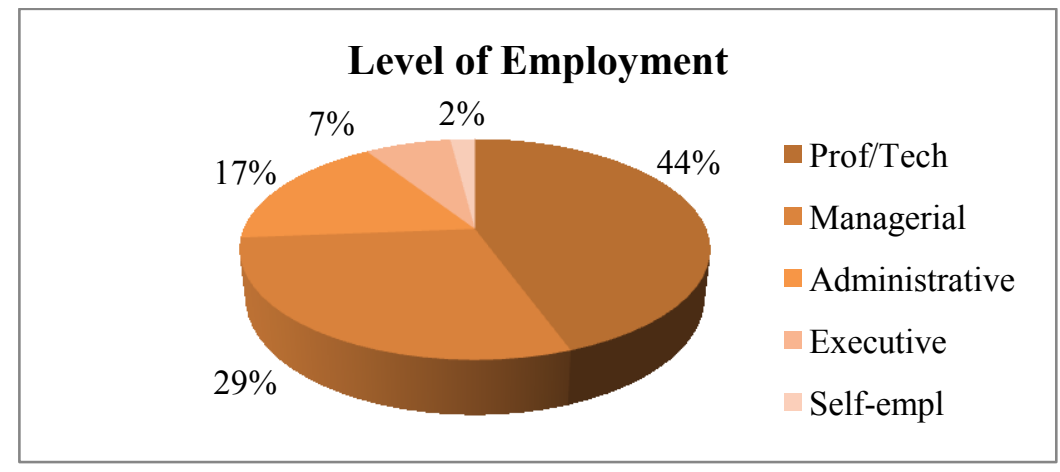

Figure 1.3.3 Level of employment

\section{Most Recent Job Change}

In the discussion of results, significant differences between the subclasses (seeker, passive, non-seeker) are investigated. Considering only the jobseeking status of the employed respondents, distinguishing differences between states is challenging, yet some important highlights are extracted that 
reflect the somewhat "dynamic" state of employees regarding their current positions.

Of those currently employed, when asked how long it had been since their last job change, $50 \%$ reported less than or equal to 2 years (Figure 1.3.4). Of that group, $65 \%$ classified themselves as seekers, and another $27 \%$ as passive, (would leave for right opportunity). Only $8 \%$ were reportedly not seeking a new job. This suggests $92 \%$ of hires within 2 years are in a "dynamic" mode, either seeking or passively seeking. However, the forum (a job search engine) in which the data was collected must be taken into consideration. Of those who had been in their current position 3-5 years, $71 \%$ were seeking, $26 \%$ passive and 3\% non-seeking. The most tenured groups, $26 \%$ in their positions 6 or more years, still reflect $73 \%$ seekers, and $24 \%$ passives.

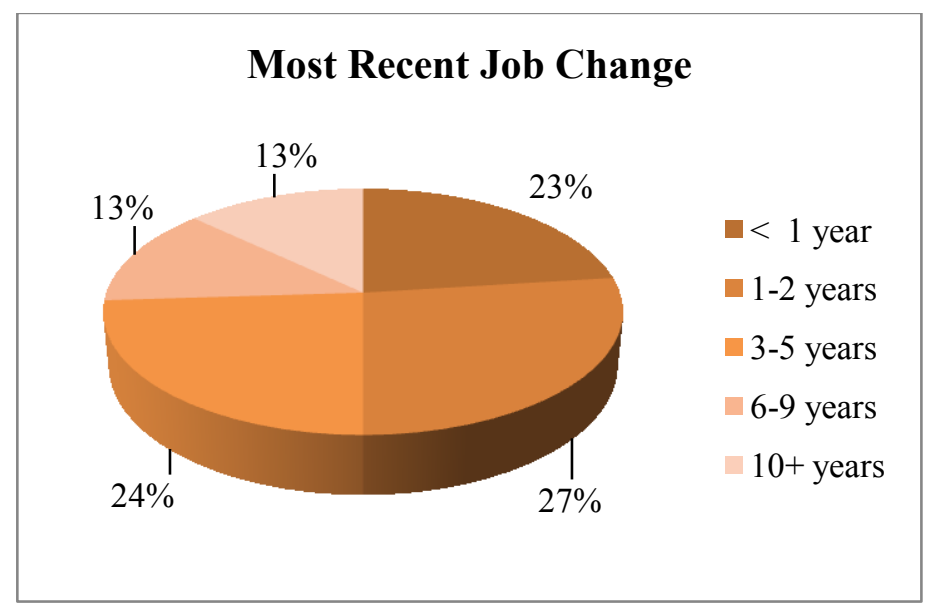

Figure 1.3.4 Most recent job change

Salary

More than half the respondents (55\%) reported their current salary level to be less than $\$ 50,000$ per year. Another $25 \%$ were in the $\$ 51,000$ to $\$ 75,000$ range, $11 \%$ earned from $\$ 76,000$ to $\$ 100,000$, and $7 \%$ were earning more than $\$ 100,000$ annually (Figure 1.3.5). 


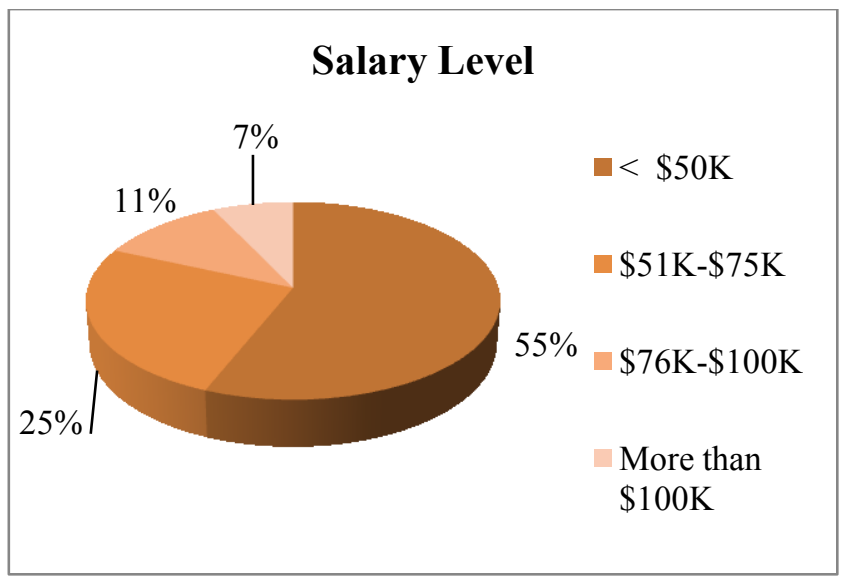

Figure 1.3.5 Salary level

Industries Represented

Over twenty industries were represented in the dataset, with $14 \%$ of the respondents from manufacturing, $12 \%$ professional, scientific, or technical services, $11 \%$ retail trade, 10\% healthcare and social assistance, $9 \%$ from finance, banking, or insurance, $7 \%$ marketing, sales, communications, advertising and public relations, and 5\% in education. Each of the remaining industries was represented by $4 \%$ or less of the respondents (Figure 1.3.6).

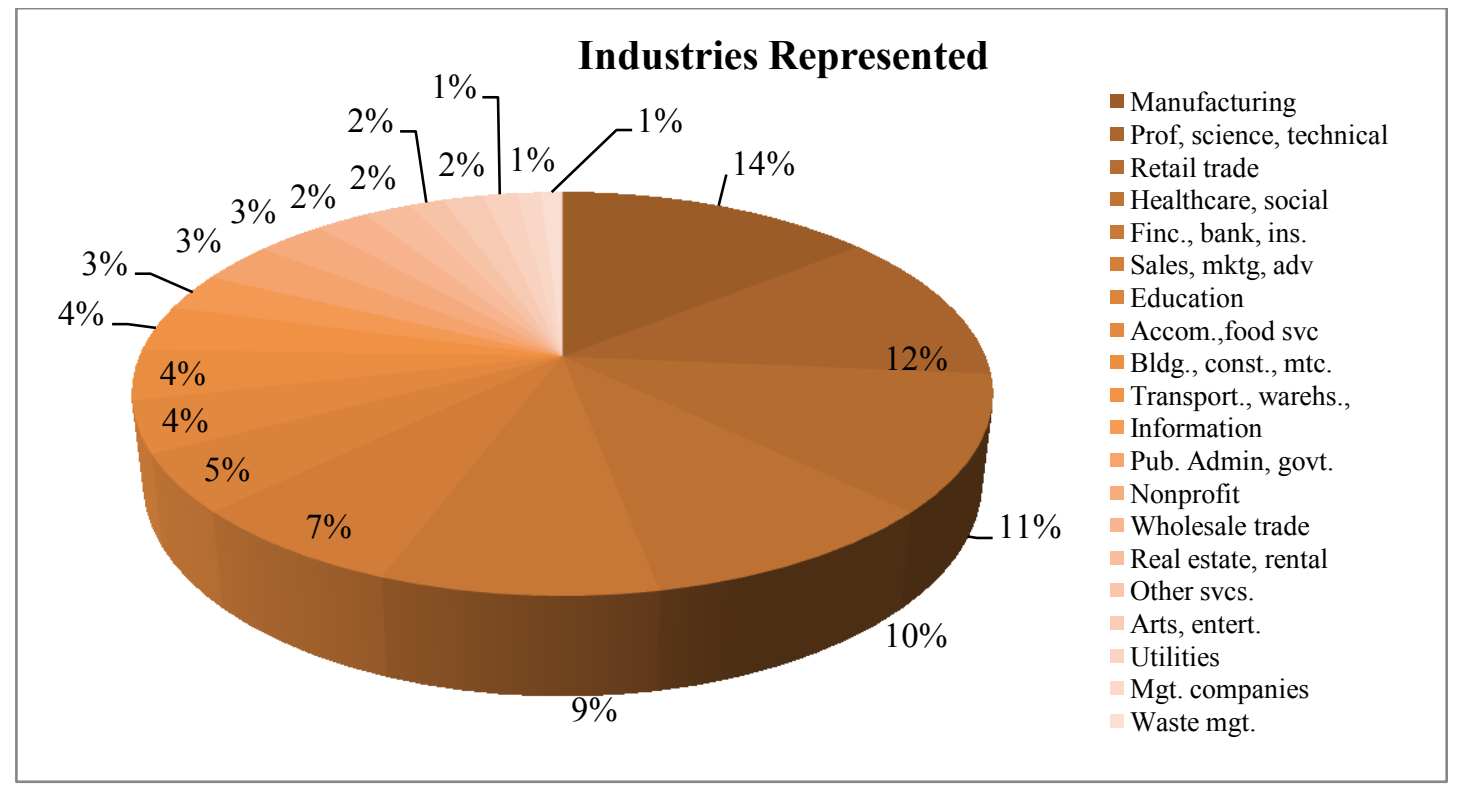

Figure 1.3.6 Industries represented 
Demographics

The gender split was 57\% male, $43 \%$ female (Figure 1.3.7). Thirteen percent are considered Generation Y, 17 to 28 year olds, 33\% Generation X, ages 29 to 41 , and 51\% Baby-Boomers, ages 42 to 60 . Only 3\% were over 61 (Figure 1.3.8).

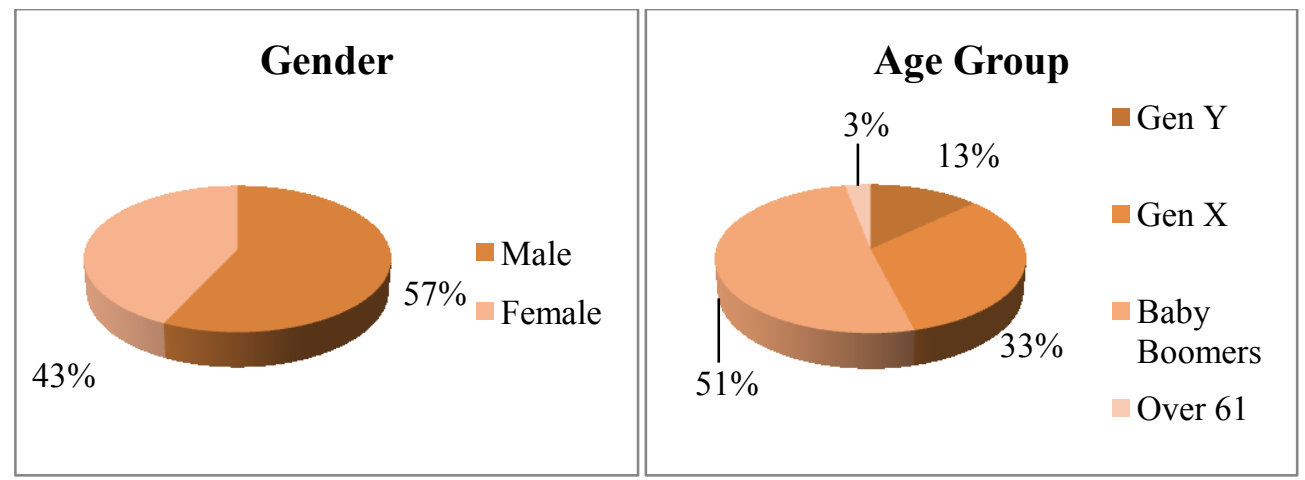

Figure 1.3.7 Respondent gender

Figure 1.3.8 Respondent age group

Respondents were asked to report the region of the United States in which they resided. Twenty-nine percent live in the Northeast, $21 \%$ in the Southeast, $21 \%$ in the Midwest, $18 \%$ in the Southwest, $7 \%$ in the Northwest and $4 \%$ in what is considered the Mountain Region (Figure 1.3.9).

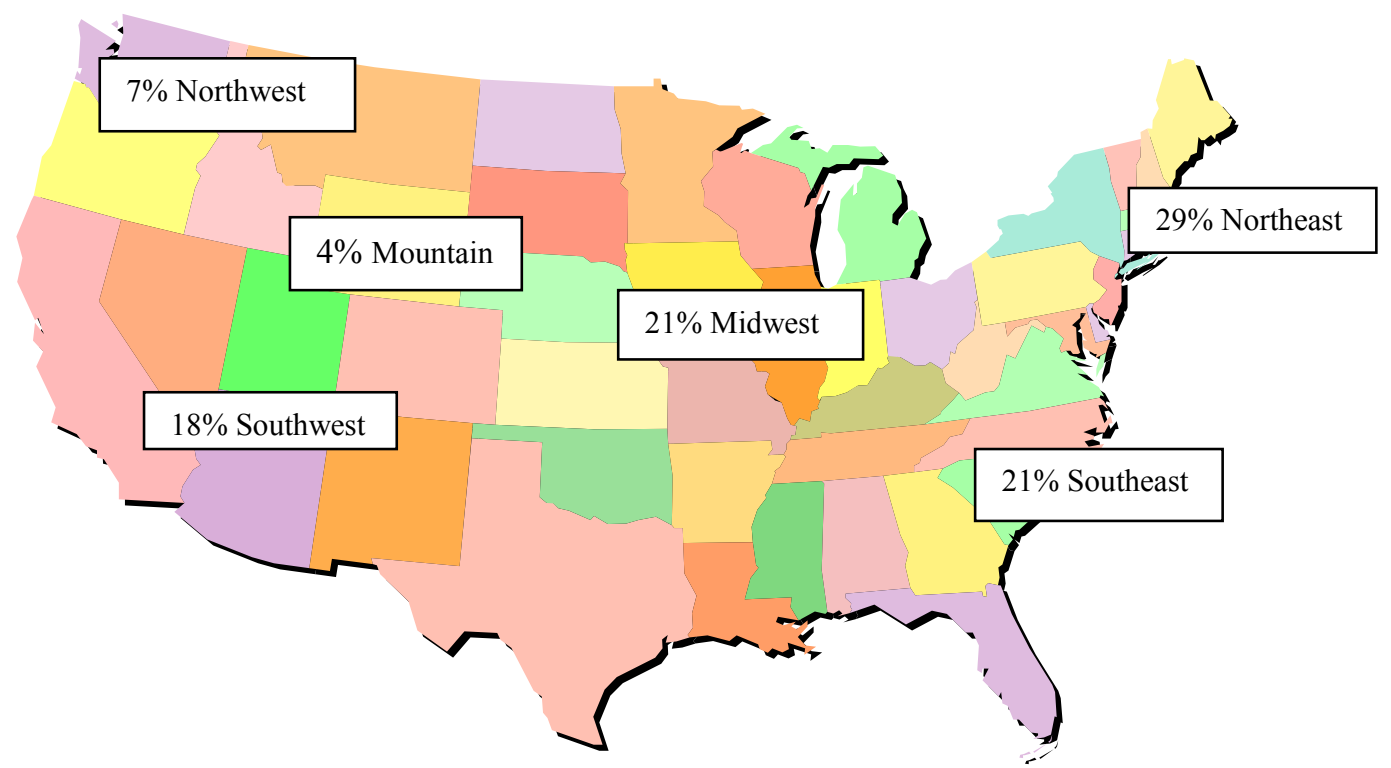

Figure 1.3.9 Respondents' geographic locations within United States 
An ethnicity question was asked, with 74\% reporting that they were white (not of Hispanic origin), 11\% African-American (not of Hispanic origin), 8\% Asian/Pacific Islander, 6\% Hispanic, and 1\% American Indian/Alaskan native (Figure 1.3.10).

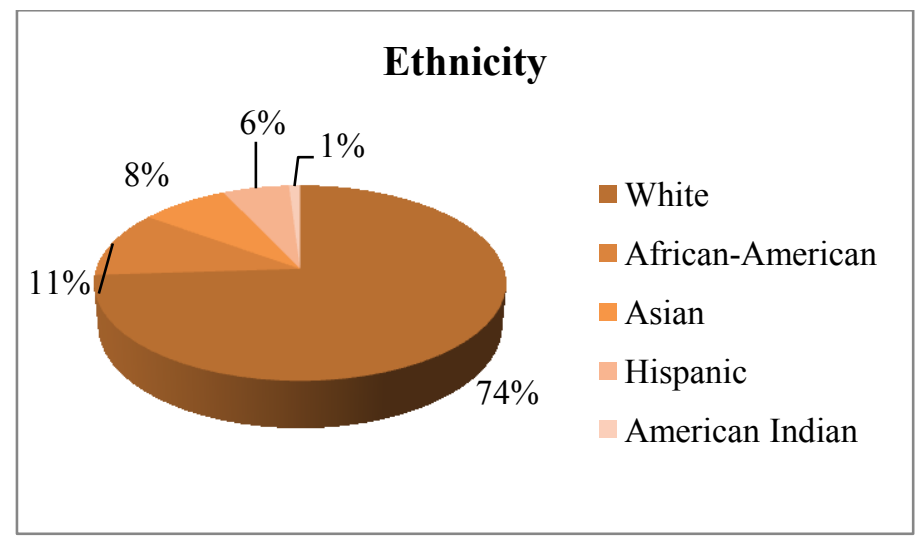

Figure 1.3.10 Respondent ethnicity

Considering education, over a third, (34\%) hold a bachelor's degree, and another $19 \%$ had participated in post-graduate education. Thirteen percent held an Associate's degree (2 year program, typically from community colleges). Another $24 \%$ had attended some amount of college, and $8 \%$ had only graduated high school. One percent had not completed high school (Figure 1.3.11).

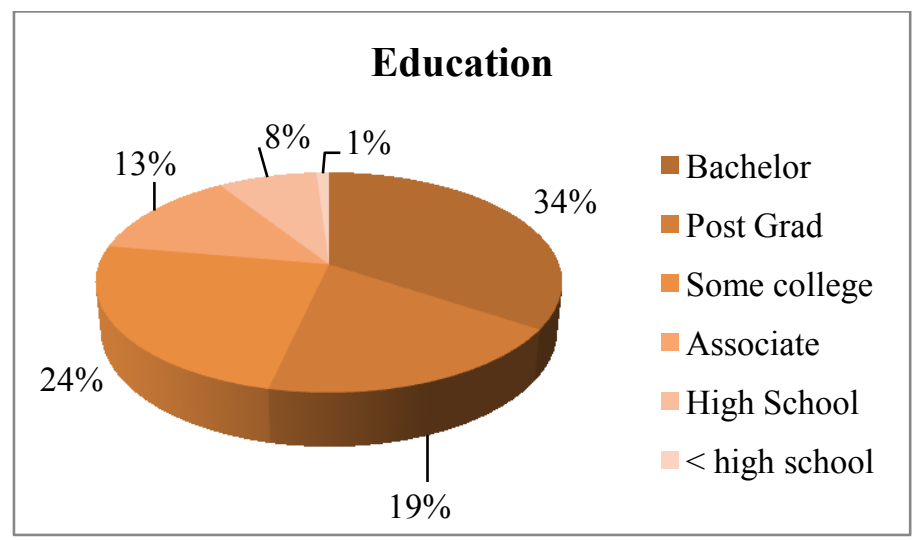

Figure 1.3.11 Respondent education level

To summarize the demographics of this dataset, there is a realistic representation of both genders, more than half of the respondents held a bachelor's degree or higher, and with $90 \%$ having achieved at least some amount of college education. The respondents were primarily non-Hispanic whites (74\%), with a good representation of the varied United States regions, given the population of each. Just slightly more than half are Baby-Boomers (ages 42 to 60), with another 33\% considered Generation X (29-41). 
Areas Investigated

While numerous questions were asked which related to job-seeking behaviors, those that would provide information about the respondent's perceptions of their current workplace and supervisor are analyzed, as well as questions about the extent to which certain external influences would draw them away from their current situation. These related to both the quality of work-life and alternate opportunities. Respondent used a $1=$ low to $10=$ high scale, and following are Table 1.3.1 to Table 1.3.5 listing the questions from which the data was drawn. These tables are presented in the order in which the respondent completed the online questionnaire. Questions about current company included:

\begin{tabular}{l}
$\begin{array}{l}\text { Consider each statement relative to the company you work for and } \\
\text { rate (1=not at all strong, } \mathbf{1 0}=\mathbf{v e r y} \text { strong) how strongly you agree or } \\
\text { disagree }\end{array}$ \\
\hline My company is a good place to work \\
\hline My company provides a valuable product or service \\
\hline My company is honest \\
\hline My company provides a competitive salary \\
\hline My company offers good benefits \\
\hline I think other companies provide better salaries and benefits \\
\hline I would recommend my company to a friend or relative \\
\hline Ifeel proud to work at my company \\
\hline
\end{tabular}

Table 1.3.1 Current company questions

External influence questions:

\begin{tabular}{l} 
If you were to consider a new job, how strongly \\
$(\mathbf{1}=$ not at all strong, $\mathbf{1 0}=\mathbf{v e r y}$ strong) would each of these influencers \\
impact your decision to accept the new position? \\
\hline A higher salary \\
\hline Better benefits \\
\hline Greater advancement opportunities \\
\hline Better skills training \\
\hline A location with a shorter commute \\
\hline A location with better perceived weather \\
\hline A better company \\
\hline The chance to work with a previous supervisor I enjoyed working with \\
\hline The chance to work for a new supervisor \\
\hline Flexible work hours \\
\hline Table 1.3 .2 External influence questions
\end{tabular}


Questions about current supervisor:

\begin{tabular}{l}
$\begin{array}{l}\text { Consider each statement relative to your work supervisor } \\
\text { and rate (10=strongly agree) how strongly you agree or } \\
\text { disagree with it. My supervisor: }\end{array}$ \\
\hline Is likeable \\
\hline Treats me fairly \\
\hline Provides clear directions for work \\
\hline Offers an equitable amount of their attention \\
\hline Sets clear expectation \\
\hline Lets me know what is expected of my work \\
\hline Supports my personal life as much as my work life \\
\hline Supports an equal work/life balance \\
\hline Expects too much from me \\
\hline Is a good mentor \\
\hline Provides me with honest feedback \\
\hline Expects me to do work in off-hours \\
\hline Table 1.3.3 Current supervisor questions \\
\hline
\end{tabular}

A fourth section of company-related questions were asked after the supervisor questions.

\begin{tabular}{|l|}
$\begin{array}{l}\text { Consider each statement below relative to the company you } \\
\text { work for and rate }(\mathbf{1 0}=\text { strongly agree) how strongly you agree } \\
\text { or disagree with it. }\end{array}$ \\
\hline I am fairly compensated for the work I do \\
\hline My company offers higher than average salaries \\
\hline My company provides a competitive package of benefits \\
\hline Table 1.3.4 Additional company questions \\
\hline
\end{tabular}

And, as mentioned earlier, respondents were then asked to report their jobseeking status.

\begin{tabular}{|l|}
\hline Are you currently seeking a new job opportunity? \\
\hline Yes, actively seeking \\
\hline No, staying in my current job for awhile \\
\hline No, but would consider a new job if it were the right opportunity \\
\hline Table 1.3.5 Seeking status question and response categories \\
\hline
\end{tabular}

Consider the first group in the table "seekers", the second group "non-seekers" and the third group "passive seekers", who would consider leaving given the right opportunity. 


\subsection{General Problem}

Next discussed is the general problem of loyalty and its objective measurement as it applies to the job-seeking market. Specifically, is loyalty analytically verifiable in this case?

Consider an employer who wants to understand the stability of his/her organization from a personnel standpoint. If he simply asks each employee if they are seeking, passive, or not seeking, it is highly likely that the answers would overwhelmingly sway to the not seeking side. It is not in an employee's best interest to let his employer know that he is looking for or would consider a new job given the right opportunity. Traditionally, the employer would then move to find a suitable replacement as soon as possible, in expectation of the employee's departure from the organization. Essentially, an employer cannot garner an honest, reliable response from an employee when asked about the status of job-seeking intentions in a direct manner.

The same principles apply in other settings such as business-to-business relationships, and to a certain extent, consumer behavior. If there is a significant perceived risk in allowing one's financial source (employer, client, business-to-business relation, etc.) to know one is considering other options, which could in turn damage or sever the relationship, honest and reliable admission of the potential for transition may be thwarted.

In the job-seeking application discussed here, the goal is to assess loyalty in an alternate manner which provides accurate classification of seeking status. Specifically, the intent is to generate accurate responses that enable classification, without directly asking employees the question, "are you seeking a new job?" or "do you classify yourself as seeking, passive or nonseeking?"

In section 1.2, measures of loyalty were discussed, particularly as ascertained from recommendation likelihood. In section 1.3 the dataset and the variables were discussed. It is appropriate here to elaborate on an existing method which was the catalyst in attempting to uncover an alternate, equally if not more reliable, method to measure loyalty. As mentioned in section 1.2, multiple regression analysis can be employed in an attempt to reveal the drivers of loyalty when there is a measurement for likelihood to recommend.

But, the question remains, is likelihood to recommend truly indicative of loyalty, and more importantly, in our context, is loyalty indicative of jobseeking status? Consider the following opportunity for misrepresentation of loyalty as derived from recommendation likelihood. One might honestly answer that they would not recommend the company to a friend or relative 
simply because none of their friends or relatives may be qualified for the types of positions held at the company. It could be the case that the question is irrelevant to the respondent, and its intent misinterpreted. Low ratings may be the result of an honest admission that the respondent has no opportunity to recommend the company to others. Yet, low ratings would increase variance and the researcher would be investigating the other variables in search of reasoning, when the true reason was simply that the respondent had no application for the variable in question, or in effect, no or low likelihood to recommend.

Consider the question for which there is response data. In our survey, the question was posed among a set of company-related questions, which respondents rated on a 1 to 10 scale of agreement, as follows:

Consider each statement relative to the company you work for and rate ( $1=$ not at all strong, $10=$ very strong), how strongly you agree or disagree with it.

- My company provides a valuable product or service

- My company is honest

- My company provides a competitive salary

- My company offers good benefits

- I think other companies provide better salary and benefits

- I would recommend my company to a friend or relative

- I feel proud to work at my company

Next the results of a regression analysis on the data are discussed which ultimately leads to the variables considered in the factor analysis in Section 2.1 .

Stepwise Regression Analysis

Using the stepwise regression procedure can be insightful, but as some researchers suggest, dangerous due to effects of collinearity, redundancy and suppression (Wuensch, 2006).

The insightful nature of stepwise regression comes into play as potential predictors are investigated that are eventually isolated for use in the factor analysis. In the dataset there were results from 8 questions about the company, 10 regarding external influences on job change, and twelve about the supervisor. These were narrowed down from thirty variables to nineteen which ultimately proved most useful in the factor analysis. Through a series of investigative tests using stepwise regression, nineteen variables were selected 
based on their predictive power as related to likelihood to recommend, as well as company pride and perceptions of honesty. The stepwise regression discussion is limited to that which involves the nineteen variables under investigation.

\section{Sequential Variable Selection Procedures}

During the early 1960s, with the growth of computing capability, several variable selection procedures were developed based on sequential $F$-tests. The idea was to efficiently isolate a subset of the most useful regressor variables when faced with a large number of variables from which to select. Myers (1990) suggests the analyst keep the underlying motivation in mind, which at the time was computational efficiency given that it was prohibitive to gather information from all subset models. Current software packages such as SPSS and SAS offer at least some important results on all subsets. It is known that multicollinearity may cloud the results, and confuse the researcher, yet the varied methods of variable selection remain popular, probably due to their simplicity when applied from a statistical software package. The three primary methods are forward selection, backward elimination and stepwise regression. Each method adds and/or deleted regressor variables from the existing model based on $F$-tests.

The stepwise procedure is actually a modification of the forward selection procedure, so this is discussed first. With forward selection, the initial model contains only a constant term. The first variable for entry into the model is selected by determining the single variable that produces the largest $R^{2}$. Consider this first variable $x_{1}$. Next, a second predictor is selected that produces the largest increase in $R^{2}$ given the presence of $x_{1}$, call this $x_{2}$. This is essential in choosing the regressor with the largest partial $F$ in the sense that the model includes the new variable being pulled into the equation, along with the existing selections.

So, at this second stage identify the largest $F$ :

$$
F=\frac{R\left(x_{2} \mid x_{1}\right)}{s^{2}\left(x_{1}, x_{2}\right)}
$$

Here the denominator, $s^{2}\left(x_{1}, x_{2}\right)$ is the residual mean square for the model involving regressors $x_{1}$ and $x_{2}$. This is typically computed from the incomplete model, in other words, the existing model at that stage. The process continues until the candidate regressor for entry does not meet the 
predetermined criteria for $F_{\mathrm{IN}}$, which in our case is .05 , or all variables are in the equation.

The stepwise method modifies the forward selection procedure by essentially re-evaluating, through a partial $F$-test, all regressors currently in the model upon entry of a new candidate variable. A critical value is preselected (.10 here), for variables to be removed due to multicollinearity. So, a variable may be accepted into the equation initially, and then later removed because it is redundant. The value is that the variable, once accepted, must continue to perform effectively or it is removed. The procedure stops when no additional regressors can be entered based on our critical value $F_{.05}$ for incoming considerations, and $\mathrm{F}_{.10}$ for those to be eliminated.

A final option is backward elimination which begins with all potential regressors and eliminates them one at a time. The first variable removed is the one with the least impact on decreasing $R^{2}$, and thus the smallest partial Fstatistic. The procedure continues until the regressor under consideration for removal results in a partial $F$ which exceeds, in this case, .10.

Chatterjee et al (2000) recommend the backward elimination procedure over forward selection because it allows the researcher to review the full model with all variables considered, even if he selects a more manageable final model. Further, Chatterjee does not recommend the use of variable selection procedures when collinearity exists, yet the backward elimination procedure handles the situation better than does the forward selection procedure (Mantel, 1970).

To check for collinearity, calculate the variance inflation factors (VIFs) or the eigenvalues of the correlation matrix of the predictor variables. The variance inflation factor is defined as

$$
V I F_{j}=\frac{1}{1-?_{j}^{2}} \quad, j=1, \ldots, p
$$

Where $p$ is the number of predictor variables. If there is no linear relationship between the predictor variables (i.e., if they are orthogonal), $R_{j}^{2}$ would be zero and $V I F_{j}$ would be one. Increases in the variance inflation factor suggest that the predictors may not be orthogonal, and collinearity may be present. The value of $V I F_{j}$ also measures the amount by which the variance of the $j$ th regression coefficient is increased due to a linear association between $X_{j}$ and the other predictor variables, relative to the variance expected if $X_{j}$ were not linearly related to them. 
If none of the variance inflation factors are greater than 10 , collinearity is not a problem. If they are, then multicollinearity may cause issues with estimation and interpretation.

Also, the presence of small eigenvalues indicates collinearity. If any are less than 0.01 , the variables are considered collinear. Also look at the condition number, or condition index, which should not be higher than fifteen. The condition number is defined by

$$
k=\sqrt{\frac{\lambda_{\text {-ax }}^{-}}{\lambda_{\text {in }}}}
$$

where $\lambda_{\text {ax }}$ and $\lambda_{-n}$ are the maximum and minimum eigenvalues of the matrix of correlation coefficients (Chatterjee et al, 2000).

Given these criteria, Table 1.4.1 suggests there may be collinearity issues with the data using the stepwise procedure because the condition number is too high for the final variable in the model, the influence of better weather. One option is to simply delete this final variable from the model since it adds only slightly to the explanation of variance, with $R^{2}$ change $=.001$, and the significance of the change in $F$, is .024 . The VIFs are acceptable, with none approaching 10, not even the influence of better weather. However, the eigenvalue for this variable, which should not be less than .01 , is .018 , alerting the researcher of potential collinearity.

Overall, the regression model built with the stepwise procedure is a good one, with nearly $77 \%$ variance explained and strong values for the $t$ statistic which typically exceed 2.0 if significant. However, sacrificed is all the information obtained from the other twelve variables. Subsequent analyses suggest these others add valuable insight, particularly when working to differentiate seeking status. More specifically, the regression eliminates most of the external influence variables, with the exception of the influence of a better company, and the somewhat questionable variable, the influence of better weather in a different location. Further, the supervisor variables do not survive the stepwise procedure, with the exception of fair treatment from the supervisor. Lastly, the respondent's perception of their company providing valuable products and services is lost which intuitively would seem to be a good measure of loyalty. It should be noted that the application of stepwise regression is most useful in suggesting areas of operational focus, which in this case would be developing company pride, ensuring salaries are competitive, good benefits are provided and that the company is at least perceived as honest. This loss of information is precisely the reason to move 
beyond stepwise regression analysis to solve the general problem of measuring loyalty in a more scientific manner.

\begin{tabular}{|llllll|}
$\begin{array}{l}\text { Variables in the } \\
\text { loyalty regression model }\end{array}$ & $\mathbf{R}^{\mathbf{2}}$ & T statistic & VIF & Eigenvalue & $\begin{array}{l}\text { Condition } \\
\text { number }\end{array}$ \\
$\begin{array}{l}\text { Proud to work at } \\
\text { company }\end{array}$ & .725 & 29.88 & 2.80 & .27 & 5.18 \\
\hline Competitive salary & .751 & 7.44 & 1.84 & .133 & 7.39 \\
\hline Good benefits & .761 & 8.20 & 1.70 & .123 & 7.69 \\
\hline Honest & .766 & 5.01 & 2.38 & .072 & 10.05 \\
\hline Supv. treats me fairly & .766 & 2.29 & 1.36 & .068 & 10.35 \\
\hline Infl. better company & .767 & -2.95 & 1.17 & .039 & 13.60 \\
\hline Infl. better weather & .768 & 2.26 & 1.15 & .018 & 20.16 \\
\hline
\end{tabular}

Table 1.4.1 Variables in the loyalty regression model 


\subsection{Problem of Classification by Subclass}

In this section the significant differences are discussed regarding each of the three groups including job seekers, passives that would take a new position given the right opportunity, and non-seekers who are content to stay in their current position for a while. Understanding the differences between each of the seeking statuses can help us make inferences about the most prominent catalysts for transition from state to state. Further, if one wants to be able to identify an individual as a member of one of the groups, there is a need to understand the profile and characteristics of each group, and what makes them differ from one another.

In a general sense, to test for significant differences between any two of the seeking status groups of respondent types in the dataset (i.e., passives vs. seekers, seekers vs. non-seekers, and non-seekers vs. passives), consider the mean scores for the variables measured, particular to each group, and the differences between these individual group means.

Keep in mind that one group is compared to another, one at a time, as independent samples, and therefore the $t$-test is used, which Lowry suggested "is probably the most widely used statistical test of all time, and certainly the most widely known. It is simple, straightforward, easy to use, and adaptable to a broad range of situations. No statistical toolbox should ever be without it." (Lowry 2009). Another practical reason for using a commonly known test such as the $t$-test is that the aim is to create a useful tool for applications in business settings, and most users will have had some exposure to the $t$-test in business school, and would be more likely to understand the methodology implemented here.

Consider the null and alternative hypotheses for each of the three examinations. As a matter of notation, seekers are coded as group 1, passives are in group 2, and non-seekers in group 3, indicative of increasing preference toward a non-seeking employee.

Research question 1: Are there significant differences between non-seekers (group 3) and seekers (group 1)?

Research question 2: Are there significant differences between non-seekers (group3) and passives (group 2)?

Research question 3: Are there significant differences between passives (group 2) and seekers (group 1)? 


$$
\begin{array}{lll}
H 1_{0}: \mu=\imath & H 2_{0}: \mu=\imath & H 3_{0}: \mu_{-}=\imath \\
H 1_{a}: \mu \neq \imath & H 2_{a}: \mu \neq \imath & H 3_{a}: \mu \neq \imath
\end{array}
$$

In each of the above hypotheses statements, the research aims to support the alternative hypotheses, that the mean scores of the variables, examined for the two independent samples (or groups of job-seeking types), differ significantly. The hope is to reject the null hypothesis for each of the research questions, which states that any difference found between the means of the two samples should not significantly differ from zero.

Generating Difference Analysis

Consider one group compared to one other group (e.g., seekers vs. passives, passives vs. non-seekers, seekers vs. passives). Use an independent samples ttest procedure with a $95 \%$ confidence interval, and exclude cases analysis by analysis, meaning that each t-test uses all cases that have valid data for the tested variables, and sample sizes may vary from test to test.

The independent samples t-test procedure in SPSS produces two tests of difference between the two groups under observation. One test assumes variances of the two groups are equal, tested by Levene's test for homogeneity of variances (Levene, 1960). If Levene's test statistics is greater than 0.10, assume groups have equal variances and ignore the second test which does not assume equality of variances. If Levene's statistic is less than 0.10 , use the $t$ statistic from this second test in which equal variances are not assumed.

Another reason to use Levene's test is because it is thought to be less sensitive to departures from normality, than say the Bartlett test (NIST SEMATECH, 2009). As will be detailed in subsequent sections, non-normality is present, thus a conservative approach was taken and Levene's test for equality of variances applied.

In this analysis, in virtually all cases, the $t$-statistic was used for which equal variances were not assumed. The exception was the variable which measured the influence of the chance to work with a new supervisor, in which case the Levene's statistic was greater than 0.10 and homogeneity of variances was assumed. Levene's test statistic is defined as follows:

$$
W=\left(\frac{N-k}{k-1}\right)^{\frac{\sum_{i=1}^{k} N_{i}\left(\bar{Z}_{i .}-Z . .\right)^{2}}{\sum_{i=1}^{k} \sum_{j=1}^{N_{i}}\left(Z_{i j}-\bar{Z}_{i .}\right)^{2}}}
$$


where

$\mathrm{W}$ is the test statistic

$k$ is the number of groups which contain the samples

$N$ is the total samples

$N_{i}$ is the number of samples in the $\mathrm{i}^{\text {th }}$ group

and define

$Y_{i j}$ as the value of the $\mathrm{j}^{\text {th }}$ sample from the $\mathrm{i}^{\text {th }}$ group

Also,

$Z_{i j}=Y_{i j}-{ }_{i}{ }_{i} \mid, \quad \bar{Y}_{i}$ is the mean of the $\mathrm{i}^{\text {th }}$ group

$Z . .=\frac{1}{N} \sum_{i=1}^{k} \sum_{j=1}^{N_{i}} Z_{i j} \quad$ is the overall mean of all the $Z_{i j}$

$Z_{i .}=\frac{1}{N_{i}} \sum_{j=1}^{N_{i}} Z_{i j} \quad$ is the mean of the $Z_{i j}$ for group $i$.

Based on results from the Levene test, reject the null hypothesis that the variances are equal if

$W>F_{\left(a, k_{-}, N_{-}\right)}$, where $F_{\left(a, k_{-}, N_{-}\right)}$is the upper critical value of the $\mathrm{F}$ distribution with $k-1$ and $N-k$ degrees of freedom at significance level $\alpha$.

Identifying Significant Differences Between Subclasses

Statistically significant differences between the subclasses (seeker, passive and non-seeker) were identified on virtually all the key variables examined. Figure 1.5.1 provides a summary of the variables that evidence significant differences between subclasses. The bulleted list is in descending order of $t$ values. Following the summary figure, the differences are discussed thoroughly. 


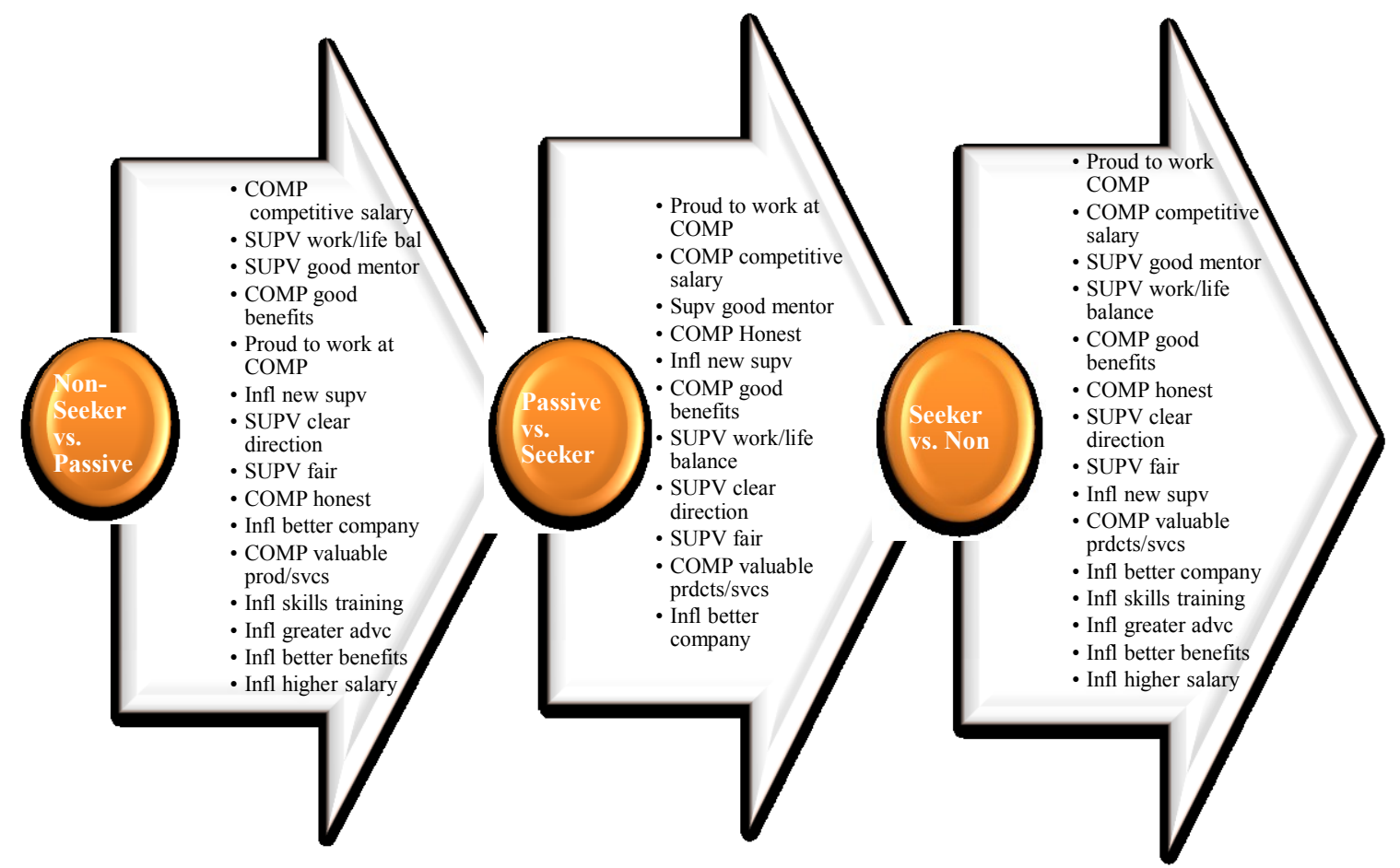

Figure 1.5.1 Variables listed inside arrows evidenced significant differences between groups listed inside oval

Reviewing the most significant differences between an active seeker and one who is still in the passive state, (second arrow), note that the most substantial differences concern the company (pride and salary). The passive is significantly more proud to work for their company, which they perceive as significantly more honest, compared to the seeker. Further, passives are more satisfied with the competitiveness of their salary provided by the company. In fact, the most substantial differences in all states are related to the company variables, more specifically, pride in the company.

This work now transitions to a thorough review of the significant differences between each group. The discussion begins with non-seekers versus seekers, as these are the most diverse groups. The more subtle but significant differences between non-seekers and passives, and passives versus seekers are then investigated.

\section{Non-seekers Compared to Seekers}

Significance tests for mean differences indicate, as expected, substantial differences between those content to stay at their current company, versus those who are actively seeking. The largest differences are in regard to company pride which non-seekers rate $64 \%$ higher than seekers. Specifically, non-seekers rate their pride at 8.48 versus seekers at 5.17. Table 1.5.2 offers a comparative list of ratings for non-seekers versus seekers. The mean scores for 
each group are provided for each of the variables which evidenced a statistically significant difference. The table also provides a simple percentage difference calculated as follows:

$$
\frac{\bar{x}_{\text {non-eeker }}-\bar{i}_{\text {see } \mathrm{ker}}}{\bar{x}_{\text {see ker }}}
$$

The percentage difference helps one understand the magnitude of the variation between the groups, which is subsequently supported by the t-statistic, reported in the next column, followed by degrees of freedom for the test, the p-value of two-tailed significance, mean difference and standard error of the difference. Subsequent tables of this type can be read in the same manner for easy reference to the prominent differences between groups evaluated.

Non-seekers are nearly $56 \%$ more satisfied with the competitiveness of the salaries at their current company, and rate their supervisor significantly higher as a mentor, $54 \%$ higher, and in supporting work-life balance, $47 \%$ higher.

Non-seekers are $44 \%$ more content with benefits provided by their company, and are significantly more likely to believe that their company is honest. Nonseekers rate company honesty nearly $42 \%$ higher, at 8.49 versus seekers at 6.0 .

The next most substantial differences concern the supervisor again. Seekers rate their supervisor significantly lower $(36 \%$ lower) on providing clear direction than do non-seekers. Further, they tend not to feel that the supervisor treats them fairly, rating their supervisors at 6.42 on this variable, versus nonseekers who rated their supervisors at $8.59,34 \%$ higher.

It follows then that seekers are significantly more influenced by the potential opportunity to work with a new supervisor; nearly $30 \%$ more influenced.

Non-seekers rated their agreement that their company provides valuable products and services at 9.07 versus the seeker rating of 7.44 , nearly $22 \%$ higher. This helps explain why seekers are significantly more influenced $(15 \%)$ by the opportunity to work for a better company.

At this point the external influences become less differentiable between nonseekers and seekers. While the groups do still evidence significant differences on the influences of better skills training, greater opportunity for advancement, better benefits, and potentially higher salary, the gaps are not quite so drastic. Figure 1.5.2 provides a comparison of the means of the variables on which significant differences between non-seekers and seekers were found. Table 
1.5.1 offers further clarification of the percentage difference and the t-test statistics to support these claims.

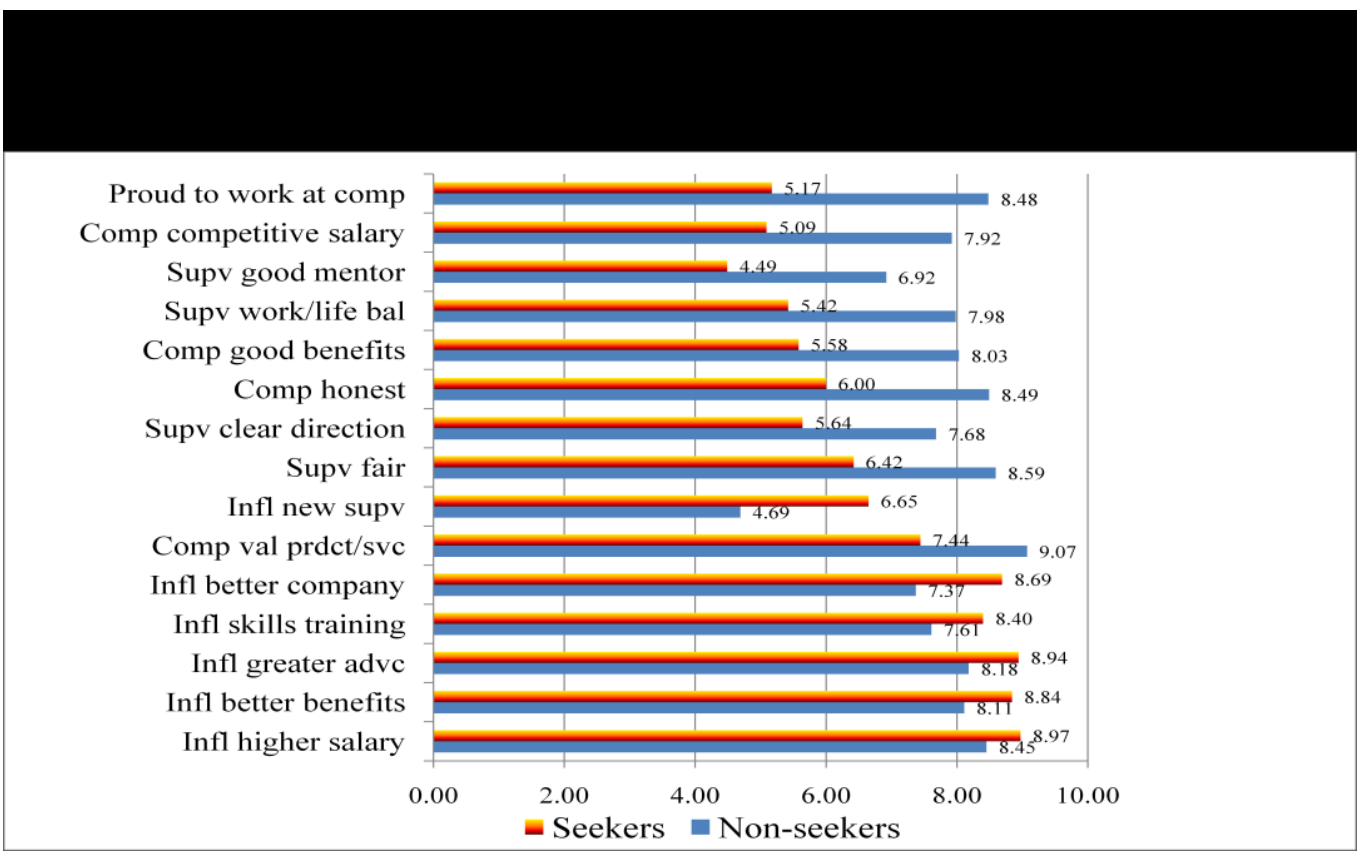

Figure 1.5.2 Non-seeker vs. seeker - significant differences between group means

\begin{tabular}{|lllllllll|} 
& $\begin{array}{l}\text { Non- } \\
\text { seekers }\end{array}$ & Seekers & $\begin{array}{l}\text { Percent } \\
\text { difference }\end{array}$ & T stat & $\begin{array}{l}\text { Degrees } \\
\text { of } \\
\text { freedom }\end{array}$ & $\begin{array}{l}\text { Sig. 2 } \\
\text { tailed }\end{array}$ & $\begin{array}{l}\text { Mean } \\
\text { diff. }\end{array}$ & $\begin{array}{l}\text { Std. } \\
\text { error } \\
\text { diff. }\end{array}$ \\
\hline $\begin{array}{l}\text { Proud to work } \\
\text { comp }\end{array}$ & 8.48 & 5.17 & $64.02 \%$ & -17.24 & 149 & .000 & -3.31 & .192 \\
\hline $\begin{array}{l}\text { Comp } \\
\text { competitive salary }\end{array}$ & 7.92 & 5.09 & $55.60 \%$ & -13.3 & 134 & .000 & -2.82 & .212 \\
\hline $\begin{array}{l}\text { Supv good } \\
\text { mentor }\end{array}$ & 6.92 & 4.49 & $54.12 \%$ & -9.20 & 128 & .000 & -2.43 & .264 \\
\hline $\begin{array}{l}\text { Supv work/life } \\
\text { bal }\end{array}$ & 7.98 & 5.42 & $47.23 \%$ & -11.27 & 140 & .000 & -2.56 & .227 \\
\hline $\begin{array}{l}\text { Comp good } \\
\text { benefits }\end{array}$ & 8.03 & 5.58 & $43.91 \%$ & -11.12 & 138 & .000 & -2.44 & .220 \\
\hline Comp honest & 8.49 & 6.00 & $41.50 \%$ & -12.83 & 147 & .000 & -2.45 & .194 \\
\hline $\begin{array}{l}\text { Supv clear } \\
\text { direction }\end{array}$ & 7.68 & 5.64 & $36.17 \%$ & -8.72 & 133 & .000 & -2.03 & .233 \\
\hline Supv fair & 8.59 & 6.42 & $33.80 \%$ & -11.99 & 156 & .000 & -2.16 & .181 \\
\hline Infl new supv & 4.69 & 6.65 & $-29.47 \%$ & -7.39 & 134 & .000 & 1.95 & .264 \\
\hline $\begin{array}{l}\text { Comp val } \\
\text { prdct/svc }\end{array}$ & 9.07 & 7.44 & $21.91 \%$ & -11.20 & 156 & .000 & -1.62 & .145 \\
\hline $\begin{array}{l}\text { Infl better } \\
\text { company }\end{array}$ & 7.37 & 8.69 & $-15.19 \%$ & 5.196 & 109 & .000 & 1.32 & .254 \\
\hline Infl skills training & 7.61 & 8.40 & $-9.40 \%$ & 3.433 & 112 & .001 & .79 & .230 \\
\hline Infl greater advc & 8.18 & 8.94 & $-8.50 \%$ & 3.327 & 110 & .001 & .75 & .227 \\
\hline $\begin{array}{l}\text { Infl better } \\
\text { benefits }\end{array}$ & 8.11 & 8.84 & $-8.26 \%$ & 3.260 & 110 & .001 & .73 & .224 \\
\hline Infl higher salary & 8.45 & 8.97 & $-5.80 \%$ & 2.632 & 112 & .010 & .52 & .198 \\
\hline
\end{tabular}

Table 1.5.1 Significant differences between the means of non-seekers vs. seekers 
Non-seekers Compared to Passives

It is essentially simpler to state the three variables on which non-seekers and passives do not differ significantly. These are the external influences of shorter commute, better weather, and the chance to work with a previous supervisor. Aside from these three variables, non-seekers and passives differed significantly on all other variables, with non-seekers rating their current company and supervisor significantly higher in most areas, and external influences significantly lower.

Non-seekers are $21 \%$ more likely to be satisfied with salary at their current company, rating this variable at 7.92 , compared to passives who rated it 6.55 .

Interestingly, the largest difference quickly transitions, and to virtually the same extent, to the supervisor's support of work/life balance (7.98 nonseekers, 6.60 passives). Passives rate their supervisor as a good mentor significantly lower than the non-seekers (5.75 versus 6.92 respectively).

The extent of the differences then switch back to company related variables with good benefits garnering significantly greater satisfaction from passives versus non-seekers, nearly $18 \%$ greater. Next, it is company pride that separates passives from non-seekers who rate their sense of company pride $16 \%$ higher than passives.

The subtle yet significant differences between non-seekers and passives continue with the supervisor variables including the influence of the potential to work with a new supervisor, rated as nearly $15 \%$ more influential for passives (5.51 versus 4.69 respectively) compared to their non-seeking counterparts. On the other hand, non-seekers are significantly, 14\%, more satisfied with the clarity of direction they receive from their supervisor, and $12 \%$ happier with the supervisor's fair treatment.

Company honesty plays into the differences next, rated $11 \%$ higher by nonseekers versus passives. In addition to the aforementioned differences between non-seekers and passives on other company related variables (competitive salary, good benefits, company pride, and honesty), passives are significantly more influenced $(9 \%)$ by the potential opportunity to work for what they perceive to be a better company. Passives rate the value of their company's products and services significantly lower (by $8 \%$ ) than do non-seekers.

The differences grow more subtle as the two groups are evaluated on the remaining external influences including skills training, greater opportunity for 
advancement, better benefits and higher salary. It is interesting to note that these influences are typically rated greater than 8 on the 10 point scale by both non-seekers and passives. The groups are different, but again, the variation is subtle and may not be identified initially. Figure 1.5.3 depicts the mean scores for all the significant differences between these two groups, and Table 1.5.2 offers further clarification.

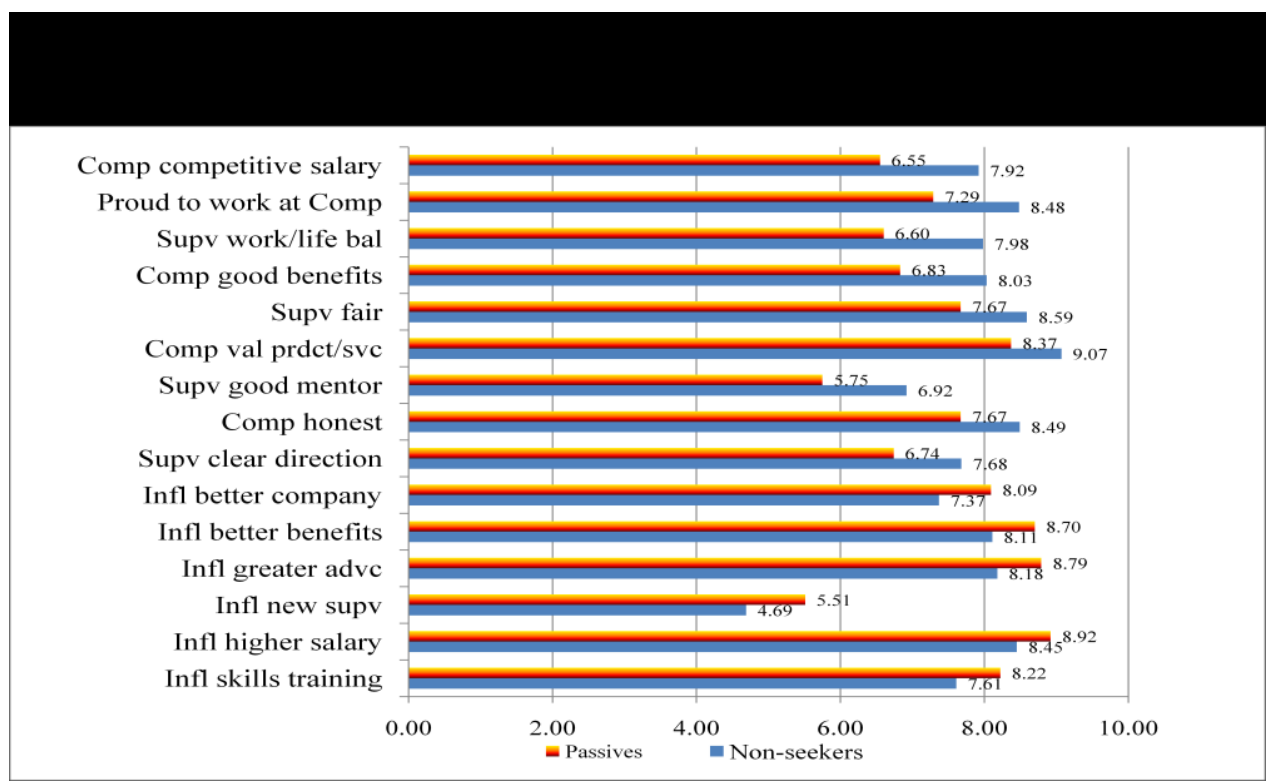

Figure 1.5.3 Non-seekers vs. passives - significant differences

\begin{tabular}{|lllllllll|} 
& $\begin{array}{l}\text { Non- } \\
\text { seekers }\end{array}$ & Passives & $\begin{array}{l}\text { Percent } \\
\text { difference }\end{array}$ & T Stat & $\begin{array}{l}\text { Degrees } \\
\text { freedom }\end{array}$ & $\begin{array}{l}\text { Sig. 2 } \\
\text { tailed }\end{array}$ & $\begin{array}{l}\text { Mean } \\
\text { diff }\end{array}$ & $\begin{array}{l}\text { Std } \\
\text { error } \\
\text { diff }\end{array}$ \\
\hline $\begin{array}{l}\text { Comp competitive } \\
\text { salary }\end{array}$ & 7.92 & 6.55 & $20.92 \%$ & -6.13 & 164 & .000 & -1.37 & .224 \\
\hline Supv work/life bal & 7.98 & 6.60 & $20.91 \%$ & -5.58 & 189 & .000 & -1.37 & .247 \\
\hline Supv good mentor & 6.92 & 5.75 & $20.35 \%$ & -4.15 & 166 & .000 & -1.17 & .283 \\
\hline $\begin{array}{l}\text { Comp good } \\
\text { benefits }\end{array}$ & 8.03 & 6.83 & $17.57 \%$ & -5.12 & 171 & .000 & -1.19 & .233 \\
\hline $\begin{array}{l}\text { Proud to work at } \\
\text { Comp }\end{array}$ & 8.48 & 7.29 & $16.32 \%$ & -5.85 & 184 & .000 & -1.1 & .204 \\
\hline Infl new supv & 4.69 & 5.51 & $-14.88 \%$ & 2.87 & 146 & .004 & .81 & .283 \\
\hline $\begin{array}{l}\text { Supv clear } \\
\text { direction }\end{array}$ & 7.68 & 6.74 & $13.95 \%$ & -3.81 & 164 & .000 & -.94 & .247 \\
\hline Supv fair & 8.59 & 7.67 & $11.99 \%$ & -4.81 & 191 & .000 & -.92 & .192 \\
\hline Comp honest & 8.49 & 7.67 & $10.69 \%$ & -4.04 & 174 & .000 & -.82 & .204 \\
\hline $\begin{array}{l}\text { Infl better } \\
\text { company }\end{array}$ & 7.37 & 8.09 & $-8.90 \%$ & 2.72 & 130 & .007 & .72 & .265 \\
\hline Comp val prdct/svc & 9.07 & 8.37 & $8.36 \%$ & -4.51 & 193 & .000 & -.69 & .154 \\
\hline Infl skills training & 7.61 & 8.22 & $-7.42 \%$ & 2.53 & 132 & .012 & .60 & .240 \\
\hline Infl greater advc & 8.18 & 8.79 & $-6.94 \%$ & 2.56 & 126 & .012 & .60 & .234 \\
\hline Infl better benefits & 8.11 & 8.70 & $-6.78 \%$ & 2.59 & 124 & .011 & .59 & .231 \\
\hline Infl higher salary & 8.45 & 8.92 & $-5.27 \%$ & 2.30 & 126 & .023 & .46 & .203 \\
\hline
\end{tabular}

Table 1.5.2 Significant differences between the means of non-seekers vs. passives 


\section{Passives vs. Seekers}

Next investigated are the differences between passives who would consider leaving the company for a new job, given the right opportunity versus those who are actively seeking (Figure 1.5.4). Passives rate their company and supervisor significantly higher than seekers, but there are no significant differences between passives and seekers on the external influences. This is with the exception of the influence of a new supervisor or better company, where in both situations the passives rate these influences significantly lower than seekers.

More specifically, nearly a $30 \%$ difference is found between passives and seekers on company pride, with passives rating the variable at 7.29 versus seekers at 5.17. Recall, more than double this difference was seen between seekers and non-seekers (5.17 vs. 8.48). Company pride accounted for $72.5 \%$ of the variance in likelihood to recommend the company (see section 1.4).

Consider the strong and interesting relationship between company pride and perceptions of company honesty. Considering the whole dataset of full time employed respondents, the correlation between company pride and honesty is $r=74$, significant at $p<001$. Given each subclass, the correlation for seekers is $r=.709$, passives $r=.690$, and non-seekers at $r=.715$, all significant at $p<001$. This sense of pride may be particularly influential in differentiating seekers from both passives and non-seekers. A 16\% difference between passives and non-seekers is evident on this variable. The general indication is that company pride may serve as a catalyst to loyalty erosion and ultimately, to a transition from state to state.

A hearty significant difference between the two subclasses is found regarding agreement that their company provides a competitive salary. Seekers rated the variables at 5.09 and passives at 6.55 , just over $22 \%$ higher. Recall that perceptions of being employed by a company that offers competitive salary was the largest difference between passives and non-seekers, indicating another potential catalyst to transition.

Note in Table 1.5.3 the consistent shifting between company and supervisor related variables, perhaps indicating the careful balance required to prevent a transition from passive to actively seeking. The next most prominent variation between passives and seekers is with regard to the supervisor being a good mentor which passives rated nearly $22 \%$ higher.

Company honesty was also rated nearly $22 \%$ higher by passives than seekers. Seekers rated the influence of the potential opportunity to work with a new 
supervisor significantly higher than passives (6.65 vs. 5.51), a $21 \%$ difference. Recall the difference between passives and non-seeker which was $15 \%$, with non-seekers significantly less influenced by the opportunity to work with a new supervisor. Jump from non-seekers to seekers, and the difference is substantial at nearly $30 \%(29.47 \%)$.

Seekers are significantly less satisfied with the benefits offered by their current company rating the variable at 5.58 whereas passives rated this at 6.83 , over $18 \%$ higher.

Support of work/life balance, providing clear direction, and fairness, are all rated significantly higher by passives than seekers. Passives rate their perception of the value of the company's products and services significantly higher than seekers, $11 \%$ higher.

Lastly the influence of the potential opportunity to work for a better company is more attractive to those actively seeking versus the passives $(7.42 \%$ higher for passives than seekers), but both groups rate the influence fairly high on the 1 to 10 scale, with seekers at 8.69 , passives at 8.09 , and recall non-seekers rated this external influence at 7.37.

Following are Figure 1.5.4 and Table 1.5.3 to clarify differentiation of passives from seekers.

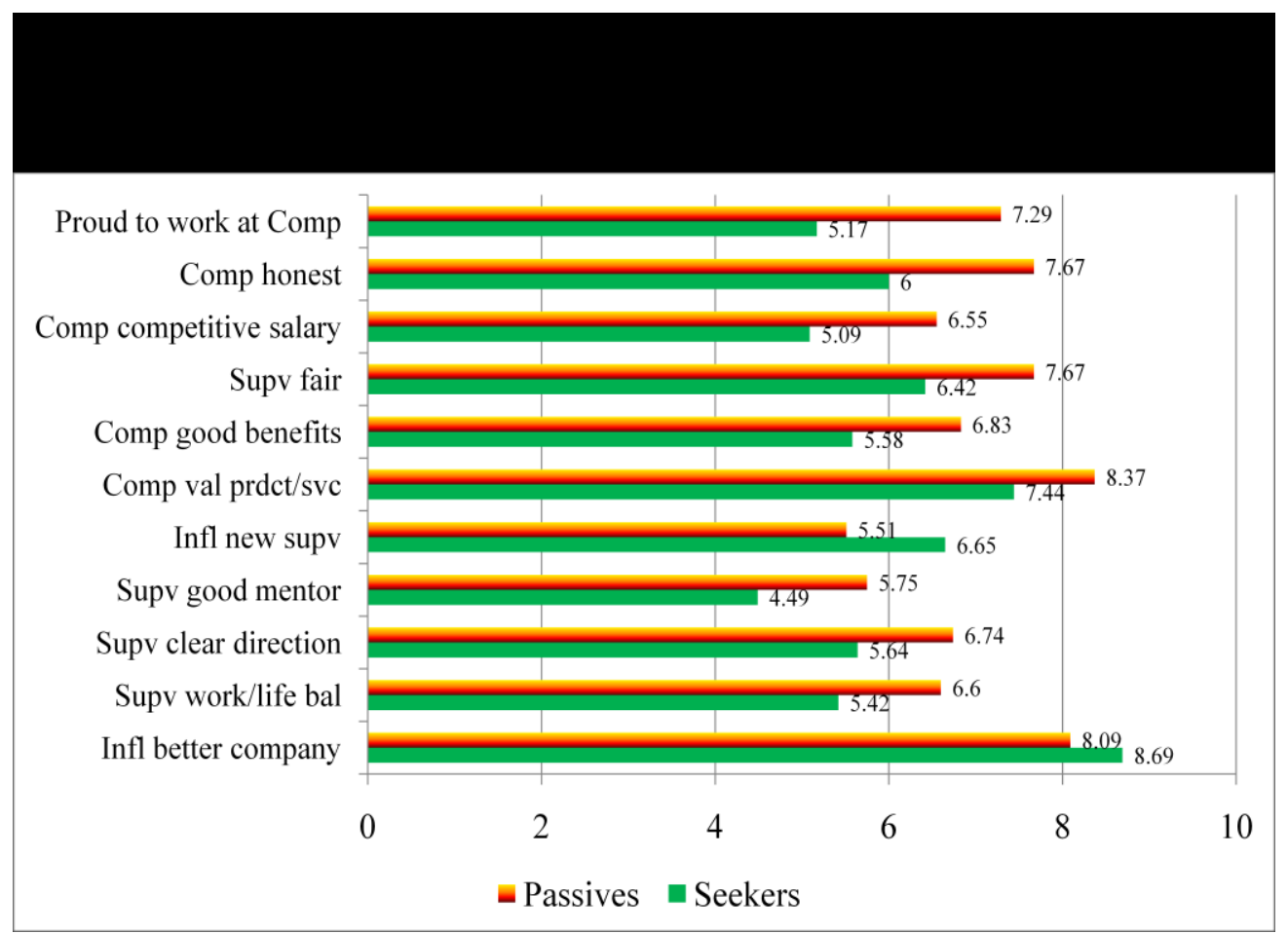

Figure 1.5.4 Passives vs. seekers - significant differences 


\begin{tabular}{|c|c|c|c|c|c|c|c|c|}
\hline & Seekers & Passives & $\begin{array}{l}\text { Percent } \\
\text { difference }\end{array}$ & T Stat & $\begin{array}{l}\text { Degrees } \\
\text { of } \\
\text { freedom }\end{array}$ & $\begin{array}{l}\text { p- } \\
\text { value }\end{array}$ & $\begin{array}{l}\text { Mean } \\
\text { diff }\end{array}$ & $\begin{array}{l}\text { Std. } \\
\text { error } \\
\text { diff }\end{array}$ \\
\hline $\begin{array}{l}\text { Proud to work at } \\
\text { Comp }\end{array}$ & 5.17 & 7.29 & $-29.08 \%$ & -16.130 & 1022 & .000 & -2.12 & .132 \\
\hline $\begin{array}{l}\text { Comp } \\
\text { competitive } \\
\text { salary }\end{array}$ & 5.09 & 6.55 & $-22.29 \%$ & -11.149 & 978 & .000 & -1.45 & .130 \\
\hline $\begin{array}{l}\text { Supv good } \\
\text { mentor }\end{array}$ & 4.49 & 5.75 & $-21.91 \%$ & -7.928 & 875 & .000 & -1.25 & .158 \\
\hline Comp honest & 6.00 & 7.67 & $-21.77 \%$ & -12.84 & 1067 & .000 & -1.67 & .130 \\
\hline Infl new supv & 6.65 & 5.51 & $20.69 \%$ & 8.145 & 1703 & .000 & 1.14 & .140 \\
\hline $\begin{array}{l}\text { Comp good } \\
\text { benefits }\end{array}$ & 5.58 & 6.83 & $-18.30 \%$ & -8.980 & 967 & .000 & -1.25 & .139 \\
\hline $\begin{array}{l}\text { Supv work/life } \\
\text { bal }\end{array}$ & 5.42 & 6.6 & $-17.88 \%$ & -7.555 & 906 & .000 & -1.18 & .157 \\
\hline $\begin{array}{l}\text { Supv clear } \\
\text { direction }\end{array}$ & 5.64 & 6.74 & $-16.32 \%$ & -7.672 & 954 & .000 & -1.09 & .143 \\
\hline Supv fair & 6.42 & 7.67 & $-16.30 \%$ & -9.639 & 1047 & .000 & -1.24 & .129 \\
\hline $\begin{array}{l}\text { Comp val } \\
\text { prdet/sve }\end{array}$ & 7.44 & 8.37 & $-11.11 \%$ & -8.911 & 1037 & .000 & -.93 & .105 \\
\hline $\begin{array}{l}\text { Infl better } \\
\text { company }\end{array}$ & 8.69 & 8.09 & $7.42 \%$ & 5.804 & 731 & .000 & .59 & .103 \\
\hline
\end{tabular}

Table 1.5.3 Significant differences between the means of seekers vs. passives

Significant Differences in Likelihood to Recommend

This section concludes with a brief discussion on the significant differences between the means of seekers, passives and non-seekers on likelihood to recommend their company.

As might be expected by now, all groups evidenced significant differences on this variable, with seekers and non-seekers evidencing the largest gap, nearly $45 \%(44.53 \%)$, seekers rating likelihood to recommend at 4.57 and nonseekers at 8.24

The difference between seekers and passives is substantial at 4.57 and 6.69 respectively, with passives rating likelihood to recommend nearly $32 \%$ higher $(31.69 \%)$.

Further, note the significant differences between passives and non-seekers, 6.69 and 8.24 , with non-seekers rating likelihood to recommend nearly $19 \%$ higher (18.81\%). Table 1.5.4 highlights these differences. 


\begin{tabular}{|c|c|c|c|c|c|c|c|}
\hline $\begin{array}{l}\text { Likelihood to } \\
\text { Recommend }\end{array}$ & Mean & $\begin{array}{l}\text { Percent } \\
\text { difference }\end{array}$ & T Stat & $\begin{array}{l}\text { Degrees } \\
\text { freedom }\end{array}$ & $\begin{array}{l}\mathrm{p} \text { - } \\
\text { value }\end{array}$ & $\begin{array}{l}\text { Mean } \\
\text { diff }\end{array}$ & $\begin{array}{l}\text { Std. } \\
\text { error } \\
\text { diff }\end{array}$ \\
\hline Seekers & 4.57 & $44.53 \%$ & -16.53 & 135 & .000 & -3.67 & .222 \\
\hline Non-seekers & 8.24 & & & & & & \\
\hline Seekers & 4.57 & $31.69 \%$ & -15.03 & 1697 & .000 & -2.11 & .150 \\
\hline Passives & 6.69 & & & & & & \\
\hline Passives & 6.69 & $18.81 \%$ & -6.57 & 171 & .000 & -1.55 & .237 \\
\hline Non-seekers & 8.24 & & & & & & \\
\hline
\end{tabular}

Table 1.5.4 Significant differences likelihood to recommend

Deficiency Leading to Misclassification

The important point to note as in closing this section is that the mean score for likelihood to recommend (loyalty) gives us insightful points of comparison, but it is deficient in classifying seekers, passives and non-seekers.

The standard deviation for each category is so large that members cannot effectively be classified based on individual scores for likelihood to recommend, which are discrete, 1 through 10. Table 1.5.5 summarizes the means and standard deviations for each category under investigation.

\begin{tabular}{|l|ll|}
\hline $\begin{array}{l}\text { Likelihood to } \\
\text { Recommend }\end{array}$ & Mean & $\begin{array}{l}\text { Standard } \\
\text { Deviation }\end{array}$ \\
\hline Seekers & 4.57 & 2.850 \\
\hline Passives & 6.69 & 2.483 \\
\hline Non-seekers & 8.24 & 2.098 \\
\hline
\end{tabular}

Table 1.5.5 Means and standard deviation by category

Therefore, a more reliable method for assessing loyalty is pursued. The aim is to utilize the information obtained from the nineteen key variables in a manner that facilitates the creation of a new, more scientific loyalty measure. 


\subsection{Conclusion of Part 1 - A Better Classification Method is Needed}

An organization is comprised of primarily three types of individuals; those that are not seeking a new position, content to stay in their current situation, those that would leave given the right opportunity, and those who are actively seeking an outside alternative while currently employed at the organization.

The analysis thus far enables one to understand some of the distinguishing characteristics of each group. Each group, or subclass, has been identified to be significantly different from the others on virtually all measures tested. These measures include attitudes regarding the current organization and supervisor, as well as the influences on one's decision to transition. These influences include perceptions of external opportunities and potential for improved quality of work-life.

A sense of company pride is a very distinguishing characteristic between groups, and is highly predictive of loyalty when loyalty is measured as likelihood to recommend the company. In turn, some of the issues and potential for misclassification based on loyalty measured in this way were discussed.

It has been revealed that, in addition to perceptions of company pride and honesty, the supervisor's role as a good mentor who supports an individual's work/life balance has the potential to transition an employee from state to state.

In Part 2, several techniques are investigated in an attempt to classify employees into job-seeking status categories. First, a "loyalty score" is built with the intention of classifying seekers, passives and non-seekers based on their loyalty score. Several classification and analysis techniques are discussed including factor analysis, cluster analysis, discriminant analysis, and finally a generalized additive model is fit to the job-seeker data. The nineteen variables measured are reduced to four critical factors, namely supervisor, company, opportunity, and quality of work-life. These factors are then used to fit a generalized additive model predicting the classification of employees into seeking status categories. 


\subsection{Definition of Loyalty Score}

Reasoning and Goal

The job turnover literature (see Section 1.1) suggests that multiple factors contribute to an employee's decision to leave the organization. Early research clarified that one's attitudes toward their job affects participation more than performance. Further, dissatisfaction is more likely to push an employee to consider alternatives, and attractive alternatives pull the employee toward transition out of the company (March and Simon, 1958). This sets a foundation for equating satisfaction with the one's job to satisfaction with the company and supervisor, both indicative of participation (i.e. staying in the company).

Recall that it was later shown that personality, the comparison standard, and perceptions of fairness all helped to distinguish subjects who stayed in the organization versus those who terminated (Atchison and Lefferts, 1972). An attempt to further validate these findings is made here with measurements for opportunity and quality of work-life. The literature later acknowledged the need to investigate external influences on one's decision to leave a company (Mobley et al, 1979). It was suggested that considering both satisfaction and external opportunities should increase understanding and prediction of turnover behavior. Researchers identified the need to distinguish between present-oriented satisfaction (e.g., company and supervisor) and futureoriented expectations for both the current situation and alternatives (e.g., opportunity and quality of work-life).

These suggestions provide sound reasoning for developing a mechanism for scoring the likelihood of turnover as reflected in a loyalty score. Further, such a score has the potential for identifying employees in a passive state, the need for which was suggested by Kirschenbaum and Weisberg (1994).

What is unclear from investigations of the withdrawal process in the literature is the appropriate amount of weight for each factor contributing to turnover. From a managerial perspective, it makes sense that satisfaction with one's supervisor, perhaps followed by or comparable to satisfaction with the company, would play a more important role in turnover than would external opportunities. Thus the goal of this section is to produce a scoring method that takes into consideration the relative importance of each of the elements potentially leading to transition. 
Method

In an effort to create a more scientific loyalty score, factor analysis is implemented as a means to reduce the variables in a way that reveals underlying constructs. The factors are weighted and then summed to create a loyalty score which differs significantly based on seeking status. The intent is to use the loyalty score in classifying seeking status.

The definition takes a weighted sum of the four factor scores. These weights are the amount of variance explained via principle components analysis, which is selected as the method of extraction within the factor analysis procedure in SPSS. The variance explained by each of the components is used as the weight for the factor. Descriptive statistics indicate that the first two weights, for supervisor and company, are taken positive and the second two, for opportunity and quality of work-life are taken negative. This gives a high loyalty score to non-seekers and a low score to seekers. Thus, the loyalty score is defined as

$$
L S=: 6.91 y_{1}+9.424 y_{2}-: .104 y_{3}-.769 y_{4}
$$

where $y_{1} \ldots y_{4}$ are the factor scores for the underlying constructs, namely supervisor, company, opportunity and quality of work-life. Details regarding the selection of methods, as well as justification of the loyalty score follow.

Factor Analysis with Principle Components Method of Extraction

In order to build the loyalty score from the nineteen questions, factor analysis is used with the data set of 1,817 currently employed respondents. The purpose of factor analysis is to describe covariance relationships among many variables, in terms of underlying, but otherwise unobservable random quantities, called factors (Johnson \& Wichern, 2001). Variables are grouped by their correlations, and kept independent, or only very slightly correlated. Each group represents and independent construct that is responsible for the observed correlations. Normality, which we do not have with the job-seeker data set, is not considered a critical assumption of factor analysis. The normality assumption is required for significance testing of coefficients, and with factor analysis we are interested in clustering, or correlating, groups of like variables into underlying constructs.

Several methods are available for extracting factors from a data set. Common factor analysis, also called principle axis factoring, is a correlation-focused approach to representing the common variance of the variables under investigation, excluding unique variance. The aim is to reproduce 
intercorrelations among variables. This method seeks the least number of factors which can account for the common variance (correlation) given the set of variables (Garson 2009). It is typically used for structure detection when one seeks to identify latent structures.

Alternately, the principle components method can be used as an extraction method. From principle components analysis, the idea is to build components that reflect both common and unique variance of the variables. This variancefocused approach seeks to reproduce both the total variable variance with all the components and to reproduce the correlation. It is typically used for data reduction.

To extract the first component, the principle component analysis method (an option within the factor analysis procedure in SPSS) generates a linear equation to extract the maximum total variance from the variables. To pull the second component, the PCA removes the variance explained by the first component and creates a second linear equation which extracts the maximum remaining variance, and so on. This continues until the components explain all the common and unique variance in the set of variables under investigation.

Common factor analysis is used for causal modeling, whereas the principle components analysis method of extracting factors is more appropriate for data reduction and exploration of underlying constructs. For most datasets, common factor analysis and principle components analysis will lead to similar substantive conclusions (Wilkinson, Blank, and Gruber, 1996).

\section{Applied to the Job-Seeker Data}

Given the data set of 1,817 employed respondents, common factor analysis (also called principle axis factoring) results in 53\% variance explained with four factors. Using principle components analysis as the extraction method results in the same variables loading on the same four components, called factors in common factor analysis) yet with more, $62 \%$, variance explained (Table 2.1.1). Thus, the principle components method is preferred in this application. 


\begin{tabular}{|c|c|c|c|c|c|c|c|c|c|}
\hline \multirow[b]{2}{*}{$\begin{array}{l}\text { Comp } \\
\text { onent }\end{array}$} & \multicolumn{3}{|c|}{ Initial Eigenvalues } & \multicolumn{3}{|c|}{$\begin{array}{l}\text { Extraction Sums of Squared } \\
\text { Loadings }\end{array}$} & \multicolumn{3}{|c|}{$\begin{array}{l}\text { Rotation Sums of Squared } \\
\text { Loadings }\end{array}$} \\
\hline & Total & $\mid \begin{array}{l}\% \text { of } \\
\text { Variance }\end{array}$ & $\begin{array}{l}\text { Cumulative } \\
\%\end{array}$ & Total & $\mid \begin{array}{l}\% \text { of } \\
\text { Variance }\end{array}$ & $\begin{array}{l}\text { Cumulative } \\
\%\end{array}$ & Total & $\begin{array}{l}\% \text { of } \\
\text { Varianc } \\
\text { e }\end{array}$ & $\begin{array}{l}\text { Cumulativ } \\
\text { e } \%\end{array}$ \\
\hline 1 & 5.113 & 26.910 & 26.910 & 5.113 & 26.910 & 26.910 & 3.247 & 17.088 & 17.088 \\
\hline 2 & 3.691 & 19.424 & 46.334 & 3.691 & 19.424 & 46.334 & 3.209 & 16.888 & 33.977 \\
\hline 3 & 1.540 & 8.104 & 54.439 & 1.540 & 8.104 & 54.439 & 2.692 & 14.166 & 48.143 \\
\hline 4 & 1.476 & 7.769 & 62.208 & 1.476 & 7.769 & 62.208 & 2.672 & 14.065 & 62.208 \\
\hline 5 & .880 & 4.631 & 66.839 & & & & & & \\
\hline 6 & .860 & 4.526 & 71.365 & & & & & & \\
\hline 7 & .771 & 4.056 & 75.421 & & & & & & \\
\hline 8 & .621 & 3.269 & 78.690 & & & & & & \\
\hline 9 & .596 & 3.135 & 81.824 & & & & & & \\
\hline 10 & .573 & 3.018 & 84.842 & & & & & & \\
\hline 11 & .458 & 2.413 & 87.255 & & & & & & \\
\hline 12 & .445 & 2.343 & 89.598 & & & & & & \\
\hline 13 & .376 & 1.980 & 91.578 & & & & & & \\
\hline 14 & .335 & 1.764 & 93.342 & & & & & & \\
\hline 15 & .319 & 1.678 & 95.020 & & & & & & \\
\hline 16 & .282 & 1.485 & 96.506 & & & & & & \\
\hline 17 & .237 & 1.250 & 97.755 & & & & & & \\
\hline 18 & .231 & 1.218 & 98.973 & & & & & & \\
\hline 19 & .195 & 1.027 & 100.000 & & & & & & \\
\hline
\end{tabular}

Table 2.1.1 Principal component extraction method suggests 4 components explain a total of $62.2 \%$ of variance in the data from 19 variables 
The component scores plotted in rotated space (Figure 2.1.1) provides graphic insight into the composition of the first three components. The company variables are clustered together, as are the supervisor variables, and the external influence variables, some related to opportunity, some quality of work-life.

\section{Component Plot in Rotated Space}

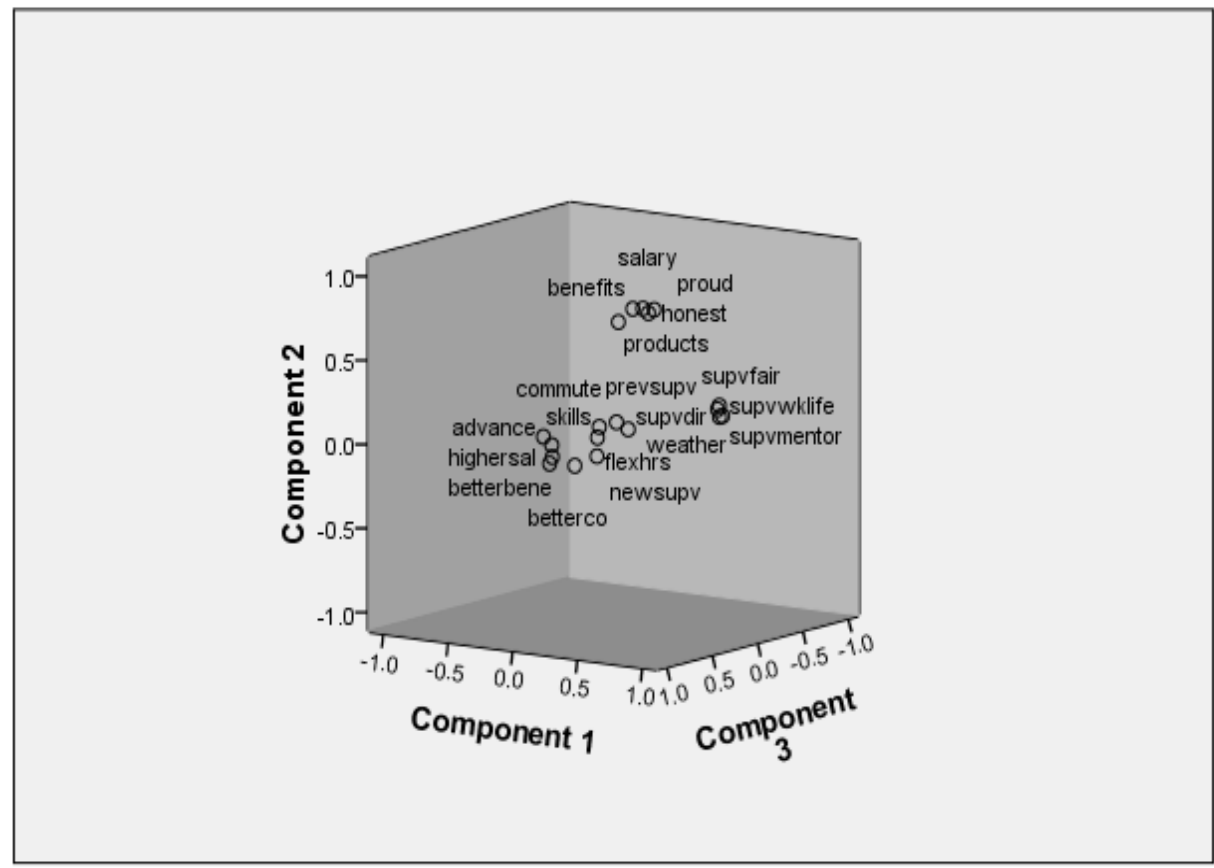

Figure 2.1.1 Component plot shows variables clustering into four components

As will be detailed in Section 2.1b, the researcher reviews the rotated component matrix to identify highest loading for each variable, given each component score (four scores for each variable), to determine the component on which the variable falls. Then, reviewing the collection of variables falling on an individual component, the researcher (somewhat subjectively) nicknames the component to reflect the underlying construct it represents. Thus the constructs are named supervisor (SUPV) for the variables falling on the first component which represent supervisor satisfaction-related variables; company (COMP) for those loading on component two and representing satisfaction with the company; opportunity (OPPTY) is represented by the third component and quality of work-life (QWL) by the fourth. See Table 2.1.7, the rotated component matrix for loadings which, when squared, represent the percent of variance in the indicator variable explained by the component. 
These components will hereafter be referred to by the name of the "factor" they represent (e.g., SUPV, COMP, OPPTY, QWL). However, it is reiterated that the components actually include small portions of variance explained by each of the indicators, some of which are not considered as "loading" on the component due to their very minor contribution.

The final factors are comprised as follows in Table 2.1.2

\begin{tabular}{|c|c|c|c|}
\hline Supervisor & Company & $\begin{array}{l}\text { Opportunity } \\
\text { (influencers of job } \\
\text { change) }\end{array}$ & $\begin{array}{l}\text { Quality of Work-Life } \\
\text { (influencers of job } \\
\text { change) }\end{array}$ \\
\hline 1. Treats me fairly & $\begin{array}{l}\text { 1. Provides valuable } \\
\text { products and services }\end{array}$ & 1. Higher salary & 1. Shorter commute \\
\hline $\begin{array}{l}\text { 2. Provides clear } \\
\text { direction }\end{array}$ & 2. Company is honest & $\begin{array}{l}\text { 2. Greater } \\
\text { advancement } \\
\text { opportunity }\end{array}$ & 2. Better weather \\
\hline $\begin{array}{l}\text { 3. Supports work-life } \\
\text { balance }\end{array}$ & 3. Competitive salary & 3. Better benefits & 3. Better company \\
\hline \multirow[t]{3}{*}{ 4. Good mentor } & 4. Good benefits & $\begin{array}{l}\text { 4. Better skills } \\
\text { training }\end{array}$ & 4. New supervisor \\
\hline & $\begin{array}{l}\text { 5. Proud to work at } \\
\text { company }\end{array}$ & & 5. Previous supervisor \\
\hline & & & 6. Flexible work hours \\
\hline
\end{tabular}

Table 2.1.2 Variables that load on each component

Reliability of the Factors

To validate the reliability of the factors, as if they were scales unto themselves, consider the both inter-item correlation, and Cronbach's coefficient of reliability (Cronbach, 1951). The goal is a scale that is internally consistent, yet each variable contributes unique information. The scale variance suggests larger variance within the supervisor and company factors (Table 2.1.3). This makes sense because seekers and non-seekers are likely to rate these elements quite differently as will be clarified in Section 2.2.

\begin{tabular}{|l|c|c|c|c|}
\hline $\begin{array}{l}\text { Factor } \\
\text { (\# items) }\end{array}$ & $\begin{array}{l}\text { Mean } \\
\text { inter-item } \\
\text { correlation }\end{array}$ & $\begin{array}{l}\text { Inter-item } \\
\text { variance }\end{array}$ & $\begin{array}{l}\text { Coefficient of } \\
\text { reliability for } \\
\text { scale* }\end{array}$ & Scale variance \\
\hline SUPV (4) & .722 & .001 & $\alpha=.91$ & 106.30 \\
\hline COMP (5) & .540 & .011 & $\alpha=.85$ & 111.11 \\
\hline OPPTY (4) & .551 & .010 & $\alpha=.83$ & 28.26 \\
\hline QWL (6) & .312 & .003 & $\alpha=.72$ & 95.82 \\
\hline
\end{tabular}

Table 2.1.3 Factors are reliable based on inter-item correlations and coefficient of reliability 


\section{Coefficient of Reliability - Cronbach's Alpha}

The coefficient, $\alpha$, is based on the number of items in the scale and the ratio of average inter-item covariance to the average item variance. Cronbach (Psycometrika, 1951) provides a general formula:

$$
\alpha=\frac{n}{n-1}\left(1-\sum v_{i}\right)
$$

Here, $v_{\mathrm{t}}$ is the variance of test scores, and $v_{\mathrm{i}}$ is the variance of the item scores after weighting. If the number of items is increased, Cronbach's alpha increases. If the average inter-item correlation is low, alpha will be low. As the average inter-item correlation increases, Cronbach's alpha increases as well. Typically, 0.6 to 0.8 indicates acceptable reliability, 0.8 or higher, good reliability, and at 0.95 or higher, the variables may be redundant. Garson (2009) suggests the widely accepted cut-off in the social sciences is .70, with some researchers using .75 to .80. Others are as lenient at .60. Garson notes that .70 is as low as one should go because at that point the standard error of measurement will be over half $(0.55)$ of a standard deviation.

Discussion

Four distinct factors have been extracted that reliably reflect perceptions of satisfaction with company and supervisor, as well as perceptions of external influences related to opportunity and potential quality of work-life. The principal components method of extraction suggests that the first component comprised of supervisor-related variables, accounts for nearly $27 \%(26.9 \%)$ of the variance explained. This large amount of explanatory strength supports findings by Brough and Frame (2004) and Eisenberger et al (2002) regarding the important role of supervisor in job satisfaction and turnover intent. Variables loading on this component included "fair treatment" (DeConick and Stilwell, 2001; Fields et al, 2000; Folgers and Konovsky, 1989: Konovsky and Cropanzano, 1991; Schaubroeck et al, 1994; Sweeney and McFarlin, 1993), "clear direction", which is discussed in the literature as role ambiguity (Acquino et al, 1997; Jackson and Schuler, 1985; James and James, 1989; Mathieu, 1991; Mathieu and Zajac, 1990), "supports work/life balance" (Cater and Buffardi, 2004; Beauregard and Henry,2009) and "good mentor" (Calkins, 1987). This component is given the factor name "supervisor".

The second component extracted, explains $19.4 \%$ of the variance, and represents company-related variables. This supports findings by DeConick and Stilwell (2001) and others regarding the importance of organizational justice in understanding employees' propensity to search (Berkowitz et al, 1987; 
Folger and Cropanzano, 1998; Folger and Konovsky, 1989; Greenberg, 1990; Sweeney and McFarlin, 1993). Variables including "company provides valuable products and services," company honesty and one's pride in working for the company fell on this second component as did competitive salary and good benefits. This component is given the factor name of "company".

A third component emerged, accounting for just over $8 \%(8.1 \%)$ of the variance, which reflects the external influences of alternate opportunities (Steers and Mowday, 1981). Variables loading on this component include the potential for higher salary, greater opportunity for advancement, and better benefits and skills training. Recall Mobley's work (1977) suggesting a need to distinguish between present-oriented satisfaction and future-oriented attraction/expected utility for both current job and alternatives. Further, Mobley et al (1979) suggest the value in such information in potentially predicting turnover intentions and behaviors. The component is named "opportunity" for the underlying construct, or factor, represented.

A final component, quality of work-life, accounts for close to an additional $7.77 \%$ of the explained variance. It is comprised of six variables reflecting exogenous influences such as shorter commute, better weather, better company, chance to work with a previous or new supervisor, and flexible work hours. Recall Cater and Buffardi's work (2004) which found that benefits such as schedule flexibility and dependent care assistance contribute uniquely to predicting anticipated organizational support and job pursuit intentions. This component is given a factor name of "quality of work-life."

Considering that the four factors extracted are representative of facets addressed in the literature, and some were suggested for future research, it is appropriate to move forward with the factor scores in the development of a loyalty score, potentially indicative of job-seeking status. Assigning weights to the factors makes sense from a managerial perspective because an employer can more realistically control company and supervisor perceptions, whereas external variables such as perceptions of alternate opportunities and the potential for better quality of work-life are influences typically beyond the control of management.

Previous research has highlighted the importance of the supervisor in turnover intent, and the supervisor factor would be expected to be more influential than other facets (thus receiving heavier weight). The literature also clarifies the importance of the employee's perception of organizational justice and the company factor clearly captures this information. The final two factors serve to "pull" employees from the organization (March and Simon, 1958) and should be negatively weighted proportionate to the variance they explain. 
Thus the research proceeds with weights assigned to the factors which are representative of their importance and direction of influence. The weights assigned are the amount of variance explained by the factor which makes sense from a managerial perspective and is supported by the turnover literature.

\section{1a Justification of Loyalty Score}

Each of the components (i.e., SUPV, COMP, OPPTY and QWL) of the loyalty score are weighted by the variance explained based on the principle component method of extraction. To do this, several assumptions have been met with regard to principal component analysis (PCA). PCA is used in exploratory data analysis and can be applied under any distribution, thus it is a nonparametric approach. It gives a new basis for the vectors in the dataset where the coordinates have decreasing variability. A basis is sought, which is a linear combination of the (nineteen) original variables, that best expresses the data in fewer dimensions. Thus, linearity is implicit, simplifying the problem by restricting the set of potential bases (Shlens 2010). A second assumption is the statistical importance of the mean and variance as sufficient statistics, describing the probability distribution. While deviations from normality can invalidate this assumption, the covariance matrix effectively characterizes the noise and redundancies. Further, statistical independence is the most rigorous form of eliminating redundancy, and PCA de-correlates the axes of the coordinate system.

Perhaps the most valuable assumption, given this application, is that large variances have important dynamics. This is precisely the justification for taking the weights from the principal components analysis method of extraction within the factor analysis procedural options.

Examining the questions contributing to the single factor scores, it is clear from the managerial viewpoint that some questions clearly give more information than others. Moreover, the questions for supervisor and company give high scores to non-seekers as one expects, while these same questions will be rated lower by seekers. It is expected that the influences of alternate opportunities and potentially better quality of work life will get higher scores from seekers. This intuitive reasoning is also supported by the descriptive analysis of the loyalty score. However, some questions in the dataset may be adding more noise than value, specifically, those comprising the quality of work-life factor.

Consider the variance of the loyalty score for all data combined, $\sigma=5,221$.

By subclass, variance is substantially reduced with $\sigma_{\text {eker }}=4,503$, 
$\sigma_{\text {sssive }}=1,775$, and $\sigma_{m-s e e \text { ker }}=i, 576$ (Table 2.1.4). This supports the suggestion that the loyalty score has the potential to aid in the classification of employees by seeking status.

An understanding of the variability within the components adds justification to the weighting of components of the loyalty score. Compare the reduction in variance from the whole dataset to each subclass, then compare between subclasses. As stated above, larger variances indicate more important dynamics, whereas limited differences suggest potential noise.

\begin{tabular}{|lllll|}
\hline VARIANCE & All data & Seeker & Passive & Non-seeker \\
\hline $\begin{array}{l}\text { Loyalty } \\
\text { Score }\end{array}$ & 15221 & 14503 & 9775 & 6576 \\
\hline SUPV & 9.98 & 10.51 & 7.07 & 4.25 \\
\hline COMP & 8.57 & 8.28 & 5.51 & 3.82 \\
\hline OPPTY & 2.61 & 2.47 & 2.26 & 5.29 \\
\hline QWL & 6.72 & 6.30 & 6.84 & 9.06 \\
\hline
\end{tabular}

Table 2.1.4 Comparison of variances within full dataset vs. subclasses

The supervisor factor evidences substantial variances between the subclasses, also when each are compared to the entire dataset. Recall, $70 \%$ of the respondents classified themselves as seekers so it might be expected that the seeker variance is similar to the full dataset.

The company factor suggests reduced variability within the individual subclasses versus the full dataset, and each is distinguishable from the others given its variance.

For opportunity and quality of work-life, variance is lower in the subclasses compared to the entire dataset, with the exception of non-seekers. The forum for responding to the survey must be considered: an online job-search engine. This means that we may have respondents reporting themselves as nonseekers, yet quite varied in their responses regarding outside influences. This behavior may be indicative of underlying preferences to seek a new position. Again, this may be an effect of the administration of the survey (online jobsearch site). In practical application however, non-seekers may evidence more homogeneous behaviors. At this point, the related questions may be adding more noise than value.

Consider Levene's test for homogeneity of variances. The null of homogeneity between subclasses can be rejected at $p<.01$, with the exception of seekers 
and passives, on OPPTY and QWL. Further, the null is rejected at only $p<$ .10 for QWL between passives and non-seekers.

\begin{tabular}{|l|c|c|c|c|c|c|}
$\begin{array}{l}\text { Homogeneity } \\
\text { of variances }\end{array}$ & $\begin{array}{l}\text { Seeker } \\
\text { vs. } \\
\text { Passive }\end{array}$ & \multicolumn{3}{c}{$\begin{array}{l}\text { Passive vs. } \\
\text { Non-seeker }\end{array}$} & \multicolumn{3}{c|}{$\begin{array}{l}\text { Seeker vs. } \\
\text { Non-seeker }\end{array}$} \\
\hline & F & Sig. & F & Sig. & F & Sig. \\
\hline SUPV & 42.1 & .000 & 12.7 & .000 & 47.07 & .000 \\
\hline COMP & 30.0 & .000 & 8.1 & .005 & 29.61 & .000 \\
\hline OPPTY & .318 & .574 & 17.8 & .000 & 18.16 & .000 \\
\hline QWL & .441 & .507 & 3.5 & .061 & 6.16 & .013 \\
\hline
\end{tabular}

Table 2.1.5 Levene's test suggests variances are not equal with the exception of OPPTY and QWL (seeker vs. passive); $p<.10$ for QWL passive vs. non-seeker

A score that indicates an employee's loyalty to the company would be beneficial to managers. Understanding an employee's loyalty can also be considered a measure of the likelihood of the employee seeking a new job. Examples of applications for a loyalty score follow.

- If a reduction in forces is required, a company would prefer to cut its less loyal employees, and retain those with a stronger commitment to the organization.

- A series of scores reflecting the proportions of seekers, passives and non-seekers within an organization, is indicative of the stability of its human resources, perhaps even morale to an extent.

- Maintaining a high proportion of seekers is indicative of a less stable organization, whereas maintaining a substantial proportion of satisfied non-seekers intuitively suggests a more dynamic culture and higher potential for growth.

- While passives are more challenging to identify, this group can potentially drain resources such as training and development without the returning the value of organizational commitment.

Further, it should be noted that one of the challenges addressed is generating such a score without directly asking the employee his/her seeking status. The method discussed enables the employer to pose nineteen questions that are used to compose the loyalty score which is in turn used to determine the probability that the employee is seeking alternative employment. The questions concerning quality of work-life should be restructured to increase variability between subclasses. 


\section{1b Building the Loyalty Score}

Nineteen new "component score" variables were computed for each case that are a weighted representation of the response. Specifically,

$$
\left.C S_{x_{i}}=\alpha i_{i}\right)
$$

where the $\alpha$ are the highest-loading coefficients for the variable. For example, in the first line of the component score coefficient matrix (Table 2.1.6), the variable "current company provides valuable products and services" has a coefficient of .271 that is the highest of the component scores, and loads on the second component, as do the other company related variables.

\begin{tabular}{|c|c|c|c|c|}
\hline \multicolumn{5}{|c|}{ Component Score Coefficient Matrix } \\
\hline & \multicolumn{4}{|c|}{ Component } \\
\hline & 1 & 2 & 3 & 4 \\
\hline $\begin{array}{l}\text { curr company provides valuable } \\
\text { product/svc }\end{array}$ & -.090 & .271 & .103 & -.086 \\
\hline curr company is honest & -.038 & .261 & .030 & -.049 \\
\hline curr company competitive salary & -.101 & .292 & -.080 & .073 \\
\hline curr company offers good benefits & -.127 & .303 & -.071 & .079 \\
\hline Proud to work at my company & -.027 & .263 & .016 & -.028 \\
\hline infl higher salary & .021 & -.025 & .374 & -.140 \\
\hline infl better benefits & .016 & -.038 & .350 & -.095 \\
\hline infl greater advancement oppty & -.056 & .044 & .302 & -.042 \\
\hline infl better skills training & -.049 & .020 & .207 & .074 \\
\hline infl location shorter commute & -.016 & .028 & -.013 & .220 \\
\hline infl location better weather & .001 & .025 & -.084 & .258 \\
\hline infl better company & -.013 & -.046 & .053 & .190 \\
\hline $\begin{array}{l}\text { infl chance to work with previous } \\
\text { supv }\end{array}$ & .022 & -.002 & -.149 & .316 \\
\hline infl chance to work with new supv & -.023 & -.033 & -.118 & .333 \\
\hline infl flex work hours & .000 & .001 & .000 & .220 \\
\hline supv treats me fairly & .306 & -.079 & .008 & -.033 \\
\hline supv provides clear direction & .322 & -.102 & -.011 & .011 \\
\hline supv supports wrk/life balance & .292 & -.068 & -.030 & .000 \\
\hline supv is a good mentor & .318 & -.100 & -.037 & .028 \\
\hline
\end{tabular}

Table 2.1.6 Component scores used as weights on each of respondent's 19 variable scores

These new component score variables were then summed to create a new "component score factor" comprised of the variables that loaded on the factor (see Table 2.1.6). For example,

$$
\left.C S_{\text {company }}=\sum C S_{\text {value }}+. .+C S_{\text {proud }}\right)
$$




\section{Determining Number of Factors and Defining Factor Scores}

Table 2.1.7 highlights the variables loading on each of the components as found in the rotated component matrix which offers easy interpretation. Names for the underlying constructs are assigned to each of the components and include $y_{1}=$ "supervisor" for the first component, $y_{2}=$ "company" for the second, $y_{3}=$ "opportunity" for the third and $y_{4}=$ "quality of work-life" for the final component.

\section{Rotated Component Matrix}

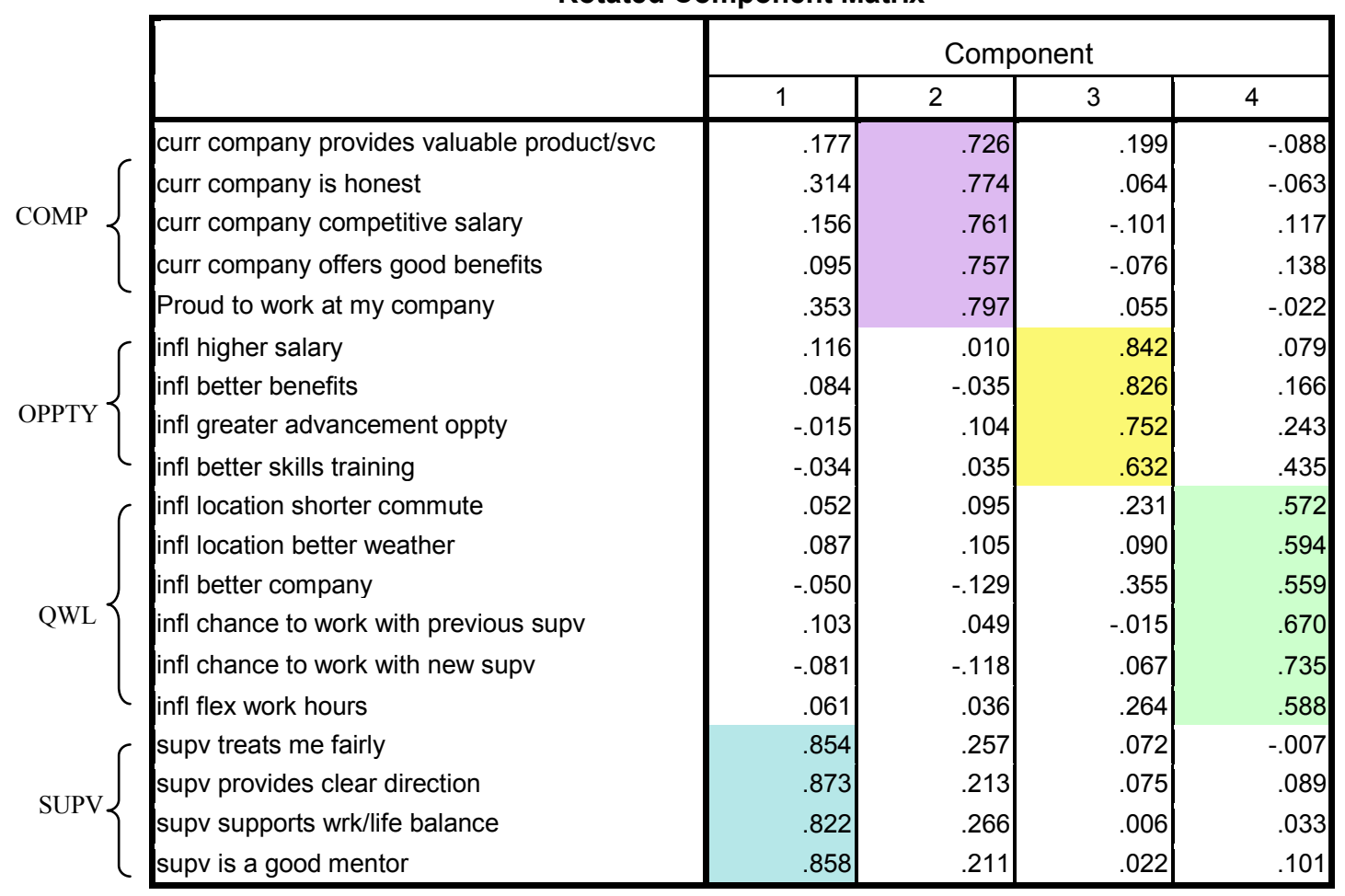

Table 2.1.7 Indicator variable loadings from principle components extraction method

The loadings are the correlation coefficients between the variables and the components (called factors in common factor analysis). Like Pearson's $r$, the squared loading is the percent of variance in the indicator variable explained by the component. Garson (2009) suggests a relatively high standard of .7 which corresponds to nearly half of the variance in the indicator variable being explained by the factor. Hair et al (1998) consider loadings above .6 as high and below .4, low.

Orthogonal transformation of the loadings is referred to as factor rotation because orthogonal transformations coincide with a rigid reflection of the coordinate axes (Johnson \& Wichern, 2001). Loadings obtained by an orthogonal transformation have the ability to reproduce the correlation matrix. 
The objective is to rotate the component axes to maximize the variance of the squared loading of a component on all the variables in the component matrix. The result is an easily-interpretable matrix with variables that have suitably high loadings for one of the components compared to low loadings on the others. The varimax orthogonal rotation method was specified in the generation of the components (Kaiser, 1958).

Another criterion that influences the number of components extracted is the Kaiser criterion. The Kaiser rule is to drop components with eigenvalues under 1.0. When this rule is relaxed to 0.8 , on the job-seeker data, six components emerge, resulting in $71.3 \%$ variance explained, an improvement over $62.2 \%$ with four components. Another check is done using the Cattell scree plot (Figure 2.1.2) which suggests that the decline in eigenvalues stops with the fifth component (for which the eigenvalue is 0.88). Cattell's scree test says that when the drop ceases and the curve makes an elbow toward less steep decline, drop further components after the one starting the elbow (Garson, 2009).

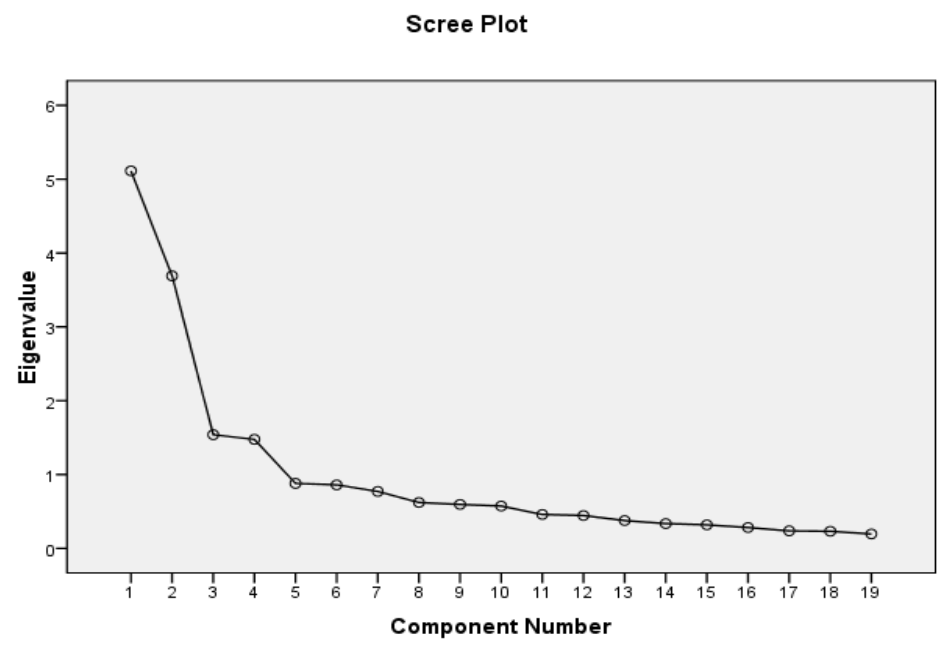

Figure 2.1.2 Cattell scree plot suggests five components

However, the complexity of using five or six variables in application is not deemed worth the price of variance explained and we proceed with the fourcomponent model which is supported by the Kaiser rule of extracting only components with eigenvalues greater than or equal to 1 . The final factors, $\left(y_{1} \ldots y_{4}\right)$, are then composed using weights from the component score matrix. Given the literature discussed earlier (Section 1.1 and Section 2.1), the weights are logical from a managerial perspective based on what is known about the elements affecting job search and turnover (Table 2.1.8) 


\begin{tabular}{|c|c|c|}
\hline$y_{1}$ & Supervisor & $(.306 *$ fair $)+(.322 *$ direction $)+(.292 *$ balance $)+(.318 *$ mentor $)$ \\
\hline$y_{2}$ & Company & $\begin{array}{l}(.271 * \text { products })+(.261 * \text { honest })+(.292 * \text { salary })+(.303 * \text { benefits })+ \\
(.263 * \text { proud })\end{array}$ \\
\hline$y_{3}$ & Opportunity & $(.374 *$ infl salary $)+(.350$ infl benefits $)+(.302$ infl advc $)+(.207$ infl train $)$ \\
\hline$y_{4}$ & $\begin{array}{l}\text { Quality of } \\
\text { Work Life }\end{array}$ & $\begin{array}{l}(.220 * \text { commute })+(.258 * \text { weather })+(.190 * \text { better co })+(.316 * \text { prev supv })+ \\
(.333 * \text { infl new supv })+(.330 * \text { infl flex })\end{array}$ \\
\hline
\end{tabular}

Table 2.1.8 Final component score factors composed with component score coefficients

\section{1c Descriptive Statistics by Seeking Status}

Next, the mean scores for the four factors are investigated (Table 2.1.9). It is expected that scores for supervisor and company would be higher for nonseekers and lower for seekers. In effect, the non-seeker is expected to be more satisfied with his current situation, likely to rate the supervisor and company higher, and thus not seeking a new position outside the company.

On the other hand, one might expect seekers to be more influenced by external opportunities and potential for a better quality of work-life. Thus, the seeker is expected to rate these influences higher, but the influences impact loyalty in a negative way, so the loyalty score should reflect this negative effect.

\begin{tabular}{|llcccc|}
\hline & & Seeker & Passive & Non-seeker & \\
\hline$y_{1}$ & Supervisor & 7.4 & 8.9 & 10.15 & \} \\
\hline$y_{2}$ & Company & 8.13 & 10.21 & 11.16 & \} \\
\hline$y_{3}$ & Opportunity & 10.91 & 10.75 & 10.09 & $\square$ \\
\hline$y_{4}$ & $\begin{array}{l}\text { Quality of } \\
\text { Work Life }\end{array}$ & 10.84 & 10.18 & 9.7 & $\square$ \\
\hline
\end{tabular}

Table 2.1.9 Mean scores for factors that comprise loyalty score; Opportunity and QWL negative

\section{1d. Definition of Loyalty Score and Descriptive Statistics}

The loyalty score, defined as

$$
L S=: 6.91 y_{1}+9.424 y_{2}-: .104 y_{3}-' .769 y_{4}
$$

is thus comprised of four factors, $y_{1} \ldots y_{4}$, weighted by the variance they explain as components extracted by the principle components analysis method 
within the factor analysis procedure in SPSS. The third and fourth factors are taken negative because they influence loyalty in a negative way.

The mean loyalty score for a seeker is 186.71 , increasing to 274.22 for the passive and 343.94 for the non-seeker. Descriptive statistics for the loyalty score and its components will be discussed in Section 2.2.

2.1e. Estimations of an unknown sample series of subclasses

Let $\quad N$ be a known sample series of LS

Let $\quad x$ be the number of seekers

$y$ be the number of passives

$z$ be the number of non-seekers among the $N$ persons.

Then we propose the following method to estimate $x, y$ and $z$.

$(1=$ seeker

Take dataset $(s, \xi$, where $\zeta=\mathrm{LS}$, and $s=2=$ passive

$$
3=\text { non-seeker }
$$

There are 1058 seekers, 411 passives, and 93 non-seekers in the dataset, with total $N=1562$.

The number of seekers, passives and non-seekers among those with scores greater than or equal to 301 is:

$$
200 \text { seekers } \quad 181 \text { passives } \quad 70 \text { non-seekers. }
$$

The number of seekers, passives, and non-seekers among those with scores less than or equal to 280 is:

801 seekers $\quad 180$ passives 18 non-seekers. 
This means that

$$
*\left\{\begin{array}{l}
19 \% \text { of all seekers have a score greater than or equal to } 301 \\
44 \% \text { of all passives have a score greater than or equal to } 301 \\
75 \% \text { of all non-seekers have a score greater than or equal to } 301 \\
76 \% \text { of all seekers have a score less than or equal to } 280 \\
46 \% \text { of all passives have a score less than or equal to } 280 \\
19 \% \text { of all non-seekers have a score less than or equal to } 280
\end{array}\right.
$$

In order to derive an estimate for $x, y$ and $z$ (defined above) we have 3 equations, assuming the percentage in $*$ persists.

Let $\mathrm{N}(280)=n$ people scoring less than or equal to 280 .

Let $\mathrm{N}(301)=n$ people scoring greater than or equal to 301 .

$$
\begin{gathered}
x+1+:=\mathrm{V} \\
0.76 x+1.46 y+1.19 z=\mathrm{V}(280) \\
0.19 x+1.44 y+1.75 z=\mathrm{V}(301)
\end{gathered}
$$

Solving these three equations for the unknowns $x, y$, and $z$ yields

$$
\begin{aligned}
& x=12.157 N(280)+10.588 N(280)-10.251 N \\
& y=3.704 N(280)-0.704 N-2.111 x \\
& z=V-;-
\end{aligned}
$$

Since $x, y$, and $z$ have to be integers, take the nearest integer to this solution.

Inserting $N=1580, N(280)=1030$ and $N(300)=450$, one detects

$x=1090$

$y=401$

$\mathrm{z}=89$,

a fairly close approximation to the dataset. 
From this we can estimate the probabilities of misspecification of a randomly chosen person with a score less than or equal to 280 .

$$
\begin{aligned}
P(\text { non-seeker } \mid \text { score } \leq 280) & =\quad \frac{\# \text { of non-seekers } \leq 280}{\text { total number of non-seekers }} \\
& =\frac{0.19 z}{\mathrm{~N}(280)}
\end{aligned}
$$

which is less than 2 percent.

$$
P(\text { non-seeker } \mid \text { score } \geq 301)=\frac{0.75 z}{\mathrm{~N}(301)}
$$

which is between 15 and 16 percent, not a good rate for detecting non-seekers.

Discussion

However, based on data inspection, if one says that a randomly selected person a non-seeker because his/her score was in the top 93 of the ordered set (the total number of non-seekers in the data), there is a $30 \%$ chance of accurate classification as a non-seeker. On the other hand, there is only a $2 \%$ chance that the person is actually a seeker. This classification is not as precise as desired, and the rest of the section discusses methods to improve it.

Using the loyalty score method, $75 \%$ of the non-seekers were identified among those with scores over 300. While the loyalty score gives more information, it is not complete. The loyalty score serves as a first and quick overview of the dataset. It is not good for specifying and predicting whether a single person is a seeker or a non-seeker. It only gives the overall picture. Analyzing the seeking status of each person is tedious and expensive in each case. 


\subsection{Descriptive Statistics of Loyalty Score and its Components}

Reasoning and Goal

An investigation of the descriptive statistics for the loyalty score and its components clearly suggests that the data is not normally distributed. However, as will be discussed next in Section 2.3, there are multiple significant differences between the means of the subclasses with regard to the loyalty score and its components. This section will underscore the finding that turnover intentions and the attitudes and behaviors related to them, cannot be evaluated using the normal distribution. While linear models under the normal distribution may not be suitable, nonparametric approaches offer reasonable alternatives to understanding differences and predicting classification. The usefulness of evaluating subclass differences based on asymptotic normality will be addressed in Section 2.3 .

Non-Normality of Loyalty Score and Factors

In the previous section, factor analysis, with the principal components method of extraction, was used to reduce a nineteen-dimensional vector into four components, each representing an independent construct that potentially influences job-seeking status. Factor score coefficients were used to create new variables that take into consideration the appropriate weights of each variable as loaded on the appropriate factor (Harman, 1979). These scores were then summed to create the loyalty score.

Checking for the normality of each factor as a new variable, as well as the new loyalty score, Shapiro-Wilk's test for normality is reported and a graphic examination conducted which includes a quantile-quantile plot, boxplot, histogram, and density plot.

For the Shapiro-Wilk's test, the $W$ statistic is calculated as follows:

$$
W=\frac{\left(\sum_{i=1}^{n} \alpha i_{(i)}\right)^{2}}{\sum_{i=1}^{n}\left(x_{i}-\bar{x}\right)^{2}}
$$

Where the $x_{i}$ are the ordered sample values $\left(x_{(1)}\right.$ is the smallest) and the $\alpha$ are constants generated from the means, variances and covariances of the order statistics of a sample of size $n$ from a normal distribution (Royston, 1995; Pearson and Hartley, 1972). 
Results from Shapiro-Wilk's statistical test for normality suggest that neither the factors nor the loyalty score evidence a normal distribution (Table 2.2.1). The $p$-values, all essentially zero, require that we reject the null hypothesis of normality.

\begin{tabular}{|lcc|}
\multicolumn{1}{c}{ Factor } & W-statistic & $\mathrm{p}$-value \\
\hline SUPERVISOR (SUPV) & .9494 & $p=0$ \\
\hline COMPANY (COMP) & .9751 & $p=0$ \\
\hline $\begin{array}{l}\text { OPPORTUNITY } \\
\text { (OPPTY) }\end{array}$ & .8061 & $p=0$ \\
\hline $\begin{array}{l}\text { QUALITY OF WORK- } \\
\text { LIFE (QWL) }\end{array}$ & .9769 & $p=0$ \\
\hline Loyalty Score (LS) & .9801 & $p=0$ \\
\hline
\end{tabular}

Table 2.2.1 Shapiro-Wilk's test suggests non-Gaussian distributions

Figure 2.2.1 through Figure 2.2.4 provide normality plots for each of the factors.. Keep in mind that the dataset is comprised of three very distinct types of respondents who differ significantly on most measures assessed.
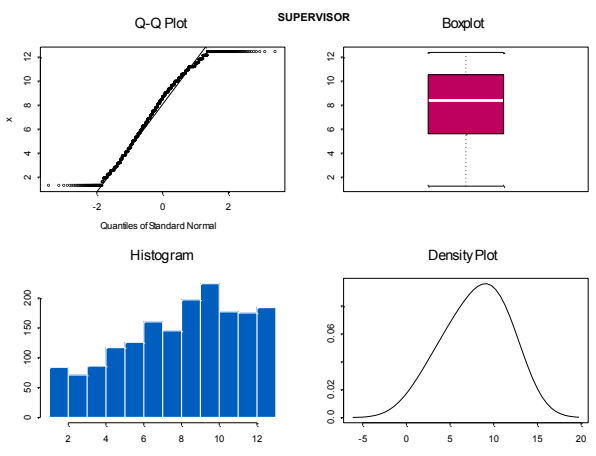

Figure 2.2.1 SUPV not normally distributed

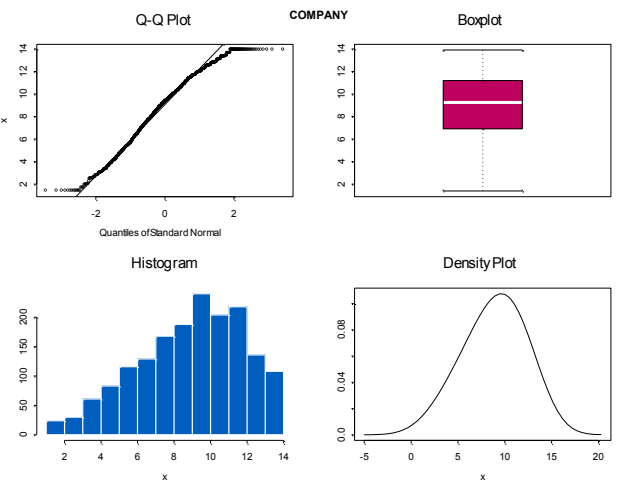

Figure 2.2.2 COMP not normally distributed 

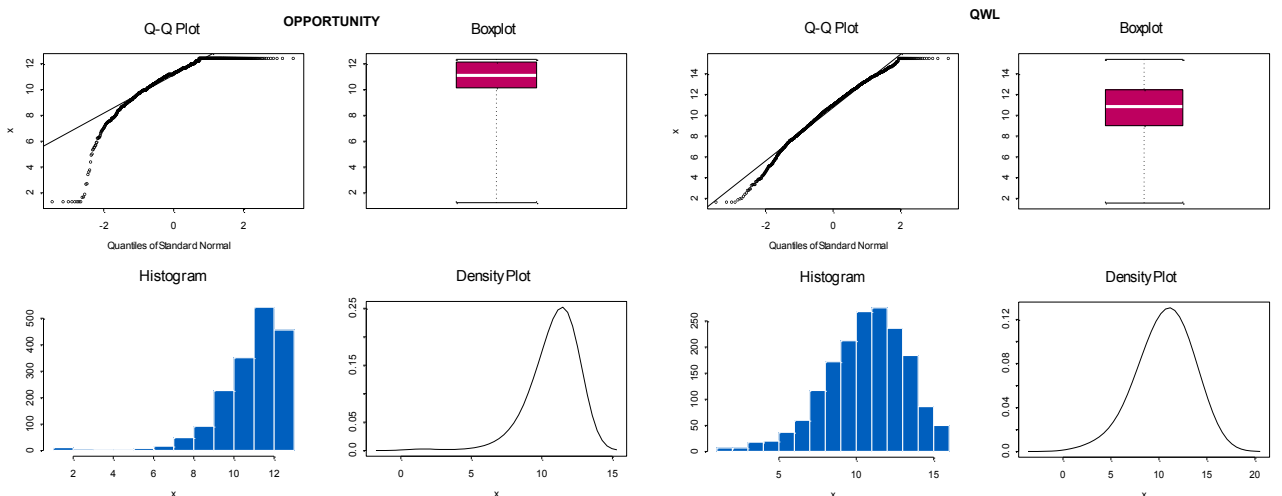

Figure 2.2.3 OPPTY not normally distributed
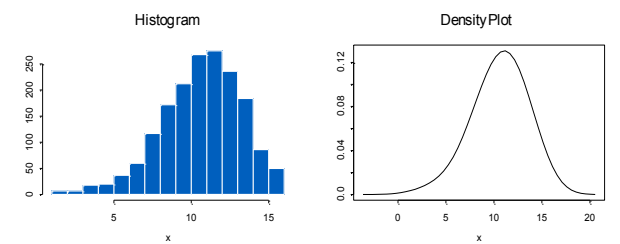

Figure 2.2.4 QWL not normally distributed

Table 2.2.2 provides a summary of the descriptive statistics for the factors and the new loyalty score. In Table 2.2.3, skewness and kurtosis statistics are presented. A negative left skew is strongest for the OPPTY factor which supports the suggestion that even non-seekers are potentially influenced by the external alternatives, namely the opportunity to work for a better company, with higher salary and better benefits. The kurtosis for OPPTY is quite leptokurtic, with $\gamma=i .19$. The SUPV and COMP factors do not evidence as many outliers as do OPPTY and QWL.

\begin{tabular}{|l|l|l|l|l|l|l|l|}
\hline Descriptive Statistics & N & Range & Min & Max & Mean & $\begin{array}{l}\text { Std. } \\
\text { Deviati } \\
\text { on }\end{array}$ \\
\hline & Statistic & Statistic & Statistic & Statistic & Statistic & $\begin{array}{l}\text { Std. } \\
\text { Error }\end{array}$ & Statistic \\
\hline SUPERVISOR & 1746 & 11.14 & 1.24 & 12.38 & 7.94 & .075 & 3.15 \\
\hline COMPANY & 1705 & 12.51 & 1.39 & 13.90 & 8.87 & .070 & 2.92 \\
\hline OPPORTUNITY & 1761 & 11.10 & 1.23 & 12.33 & 10.82 & .038 & 1.61 \\
\hline $\begin{array}{l}\text { QUALITY WORK } \\
\text { LIFE }\end{array}$ & 1749 & 13.83 & 1.54 & 15.37 & 10.60 & .062 & 2.59 \\
\hline \begin{tabular}{l} 
LOYALTY SCORE \\
\hline
\end{tabular} & 1562 & 715.09 & -157.95 & 557.13 & 219.10 & 3.12 & 123.26 \\
\hline
\end{tabular}

Table 2.2.2 Descriptive statistics for factors and loyalty score 


\begin{tabular}{|l|l|r|r|r|r|}
\hline \multicolumn{7}{|l|}{ Descriptive Statistics } & \multicolumn{5}{l|}{} \\
\hline & $\mathrm{N}$ & \multicolumn{1}{l|}{ Skewness } & \multicolumn{1}{l|}{ Kurtosis } \\
\hline & Statistic & \multicolumn{1}{l|}{ Statistic } & \multicolumn{1}{l|}{ Std. Error } & Statistic & Std. Error \\
\hline SUPERVISOR & 1746 & -.432 & .059 & -.812 & .117 \\
\hline COMPANY & 1705 & -.401 & .059 & -.541 & .118 \\
\hline OPPORTUNITY & 1761 & -2.21 & .058 & 8.18 & .117 \\
\hline QUALITY WORK-LIFE & 1749 & -.590 & .059 & .377 & .117 \\
\hline LOYALTY SCORE & 1562 & -.443 & .062 & -.334 & .124 \\
\hline
\end{tabular}

Table 2.2.3 Skewness and kurtosis for factors and loyalty score

\section{Loyalty Score}

Consider the loyalty score comprised of the four factors discussed above. The loyalty score has a wide range, from a minimum of -157.95 , to a maximum of 557.13 , with a mean of 219.10, and standard deviation, $\sigma=123.26$.

There is a heavy upper tail evidenced clearly the Q-Q plot (Figure 2.2.5). Skewness, $\gamma=-143$, and kurtosis $\gamma=334$, thus the general normality of the score is not reasonable (recall Shapiro-Wilk's $=.9801, p=0$, Table 2.2.1).
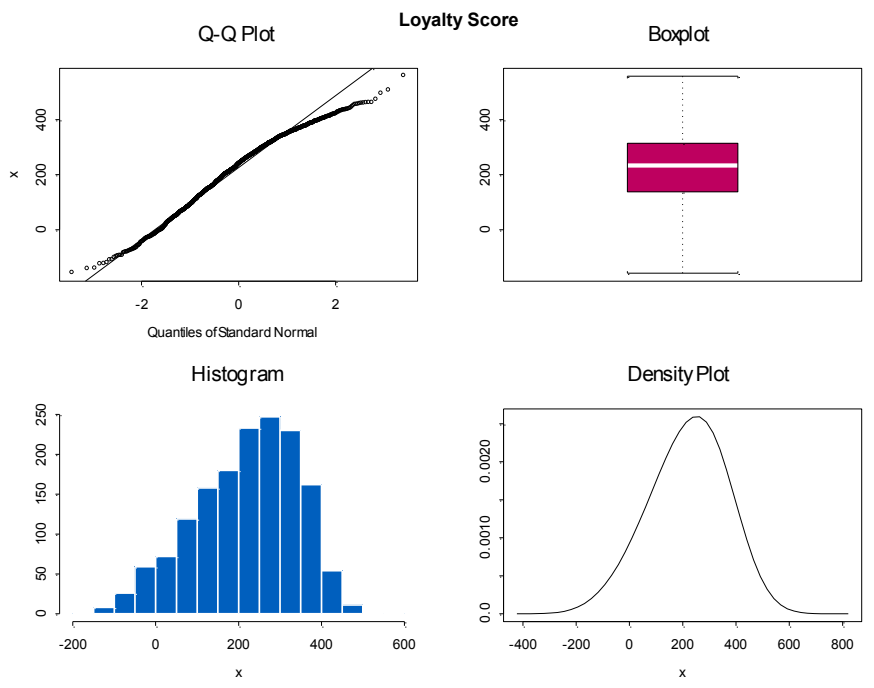

Figure 2.2.5 LS cannot be considered normally distributed

Overall the factors and the loyalty score evidence serious departures from normality. This is supported by the Shapiro-Wilk's tests as well in the graphic review of the distributions of each of the factors. 
This dataset complicates the suggestion that one might survey an organization and be able to differentiate seeking status of the employees based on the questions, factors, and ultimately, the loyalty score. However, we move forward using results from the factor analysis (where normality is not required), apply discriminant analysis techniques for classification, which are robust against departures from normality, and ultimately arrive at an effective nonparametric approach, the generalized additive model, to identifying seeking status.

Next, in Section 2.3, the three subclasses of seekers, passives, and non-seekers are investigated to better understand the variations suggested by the descriptive statistics for each factor and the loyalty score. 


\subsection{Descriptive Statistics of Factors by Subclass}

Reasoning and Goal

In this section, the three subclasses are investigated for normality and significant differences between the subclasses are examined. While the subclasses do not reflect normal distributions with regard to the factors or the loyalty score, there is valuable insight to be gained from the significant differences identified between the means of the varied seeking status categories (seekers, passives and non-seekers). These mean differences are identified using $t$-tests which are robust against non-normality with large sample sizes (PROPHET StatGuide, 1997). A proof of the asymptotic normality of the $t$ statistic is provided in Ferguson (1996). The Kruskal-Wallis one-way analysis of variance by ranks also validates the significance of differences between the three subclasses with a nonparametric test. However, this test assumes identically shaped and scaled distributions for each group. It is known from Section 2.1 (Table 2.1.5) that Levene's test for homogeneity of variances suggests we have unequal variances. The results of this test are provided in Appendix A, yet the violation of the identically-scaled assumption leads to the preference for using the $t$-tests, given asymptotic normality.

Recall that seekers are those who reported that they were currently seeking a new job at the time of the survey. Those considered passives reported that they would leave their current job, given the right opportunity. And the final group is comprised of non-seekers who said they were not currently seeking, and intent on staying in their current job for a while.

Seekers comprise $68.6 \%$ of the sample of full-time employed respondents $(\mathrm{n}=$ 1246), passives are $25.7 \%$ with $\mathrm{n}=467$, and non-seekers are the smallest group at $5.7 \%$, with $\mathrm{n}=104$. The data does not differentiate respondents who are seeking a new position within their current company. The online survey question was asked specifically as follows in Table 2.3.1, where the respondent clicked on the response radial button representing their current status:

\begin{tabular}{|c|c|}
\hline \multicolumn{2}{|c|}{ Are you currently seeking a new job opportunity? } \\
\hline o & Yes, actively seeking \\
\hline $\mathrm{o}$ & No, staying in my current job for a while \\
\hline $\mathrm{o}$ & $\begin{array}{l}\text { No, but would consider a new job if it were the } \\
\text { right opportunity }\end{array}$ \\
\hline
\end{tabular}

Table 2.3.1 Self-reported seeking status categories 
Non-Normality within the Subclasses

Shapiro-Wilk's test for normality was conducted on the distributions for the factors and loyalty scores based on seeking status. Again the test requires rejection of the null that the distributions are normal. With the exception of the QWL factor for non-seekers, all achieve an approximate $p$-level $=0$, indicating non-Gaussian distributions (Table 2.3.2).

\begin{tabular}{|llcc|}
\hline & Factor & W-statistic & p-value \\
\hline Seeker & SUPERVISOR & .958 & $p=0$ \\
\hline & COMPANY & .985 & $p=0$ \\
\hline & OPPORTUNITY & .800 & $p=0$ \\
\hline & QUALITY WORK-LIFE & .976 & $p=0$ \\
\hline & Loyalty Score & .984 & $p=0$ \\
\hline Passive & SUPERVISOR & & \\
\hline & COMPANY & .943 & $p=0$ \\
\hline & OPPORTUNITY & .961 & $p=0$ \\
\hline & QUALITY WORK-LIFE & .840 & $p=0$ \\
\hline & Loyalty Score & .978 & $p=0$ \\
\hline & & $p=0$ \\
\hline SUP-seeker & SUPEVISOR & .881 & $p=0$ \\
\hline & COMPANY & .886 & $p=0$ \\
\hline OPPORTUNITY & .821 & $p=0$ \\
\hline & QUALITY WORK-LIFE & .981 & $P=.161$ \\
\hline Loyalty Score & .929 & $P=.0001$ \\
\hline
\end{tabular}

Table 2.3.2 Shapiro-Wilk's test for factor and loyalty normality by seeking status 


\section{Seeker}

Graphic normality checks further support the rejection of the null of normality for seekers based on the distributions of each of the factors.

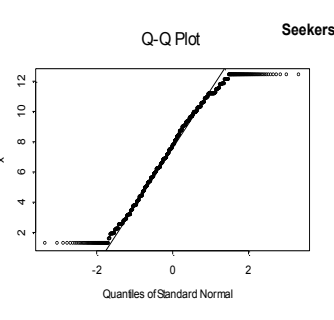

Histogram

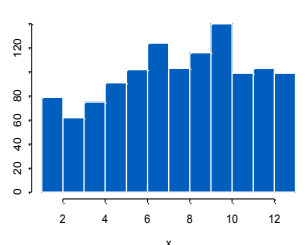

Figure 2.3.1 Seekers - Dist. not normal SUPV
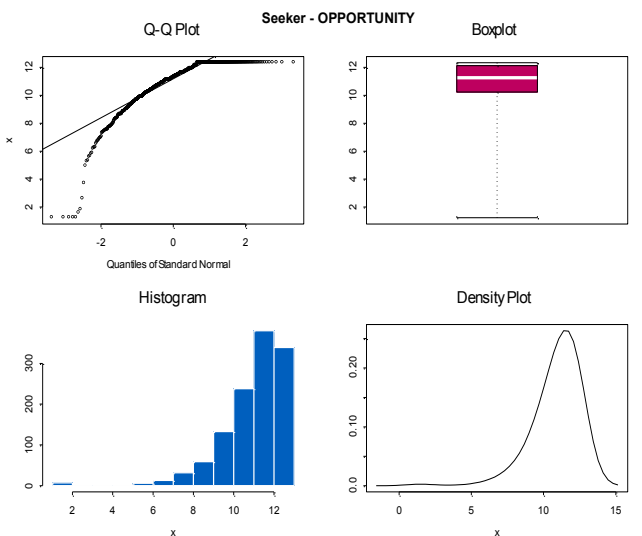

Figure 2.3.3 Seekers - Dist. not normal OPPTY
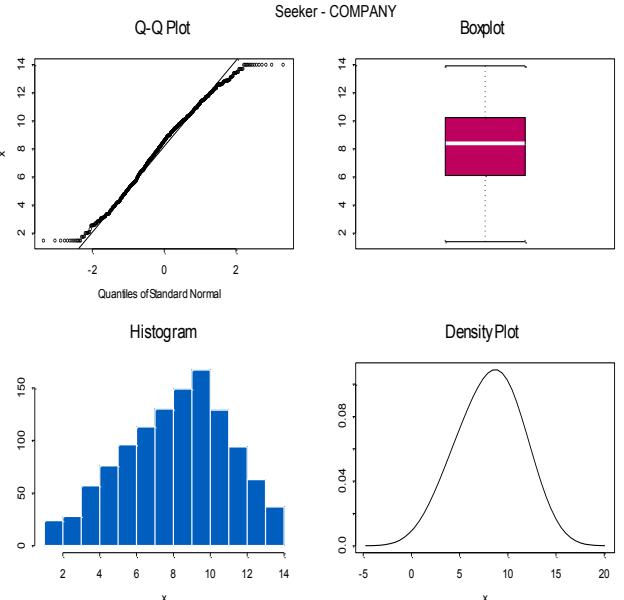

Figure 2.3.2 Seekers - Dist. not normal COMP
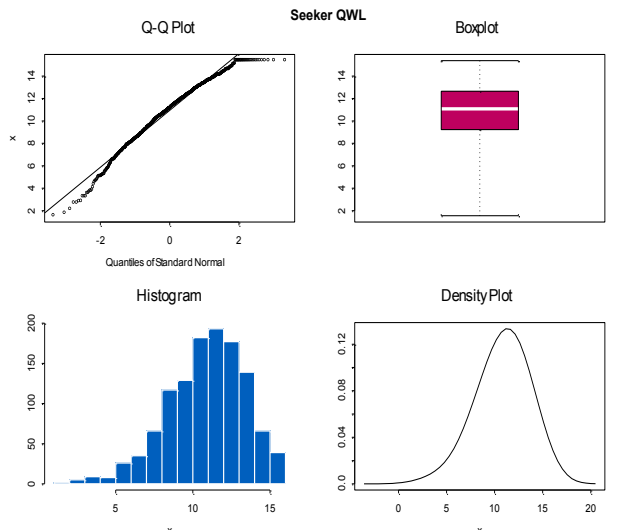

Figure 2.3.4 Seekers - Dist. not normal QWL

\section{Seekers - Loyalty Score}

Scores for seekers' loyalty range from -157.95 to 457.81 , with a mean score of 186.71. There is substantial deviation with $\sigma=20.33$. The Q-Q plot evidences heavy tails, and skewness, $\gamma=338$, and kurtosis $\gamma=-185$. The loyalty score for seekers is not normally distributed. 

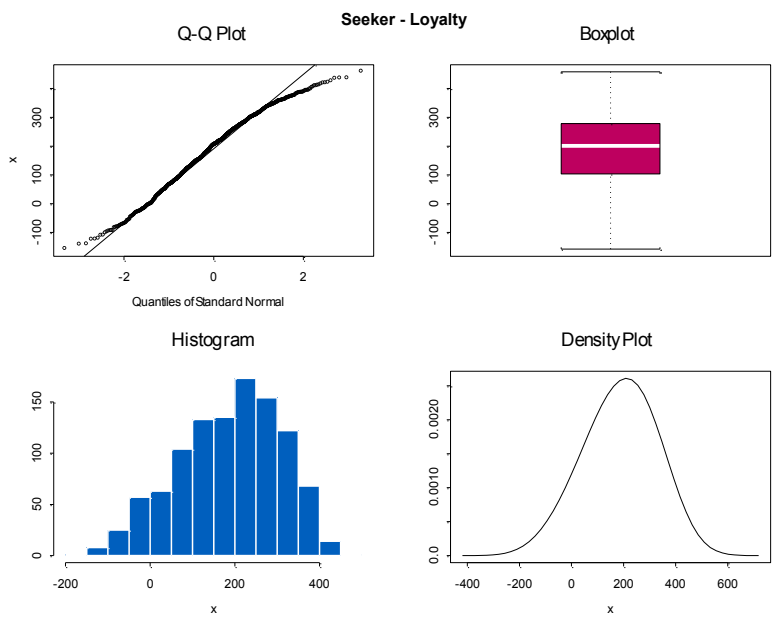

Figure 2.3.5 Seeker-Loyalty score not normally distributed

Table 2.3.3 and Table 2.3.4 summarize the descriptive statistics discussed in this section on seekers and their distributions on the factors and the loyalty score.

\begin{tabular}{|l|l|l|l|l|l|l|l|}
\hline Seekers - Descriptive Statistics & $\mathrm{N}$ & Range & Min & Max & Mean & $\begin{array}{l}\text { Std. } \\
\text { Deviation }\end{array}$ \\
\hline & Statistic & Statistic & Statistic & Statistic & Statistic & $\begin{array}{l}\text { Std. } \\
\text { Error }\end{array}$ & Statistic \\
\hline SUPERVISOR & 1193 & 11.14 & 1.24 & 12.38 & 7.40 & .093 & 3.24 \\
\hline COMPANY & 1163 & 12.51 & 1.39 & 13.90 & 8.13 & .084 & 2.87 \\
\hline OPPORTUNITY & 1210 & 11.10 & 1.23 & 12.33 & 10.91 & .045 & 1.57 \\
\hline $\begin{array}{l}\text { QUALITY WORK } \\
\text { LIFE }\end{array}$ & 1193 & 13.83 & 1.54 & 15.37 & 10.84 & .072 & 2.51 \\
\hline \begin{tabular}{l} 
LOYALTY SCORE \\
\hline
\end{tabular} & 1058 & 615.77 & -157.95 & 457.81 & 186.71 & 3.7 & 120.33 \\
\hline
\end{tabular}

Table 2.3.3

Descriptive statistics for seekers

\begin{tabular}{|l|l|l|l|l|l|}
\hline \multicolumn{4}{|l|}{ Seeker - Descriptive Statistics } & \multicolumn{4}{l|}{ Kurtosis } \\
\hline & Statistic & Statistic & Std. Error & Statistic & Std. Error \\
\hline SUPERVISOR & 1193 & -.230 & .071 & -.992 & .142 \\
\hline COMPANY & 1163 & -.228 & .072 & -.616 & .143 \\
\hline OPPORTUNITY & 1210 & -2.23 & .070 & 8.44 & .141 \\
\hline QUALITY WORK LIFE & 1193 & -.592 & .071 & .348 & .142 \\
\hline LOYALTY SCORE & 1058 & .338 & .075 & -.485 & .15 \\
\hline
\end{tabular}

Table 2.3.4 Skewness and kurtosis for seekers suggest non-Gaussian distributions 
Passives - Descriptive Statistics

Next consider just the passive seekers, those who would consider a new job given the right opportunity ( $\mathrm{n}=467,25.7 \%$ of the dataset), This group is significantly different from those who report themselves as seekers on all factors with the exception of OPPORTUNITY. Passives are significantly different from non-seekers on all factors with the exception of QUALITY of WORK-LIFE. Table 2.3.8 through Table 2.3.13 provide details on significant differences between subclasses with regard to the factors and loyalty score.

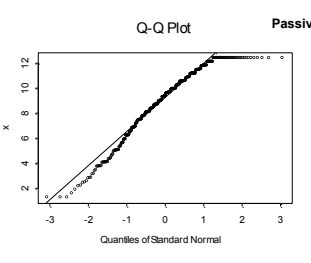

Histogram

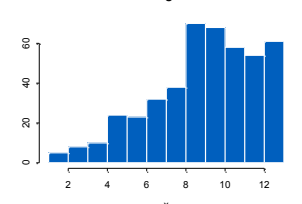

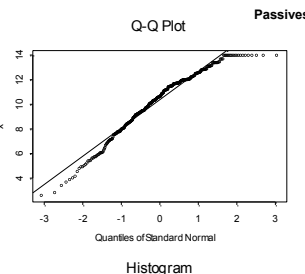
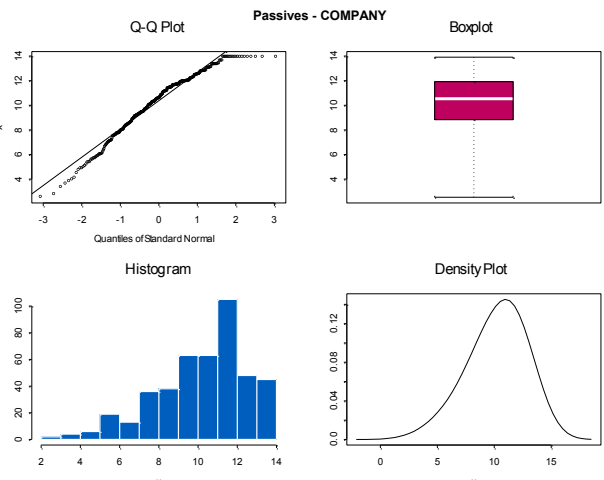

Figure 2.3.6 Passives - Dist. not normal SUPV
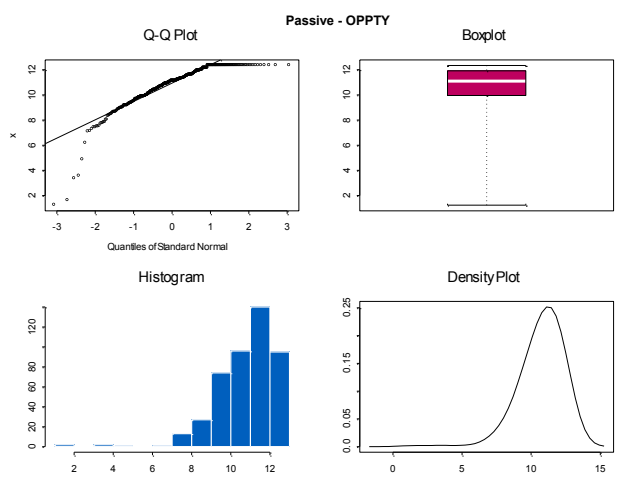

Figure 2.3.8 Passives - Dist. not normal OPPTY
Figure 2.3.7 Passives - Dist. not normal COMP
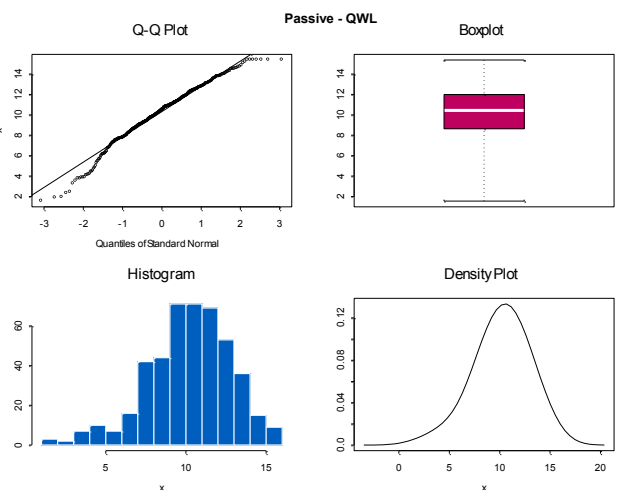

Figure 2.3.9 Passives - Dist. not normal - QWL 
Passives - Loyalty Score

The loyalty score for passives ranges from a minimum of -76.38 to a maximum of 504.99 , with $\mu=274.22$ and $\sigma=98.79$. The score is significantly higher than that for seekers, where $\mu=186.71$, and significantly lower than non-seekers at $\mu=343.94$, differentiating the subclasses. Skewness is left again, with $\gamma=-; 9$ and kurtosis at $\gamma=161$. The heavier left tail is seen in the density and Q-Q plots.
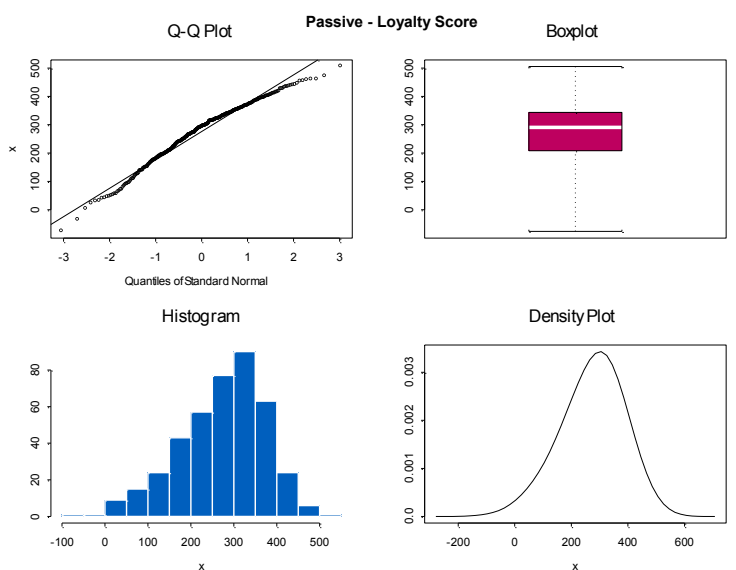

Figure 2.3.10 Passives - Loyalty score not normally distributed

Following, Table 2.3.5 and Table 2.3.6 summarize the descriptive statistics for passives discussed in this section.

\begin{tabular}{|l|l|l|l|l|l|l|l|}
\hline Passive - Descriptive Statistics & Man & Range & Min & Max & $\begin{array}{l}\text { Std. } \\
\text { Dev. }\end{array}$ \\
\hline & Statistic & Statistic & Statistic & Statistic & Statistic & Std. Error & Statistic \\
\hline SUPERVISOR & 451 & 11.14 & 1.24 & 12.38 & 8.9012 & .125 & 2.65 \\
\hline COMPANY & 442 & 11.38 & 2.52 & 13.90 & 10.213 & .111 & 2.34 \\
\hline OPPORTUNITY & 451 & 11.10 & 1.23 & 12.33 & 10.749 & .070 & 1.5 \\
\hline $\begin{array}{l}\text { QUALITY WORK } \\
\text { LIFE }\end{array}$ & 455 & 13.83 & 1.54 & 15.37 & 10.184 & .122 & 2.61 \\
\hline $\begin{array}{l}\text { LOYALTY } \\
\text { SCORE }\end{array}$ & 411 & 581.37 & -76.38 & 504.99 & 274.22 & 4.87 & 98.78 \\
\hline
\end{tabular}

Table 2.3.5 Descriptive statistics for passives 


\begin{tabular}{|l|l|l|l|l|l|}
\hline \multicolumn{9}{|l|}{ Passives - Descriptive Statistics } & $\mathrm{N}$ & \multicolumn{4}{l|}{ Skewness } & \multicolumn{2}{l|}{ Kurtosis } \\
\hline & Statistic & Statistic & Std. Error & Statistic & Std. Error \\
\hline SUPERVISOR & 451 & -.695 & .115 & -.164 & .23 \\
\hline COMPANY & 442 & -.672 & .116 & .078 & .23 \\
\hline OPPORTUNITY & 451 & -2.05 & .115 & 8.17 & .22 \\
\hline QUALITY WORK LIFE & 455 & -.565 & .114 & .450 & .22 \\
\hline LOYALTY SCORE & 411 & -.590 & .120 & .161 & .24 \\
\hline
\end{tabular}

Table 2.3.6 Skewness and kurtosis for passives

Non-seeker Descriptive Statistics

The final subclass investigated is the non-seeker group. These people identified themselves as not currently seeking a new job, staying in their current position a while. There are 104 non-seekers, representing 5.7\% of the dataset of respondents, employed full-time at the time of the survey. The nonseekers significantly differ from the seekers on all factors and the loyalty score. They also differ from passives on all factors with the exception of the influence of the variables comprising QUALITY of WORK-LIFE. There is substantial variation within the class which reflects non-normal distributions with regard to the factors.
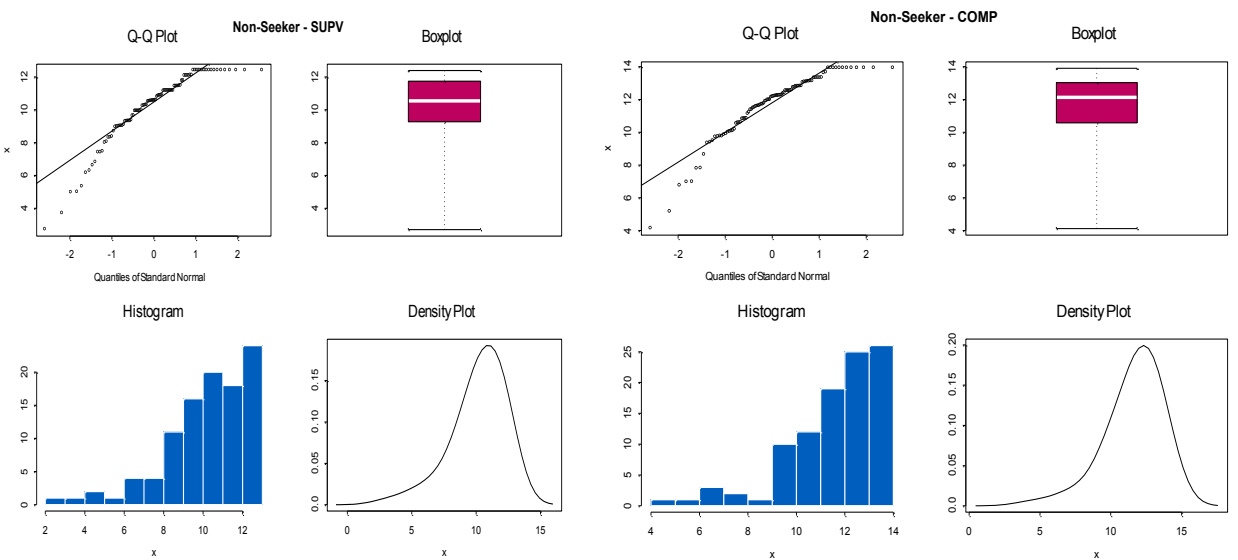

Figure 2.3.11 Non-seekers - Dist. not normal - SUPV

Figure 2.3.12 Non-seekers not normal -COMP 

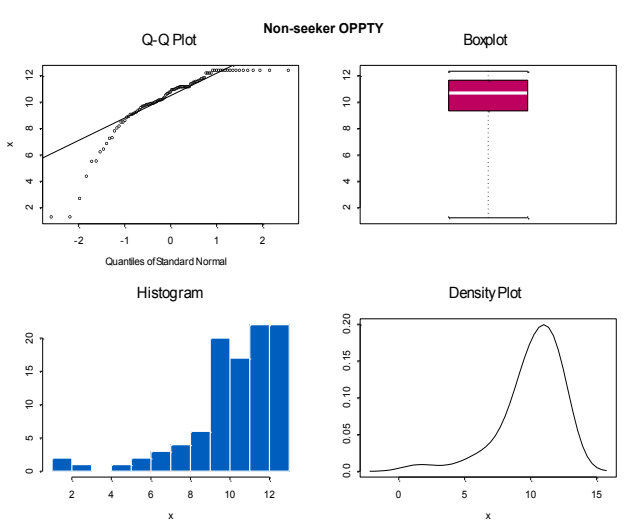

Figure 2.3.13 Non-seekers dist. not normal -OPPTY

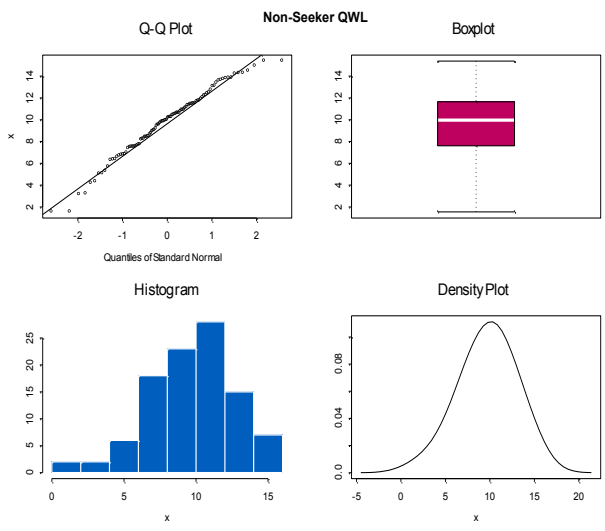

Figure 2.3.14 Non-seekers not normal - QWL

\section{Non-seeker LOYALTY SCORE}

Consider non-seeker scores for the loyalty measurement. Ranging from 11.20 to 557.14 , there is a 568.34 spread. The mean loyalty score of 343.94 is significantly higher than both passives at 274.22 and seekers at 186.71. The standard deviation decreases accordingly to 80.97, whereas it was larger for passives at 98.78 and even larger for seekers at 120.33. This suggests that employees who are more loyal to their organization have less diversity in their perceptions of supervisor, company, opportunity and quality of work life, as compared to passives and seekers. The skewness statistic $\gamma_{-}=-i 83$ indicating a left skew, and kurtosis is positive, leptokurtic, at $\gamma=554$. These statistics indicating shape are clarified by the density plot which reflects the slight bump in the fat left tail (Figure 2.3.15).

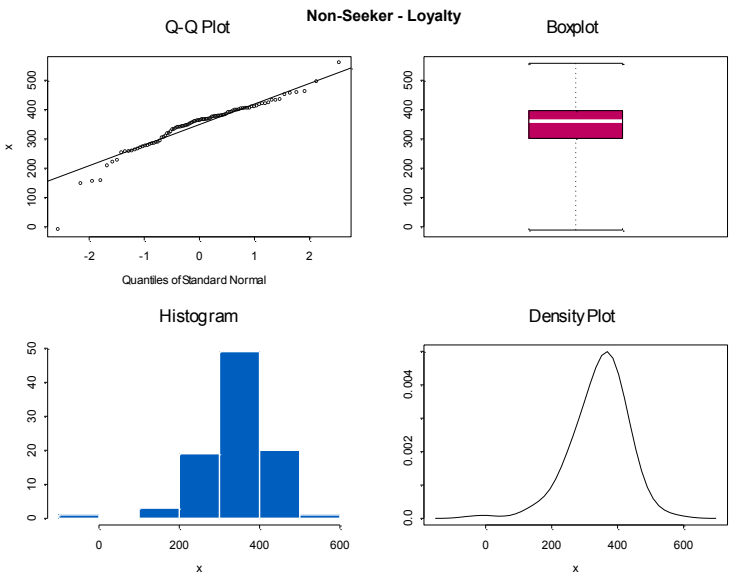

Figure 2.3.15 Loyalty scores for non-seekers are not normally distributed

The following tables (Table 2.3.7 and Table 2.3.8), provide descriptive statistics for non-seekers on the four factors and the loyalty score 


\begin{tabular}{|l|l|l|l|l|l|l|l|}
\hline Non-Seeker - Descriptive Statistics \\
& $\mathrm{N}$ & Range & Min & Max & Mean & $\begin{array}{l}\text { Std. } \\
\text { Deviation }\end{array}$ \\
\hline & Statistic & Statistic & Statistic & Statistic & Statistic & $\begin{array}{l}\text { Std. } \\
\text { Error }\end{array}$ & Statistic \\
\hline SUPERVISOR & 102 & 9.68 & 2.70 & 12.38 & 10.15 & .20 & 2.06 \\
\hline COMPANY & 100 & 9.78 & 4.12 & 13.90 & 11.60 & .19 & 1.95 \\
\hline OPPORTUNITY & 100 & 11.10 & 1.23 & 12.33 & 10.09 & .23 & 2.30 \\
\hline $\begin{array}{l}\text { QUALITY WORK } \\
\text { LIFE }\end{array}$ & 101 & 13.83 & 1.54 & 15.37 & 9.710 & .29 & 3.01 \\
\hline $\begin{array}{l}\text { LOYALTY SCORE } \\
\text { OOY }\end{array}$ & 93 & 568.34 & -11.20 & 557.14 & 343.94 & 8.40 & 80.97 \\
\hline
\end{tabular}

Table 2.3.7 Descriptive statistics non-seekers

\begin{tabular}{|l|l|l|l|l|l|}
\hline Non-seeker Descriptive Statistics \\
& N & \multicolumn{2}{l|}{ Skewness } & \multicolumn{2}{l|}{ Kurtosis } \\
\hline & Statistic & Statistic & Std. Error & Statistic & Std. Error \\
\hline SUPERVISOR & 102 & -1.31 & .239 & 1.84 & .474 \\
\hline COMPANY & 100 & -1.39 & .241 & 2.43 & .478 \\
\hline OPPORTUNITY & 100 & -1.83 & .241 & 4.12 & .478 \\
\hline QUALITY WORK LIFE & 101 & -.422 & .240 & .018 & .476 \\
\hline LOYALTY SCORE & 93 & -1.14 & .250 & 3.64 & .495 \\
\hline
\end{tabular}

Table 2.3.8 Skewness and kurtosis for non-seekers

Significant Differences Between Subclasses on Factors and Loyalty

Given the factors developed to reduce dimensionality, it is valuable to briefly summarize the areas in which significant differences between the subclasses are evident. Specifically, seekers and non-seekers evidence statistically significant differences on all factors, as well as the loyalty score. Seekers and passives differ significantly on all factors with the exception of OPPORTUNITY. Lastly, the passives differ from the non-seekers on all factors with the exception of QUALITY of WORK-LIFE (see Table 2.3.9 through Table 2.3.12). Table 2.3.13 compares loyalty scores by seeking status.

\begin{tabular}{|c|c|c|c|c|c|c|}
\hline $\begin{array}{l}\text { SUPERVISOR } \\
\text { FACTOR }\end{array}$ & Mean & T Stat & $\begin{array}{l}\text { Degrees } \\
\text { freedom }\end{array}$ & $\begin{array}{l}\mathrm{p}- \\
\text { value }\end{array}$ & $\begin{array}{l}\text { Mean } \\
\text { diff }\end{array}$ & $\begin{array}{l}\text { Std. } \\
\text { error } \\
\text { diff }\end{array}$ \\
\hline Seekers & 7.40 & \multirow[t]{2}{*}{-12.27} & \multirow[t]{2}{*}{147} & \multirow[t]{2}{*}{.000} & \multirow[t]{2}{*}{-2.75} & \multirow[t]{2}{*}{.22} \\
\hline Non-seekers & 10.16 & & & & & \\
\hline Seekers & 7.40 & \multirow[t]{2}{*}{-9.59} & \multirow[t]{2}{*}{981} & \multirow[t]{2}{*}{.000} & \multirow[t]{2}{*}{-1.50} & \multirow[t]{2}{*}{.15} \\
\hline Passives & 8.90 & & & & & \\
\hline Passives & 8.90 & \multirow[t]{2}{*}{-5.25} & \multirow[t]{2}{*}{185} & \multirow[t]{2}{*}{.000} & \multirow[t]{2}{*}{-1.25} & \multirow[t]{2}{*}{.23} \\
\hline Non-seekers & 10.16 & & & & & \\
\hline
\end{tabular}




\begin{tabular}{|c|c|c|c|c|c|c|}
\hline COMPANY FACTOR & Mean & T Stat & $\begin{array}{l}\text { Degrees } \\
\text { freedom }\end{array}$ & $\begin{array}{l}\mathrm{p}- \\
\text { value }\end{array}$ & Mean diff & $\begin{array}{l}\text { Std. } \\
\text { error } \\
\text { diff }\end{array}$ \\
\hline Seekers & 8.13 & \multirow[t]{2}{*}{-16.29} & \multirow[t]{2}{*}{138} & \multirow[t]{2}{*}{.000} & \multirow[t]{2}{*}{-3.46} & \multirow[t]{2}{*}{.21} \\
\hline Non-seekers & 11.60 & & & & & \\
\hline Seekers & 8.13 & \multirow[t]{2}{*}{-14.85} & \multirow[t]{2}{*}{968} & \multirow[t]{2}{*}{.000} & \multirow[t]{2}{*}{-2.07} & \multirow[t]{2}{*}{.13} \\
\hline Passives & 10.21 & & & & & \\
\hline Passives & 10.21 & \multirow[t]{2}{*}{-6.16} & \multirow[t]{2}{*}{170} & \multirow[t]{2}{*}{.000} & \multirow[t]{2}{*}{-1.38} & \multirow[t]{2}{*}{.22} \\
\hline Non-seekers & 11.60 & & & & & \\
\hline
\end{tabular}

Table 2.3.10 All subclasses differ significantly on the COMP factor

\begin{tabular}{|c|c|c|c|c|c|c|}
\hline $\begin{array}{l}\text { OPPORTUNITY } \\
\text { FACTOR }\end{array}$ & Mean & T Stat & $\begin{array}{l}\text { Degrees } \\
\text { freedom }\end{array}$ & $\begin{array}{l}\mathrm{p}- \\
\text { value }\end{array}$ & $\begin{array}{l}\text { Mean } \\
\text { diff }\end{array}$ & $\begin{array}{l}\text { Std. } \\
\text { error } \\
\text { diff }\end{array}$ \\
\hline Seekers & 10.91 & \multirow[t]{2}{*}{3.48} & \multirow[t]{2}{*}{106} & \multirow[t]{2}{*}{.000} & \multirow[t]{2}{*}{.81} & \multirow[t]{2}{*}{.234} \\
\hline Non-seekers & 10.09 & & & & & \\
\hline Seekers & 10.91 & \multirow{2}{*}{$\begin{array}{l}\text { Not } \\
\text { Significant }\end{array}$} & & & & \\
\hline Passives & 10.74 & & & & & \\
\hline Passives & 10.74 & \multirow[t]{2}{*}{2.71} & \multirow[t]{2}{*}{118} & \multirow[t]{2}{*}{.000} & \multirow[t]{2}{*}{.65} & \multirow[t]{2}{*}{.240} \\
\hline Non-seekers & 10.09 & & & & & \\
\hline
\end{tabular}

Table 2.3.11 No significant difference between passives and seekers on OPPTY factor

\begin{tabular}{|c|c|c|c|c|c|c|}
\hline $\begin{array}{l}\text { QUALTITY of } \\
\text { WORK LIFE } \\
\text { FACTOR }\end{array}$ & Mean & T Stat & $\begin{array}{l}\text { Degrees } \\
\text { freedom }\end{array}$ & $\begin{array}{l}\mathrm{p}- \\
\text { value }\end{array}$ & $\begin{array}{l}\text { Mean } \\
\text { diff }\end{array}$ & $\begin{array}{l}\text { Std. } \\
\text { error } \\
\text { diff }\end{array}$ \\
\hline Seekers & 10.84 & \multirow[t]{2}{*}{3.66} & \multirow[t]{2}{*}{112} & \multirow[t]{2}{*}{.000} & \multirow[t]{2}{*}{1.12} & \multirow[t]{2}{*}{.30} \\
\hline Non-seekers & 9.71 & & & & & \\
\hline Seekers & 10.84 & \multirow[t]{2}{*}{4.68} & \multirow[t]{2}{*}{1646} & \multirow[t]{2}{*}{.000} & \multirow[t]{2}{*}{.65} & \multirow[t]{2}{*}{.14} \\
\hline Passives & 10.18 & & & & & \\
\hline Passives & 10.18 & \multirow{2}{*}{$\begin{array}{l}\text { Not } \\
\text { Significant }\end{array}$} & & & & \\
\hline Non-seekers & 9.71 & & & & & \\
\hline
\end{tabular}

\begin{tabular}{|c|c|c|c|c|c|c|}
\hline $\begin{array}{l}\text { LOYALTY } \\
\text { SCORE }\end{array}$ & Mean & T Stat & $\begin{array}{l}\text { Degrees } \\
\text { freedom }\end{array}$ & $\begin{array}{l}\mathrm{p}- \\
\text { value }\end{array}$ & Mean diff & $\begin{array}{l}\text { Std. } \\
\text { error } \\
\text { diff }\end{array}$ \\
\hline Seekers & 186.71 & -17.14 & 131 & .000 & -157.23 & 9.17 \\
\hline Non-seekers & 343.94 & & & & & \\
\hline Seekers & 186.71 & -14.3 & 908 & .000 & -87.51 & 6.12 \\
\hline Passives & 274.22 & & & & & \\
\hline Passives & 274.22 & -7.18 & 160 & .000 & -69.71 & 9.71 \\
\hline Non-seekers & 343.94 & & & & & \\
\hline
\end{tabular}

Table 2.3.13 Significant differences on loyalty score for all subclasses 


\section{Parallel Boxplots}

This section ends with a comparison of the subclasses utilizing parallel boxplots. The idea is to compare the three subclasses of seekers, passives and non-seekers simultaneously, graphically. Visualizing these often subtle differences clarifies the descriptive statistics surrounding each of the factors and ultimately, the loyalty score.

Outliers are represented by open circles as in the following parallel boxplots. Points lying more than 3 box lengths from either end of the box are extreme outliers marked with an asterisk.

Begin with a comparative look at the SUPERVISOR factor for each of the subclasses (Figure 2.3.16).

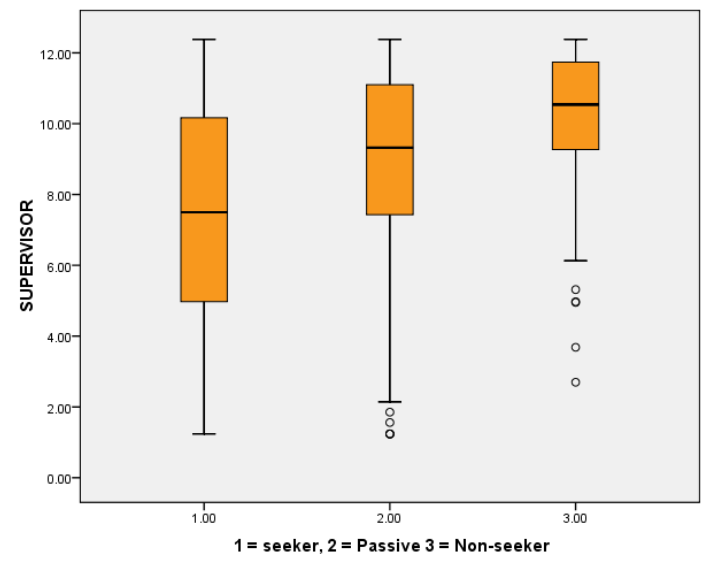

Figure 2.3.16 - Parallel boxplots of seeking status on supervisor factor

Seekers appear to be the most normally distributed of the three subclasses, yet skewed left as indicated by the longer lower whisker, yet with more variability as indicated by the interquartile range, or length of the box itself. Passives on the other hand have a more serious left skew as evidenced by the lower whisker much longer than the box. Three outliers are present in the subclass, and fall rather close to the minimum, thus not investigated further or omitted. Non-seeker scores for SUPERVISOR evidence the variability among the classes, yet with the most pronounced left skew.

Next, in Figure 2.3.17, the COMPANY factor reflects a similar pattern among the subclasses, with increasing ratings and decreasing variability. A few more outliers are present in the passive data for COMPANY than seen for SUPERVISOR but one less for non-seekers. Non-seekers evidence a median closer to the upper quartile. 


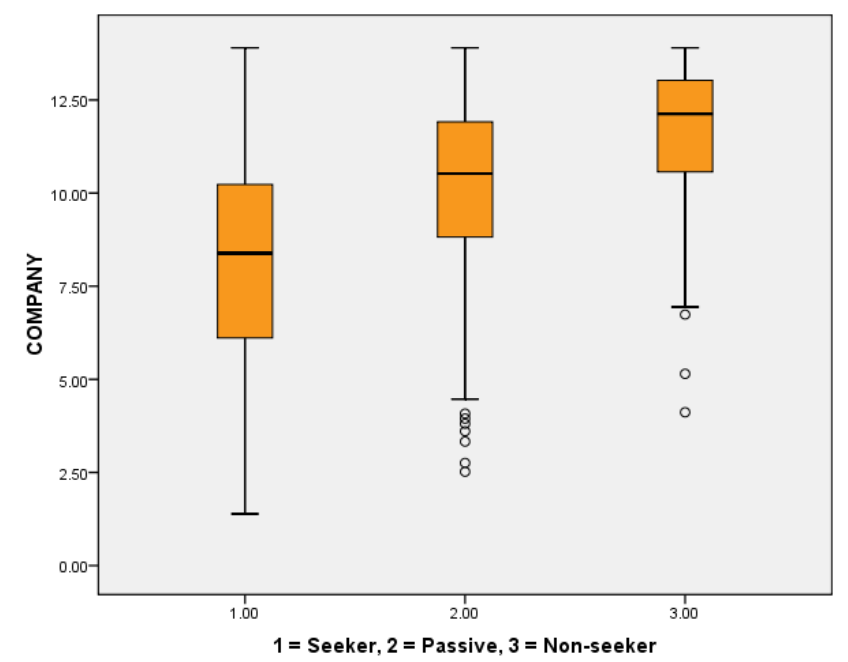

Figure 2.3.17 - Parallel boxplots of seeking status on company factor

The OPPORTUNITY factor, presented in parallel boxplots for subclasses in Figure 2.3.18 helps with visualizing the commonalities between all respondents regarding the influence of the potential for a better opportunity elsewhere. One can ascertain that all respondents are highly influenced by the potential for higher salary, better benefits, greater advancement opportunity and better skills training which is to be expected to a certain degree. It is interesting to note the very similar extent of variability for seekers and passives as evidenced by the similar lengths of the boxplots, (recall $\sigma_{\text {.eker }}=.572$ and $\sigma_{\text {r zssive }}=.503$ ), compared to the increased variability of the non-seeker with regard to the influence of potential OPPORTUNITY, where $\sigma_{\text {,nseeker }}=$ !.301.

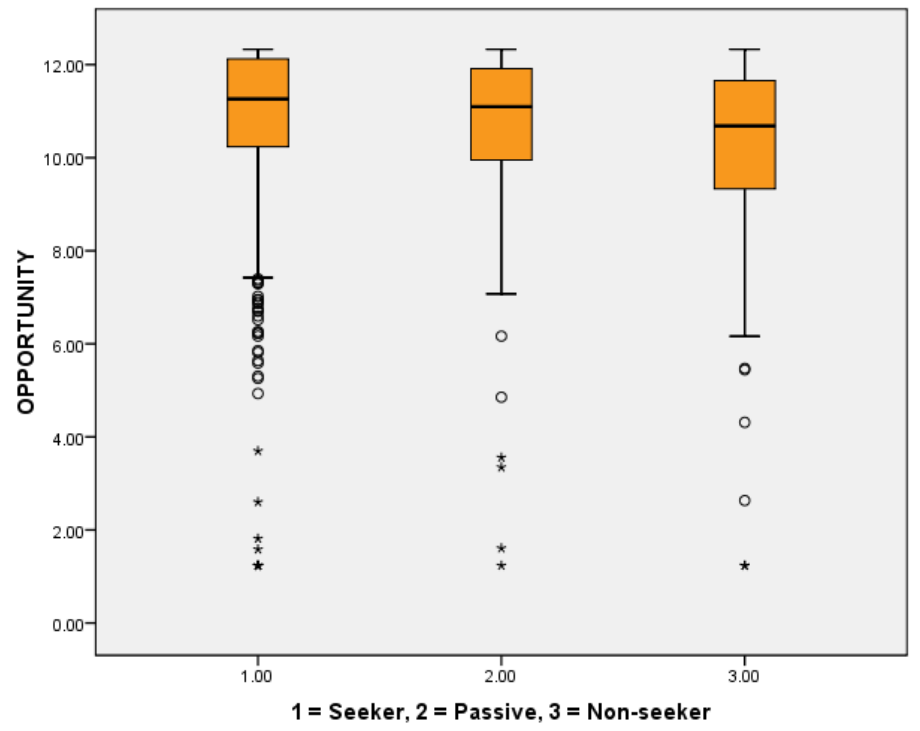

Figure 2.3.18 - Parallel boxplots of seeking status on opportunity factor 
Moving onto the QUALITY of WORK-LIFE factor (Figure 2.3.19), this is perhaps the most normal of all the factors, as evidenced by the relative symmetry of the boxplots for all subclasses.. Seekers do evidence more of a left skew and sustain more outliers, whereas non-seekers are much closer to being normally distributed with only one outlier.

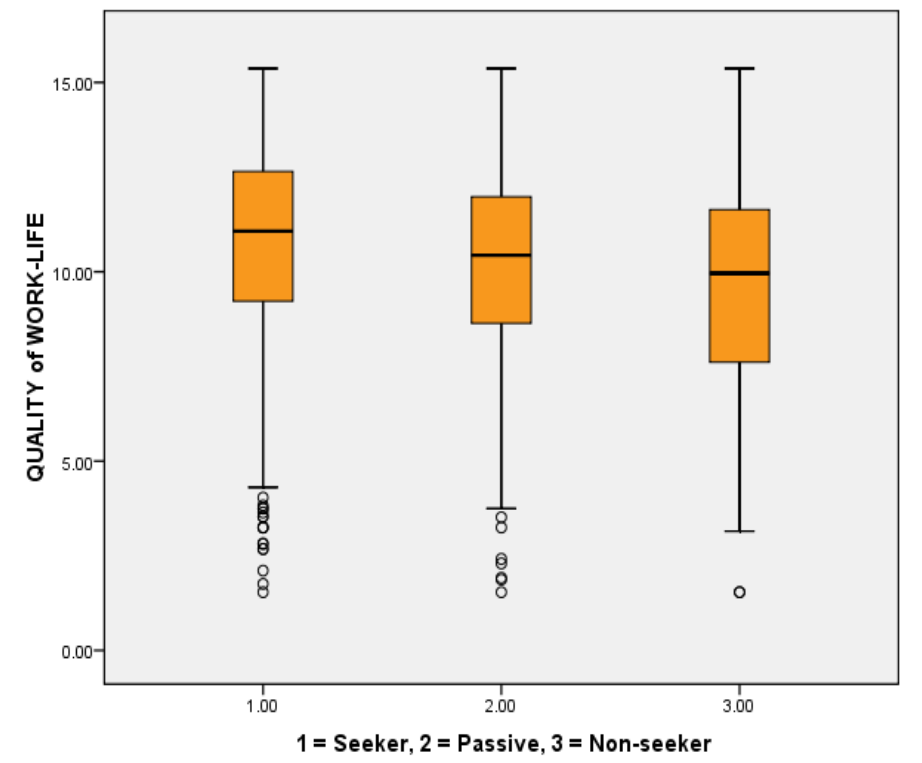

Figure 2.3.19 - Parallel boxplots of seeking status on quality of work-life factor

Lastly, the loyalty score is reviewed (Figure 2.3.20). Although there are still several outliers, it is not likely that their impact is substantial given that there are $n=1246$ seekers with fewer than 10 outliers, $n=467$ with one extreme passive outlier, and $n=104$ with fewer than 5 non-seeking outliers. Variability is fairly consistent among the three subclasses and the lengths of the whiskers, relative to the boxplots, are acceptably balanced. 


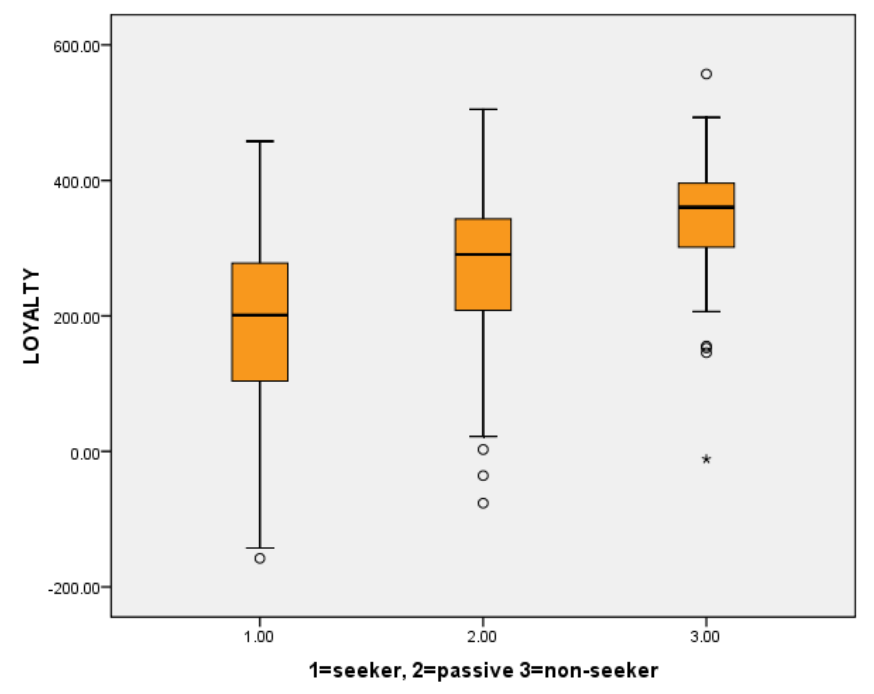

Figure 2.3.20 - Parallel boxplots of seeking status on loyalty score

Summary of Part 2 - Section 2.1 through Section 2.3

In Section 2.1 the loyalty score was developed and its components were discussed. An estimation process was detailed, and an application was made using the job-seeker dataset. In Section 2.2, the descriptive statistics for the loyalty score and its components were presented and evaluated. Both statistical and graphical checks for normality of the factors and the loyalty score suggested that generally, the data is not normally distributed. Section 2.3 focused on the subclasses, or seeking categories. The descriptive statistics for the loyalty score and its components by subclass were investigated and the distributions by subclasses were not reflective of Gaussian data.

The motivation for this portion of the analysis was not only to defend the method used to develop the loyalty score, but also to better understand the underlying factors and how they potentially differ by seeking status. It is clear from Section 2.3 that significant differences exist between the seekers, passives and non-seekers with regard to supervisor and company satisfaction as well as the influence of alternatives that may yield better opportunities or quality of work-life.

The loyalty score enabled the identification of seekers and non-seekers with $75 \%$ accuracy, based on the job-seeker dataset, but the full information, particularly that given by the passives, may not be utilized completely.

Upcoming sections will discuss alternate methods of identifying seeking status based on a dataset of job satisfaction and outside influence attitudes and behaviors. 


\subsection{Conditional Probability Given Loyalty Score}

Reasoning and Goal

This section considers the conditional probability of seeking status given the loyalty score. The aim is to find the empirical probability that person $x$, given the loyalty score, falls into category $y$.

Consider, if $A$ and $B$ are events in $S$, and $\mathrm{P}(B)>0$, then the conditional probability of $A$ given $B$, written $\mathrm{P}(A \mid B)$, is

$$
P(A \mid B)=\frac{P(A \cap)}{P(B)}
$$

In the conditional probability calculation, $B$ becomes the sample space $\mathrm{P}(B \mid B)$ $=1$, and the original sample space $S$ is updated to $B$. Also consider the conditional probabilities of disjoint sets, where if $A$ and $B$ are disjoint, $P(A \cap B)=$ ). It then follows that $P(A \mid B)=P(B \mid A)=$ ) (Casella \& Berger, p.20).

Consider the sample space $B$ to be the scientific loyalty scores, and $\mathrm{P}(A)$ to be the empirical probability that, given the loyalty score, a person $x$ falls in to category $y$.

As a starting point, visual binning is used to categorize the loyalty scores. Visual binning is designed to assist in the process of creating new variables based on grouping contiguous values of existing variables into a limited number of distinct categories.

Application to Job Seeker Data

The loyalty scores are categorized into nine equally-sized bins, based on intervals of the scores. The quantity of nine bins is selected because this is a manageable number of score classifications for an employer (intuitively allowing for three levels per seeking category).

In Table 2.4.1 the exact interval is reported, the $n$ respondents who fall into the interval, the $n$ in each seeking category within the interval, and the percentage correctly classified. For example, 146 seekers were identified in Bin 1 . Dividing this number by the total number of seekers (146/1058), we can say that $14 \%$ of the seekers were correctly classified into Bin 1, based on their loyalty scores between -157.95 and 46.97 . 


\begin{tabular}{|c|c|c|c|c|c|c|c|c|c|}
\hline Bin & 1 & 2 & 3 & 4 & 5 & 6 & 7 & 8 & 9 \\
\hline Score & $\begin{array}{l}-157.95 \\
\text { to } \\
46.97\end{array}$ & $\begin{array}{l}46.98 \\
\text { to } \\
110.43\end{array}$ & $\begin{array}{l}110.93 \\
\text { to } \\
156.47\end{array}$ & $\begin{array}{l}156.5 \\
\text { to } \\
200.71\end{array}$ & $\begin{array}{l}200.84 \\
\text { to } \\
233.46\end{array}$ & $\begin{array}{l}233.99 \\
\text { to } \\
266.74\end{array}$ & $\begin{array}{l}267.01 \\
\text { to } \\
298.3\end{array}$ & $\begin{array}{l}298.57 \\
\text { to } \\
332.22\end{array}$ & $\begin{array}{l}332.37 \\
\text { to } \\
366.79\end{array}$ \\
\hline$n$ & 157 & 155 & 157 & 156 & 156 & 157 & 156 & 156 & 156 \\
\hline & $\begin{array}{c}\text { \% of } \\
\text { category } \\
\text { in bin }\end{array}$ & & & & & & & & \\
\hline Seeker & $14 \%$ & $13 \%$ & $13 \%$ & $11 \%$ & $12 \%$ & $10 \%$ & $9 \%$ & $8 \%$ & $7 \%$ \\
\hline Passive & $2 \%$ & $5 \%$ & $5 \%$ & $10 \%$ & $8 \%$ & $11 \%$ & $13 \%$ & $15 \%$ & $15 \%$ \\
\hline $\begin{array}{l}\text { Non- } \\
\text { Seeker }\end{array}$ & $1 \%$ & & $3 \%$ & & $3 \%$ & $6 \%$ & $11 \%$ & $9 \%$ & $25 \%$ \\
\hline
\end{tabular}

Table 2.4.1 Percentage correctly classified into each interval

The method correctly classifies $51 \%$ of the seekers with scores less than or equal to 200.71. Non-seekers are found at the higher end of the ranked scores, with $54 \%$ of them scoring 200.84 or higher. Fifty-four percent of the passives had scores higher than 233.99 .

Breaking the entire sample into nine equal intervals is not a particularly compelling method for identifying seeking status.

Comparative Sample with Equal Subclasses, Three Bins

Consider that the dataset is comprised primarily of seekers, nearly $70 \%$, thus it makes sense to level the playing field by selecting equally sized subclasses for comparison. There are only 93 non-seekers with no missing data points for the loyalty score, so all are used. Out of 411 passives, 93 are randomly selected, and 93 seekers are randomly selected out of 1246. The number of bins is reduced to a more manageable three.

The first bin, comprised of scores from -124.83 to 247.73 identifies nearly $60 \%$ of the seekers in the sample. The second bin reveals $38 \%$ of the passives with scores between 247.84 and 340.35, comparable to Bin 6 through Bin 8 when all data were used (see Table 2.4.1). Sixty-two percent of the nonseekers were correctly classified into Bin 3 when equal sized subclasses were used, with scores higher than 340.35. Table 2.4.2 summarizes the results for this sample. 


\begin{tabular}{|c|c|c|c|}
\hline Bin & 1 & 2 & 3 \\
\hline Score & $\begin{array}{l}-124.83 \\
\text { to } \\
247.73\end{array}$ & $\begin{array}{l}247.84 \\
\text { to } \\
340.35\end{array}$ & $\begin{array}{l}340.35 \\
\text { to } \\
557.14\end{array}$ \\
\hline$N$ & 93 & 93 & 93 \\
\hline & $\begin{array}{c}\% \text { of category in } \\
\text { bin }\end{array}$ & & \\
\hline Seeker & $59 \%$ & $31 \%$ & $10 \%$ \\
\hline Passive & $33 \%$ & $38 \%$ & $28 \%$ \\
\hline Non-Seeker & $8 \%$ & $31 \%$ & $62 \%$ \\
\hline
\end{tabular}

Table 2.4.2 Percentage correctly classified - Sample with equal subclasses

Three Bins - all data

Next investigated is the three bin approach with all the data, calling for evenly distributed groups. The most accurately identified are the non-seekers, $76 \%$ of which are classified into Bin 3 with loyalty scores greater than 340.35. Bin 2 was not very promising, finding only a third of the passives, yet as good as chance alone. Bin 1 captured $43 \%$ of the seekers with scores less than or equal to 173.24 .

\begin{tabular}{|c|c|c|c|}
\hline Bin & 1 & 2 & 3 \\
\hline Score & $\begin{array}{l}-157.95 \\
\text { to } \\
173.24\end{array}$ & $\begin{array}{l}247.84 \\
\text { to } \\
340.35\end{array}$ & $\begin{array}{l}340.35 \\
\text { to } \\
557.14\end{array}$ \\
\hline \multirow[t]{2}{*}{$N$} & 521 & 521 & 520 \\
\hline & $\begin{array}{c}\% \text { of category in } \\
\text { bin }\end{array}$ & & \\
\hline Seeker & $43 \%$ & $35 \%$ & $22 \%$ \\
\hline Passive & $15 \%$ & $33 \%$ & $52 \%$ \\
\hline Non-Seeker & $4 \%$ & $19 \%$ & $76 \%$ \\
\hline
\end{tabular}

Table 2.4.3 Percentage correctly classified - All data

Three Bins - Intervals Based on Data Inspection

Lastly, the binning method is used to simply validate earlier findings in Section 2.1 based on data inspection. Three bins were used. Specifically, cases less than 280 went into Bin 1, 280 to 301 in Bin 2, and greater than 301 in Bin 3. As expected, results are reflective of that which was reported in Section 2.1. For additional clarity, Table 2.4 .4 presents the results in a manner comparable to the previous tables in this section. 


\begin{tabular}{|c|c|c|c|}
\hline Bin & 1 & 2 & 3 \\
\hline Score & $\begin{array}{l}-157.95 \\
\text { to } \\
279.90\end{array}$ & $\begin{array}{l}280.14 \\
\text { to } \\
301\end{array}$ & $\begin{array}{l}301.10 \\
\text { to } \\
557.14\end{array}$ \\
\hline$n$ & 1009 & 102 & 451 \\
\hline & $\begin{array}{c}\% \text { of category in } \\
\text { bin }\end{array}$ & & \\
\hline Seeker & $76 \%$ & $5 \%$ & $19 \%$ \\
\hline Passive & $46 \%$ & $10 \%$ & $44 \%$ \\
\hline Non-Seeker & $19 \%$ & $5 \%$ & $75 \%$ \\
\hline
\end{tabular}

\section{Discussion}

While seekers and non-seekers are classified fairly well, there is still difficulty classifying the passives based on the loyalty score using a visual binning approach to intervals.

The motivation for identifying passives is that an employer can take measures to enhance loyalty if the passive is valuable to the organization. Perhaps supervisory issues are affecting the passive's likelihood to seek, or there are issues related to the company (e.g., pride, honesty, valuable products, competitive salary, good benefits). Alternately, if the passive has not been performing to management expectations, or has a history of inappropriate behavior, then measures should be taken to remove the passive from the organization versus further training and development.

This leads to efforts in Section 2.5 where cluster analysis techniques are used to attempt to more reliably classify our respondents as seekers, passives, or non-seekers. 


\subsection{Classification Based on Intervals - Cluster Analysis}

Reasoning and Goal

Cluster analysis is a method of exploring the data to see which groups are present. Here the loyalty score means are investigated along with the distances that respondent scores fall from the means of the subclasses of seekers, passives and non-seekers. Because there may be many underlying groups, or potential clusters, in a dataset, the researcher typically makes a priori decisions to simplify the process (Gentle 238). This might include deciding on the number of clusters as in K-means clustering, or comparing the computational challenges for the varied methods and selecting the one that works best for the application.

Agglomerative hierarchical clustering has three methods including single linkage, sometimes referred to as nearest neighbor, complete linkage, and average linkage. In $K$-means clustering cases are assigned to a cluster with the nearest mean. One may also consider Ward's method in which the distance measure is based on change in explained variance, $R^{2}$. In general, look for consistency in clustering methods.

In hierarchical clustering the interpoint and intercluster distance is defined. The interpoint distance is the square root of the sum of the distances squared, or Euclidean distance

$$
d_{E}\left(x_{1}, x_{2}\right)=\sqrt{\sum_{j=1}^{p}\left(x_{1 j}-x_{2 j}\right)^{2}}
$$

where $\left.x_{1}=x_{11}, \ldots x_{1 p}\right)^{\prime}$ and $\left.x_{2}=x_{21}, \ldots x_{2 p}\right)^{\prime}$

are $p$-dimensional vectors in $R^{p}$. The intercluster distance is a function of the interpoint distances between pairs of points, and each point comes from a different cluster. In single linkage, this is the distance between the two closest points, for complete linkage the farthest two points, and in average linkage the average distance between every pair of points.

The basic clustering algorithm follows:

1. Start with $N$ clusters, each containing a single entity and $N x N$ symmetric matrix of distances (or similarities)

$$
\left.D=d_{i k}\right\}
$$

2. Search the distance matrix for the nearest (most similar) pairs of clusters. Let the distance between most similar clusters $U$ and $V$ be $d_{U V}$. 
3. Merge clusters $U$ and $V$. Label new cluster $(U V)$. Update entries in the distance matrix.

4. Repeat steps 2 and 3 a total of $N-1$ times. Record the identity of clusters that are merged and the levels of distances, or similarities, at which the merge takes place.

In single linkage, groups are fused according to the distance between their nearest members. For the single linkage algorithm inputs can be distances or similarities between pairs. Groups are formed from individual entities by merging the "nearest neighbors," meaning the smallest distance or largest similarity. To find the smallest distance $\left.D=d_{i k}\right\}$, merge corresponding objects $U$ and $V$ to generate cluster $(U V)$. Distances between $(U V)$ and any other cluster $W$ are

$$
d_{(U V) W}=\operatorname{nin}\left\{d_{U W} d_{V W}\right\}
$$

where $d_{(U W)}$ and $d_{(V W)}$ are distances between nearest neighbors of clusters $U$ and $W$ and clusters $V$ and $W$, respectively.

Complete linkage is similar to single linkage except that the distance between clusters is determined by the distance between two elements, one from each cluster, that are most distant. All items in the cluster are within some maximum distance with minimum similarity. In the algorithm for complete linkage find the minimum entry in $\left.D=d_{i k}\right\}$, and merge the corresponding items to generate cluster $(U V)$. The distances between $(U V)$ and any other cluster $W$ are

$$
d_{(U V) W}=\operatorname{nax}\left\{d_{U W}, d_{V W}\right\}
$$

where $d_{U W}, d_{V W}$ are the distances between the most distant of clusters $U$ and $W$ and cluster $V$ and $W$, respectively.

For average linkage, the distance between two clusters is the average distance between all pairs. One member of each pair belongs to each cluster. The input to the algorithm may be distances or similarities to group objects or variables. Search the distance matrix $D=d_{i k}$ \} to find the most similar (nearest) objects $U$ and $V$. Them, merge them to form cluster $(U V)$. The distances are

$$
d_{(U V) W}=\frac{\sum_{i} \sum_{k}}{N_{(U V)} N_{W}}
$$


where $d_{i k}$ is the distance between object $i$ in cluster $(U V)$ and object $k$ in the cluster $W$, and $N_{(U V)}$ and $N_{(W)}$ are the number of items in clusters (UV) and $W$.

The Ward method is distinct from all the other methods discussed thus far. It uses and analysis of variance approach to evaluate the distances between clusters. It attempts to minimize the sum of squares of any two (hypothetical) clusters formed at each step. It is regarded as very efficient, however, as it tends to create clusters of small size. This method will be applied in the second essay analyzing on college student leadership behaviors.

With the $K$-means method, each item is assigned to a cluster having the nearest centroid (mean). The algorithm step 1) partitions items into $K$ initial clusters. It then in step 2) proceeds through the items, assigning them to clusters with the nearest mean. The Euclidean distance is used with standardized or unstandardized observations. The mean is recalculated for the cluster receiving the new observation as well as for the cluster losing the observation. Step 2 is repeated until no more reassignments take place.

The objective of $K$-means clustering is to find and segment observations into a preset number of groups, $k$, which will minimize the variation within each group, and each group may have different variation. The variation of the $j^{\text {th }}$ variable in the $g^{\text {th }}$ group is measured by the within-sum of-squares

$$
s_{j(g)}^{2}=\frac{\left.\sum_{i=}^{n_{g}} x_{i j(g)}-\bar{x}_{j(g)}\right)^{2}}{n_{g}-}
$$

where $n_{g}$ is the number of observations in the $g^{\text {th }}$ group, and $\bar{x}_{j(g)}$ is the mean of the $j^{\text {th }}$ variable in the $g^{\text {th }}$ group. There are $m$ of these quantities. The variation of observations in the $g^{\text {th }}$ group is chosen as a linear combination of the sums-of-squares for all $m$ variables. Partitions are created such that the pairwise dissimilarities between those assigned to the same cluster tend to be smaller than those indifferent clusters (Hastie, Tibshirani, Friedman, 459).

Hastie, Tibshirani and Friedman (p. 461) suggest that the $K$-means algorithm is one of the most popular iterative descent clustering methods, intended for use with quantitative variables. The squared Euclidean distance is chosen as the dissimilarity measure (Hastie et al., 461).

$$
d\left(x_{i}, x_{i^{\prime}}\right)=\sum_{j=1}^{p}\left(x_{i j}-x_{i^{\prime} j}\right)^{2}=x_{i}-x_{i^{\prime}} \|^{2}
$$


Application to the Loyalty Score

The first cluster analysis deals with only one variable, the loyalty score, which is segmented into a preselected number of clusters, a manageable nine. The $K$-means clustering method was used to generate the clusters. Table 2.5.1 reports the exact interval of the assigned cluster, the $n$ respondents who fall into the cluster, the $n$ in each seeking category within the cluster, and the percentage of the total category identified.

\begin{tabular}{|c|c|c|c|c|c|c|c|c|c|}
\hline Cluster & 1 & 2 & 3 & 4 & 5 & 6 & 7 & 8 & 9 \\
\hline Score & $\begin{array}{l}-157.95 \\
\text { to } \\
-55.53\end{array}$ & $\begin{array}{l}-48.83 \\
\text { to } \\
27.63\end{array}$ & $\begin{array}{l}29.12 \\
\text { to } \\
113.71\end{array}$ & $\begin{array}{l}114.41 \\
\text { to } \\
199.56\end{array}$ & $\begin{array}{l}199.93 \\
\text { to } \\
280.47\end{array}$ & $\begin{array}{l}281.32 \\
\text { to } \\
349.22\end{array}$ & $\begin{array}{l}349.99 \\
\text { to } \\
407.75\end{array}$ & $\begin{array}{l}409.15 \\
\text { to } \\
470.75\end{array}$ & $\begin{array}{l}493.06 \\
\text { to } \\
577.14\end{array}$ \\
\hline \multirow[t]{2}{*}{$n$} & 35 & 86 & 198 & 303 & 391 & 319 & 180 & 47 & 3 \\
\hline & $\begin{array}{c}\text { \% of } \\
\text { category } \\
\text { in } \\
\text { cluster }\end{array}$ & & & & & & & & \\
\hline Seeker & $3 \%$ & $8 \%$ & $16 \%$ & $23 \%$ & $26 \%$ & $16 \%$ & $7 \%$ & $1 \%$ & \\
\hline Passive & $.02 \%$ & $.07 \%$ & $6 \%$ & $15 \%$ & $24 \%$ & $31 \%$ & $17 \%$ & $6 \%$ & $.02 \%$ \\
\hline $\begin{array}{l}\text { Non- } \\
\text { Seeker }\end{array}$ & & $1 \%$ & & $3 \%$ & $15 \%$ & $25 \%$ & $2 \%$ & $13 \%$ & $2 \%$ \\
\hline
\end{tabular}

Table 2.5.1 K-means clustering of loyalty scores

The results for the $K$-means cluster analysis on the loyalty scores are particularly more helpful in identifying seeking status. However, as discussed earlier, $76 \%$ of the seekers have scores less than or equal to approximately 280 , and the $K$-means method enables one to inspect the classifications more carefully based on loyalty score.

Using just the factors in the cluster analysis, pre-selecting only three clusters yields poor results. Two of the clusters (cluster 2 and cluster 3 in Table 2.5.2) have loyalty scores that overlap too much to consider the score helpful in discriminating classes (Table 2.5.2). 


\begin{tabular}{|c|c|c|c|}
\hline Cluster & 1 & 2 & 3 \\
\hline Loyalty Score & $\begin{array}{l}-159.02 \\
\text { to } \\
209.41\end{array}$ & $\begin{array}{l}124.96 \\
\text { to } \\
557.37\end{array}$ & $\begin{array}{l}125.50 \\
\text { to } \\
452.79\end{array}$ \\
\hline \multirow[t]{2}{*}{$n$} & 518 & 406 & 638 \\
\hline & $\%$ of category in cluster & & \\
\hline Seeker & $42 \%$ & $22 \%$ & $36 \%$ \\
\hline Passive & $17 \%$ & $34 \%$ & $49 \%$ \\
\hline Non-Seeker & $4 \%$ & $41 \%$ & $55 \%$ \\
\hline
\end{tabular}

Table 2.5.2 K means clustering based on factors

Investigating a three-cluster solution using the loyalty scores yields an interesting result regarding non-seekers. It is known (see Section 2.1) that $75 \%$ of the non-seekers have scores greater than or equal to 301. Cluster analysis reveals $85 \%$ greater than 269 .

Cluster analysis suggests a passive is not likely to have a score less than 117 (Table 2.5 .3 ). Nearly $60 \%$ of the passives had loyalty scores over 269 . Recall that if the score is greater than 301, it was suggested in Section 2.1 that they were most likely to be non-seekers. Further, the mean loyalty score for passives is 273 .

\begin{tabular}{|c|c|c|c|}
\hline Cluster & 1 & 2 & 3 \\
\hline Loyalty Score & $\begin{array}{l}-159.02 \\
\text { to } \\
116.74\end{array}$ & $\begin{array}{l}117.89 \\
\text { to } \\
269.89\end{array}$ & $\begin{array}{l}269.10 \\
\text { to } \\
557.37\end{array}$ \\
\hline \multirow[t]{2}{*}{$n$} & 326 & 627 & 609 \\
\hline & $\%$ of category in cluster & & \\
\hline Seeker & $28 \%$ & $44 \%$ & $28 \%$ \\
\hline Passive & $7 \%$ & $35 \%$ & $57 \%$ \\
\hline Non-Seeker & $1 \%$ & $14 \%$ & $85 \%$ \\
\hline
\end{tabular}

Table 2.5.3 K means clustering based on loyalty scores, pre-designated 3 clusters

This suggests that scores between 269 and 301 are likely to indicate passive status, and careful interpersonal analysis is required by the manager for employees scoring in this range. Given that $85 \%$ of the non-seekers are found with scores above 269 , one might infer that the passive with a score over 269 (yet under 301) can be effectively transitioned to non-seeking status by the manager who focuses on improved supervisory experiences for the passive, as well as the elements comprising the company factor. 


\section{Discussion}

Cluster analysis did not prove to be a particularly fruitful technique for classification based on intervals of the loyalty score. However, $85 \%$ of the non-seekers were assigned to the same cluster based on loyalty scores over 269 , versus the threshold of 280 recommended in Section 2.1 (where $75 \%$ of non-seekers had scores over 280). Also, a bit more information regarding the loyalty scores of passives was interpreted. It was suggested that passives are not likely to have scores lower than 117, and those falling in the 269 to 301 range should be counseled by management in an effort to secure their loyalty to the organization, and transition them to non-seeking status.

The upcoming Section 2.6 will discuss a more effective method for discerning seeking categories, discriminant analysis. 


\subsection{Classification Using Discriminant Analysis}

Next, a more practical and intuitive approach is taken to determining what elements of the job experience might serve as strong catalysts for a change in seeking status, ultimately leading to better classification of an employee as a seeker, passive, or non-seeker. Earlier, the many significant differences between subclasses were discussed. Particularly noted was the substantiality of the differences between all groups on perceptions of pride in the company for which they worked and its honesty. It was suggested that the supervisor's support of work/life balance was a potential catalyst to transition from nonseeker to passive. These are just a few of the intuitions that can be validated with the classification and analysis techniques within the discipline of discriminant analysis which helps the researcher build a predictive model for group membership.

Overview of Discriminant Analysis

The model can be designed to differentiate between two groups, and classify members accordingly, or for more than two groups in which case the process is called multiple discriminant analysis. The model in the case of more than two groups is composed of a set of discriminant functions that are based on linear combinations of independent predictor variables that provide the best discrimination between the groups. Functions are generated from a sample of cases for which group membership is known, and then validated by applying them to new cases that have measurements for the predictor variables but have unknown group membership.

The job-seeker data lends itself to discriminant analysis as the aim is to classify employees as members of one of three groups (seekers, passives or non-seekers). Discriminant analysis is an appropriate statistical tool when the dependent variable is categorical and independents are numeric (Hair, Black, Babin, and Anderson, p. 239).

The process involves deriving a variate, the discriminant function, which is a linear combination of two or more independent variables that best differentiate, or discriminate, between the cases (e.g., employees) in each of the job-seeker status categories which are predefined, often referred to as defined $a$ priori. Weights are calculated for each independent variable with the intent of maximizing the differences between groups. Essentially, this means weighting by the between-group variance relative to the within-group variance. 
The form follows:

$$
Z_{j k}=\imath+V_{1} X_{1 k}+V_{2} X_{2 k}+\ldots+V_{n} X_{n k}
$$

where

$$
\begin{aligned}
& Z_{j k}=\text { discriminant } Z \text { score of discriminant function } j \text { for object } k \\
& a=\text { intercept } \\
& W_{i}=\text { discriminant weight for independent variable } i \\
& X_{i k}=\text { independent variable } i \text { for object } k
\end{aligned}
$$

Unique to discriminant analysis versus other multivariate techniques, is that each object, or person, may have more than one discriminant score based on multiple functions depending on the number of categories being discriminated. The number of functions is determined as number of groups (or categories) minus 1 .

Hair et al clarify that discriminant analysis is used to test the hypothesis of equality of group means for a set of independent variables for two or more groups. The group mean, or centroid, is the average of discriminant scores for all cases (persons, employees, objects, etc.) within a specific group. One centroid is determined for each category, or group, and this centroid is an indication of the most typical location of any member from a particular group.

Statistical significance of the discriminant function is assessed by comparing the distance between group centroids. If the distributions of the discriminant scores for the groups overlap substantially, then the function does not discriminate well. Alternately, if the distributions overlap minimally, then the function effectively discriminates between groups.

Consider the following geometric represntation of a two-group discrimant function (Figure 2.6.1). Ellipses A and B represent two groups, and variables $X_{1}$ and $X_{2}$ are measurements for each member of each group. Envision the scatterplot representing the association of $X_{1}$ and $X_{2}$ for each member of groups A and B. In Figure 2.6.1 stars represent variable measurements for members of group A and dots represent variable measurements for gorup B. The ellipses enclose for example $95 \%$ of the points in each group. Draw a line through the point of intersection where there are both stars and dots, and project that line to a new axis, $\mathrm{Z}$. The overlap in univariate distributions $\mathrm{A}^{\prime}$ and $\mathrm{B}^{\prime}$, where the arrow points, is less than would be obtained by any other line drawn through the ellipses formed by the scatterplots (Green, Tull, Albaum, 1988). 


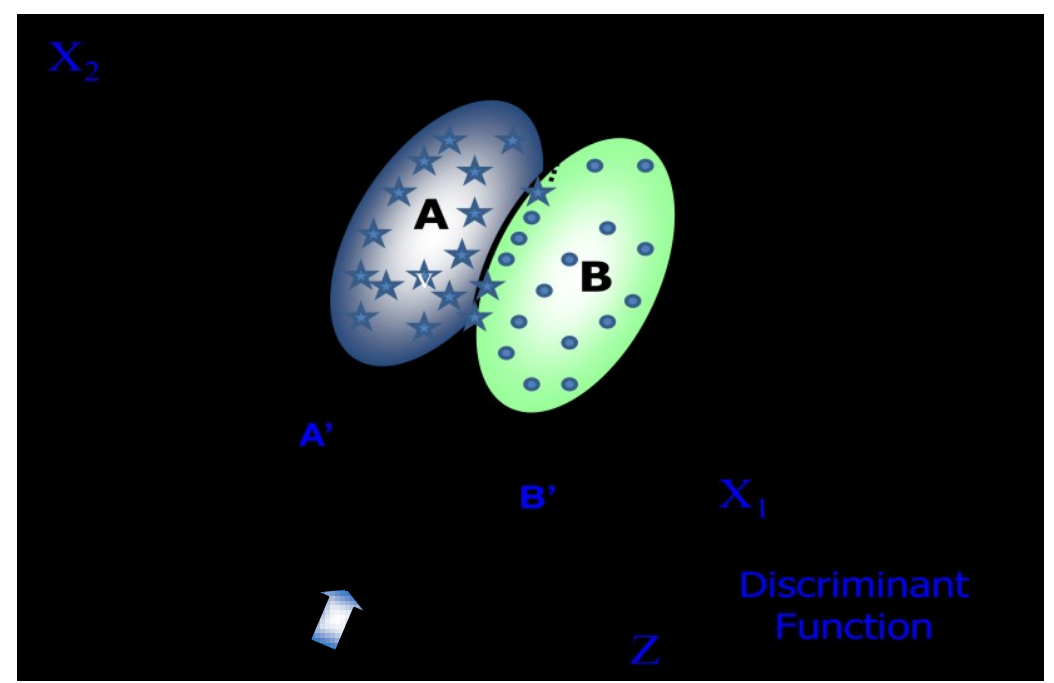

Figure 2.6.1 Two-group discriminant function

Note that on the $\mathrm{Z}$ axis are the discriminant scores, expressed as a single number, which represent the linear combination of original variables $X_{1}$ and $\mathrm{X}_{2}$, condensing the profile information from both groups, inclusive of the difference information.

After deriving the linear combinations of the independent variables, there are discriminant scores for each object in each group. The aim is to maximize the variance between groups and minimize variance within groups to separate them most efficiently (Hair, et al, p. 245).

Applying and interpreting discriminant analysis is similar to regression in that information is extracted from independent variables to predict a dependent variable. The difference is that in discriminant analysis the dependent variable is categorical, versus metric as in regression. Also, consider conceptual similarities with multivariate analysis of variance (MANOVA) where the dependent variable is metric and independents are categorical. In discriminant analysis, the inverse is true. Metric independent variables are used to predict or classify cases into the categories of the dependent variable.

In regression, the researcher typically reviews correlations to select viable candidate independent variables, whereas in discriminant analysis, one considers mean differences between the groups as a directional starting point. Identifying significant differences between groups is intrinsic to beginning to develop the discriminant function. 


\section{Linear Discriminant Analysis}

Hastie, Tibshirani and Friedman (2001) suggest that decision theory for optimal classification requires class posteriors $\operatorname{Pr}(G \mid X)$. Consider $f_{k}(x)$ the class-conditional density of $X$ in class $G=k$, and the probability of $k$ as the prior probability of class $k$ with $\sum_{k=1}^{K} \pi=1$. Applying Bayes theorem gives

$$
\operatorname{Pr}(G=k \mid X=x)=\frac{f_{k}(x) \pi}{\sum_{l=1}^{K} f_{l}(x) \pi}
$$

Thus, if the $\pi_{l}$ are known, having $f_{k}(x)$ is virtually equivalent to having the quantity $\operatorname{Pr}(G=\imath \mid X=\imath)$.

Model each class density as multivariate Gaussian:

$$
f_{k}(x)=\frac{1}{\left(2 \pi^{p / 2} \mid \sum_{k}^{1 / 2}\right.} e^{-1 / 2(x-\mu)^{T} \sum_{k}^{-1}(x-\mu)}
$$

Linear discriminant analysis (LDA) is used when equal covariance matrices are assumed for the classes. Test homogeneity of covariance matrices with Box's M test which will be discussed later. The comparison of two classes, say $k$ and $l$, yields an equation linear in $x$, which can be seen by looking at the log-ratio:

$$
\begin{gathered}
\log \frac{\operatorname{Pr}(G=\imath \mid X=\imath)}{\operatorname{Pr}(G=\mid X=\imath)}=\lg \frac{f_{k}(x)}{f_{l}(x)}+\lg \frac{\pi}{\pi} \\
= \\
\log \frac{\pi}{\pi}-\frac{1}{2}(\mu+\imath)^{T} \sum^{-}(\mu-\ell)+i^{T} \sum-(\mu-\imath) .
\end{gathered}
$$

Equal covariance matrices allow for cancellation of the normalization factors, as well as the quadratic part in the exponents. Thus the linear log-odds function suggests that the decision boundary between the classes $k$ and $l$ (the set where $\operatorname{Pr}(G=\imath \mid X=\imath)=\operatorname{l} \mathrm{r}(G=\mid X=\imath))$, is linear in $x$; in $p$ dimensions a hyperplane (Hastie, et al, p 86). 
Figure 2.6.2 attempts to show three Gaussian distributions, with the same covariance and different means. The 95\% confidence contours and class centroids are included. There are 3 classes and 2 discriminating functions, $p$ $=2$. The Bayes decision boundaries between each pair of classes are represented by the dashed lines, while the fitted LDA decision boundaries separating all three classes are the solid lines (a subset of the pair boundaries). These decision boundaries are not the perpendicular bisectors of the line segments joining the centroids, which would be the case if the covariance $\boldsymbol{\Sigma}$ were spherical $\sigma^{2} \mathbf{I}$, and the class priors were equal (Hastie, et al, p. 87).

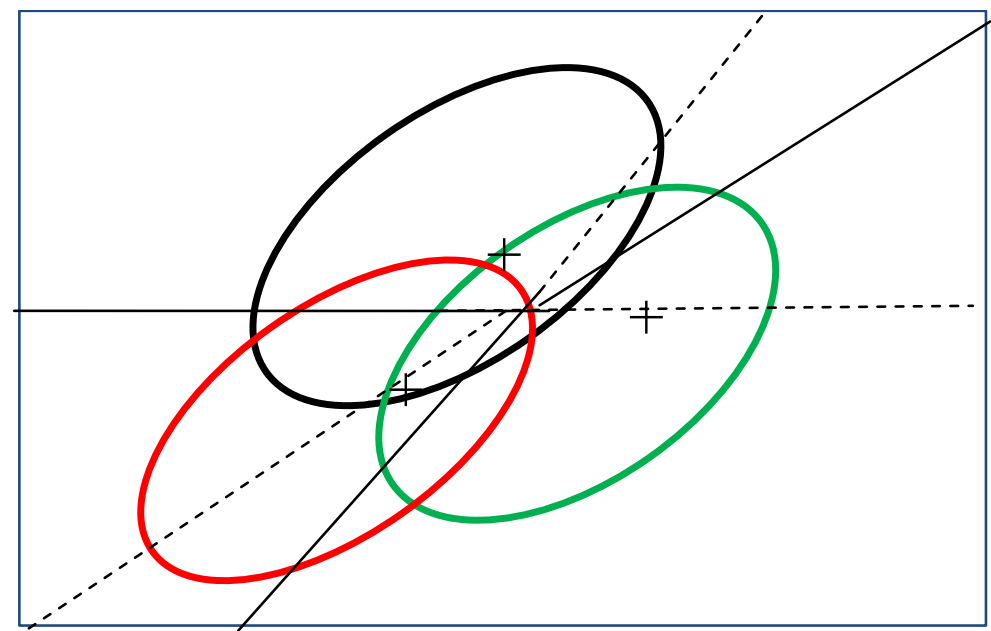

Figure 2.6.2 Solid lines representing fitted LDA boundaries, 3 Gaussians

From the log ratio of the classes as defined earlier, one can then define the linear discriminant functions as

$$
\delta(x)=x^{T} \sum^{-1} \mu-\frac{1}{2} \mu \sum^{-1} \mu+\log \pi
$$

This is equivalent to the description of the decision rule, with $G(x)=\operatorname{rg} \max _{k} \delta(x)$. Since the parameters of the Gaussian distributions are likely to be unknown in practice, they are estimated using training data where

$$
\begin{aligned}
& \pi=\mathrm{V}_{k} / N, \text { where } N_{k} \text { is the number of class } k \text { observations } \\
& \mu=\sum_{g_{i}=k} x_{i} / N_{k} \\
& \hat{\sum} \sum_{=} \sum_{=}\left(x_{i}-\mu\right)\left(x_{i}-\mu\right)^{T} /(N-\zeta) .
\end{aligned}
$$

If the covariance matrices are unequal, which can be determined with Box's M test, there are not the convenient cancellations, and the results of our analysis will utilize quadratic discriminant functions (QDA), 


$$
\delta(x)=-\frac{1}{2}-\log \mid \sum_{k}-\frac{1}{2}(x-\mu)^{T} \sum_{k}^{-1}(x-\mu)+\log \pi
$$

Thus, the decision boundary between each $k$ and $l$ pair is described by a quadratic equation $\left.\left\{x: \delta(x)=\mathcal{S}^{\prime} x\right)\right\}$.

\section{Box's M Test}

A key question then, in determining the specific procedure to be implemented, is whether the classes have a common covariance matrix $\sum_{k} \forall k$. As mentioned earlier, homogeneity of class covariance matrices is tested with Box's M test. Box (1949) derived a test statistic based on the likelihood-ratio test, for moderate to small sample sizes, with an $F$ approximation used to compute its significance.

The $j^{\text {th }}$ set of $r$ dependent variables in the $i^{\text {th }}$ cell are

$$
y_{.,}^{\prime}=c_{, j}^{\prime} B+,
$$

where

$$
\begin{gathered}
e_{i j} \sim N_{r}\left(0, w_{i j}^{-1} \sum_{i}\right) \\
\text { for } i=, \ldots, g \text { and } j=, \ldots, n_{i}
\end{gathered}
$$

and where $g$ is the number of cells with non-singular covariance matrices, and $w_{i j}$ is the regression weight associated with $y_{i j}$, and $w_{i j}>0$.

To clarify, one tests the equality of the covariance matrices of the independent variables, across the groups, or classes, of the dependent variable (Hair et al, p. 236). If the test does not exceed the critical value, it is deemed insignificant and equality of covariance matrices is assumed. If the test indicates a statistically significant difference between independent variable covariance matrices across groups, then the discriminant analysis proceeds, using techniques for separate covariance matrices.

Box's $\mathrm{M}$ is said to be particularly sensitive to deviations from normality (Statsoft Electronic Textbook, 2008), the size of the covariance matrices, and number of groups (Hair et al, 254). A conservative approach is suggested (0.01 instead of 0.05) when determining if true differences are present within the groups. As sample size increases, or the number of groups, or the number of independent variables increases, even more conservative levels are considered acceptable. 
Classification can be negatively impacted by unequal covariance matrices. Hair et al suggest that if sample sizes are small and covariance matrices unequal, the statistical significance of the estimation process is compromised. What is common, is unequal covariances among groups of reasonable sample size, yet where observations are over classified into groups with larger covariance matrices. Either the sample size can be increased, or separate, group-specific, covariance matrices can be used for classification. In this case, cross-validation is required. Briefly, cross-validation assures internal validity, estimating the discriminant model by leaving out one case then predicting that case with the estimated model. Continuing the process for each observation, no one observation ever influences the model that predicts its classification.

\section{Assumptions}

In addition to a preference for multivariate normality of the independent variable, and homogeneity of variance and covariance structures of dependent variable groups as discussed, the researcher must be wary of multicollinearity as measured by tolerance values. Essentially, independent variables in the model should not be predictable by one another, thus adding limited insight into the explanation of the dependent variable, or class in the case of discriminant analysis. Review tolerance values to guard against an illconditioned matrix which is one with redundant variables being used to discriminate between categories. Tolerance is measured as $1-R^{2}$ of a specific variable with all other variables included in the current model, indicating the proportion of variance that is unique to the specific variable. The measure should approach 1, and variables with measurements approaching 0 considered redundant. Violations of multivariate normality can impact estimation, while unequal covariance matrices yield their effects on classification, and multicollinearity among independent variables adversely affects interpretation.

\section{Application to Job-Seeker Data}

Applying the concepts discussed thus far to the job-seeker dataset, the factor scores and their interaction effects are considered as independent variables to potentially predict job-seeking status. Starting with the four factor scores for each case, namely SUPERVISOR, COMPANY, OPPORTUNITY and QUALITY of WORK-LIFE, and in order to understand the impact of interaction among these variables, six new variables were created to represent such interaction: 


\section{SUPV*COMP \\ SUPV*OPPTY \\ SUPV*QWL \\ COMP*OPPTY \\ COMP*QWL \\ OPPTY*QWL}

Normality Issues

Shapiro-Wilk's test for normality suggests the factor scores and the interaction variables not normally distributed. In all cases, $p$ values were essentially 0 , thus we reject the null of normality.

\begin{tabular}{|lcc|}
\multicolumn{1}{c}{ Factor } & W-statistic & p-value \\
\hline SUPERVISOR & .9859 & $p=0$ \\
\hline COMPANY & .9770 & $p=0$ \\
\hline OPPORTUNITY & .8752 & $p=0$ \\
\hline QUALITY OF & .9742 & $p=0$ \\
WORK-LIFE & & \\
\hline SUPV*COMP & .8886 & $p=0$ \\
\hline SUPV*OPPTY & .8146 & $p=0$ \\
\hline SUPV*QWL & .9124 & $p=0$ \\
\hline COMP*OPPTY & .7828 & $p=0$ \\
\hline COMP*QWL & .9213 & $p=0$ \\
\hline QWL*OPPTY & .5843 & $p=0$ \\
\hline
\end{tabular}

Table 2.6.1 Shapiro Wilk's test does not suggest normality.

Garson (2009) suggests that discriminant analysis will be robust against violations of normality if the smallest group has more than twenty cases and the number of independents is fewer than six. Each of the seeking categories far exceeds the data quantity requirement, yet the final discriminant model uses seven independents, two more than the recommended number. We move forward with an understanding that the departures from normality may negatively impact estimation.

Variable Selection and Procedure

The four constructs (factors) plus their interaction effects (as six new variables) were used as independent variables in the discriminant analysis procedure. The grouping, or dependent, variable was job-seeking status, specifically job-seeker, passive or non-seeker. Within the discriminant analysis process, the stepwise estimation procedure was specified which estimates the discriminant function by entering the independent variables sequentially, according to the discriminatory power they add to the prediction of group membership. 
Keeping in mind the analytical process described above, first consider the significant differences between the groups (seeker, passive, non-seeker) given the independent variables used in building the discriminant function for classification. Table 2.6.2 provides the significant differences on the 10 independent variables to be used in the analysis -4 factors plus 6 interaction variates, with regard to seekers versus passives. For simplicity, only the variates with significant differences between the means of two groups are reported, along with their respective t-statistics derived from the independent samples t-test, and the mean difference.

\begin{tabular}{|llllll|} 
& $\begin{array}{l}\text { Seekers } \\
\text { Group 1 } \\
\mathbf{n = 1 0 5 1}\end{array}$ & $\begin{array}{l}\text { Passives } \\
\text { Group 2 } \\
\mathbf{n = 4 1 0}\end{array}$ & T Stat & $\begin{array}{l}\text { Sig. 2 } \\
\text { tailed }\end{array}$ & Mean diff \\
\hline COMPANY & -.236 & .413 & -13.019 & .000 & -.650 \\
\hline SUPERVISOR & -.140 & .228 & -6.811 & .000 & -.369 \\
\hline $\begin{array}{l}\text { QUALITY of } \\
\text { WORK-LIFE }\end{array}$ & .081 & -.147 & 3.980 & .000 & .228 \\
\hline
\end{tabular}

Table 2.6.2 Significant differences seekers vs. passives on 4 factors, 6 interactions

The table suggests substantial differences between seekers and passives on perceptions of COMPANY, followed by SUPERVISOR, and QUALITY of WORK-LIFE. The implication is that these mean differences will yield good discriminators between these two groups.

Next, consider significant differences between passives and non-seekers with regard to the 10 variates that will be used in the discriminant analysis (Table 2.6.3). Recall that seekers and passives evidenced significant differences on only three factors (COMPANY, SUPERVISOR, and QUALITY of WORKLIFE), whereas groups 2 and 3, passives and non-seekers, evidence more differences based on interaction effects. From this, one might infer that the passive candidate transitions to seeking if a significant change in the company, supervisor or quality of work-life is perceived.

Further, a non-seeker may move to a passive (would consider changing jobs given the right situation), and vice-versa, if a significant change is perceived in both quality of work-life and alternate opportunities, simultaneously. The same holds true in that there is a significant difference in perceptions of nonseeker vs. passive regarding the interaction effects of COMPANY and OPPORTUNITY. It is suggested that these factors and their interactions can serve as a catalyst for transition between seeking states. The discriminant analysis enables one to ascertain the variates that most effectively classify a case (i.e. employee). 


\begin{tabular}{|llllll|} 
& $\begin{array}{l}\text { Passives } \\
\text { Group 2 } \\
\mathbf{n = 4 1 0}\end{array}$ & $\begin{array}{l}\text { Non- } \\
\text { seekers } \\
\text { Group 3 } \\
\mathbf{n = 9 3}\end{array}$ & T Stat & $\begin{array}{l}\text { Sig. 2 } \\
\text { tailed }\end{array}$ & Mean diff \\
\hline QWL*OPPTY & -.044 & .749 & -2.68 & .008 & -.787 \\
\hline COMP*OPPTY & .078 & -.435 & 2.84 & .005 & .514 \\
\hline OPPORTUNITY & -.033 & -.490 & 2.95 & .004 & .456 \\
\hline COMPANY & .413 & .851 & -5.56 & .000 & -.438 \\
\hline SUPV*COMPANY & .052 & .453 & -3.81 & .000 & -.400 \\
\hline SUPERVISOR & .228 & .578 & -4.23 & .000 & -.350 \\
\hline
\end{tabular}

Table 2.6.3 Significant differences passives vs. non-seekers on 4 factors, 6 interactions

Returning to the three-group discriminant analysis, recall that given three groups, the result will be two discriminant functions which are dimensions, each separate and distinct from the other, that separate the groups and will ultimately be used to classify new cases into group membership.

Hair et al, suggest overall sample size should be a ratio of 20 observations for each predictor variable. The approach taken here is quite conservative given that if all 10 variables are entered and used in the stepwise analysis, 200 cases would be needed. This dataset is comprised of a total of 1554 (1051 group 1 seekers, 410 group 2 passives, and 93 group 3 non-seekers). Additionally the practical guideline of at least 20 observations per category is maintained. This is particularly true with regard to cross-validation and hold-out (also called validation) samples. Subsamples are created for purposes of estimation, and both internal and external validation. The first subsample is the analysis or estimation sample, typically comprised of $70-75 \%$ of the dataset, with a second set of $25-30 \%$ held out at random. This second set, the validation or hold-out sample, is used to apply the discriminant function(s), and subsequently classify a group of new cases without having used those cases to estimate the functions, thus reducing bias and assessing external validity. As discussed earlier, internal validity is achieved with a leave-one-out crossvalidation process, in which each case is individually predicted by the others yielding a more conservative estimate of classification accuracy (often by just a few percentage points, but the results add insight by category).

An additional consideration at this point is a priori knowledge of group membership, and the size of groups. The job-seeker dataset adds a level of complexity because the group sizes in the sample are substantially varied, and not necessarily representative of the population. Further, respondents completed the survey within the context of an online job-search engine, so the total sample is more representative of job-seekers. However, because the overall research objective is to be able to identify seeking status without 
directly asking the question, and there are 3 groups into which the respondent will naturally fall (seeker, passive, non-seeker), utilize equal priors for all groups (i.e., .33 for each group). Hair et al (p. 261) support a conservative approach of equal priors when the researcher is unsure whether the observed proportions in the sample are representative of the population proportions.

In an optimal situation where this analysis is applied in a specific organizational setting, or is industry-specific, or applied to a particular geographic region or country, the researcher would want to ensure greater randomness in the sampling procedure, and apply any a priori knowledge of current levels of job-seekers, passives and non-seekers. Typically, these proportions are somewhat dynamic. At the time of data collection in early 2007 , prior to a serious weakening in the global economic condition, jobseeking or passiveness while in a current position was likely to be more prevalent, whereas secure positions (yielding more non-seekers) are likely to be more appreciated in an economic downturn. It is important to note the value of the less biased dataset used here in that these findings are likely to be more representative of the common condition.

Another issue is that Box's $\mathrm{M}$ is, in fact, significant given the job-seeker dataset, suggesting the use of separate covariance matrices, which in turn negatively impacts classification accuracy. As mentioned earlier, Box's M is sensitive to large sample sizes, large numbers of predictors, and large numbers of groups, all of which are basically true here. Hair et al suggest a less conservative approach to maintaining the assumption of equal covariance matrices, and Statsoft researchers go so far as to suggest that because Box's M is particularly sensitive to deviations from multivariate normality (of which there is evidence), it should not be taken "too seriously."

The classification results for both methods, pooled covariance matrices and separate, were validated by running the discriminant analysis three times, producing an average result discussed in the upcoming Classification and Assessment of Accuracy section. Overall the use of separate covariance matrices resulted in negligible impact on classification of seekers, with a $1.3 \%$ reduction in accuracy; a more serious negative effect on classifying passives, at a $3.3 \%$ reduction in accuracy; and roughly a $2 \%(1.96)$ decrease in ability to classify non-seekers. These results are based on the most conservative holdout validation sample which uses the estimation (or analysis) set to derive the discriminant function(s), and subsequently classify the hold-out sample cases without having used those cases to estimate the functions. Both methods, for all groups, surpass the standard classification hit ratio criterion of $41.25 \%$ 
(Hair et al, p. 265) for one-fourth better than chance accuracy for discriminating between three groups (see Table 2.6.11 and Table 2.6.12)

Two computational methods are offered in developing the discriminant functions. Either all independent variables can be entered simultaneously, where discriminatory power is tested with Wilks' lambda, or stepwise where Mahalanobis' $D^{2}$ measures most appropriately assess fit. In most cases both methods are tested, but since ten variables are under consideration regarding the job-seeker data, the stepwise method is employed. Given utilization of the stepwise method, the advantage of using the Mahalanobis' $D^{2}$, which is based on generalized squared Euclidean distance that adjusts for unequal variances, is that it is computed in the original space of the predictor variables rather than a collapsed version as in other measures (Hair, et al, p. 258). The measure is based on correlations between variables and is useful in identifying similarities of an unknown sample set to a known one. It is different from the Euclidean distance because of the consideration of correlations and that it is scale invariant.

More specifically, Mahalanobis distance, also called quadratic distance, measures the separation of groups of objects using group means, and covariance matrix $S$ (Mahalanobis 1936). The Mahalanobis distance of a multivariate vector $\left.x=x_{1}, x_{2}, x_{3}, \ldots, x_{n}\right)^{T}$, from a group of values with mean $\mu=\mu, \mu, \mu, \ldots, \mu)^{T}$ is defined as:

$$
D_{M}(x)=\sqrt{\left(x-\iota^{T} S^{-}(x-\imath\right.} .
$$

The measure becomes more important as the number of predictors increases because it does not result in reduced dimensionality, which would in turn, cause loss of information due to decreased variability in the independent variables. Thus Mahalanobis' $D^{2}$ is the preferred method when using stepwise procedures because the researcher utilizes the maximum amount of available information. Using this distance measure in the stepwise procedure allows the sequential selection of the variables with statistically significant differences across groups while maximizing the distance between the two closest groups. Thus, these variables maximize the discrimination between the most similar groups at each stage of the stepwise analysis.

\section{Model Estimation and Assessment of Model Fit}

The stepwise discriminant analysis procedure applied to the job-seeker data, yields two discriminant functions (number of groups minus one). The first function explains the largest amount of variance in the discriminant groups, 
and the second which is orthogonal and independent of the first, explains the largest amount of the remaining variance. The general task at hand then is to calculate the discriminant scores for each observation, evaluate group differences on the discriminant scores to ensure that the function does in fact discriminate effectively between groups, and then assess how well the function classifies.

The significance of the discriminant functions is validated by Wilks' lambda which provides a measure of how well each function separates cases into groups. It is equal to the proportion of total variance in the discriminant scores not explained by differences among groups. Smaller values of Wilks' lambda suggest greater discriminatory ability of the function. (Table 2.6.4).

\begin{tabular}{|l|l|l|l|l|}
\hline Wilks' Lambda & \multicolumn{5}{|l|}{} \\
\hline $\begin{array}{l}\text { Test of } \\
\text { Function(s) }\end{array}$ & $\begin{array}{l}\text { Wilks' } \\
\text { Lambda }\end{array}$ & Chi-square & df & Sig. \\
\hline 1 through 2 & .760 & 302.92 & 14 & .000 \\
\hline 2 & .984 & 17.88 & 6 & .007 \\
\hline
\end{tabular}

Table 2.6.4 Validation of significance of discriminant functions

Further, as Table 2.6.4 indicates, Chi-square values testing the null hypothesis of equality of means of the functions listed, suggest significant differences implying that the functions do far better than chance at separating the groups. Standardized discriminant function coefficients with large absolute values correspond to variables with greater discriminating power. Hair, et al suggest a threshold of .40 for significance when discerning variables with substantial contributory ability. A review of the standardized discriminant function coefficients (Table 2.6.5) suggests that COMPANY is the most discriminating variable on the first function at .786, followed by SUPERVISOR at .560. The interaction effects of QUALITY of WORK-LIFE and OPPORTUNITY play the primary discriminatory role for the second function.

\begin{tabular}{|l|c|c|}
\hline \multicolumn{3}{|c|}{$\begin{array}{c}\text { Standardized Canonical Discriminant Function } \\
\text { Coefficients }\end{array}$} \\
\hline & 1 & 2 \\
\hline SUnction \\
\hline COMP & .560 & -.013 \\
\hline OPPTY & .786 & -.365 \\
\hline QWL & -.213 & -.311 \\
\hline SUPV*CO & -.218 & .026 \\
\hline COMP*QWL & .329 & .325 \\
\hline QWL*OPPTY & -.202 & -.169 \\
\hline
\end{tabular}

Table 2.6.5 Discriminant function coefficients 
It is also worthwhile to consider the structure matrix which provides the correlation of each predictor variable with the discriminant function (Table 2.6.6).

\begin{tabular}{|l|l|l|}
\hline Structure Matrix \\
\hline & Function \\
\hline & 1 & 2 \\
\hline COMP & .710 & -.400 \\
\hline SUPV & .455 & -.019 \\
\hline QWL & -.197 & .014 \\
\hline SUPV*OPPTY & .042 & -.023 \\
\hline QWL*OPPTY & .182 & .786 \\
\hline OPPTY & -.190 & -.535 \\
\hline SUPV*CO & .211 & .342 \\
\hline COMP*QWL & -.112 & -.253 \\
\hline COMP*OPPTY & -.017 & -.129 \\
\hline SUPV*QWL & -.040 & -.089 \\
\hline $\begin{array}{l}\text { Table 2.6.6 Structure matrix, correlation of factors and interaction } \\
\text { variables with function }\end{array}$ &
\end{tabular}

Intuitively, it makes sense that an employee's perception of stability is initially impacted by the company itself, followed by one's relationship with a supervisor. Given the states of these two factors, or the first function, it then follows that the employee's perceptions of external influences (QWL*OPPTY) can serve as a catalyst to transition states, particularly if the individual is highly influenced by potential outside opportunities.

The stepwise results add insight to this intuition (Table 2.6.7) by clarifying the impact of each variable in the analysis on separating specific groups. For example, the COMPANY factor (sometimes referred to here as a variable or variate, but recall that each of these are actually factors comprised of scores from multiple variables) clearly differentiates non-seekers from passives. In effect, if the employee is highly satisfied with the company (proud to work there, thinks the company is honest, provides valuable products and services, etc.), he/she is more likely to be a non-seeker (group 3). When the score for COMPANY erodes, a transition to the passive state (group 2) is likely to follow. The same holds true for QWL*OPPTY; as these elements become more attractive, more influential, non-seekers may begin to consider seeking. 


\begin{tabular}{|c|c|c|c|c|c|c|c|}
\hline \multicolumn{8}{|c|}{ STEPWISE analysis - Variables Entered/Removed ${ }^{\mathrm{a}, \mathrm{b}, \mathrm{c}}$, } \\
\hline \multirow[t]{3}{*}{ Step } & \multirow[t]{3}{*}{ Entered } & \multicolumn{6}{|c|}{ Min. D Squared } \\
\hline & & \multirow[t]{2}{*}{ Statistic } & \multirow{2}{*}{$\begin{array}{l}\text { Between } \\
\text { Groups }\end{array}$} & \multicolumn{4}{|l|}{ Exact $\mathrm{F}$} \\
\hline & & & & Statistic & df1 & $\mathrm{df} 2$ & Sig. \\
\hline 1 & COMP & .152 & 2.00 and 3.00 & 7.967 & 1 & 1105 & .005 \\
\hline 2 & QWL*OPPTY & .500 & 2.00 and 3.00 & 13.075 & 2 & 1104 & $2.442 \mathrm{E}-6$ \\
\hline 3 & SUPV & .640 & 2.00 and 3.00 & 11.135 & 3 & 1103 & $3.340 \mathrm{E}-7$ \\
\hline 4 & SUPV*CO & .831 & 2.00 and 3.00 & 10.829 & 4 & 1102 & $1.298 \mathrm{E}-8$ \\
\hline 5 & COMP*QWL & .860 & 1.00 and 2.00 & 35.583 & 5 & 1101 & 7.930E-34 \\
\hline 6 & QWL & .902 & 1.00 and 2.00 & 31.068 & 6 & 1100 & $1.321 \mathrm{E}-34$ \\
\hline 7 & OPPTY & .919 & 1.00 and 2.00 & 27.118 & 7 & 1099 & $1.740 \mathrm{E}-34$ \\
\hline \multicolumn{8}{|c|}{$\begin{array}{l}\text { At each step, the variable that maximizes the Mahalanobis distance between the two closest groups } \\
\text { is entered. }\end{array}$} \\
\hline \multicolumn{8}{|c|}{ a. Maximum number of steps is 20 . } \\
\hline \multicolumn{8}{|c|}{ b. Maximum significance of $\mathrm{F}$ to enter is .05 . } \\
\hline \multicolumn{4}{|c|}{ c. Minimum significance of $\mathrm{F}$ to remove is .10 . } & & & & \\
\hline
\end{tabular}

Table 2.6.7 Stepwise results -7 variables in the final discriminant function

\section{Group Differences on Discriminant Scores}

To assess significant differences between the discriminant scores for classes of job-seeking status, t-tests for significance of differences between the means of two groups were conducted (Table 2.6.8 and Table 2.6.9). In all cases significant differences were identified between the mean discriminant scores of each group, as determined by functions 1 and 2 . 


\begin{tabular}{|c|c|c|c|c|c|c|}
\hline & $\begin{array}{l}\text { Seekers } \\
\text { Group } 1 \\
\text { n=1051 }\end{array}$ & $\begin{array}{l}\text { Passives } \\
\text { Group } 2 \\
n=410\end{array}$ & T Stat & $\begin{array}{l}\text { Sig. } \\
\text { tailed }\end{array}$ & 2 & Mean diff \\
\hline $\begin{array}{l}\text { Discriminant scores } \\
\text { Function } 1\end{array}$ & -.332 & .535 & -15.31 & .000 & & -.867 \\
\hline $\begin{array}{l}\text { Discriminant scores } \\
\text { Function } 2\end{array}$ & .024 & -.170 & 3.46 & .001 & & .194 \\
\hline
\end{tabular}

Table 2.6.8 Significant differences - mean discriminant scores for seekers vs. passives

\begin{tabular}{|lllllll|} 
& $\begin{array}{l}\text { Passives } \\
\text { Group 2 } \\
\mathbf{n = 4 1 0}\end{array}$ & $\begin{array}{l}\text { Non-seekers } \\
\text { Group } 3 \\
\mathbf{n = 9 3}\end{array}$ & T Stat & $\begin{array}{l}\text { Sig. } \\
\text { tailed }\end{array}$ & 2 & Mean diff \\
\hline $\begin{array}{l}\text { Discriminant scores } \\
\text { Function 1 }\end{array}$ & .535 & 1.43 & -7.61 & .000 & -.897 \\
\hline $\begin{array}{l}\text { Discriminant scores } \\
\text { Function 2 }\end{array}$ & -.170 & .369 & -2.61 & .010 & .53 \\
\hline
\end{tabular}

Table 2.6.9 Significant differences - mean discriminant scores for passives vs. non- seekers

\section{Classification and Assessment of Accuracy}

Once a set of $q$ variables has been selected, as in the stepwise results above (Table 2.6.7), the classification function (Fisher's linear discriminant function) is computed as:

$b_{i j}=(n-g) \sum_{l=1}^{q} w_{i l}^{*} \bar{x}_{l j} \quad i=1,2, \ldots, q \quad j=1,2, \ldots, \mathrm{g}$ for the coefficients, and $a_{j}=\log p_{j}-\frac{1}{2} \sum_{i=1}^{q} b_{i j} \bar{x}_{i j} \quad j=1,2, \ldots, q$ for the constant,

where $p_{j}$ is the prior probability of group $j$.

Table 2.6.10 provides the classification function coefficients used to assign cases to groups of seekers, passives and non-seekers. There is a separate function for each group. For each case, a classification score is computed for each function and each case is assigned by the model to the group whose classification function obtains the highest score. 


\begin{tabular}{|l|l|l|l|}
\hline \multicolumn{4}{|l|}{ Classification Function Coefficients } \\
\hline & \multicolumn{2}{l}{ Seeker 1, Passive 2 } \\
Non-seeker 3
\end{tabular}

Table 2.6.10 Fisher's linear discriminant functions

To assess classification accuracy for discriminant analysis of the job-seeker data, the estimation sample, a hold-out sample and a cross-validation sample are considered. Moreover, three separate discriminant analyses were run, under the same procedure, yet using a new validation sample each time. The results of these three tests are averaged and discussed.

Table 2.6.11 provides a summary of three tests of validation which provide an average hold-out sample classification rate of $66.4 \%$ for seekers, $48.1 \%$ for passives, and $50.5 \%$ for non-seekers. All classifications based on the most conservative approach, the hold-out sample, surpass the three-group hit-ratio standard of $41.25 \%$ (33\% standard probability for 3 groups $\times 1.25$ as suggested by Hair et al).

In the cross-validation sample, seekers are correctly classified an average of $65.1 \%$ of the time, passives are correctly classified an average of $47 \%$ of the time, and non-seekers were correctly classified an average of $52.9 \%$ of the time, which again all exceed the standard chance criterion of $41.25 \%$.

In the least conservative estimation (or analysis) sample, the results are $65.5 \%$ accuracy for seekers, $47.6 \%$ for passives and 55.5\% for non-seekers. Full results for the classification matrices from the three validation tests (using pooled covariance matrices) are found in Appendix B. 


\begin{tabular}{|c|c|c|c|}
\hline \multicolumn{4}{|c|}{$\begin{array}{l}\text { Percent Average Correct Classification - POOLED } \\
\text { Covariance Matrices }\end{array}$} \\
\hline & Seekers & Passives & $\begin{array}{l}\text { Non- } \\
\text { seekers }\end{array}$ \\
\hline \multicolumn{4}{|c|}{ Estimation Sample } \\
\hline Test 1 & 66.8 & 49.8 & 43.8 \\
\hline Test 2 & 64.0 & 50.2 & 56.5 \\
\hline Test 3 & 65.6 & 42.9 & 66.1 \\
\hline AVERAGE & 65.5 & 47.6 & 55.5 \\
\hline \multicolumn{4}{|c|}{ Cross-validation Sample } \\
\hline Test 1 & 66.8 & 49.5 & 43.8 \\
\hline Test 2 & 63.5 & 48.9 & 53.6 \\
\hline Test 3 & 64.9 & 42.6 & 61.3 \\
\hline AVERAGE & 65.1 & 47.0 & 52.9 \\
\hline \multicolumn{4}{|c|}{ Hold-out Sample } \\
\hline Test 1 & 67.0 & 50.0 & 45.0 \\
\hline Test 2 & 67.8 & 47.3 & 42.4 \\
\hline Test 3 & 64.4 & 46.9 & 64.1 \\
\hline AVERAGE & 66.4 & 48.1 & 50.5 \\
\hline
\end{tabular}

Recall the earlier discussion regarding the significance of Box's M, suggesting the use of separate covariance matrices (quadratic linear analysis). Although liberties are taken with the assumption of equal covariance matrices, an attempt is made to justify classification based on pooled covariance matrices discussed above.

Minimal change occurs by using separate covariance matrices (Table 2.6.12) with only slight deterioration of classification accuracy, primarily in the passive category with an erosion of $3.3 \%$ within the conservative hold-out sample. Accuracy deteriorates only 1.3\% for seekers and $2 \%$ for non-seekers based on the hold-out sample. Comparing the estimation samples, separate covariance matrices perform slightly better for seekers and passives, yet substantially worse (by 10\%) in the non-seeker category. In all analyses, and for each category, the model performs substantially better than chance. 


\begin{tabular}{|c|c|c|c|}
\hline \multicolumn{4}{|c|}{$\begin{array}{l}\text { Percent Average Correct Classification - SEPARATE } \\
\text { Covariance Matrices }\end{array}$} \\
\hline & Seekers & Passives & $\begin{array}{l}\text { Non- } \\
\text { seekers }\end{array}$ \\
\hline \multicolumn{4}{|c|}{ Estimation Sample } \\
\hline Test 1 & 66.8 & 59.5 & 26.6 \\
\hline Test 2 & 66.9 & 37.7 & 61.9 \\
\hline Test 3 & 63.4 & 50.9 & 47.9 \\
\hline AVERAGE & 65.7 & 49.3 & 45.5 \\
\hline \multicolumn{4}{|c|}{ Hold-out Sample } \\
\hline Test 1 & 63.8 & 53.2 & 31.0 \\
\hline Test 2 & 67.3 & 38.1 & 60.0 \\
\hline Test 3 & 64.3 & 43.0 & 54.5 \\
\hline AVERAGE & 65.1 & 44.8 & 48.5 \\
\hline
\end{tabular}

Table 2.6.12 Average correct classifications from 3 validation tests

Separate covariance matrices

Press' $Q$

A final measure of classification accuracy, Press' $Q$, is more statistical in nature as it tests the discriminatory power of the classification matrix when compared to the chance model. The measure compares the number of correct classifications with the total sample size and the number of groups. This value is then compared with a critical chi-square value for 1 degree of freedom, with selected confidence, say .95. If the statistic exceeds the critical value (e.g. 6.68 for $95 \%$ confidence with 1 degree of freedom), then the classification matrix is considered statistically better than chance (Hair et al, p. 268).

$$
Q=\frac{\left.\left\ulcorner N-n_{\text {corr }} g\right)\right]^{2}}{N(g-)}
$$

where

$$
\begin{aligned}
& N=\text { Total sample size } \\
& \mathrm{n}_{\text {corr }}=\text { number of correctly classified observations } \\
& \mathrm{g}=\text { number of groups }
\end{aligned}
$$

To illustrate validity, consider the classification matrix derived from the discriminant analysis procedure described throughout this section (Table 2.6.13). Recall that validation tests were conducted three times and an average reported. Following is the classification matrix using the second validation set as the hold-out sample. 


\begin{tabular}{|c|c|c|c|c|c|c|c|}
\hline \multicolumn{8}{|c|}{ Classification Results ${ }^{a, b, c, d}$} \\
\hline & & & \multirow{2}{*}{$\begin{array}{l}\text { True } \\
\text { Grp }\end{array}$} & \multicolumn{4}{|c|}{ Predicted Group Membership } \\
\hline & & & & 1 & 2 & 3 & Total \\
\hline \multirow[t]{12}{*}{ Cases Selected } & \multirow[t]{6}{*}{ Original } & \multirow[t]{3}{*}{ Count } & 1 & 517 & 188 & 53 & 758 \\
\hline & & & 2 & 86 & 127 & 73 & 286 \\
\hline & & & 3 & 3 & 24 & 37 & 64 \\
\hline & & \multirow[t]{3}{*}{$\%$} & 1 & 68.2 & 24.8 & 7.0 & 100 \\
\hline & & & 2 & 30.1 & 44.4 & 25.5 & 100 \\
\hline & & & 3 & 4.7 & 37.5 & 57.8 & 100 \\
\hline & \multirow[t]{6}{*}{ Cross-validated $^{\mathrm{a}}$} & \multirow[t]{3}{*}{ Count } & 1 & 516 & 188 & 54 & 758 \\
\hline & & & 2 & 86 & 126 & 74 & 286 \\
\hline & & & 3 & 3 & 26 & 35 & 64 \\
\hline & & \multirow[t]{3}{*}{$\%$} & 1 & 68.1 & 24.8 & 7.1 & 100 \\
\hline & & & 2 & 30.1 & 44.1 & 25.9 & 100 \\
\hline & & & 3 & 4.7 & 40.6 & 54.7 & 100 \\
\hline \multirow[t]{6}{*}{ Cases Not Selected } & \multirow[t]{6}{*}{ Original } & \multirow[t]{3}{*}{ Count } & 1 & 196 & 79 & 18 & 293 \\
\hline & & & 2 & 49 & 50 & 25 & 124 \\
\hline & & & 3 & 3 & 12 & 14 & 29 \\
\hline & & \multirow[t]{3}{*}{$\%$} & 1 & 66.9 & 27.0 & 6.1 & 100 \\
\hline & & & 2 & 39.5 & 40.3 & 20.2 & 100 \\
\hline & & & 3 & 10.3 & 41.4 & 48.3 & 100 \\
\hline \multicolumn{8}{|c|}{$\begin{array}{l}\text { a. Cross validation is done only for those cases in the analysis. In cross validation, each case is classifie } \\
\text { by the functions derived from all cases other than that case. }\end{array}$} \\
\hline \multicolumn{8}{|c|}{ b. $61.5 \%$ of selected original grouped cases correctly classified. } \\
\hline \multicolumn{8}{|c|}{ c. $58.3 \%$ of unselected original grouped cases correctly classified. } \\
\hline \multicolumn{6}{|c|}{ d. $61.1 \%$ of selected cross-validated grouped cases correctly classified. } & & \\
\hline
\end{tabular}

Table 2.6.13 Classification matrix validated with Press' Q statistic

The Press' $Q$ statistics for the classification matrix discussed here are $Q=$ 641.86 for the estimation sample, $Q=419.46$ for the cross-validation sample and $Q=214.53$ for the hold-out sample. All far exceed the critical value of 6.68 , suggesting a general sensitivity to large sample sizes which are more likely to evidence statistical significance for overall classification rates.

Below, Figure 2.6.3 depicts the amount of discrimination provided by the first discriminant function, based on the discriminant scores. While it is still challenging to discern seeking status based on the discriminant scores, substantial separation of the densities is evident. This first discriminant function accounts for approximately $95 \%$ of the discrimination with the second contributing only $5 \%$ (Figure 2.6.4). Thus, the balance of the discussion will cover only the first function. 
Predicted Group Membership - Discriminant Function 1

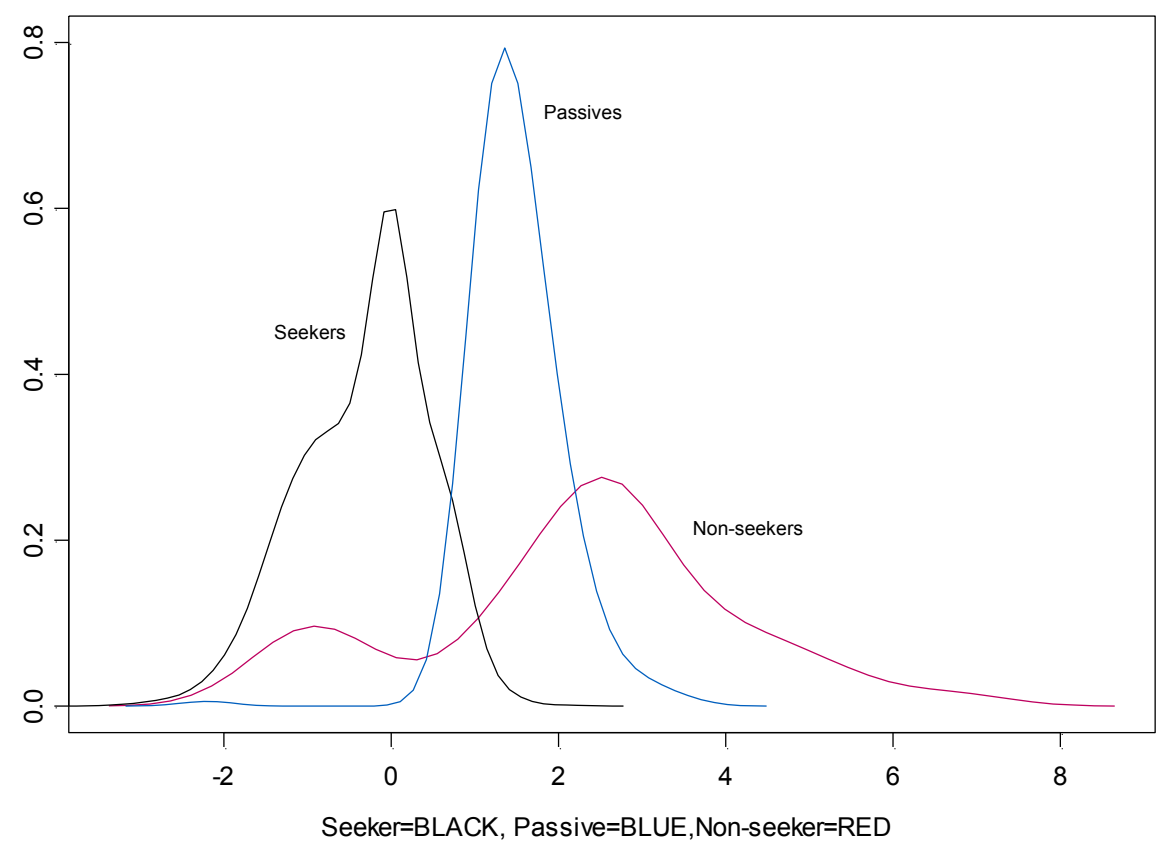

Figure 2.6.3 Separation produced by first discriminant function

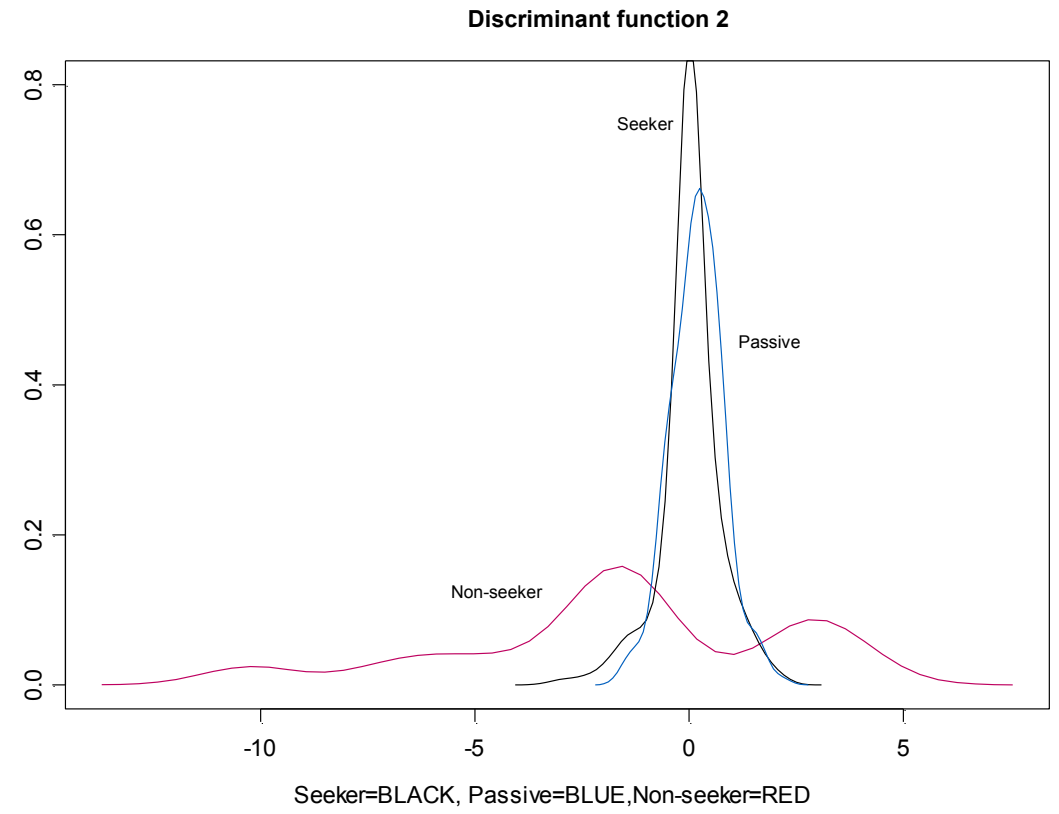

Figure 2.6.4 Separation produced by second discriminant function

A straightforward validation of the first function's discriminatory power is simply the descriptive statistics for the discriminant scores for each job- 
seeking category (Tables 2.6.14 to Table 2.6.16). Seekers exhibit a mean of .31 , passives 1.5 and non-seekers 2.2 .

Seekers

\begin{tabular}{|l|l|l|l|l|l|}
\hline Descriptive Statistics & $\mathrm{N}$ & Minimum & Maximum & Mean & Std. Deviation \\
\hline $\begin{array}{l}\text { Discriminant Scores from } \\
\text { Function 1 for Analysis 1 }\end{array}$ & 1555 & -3.21 & 2.09 & -.31 & .754 \\
\hline
\end{tabular}

Table 2.6.14 Seeker category discriminant score descriptive statistics

Passives

\begin{tabular}{|l|l|l|l|l|l|}
\hline Descriptive Statistics & N & Minimum & Maximum & Mean & Std. Deviation \\
\hline $\begin{array}{l}\text { Discriminant Scores from } \\
\text { Function 1 for Analysis 1 }\end{array}$ & 218 & -2.19 & 3.51 & 1.5 & .517 \\
\hline
\end{tabular}

Table 2.6.15 Passive category discriminant score descriptive statistics

Non-seekers

Descriptive Statistics

\begin{tabular}{|l|l|l|l|l|l|}
\hline & $\mathrm{N}$ & Minimum & Maximum & Mean & Std. Deviation \\
\hline $\begin{array}{l}\text { Discriminant Scores from } \\
\text { Function 1 for Analysis 1 }\end{array}$ & 44 & -1.46789 & 6.74421 & 2.2 & 1.89 \\
\hline
\end{tabular}

Table 2.6.16 Non-seekers category discriminant score descriptive statistics

Testing for significant differences between the discriminant score means of each of the job-seeking categories shows that all categories evidence significant differences. Between seekers (-.31) and passives (1.5), the mean difference between discriminant scores is 1.81 , with a very substantial $t=-5.39$. Between passives (1.5) and non-seekers (2.2) the mean difference is -.72 , with $t=-.51$

While the second function does not offer much graphic stimulation as evidenced by Figure 2.6.6, it does still serve to deliver another five percent discrimination, and there are significant differences between the mean discriminant scores for each category. Between seekers and passives, the mean difference is .15 and $t=-3.08$. Between passives and non-seekers, the mean difference for the second discriminant function .18 and $t=3.2$, still significant at $p<.01$. 


\section{Discussion}

Discriminant analysis techniques have allowed a reasonably accurate classification of job-seeking status, well exceeding chance. As expected, the most challenging group to classify is the passive employees even though significant differences are identified between this group versus seekers, and versus non-seekers.

Utilizing the stepwise procedure, plus subsequent investigation of the structure matrix (examining the correlation between variables and the discriminant function), suggests the most discriminatory constructs of satisfaction and influence to be COMPANY, followed by the interaction of QUALTIY of WORK-LIFE * OPPORTUNITY, and finally SUPERVISOR. All three are helpful in discriminating between passives and non-seekers. 


\subsection{Building a Generalized Additive Model to Predict Job-Seeking Status}

Reasoning and Goal

In section 1.4 the general problem of classifying job-seeking status was discussed as were some of the limitations related to the objective measurement of loyalty. Regression models were briefly considered as a data analytic tool for understanding the impact of different inputs on loyalty. As clarified in Sections 2.2 and 2.3, the job-seeking status data is clearly not normally distributed. Models such as the normal linear regression model are often used for prediction and classification rules, yet these traditional linear models often fail in application because in real life effects are often not linear (Hastie, Tibshirani \& Friedman, 2001). Thus, a modeling method is sought that best handles non-Gaussian data, with multiple covariates, in a manner that enables the prediction of categorical data as in the job-seeking status case.

Generalized additive models (GAM) hold promise for marketing applications, particularly because of the relaxation of linearity constraints, yet their use in management and marketing contexts has been limited. Abe (1999) used the GAM framework in a multinomial discrete-choice context to identify threshold and saturation effects of a price promotion. This is particularly useful to marketers who typically set prices and determine promotional plans. Berg (2007) used GAM in a financial context to predict bankruptcy, simultaneously showing that the GAM outperformed linear discriminant analysis.

Very recently, researchers have shown how GAM can improve marketing decision making in a customer churn context (Coussement, Benoit, Van den Poel, 2010). The identification of risky customers, or employees in the jobseeking context, coupled with increased interpretability of existing models with graphics, offers managers and marketers a valuable decision making tool.

Generalized Additive Models

Generally speaking, generalized additive models can uncover predictorresponse relationships in varied data structures without the requirement of a specific model (Cai, 2008). Stone (1985) proposed additive models to estimate an additive approximation to the multivariate regression function. Univariate smoothers are used, and estimates of the individual terms explain how the response variable changes with corresponding changes in the independent variables. The additive model has the form 


$$
E(Y \mid \mathbf{X})=\sum_{1}^{p} s_{j}\left(X_{j}\right)
$$

Two important extensions were developed from the additive model. Friedman and Stuetzle (1981) proposed the projection pursuit regression model:

$$
E(Y \mid \mathbf{X})=\sum^{p} s_{j}(\alpha \quad r)
$$

where numerical search is used to find the directional vectors, $\mathbf{a}_{\mathrm{j}}$, while the $s_{j}(\cdot$ ''s are estimated by smoothers.

Breiman and Friedman (1985) introduced a generalization of the additive model by estimating a transformation of the response variable. The alternating conditional expectation (ACE) model:

$$
E(\theta Y) \mid \mathbf{X})=\sum_{1}^{p} s_{j}\left(X_{j}\right)
$$

Hastie and Tibshirani $(1986,1987)$ proposed generalized additive models as an alternative to likelihood-based regression models (e.g., normal linear, linear logistic) and other parametric models. Generalized additive models replace the linear form $\sum \beta, X_{j}$ by a sum of smooth functions $\sum s_{j}\left(X_{j}\right)$. The $s_{j}(\cdot$ 's are unspecified smooth functions, estimated with a scatterplot smoother. An algorithm is developed to simultaneously estimate all functions.

Hastie et al (2001) suggest easily mixing linear and other parametric forms with the nonlinear terms. This is essential when some of the inputs are qualitative variables. Nonlinear components in two or more variables, and separate curves in the $X_{\mathrm{j}}$ for each level of the factor $X_{\mathrm{k}}$ are acceptable, adding to the flexibility of the generalized additive model (GAM). The GAM can be used to identify and characterize nonlinear regression effects. Such models assume that the mean of the dependent variable depends on each of the additive predictors via a nonlinear link function. The probability distribution of the response variable can represent any member of the exponential family of distributions. In general, the conditional mean $\mu(\mathrm{X})$ of a response $Y$ is related to an additive function of the predictors via a link function $g$ :

$$
g[\mu x)]=\alpha-f_{1}\left(X_{1}\right)+. .+f_{p}\left(X_{p}\right) .
$$

The functions $f_{j}$ can be flexibly estimated using an algorithm based on the scatterplot smoother, and can thus reveal nonlinearities in the effect of $X_{j}$. 
Gentle (2002, p.304) explains that the appropriate form of the link function depends on the nature of the probability distribution, and Hastie et al (2001, p.258) identify examples of three classical link functions including:

- $g(\mu)=\imath$ is the identity link, used for linear and additive models for Gaussian response data

- $g(\mu=\operatorname{og}(\mu$ for log-linear or log-additive models for Poisson data

- $g(\mu=\operatorname{logit}(\mu)$ for two-class classification where the mean of the binary response $\mu X)=\operatorname{s} r(Y=\mid X)$ is related to the predictors via a linear regression model and the logit link function:

$$
g\left(\mu=\operatorname{sg}\left(\frac{\mu X)}{-\mu X)}\right)=\alpha \cdot f_{1}\left(X_{1}\right)+. f_{p}\left(X_{p}\right) .\right.
$$

For more than two classes, as in the job-seeker data, the additive logistic regression model can be generalized further using a multinomial logit formulation as will be discussed in the application that follows.

Fitting an Additive Model

As mentioned earlier, the $f_{j}$ 's are unspecified smooth nonparametric functions which can each be fit using a scatterplot smoother. Then one subsequently develops an algorithm for simultaneously estimating all $p$ functions. Hastie et al (2001, p. 260) suggest using cubic smoothing splines.

In a discussion on splines, Gentle (2002, p. 139) first recalls that a smooth approximation, $\hat{f}(x)$, is one that is continuous with continuous derivatives. The polynomials in $\hat{f}(x)$ cause undesirable oscillations, and if the function being estimated has different shapes in different regions of its domain, using the same polynomial over the full domain may not be accurate. The interval over which the function is being approximated can be subdivided, and a polynomial with a low degree can be used. Then, the piecewise polynomials can be summed to approximate any point. Using more and more subintervals may be impractical and complicated, so continuity restrictions are imposed on the piecewise polynomials and their derivatives. This describes the spline approach to approximation and smoothing.

In spline approximation, the basis functions are polynomials over given intervals and zero outside of those intervals, with specified contacts or "knots" at the endpoints which are their continuous derivatives of a specified order. The approximation $\hat{f}(x)$ is then formed as the sum of the piecewise polynomials, and this is the spline. The number of free parameters in each 
interval is called the "order" of the spline. A common spline basis function is the natural polynomial spline in which the second derivative of the spline expansion is 0 for all $x$ beyond the boundary knots. For natural cubic splines with $k$ knots, there are $k$ basis functions.

Hastie et al (2001, p. 259) recall the additive model with the form

$$
Y=\alpha \cdot \sum_{j=1}^{p} f_{j}\left(X_{j}\right)+\varepsilon
$$

where the error term has a mean zero. Given observations $x_{i} y_{i}$ the penalized sum of squares criterion is specified where

$$
\left.\operatorname{PRSS}\left(\alpha f_{1}, f_{2}, \ldots, f_{p}\right)=\sum_{i=}^{\prime \prime}\left\{i_{i}-\alpha \cdot \sum_{j=}^{-}\left(x_{i j}\right)\right\}^{-}+\sum_{j=}^{-} \lambda \int f^{\prime}, t_{j}\right)^{2} d t_{j},
$$

where the $\lambda \geq 1$ are tuning parameters. The minimizer of this is an additive cubic spline model and each of the $f_{j}$ functions is a cubic spline in the component $X_{j}$ with knots at each of the unique values of $x_{i j}, i=1, \ldots, N$. Without further restrictions, the model is not unique, so the standard convention is to assume that $\sum_{1}^{N} f_{j}\left(x_{i j}\right)=0 \forall j$, the functions average 0 over the data.

An iterative procedure for finding the solution is then suggested. Set $\alpha=\imath v e\left(y_{i}\right)$ which never changes. Apply a cubic smoothing spline $S_{k}$ (as does Yee in the VGAM procedure in $\mathrm{R}$ ) to the targets

$$
\left\{y_{i}-\alpha-\sum_{j \neq k} \hat{f}_{j}\left(x_{i j}\right)\right\}_{1}^{N}
$$

as a function of $x_{i k}$ to obtain a new estimate, $\hat{f}_{k}$. Do this for each predictor in turn, using the current estimates of the other functions, $\hat{f}_{j}$ when computing

$$
y_{i}-\alpha \cdot \sum_{j \neq k} \hat{f}_{j}\left(x_{i j}\right)
$$

This process is called "backfitting" and the resulting fit is analogous to multiple regression for linear models (Friedman and Stuetzle, 1981). 


\section{Applying Non-parametric Multinomial Regression to Job-Seeker Data}

The goal is to predict whether a subject is a seeker, passive or non-seeker. We can determine the probability with which the subject falls into each of these three seeking-status categories. The additive components are SUPV, COMP, OPPTY, and QWL. The VGAM procedure in $\mathrm{R}$ for generalized additive models uses the logit procedure for each of the three probabilities with the constraint that the sum of the probabilities is equal to one. The model for the multinomial logit procedure is

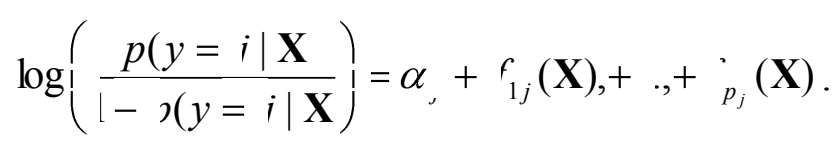

See Yee (2010) for more on the case of a generalized linear model and the logit procedure to be used, or to download the VGAM library. Utilizing the VGAM procedure in $\mathrm{R}$, and calling the family function multinomial (), a fairly flexible model can be fit to the job-seeker data, using three degrees of freedom. A second experiment is discussed in which two degrees of freedom were specified. Figure 2.7.1 shows the estimated functions for each of the predictors. The rug plot along the bottom of the frames indicates the observed values of the corresponding predictor. The discontinuity is evidenced at zero, clarifying nonlinearity, particularly for passives' quality of work-life rating. The $\mathrm{u}$ shaped trend suggests seeking probability decreases until it reaches a minimum. When the values for QWL become larger the chances of seeking are greater.
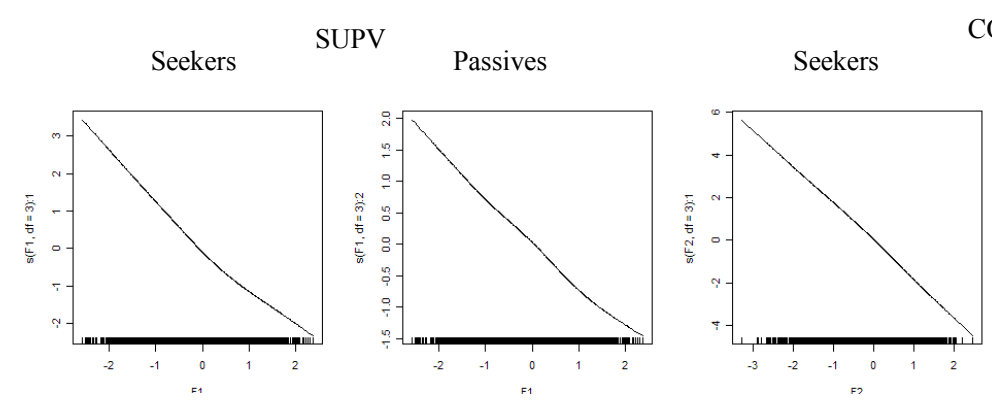

COMP
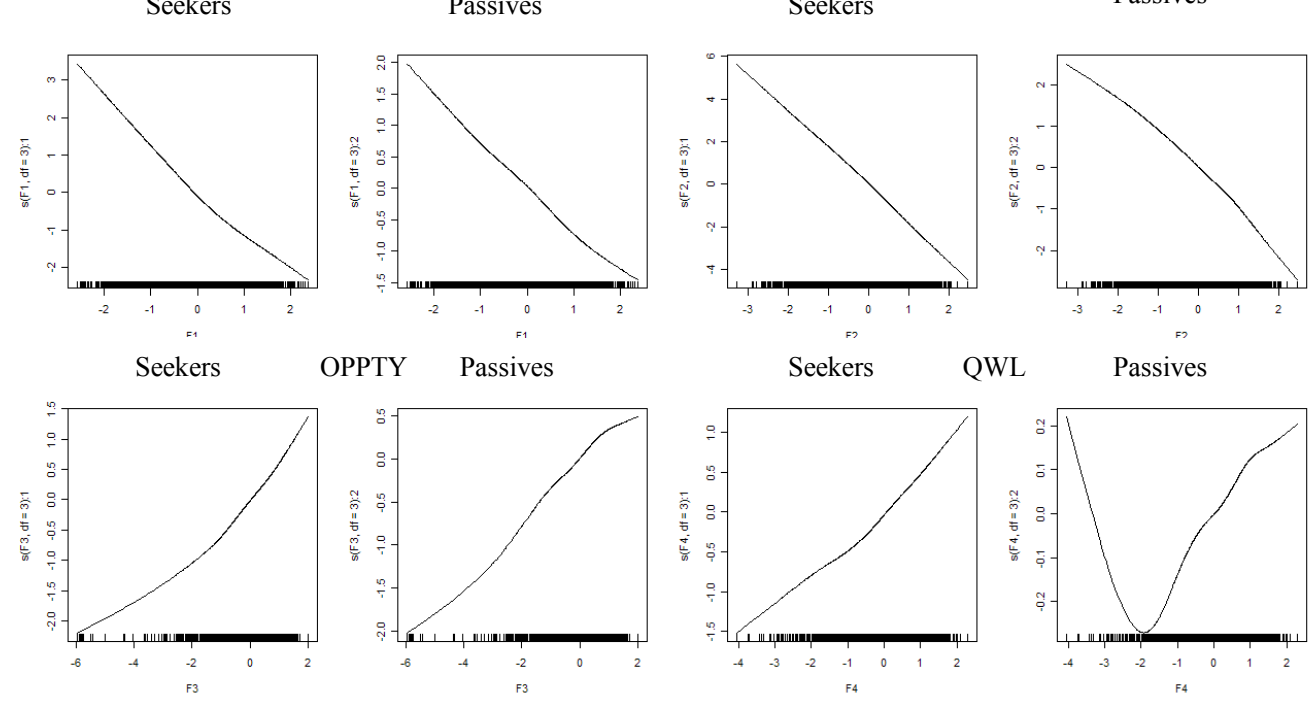

OPPTY Passives

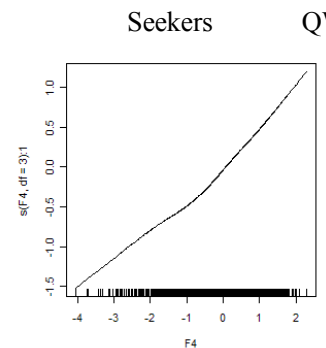

QWL Passives
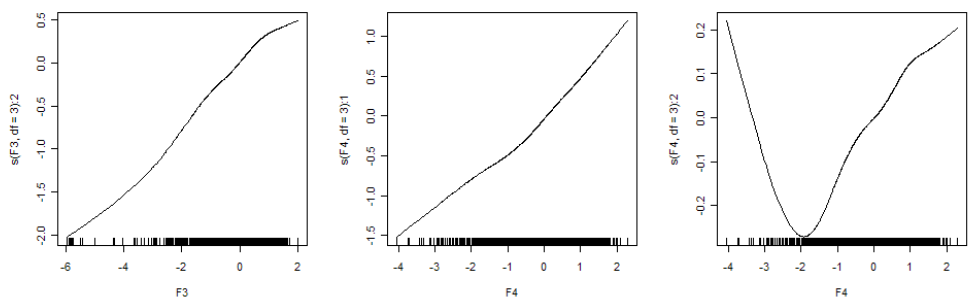

Figure 2.7.1 Functions at three degrees of freedom 
For prediction, vary each of the four variables while holding the others constant. In this way, the prediction function is determined by the model (Figure 2.7.2).
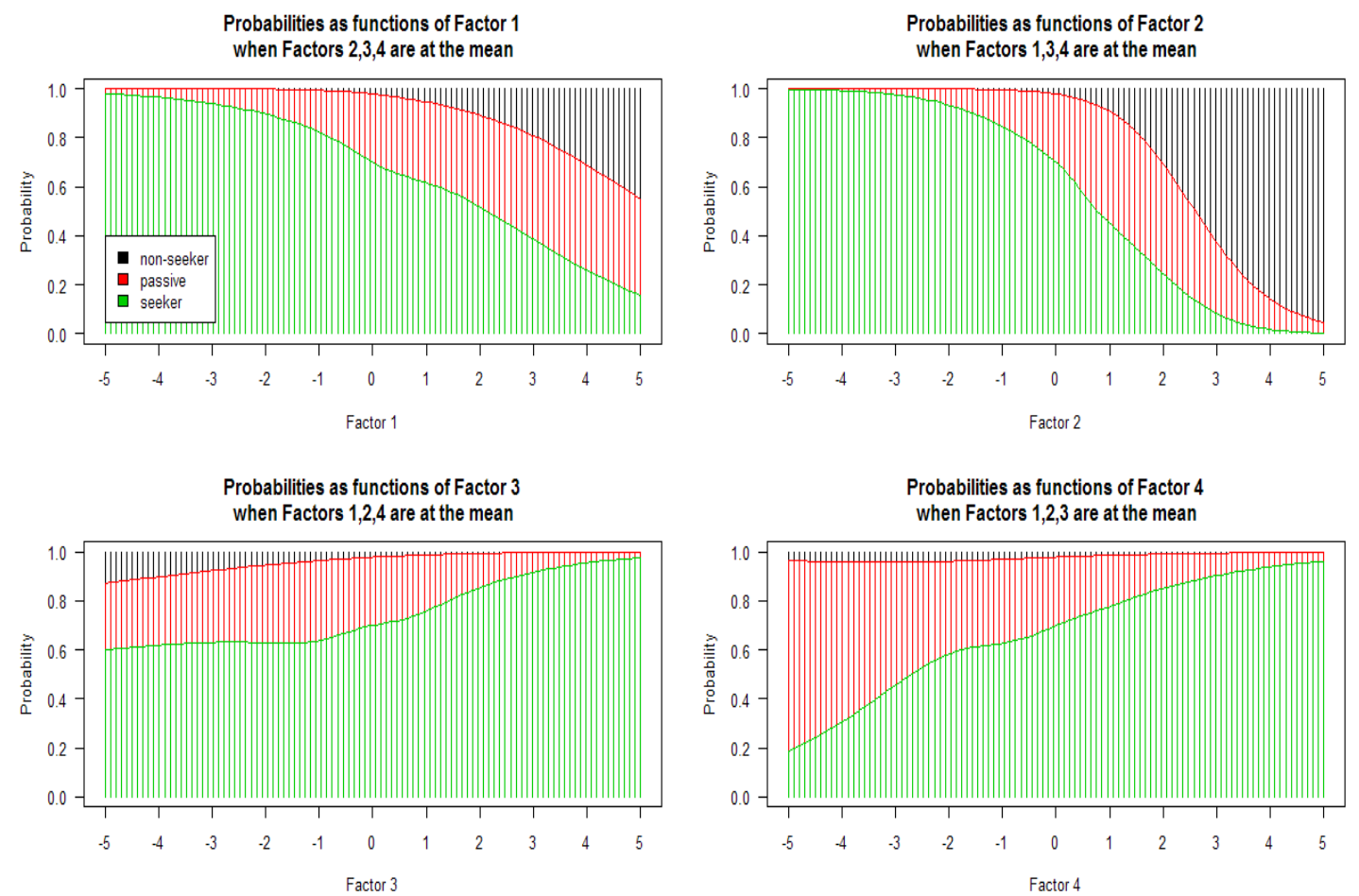

Figure 2.7.2 Varying each factor while holding others constant; three degrees of freedom

Results

Probability of seeking decreases as ratings for supervisor (factor 1, SUPV), increase. There is a fairly steep slope at the mean with a steady decrease in the likelihood of seeking as satisfaction with SUPV increases. The negative slopes separating seekers from passives, and passives from non-seekers are similar on the COMP factor 2, yet more dramatically separating passives from nonseekers as satisfaction with the company increases.

External opportunities (factor 3, OPPTY), including advancement, training, higher salary and better benefits are clearly not as influential to non-seekers. This is evident in that the non-seekers are primarily found below the mean zero, with only minor representation between zero and one. The probability of being a seeker increases steadily, but not dramatically, when external influences, or attraction/expected utility of alternatives (Mobley et al, 1979), increases. External influences related to quality of work-life (factor 4, QWL) have a fairly dramatic effect on separating seekers from passives as the influence of a potentially higher quality of work-life become more attractive. 
This suggests that the probability of seeking increases as the influence of variables such as more flexible hours, the chance to work with a new supervisor, or for a potentially better company, increases.

The scores for an individual can be used in the predictive model to determine the probability of the person being in each of the seeking states. As an example, if a person scores 3 on the supervisor factor (F1), 2 on the company factor (F2), 2 on the opportunity factor (F3) and 1 on the quality of work-life factor (F4), we can specify these figures in the predictive model, resulting in an $18 \%$ probability that the subject is seeking a new position, a $26 \%$ probability that the subject is in a passive state, and a $56 \%$ probability that the subject is not seeking a new position. The person is most likely a non-seeker.

Experimenting with two degrees of freedom, the results are $13 \%, 24 \%$ and $63 \%$ respectively, which enhances the probability of non-seeking status (Figures 2.7.3 and 2.7.4). Overall, there is not a substantial visual difference between two and three degrees of freedom.
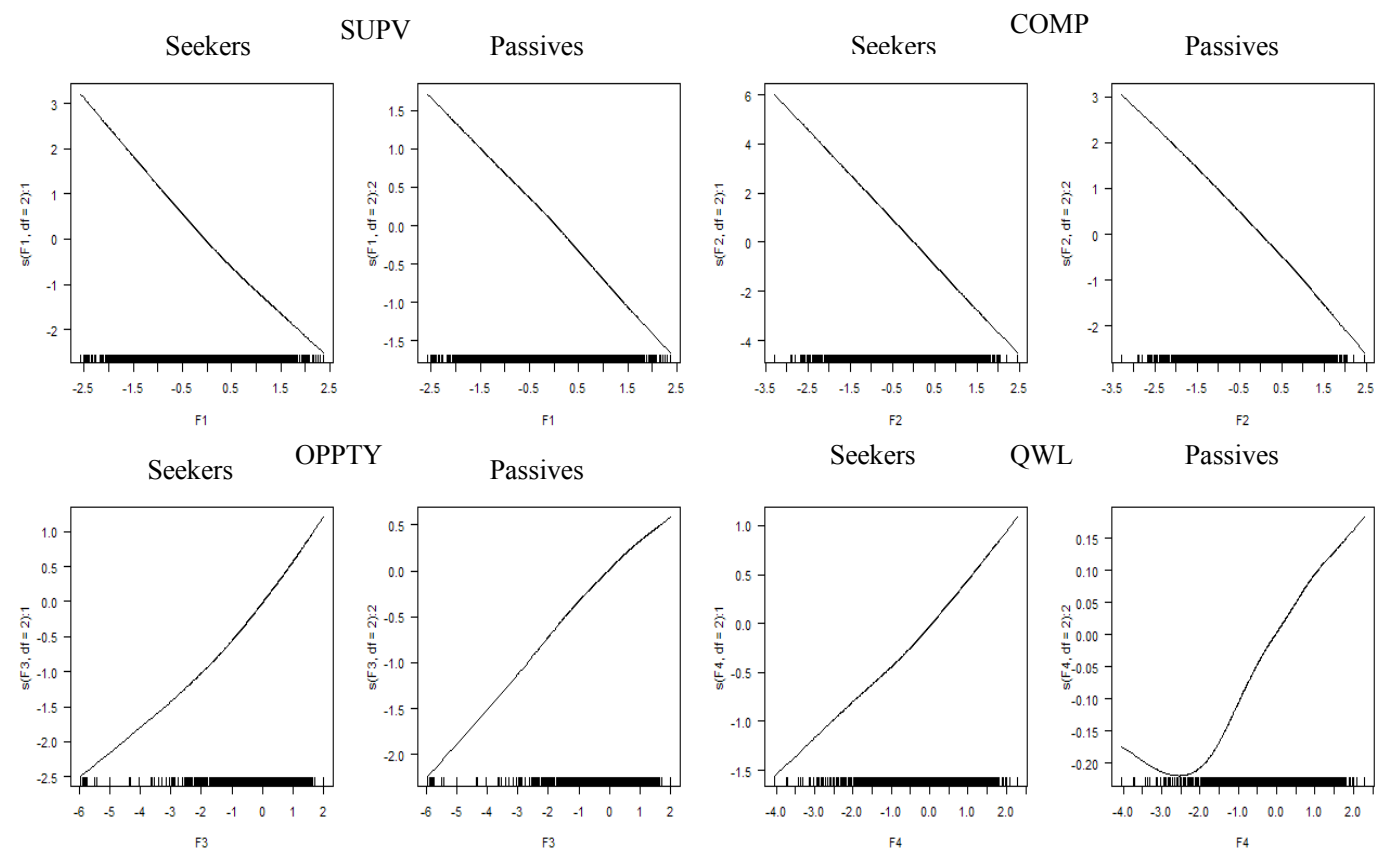

Figure 2.7.3 Functions at two degrees of freedom 

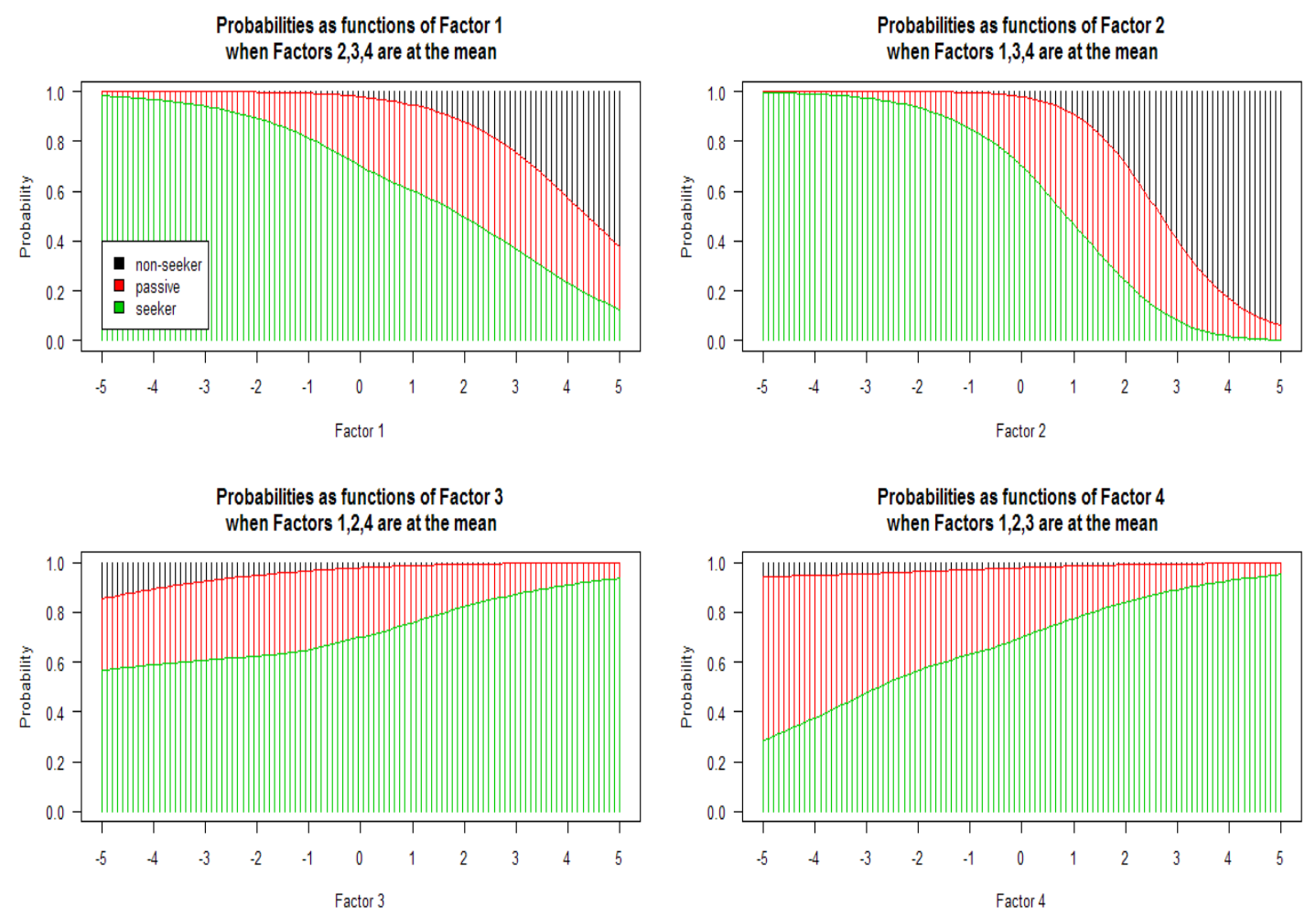

Figure 2.7.4 Varying each factor while holding others constant; two degrees of freedom

\section{Implications}

The generalized additive model provides an effective method for identifying seeking status of an individual employee. Further, the model has confirmed managerial intuitions and extant research regarding the impact of situational variables such as company and supervisor satisfaction as well as external influences related to opportunity and quality of work-life.

The predictive model enables an employer to determine an employee's probability of seeking by indirectly asking a simple set of questions. 


\subsection{Summary, Limitations and Future Research}

The management issue addressed was measuring the stability of an organization with respect to the job-seeking status of its employees. Obtaining the true job-seeking status of an employee is challenging given the risk, or at least perceived risk, involved for the employee. A method has been developed and tested which enables the employer to ask a series of questions related to satisfaction with the company and supervisor, the influences of alternate opportunities, and quality of work-life, and ultimately use the responses to predict the likelihood that the employee is currently seeking a new position, passive, or not seeking.

A loyalty score was developed and its components were discussed thoroughly. An estimation process was detailed, and an application was made using the job-seeker dataset.

The motivation for this portion of the analysis was not only to defend the method used to develop the loyalty score, but also to better understand the underlying factors and how they potentially differ by seeking status. It is clear that significant differences exist between seekers, passives and non-seekers. The loyalty score enabled the identification of the amount of seekers and nonseekers given threshold scores, with $75 \%$ accuracy, but identifying the status of a particular employee would be impractical in a managerial setting.

The more valuable analytical methods used were factor analysis, reducing the variables to four underlying constructs; discriminant analysis to separate groups, and generalized additive models to classify seeking status. Discriminant analysis techniques delivered a reasonably accurate classification of job-seeking status, well exceeding chance. As expected, the most challenging group to classify is the passive employees even though significant differences are identified between this group versus seekers, and versus nonseekers.

A generalized additive model was built and tested which facilitates the classification of employees into seeking categories. The key contribution is that one can specify results based on factor scores in the predictive model, resulting in the probability of the subject falling into each of the three seekingstatus categories. This is achieved without directly asking the employee about their current job-seeking status. Understanding the seeking status of the organization's employee base facilitates an objective measurement of stability. Further, it provides management with the ability to classify relative newcomers based on responses to satisfaction-type questions. 


\section{Limitations and Future Research}

Some of the nineteen questions, related to external influences on job-seeking behaviors, may be more valuable to a loyalty scoring method if posed instead as satisfaction with the elements of one's current employment situation. Further, a response variable such as overall satisfaction with current job or with one's current company, or alternately one's supervisor, would facilitate linear regression more effectively versus using a measurement of "likelihood to recommend the company" as a dependent variable. Perhaps the question for loyalty measurement is "would you recommend your current position". However, any of these options would mean sacrificing measurements for the effects of external influences which is clearly important in the turnover literature (Mobley, 1979; Cater and Buffardi, 2004; Beauregard and Henry, 2009). The tradeoff in linearity is resolved here with nonparametric approaches, the most effective of which was the generalized additive model.

Generalized additive models (specifically the multinomial logit tested here) hold promise for predictive analytics in marketing and management applications where the data is not normally distributed. For example, brand switching behaviors in oligopolies such as wireless communication providers (e.g., three discrete choices, multinomial logit) could potentially be modeled with GAM. These models may also be appropriate for understanding leadership behaviors as related to or predictive of personal happiness. In the College Student Leadership Behaviors Study (next section) a need is identified for a response variable that measures leadership effectiveness. Perhaps the more important and valuable response outcome of leadership is one's personal happiness, self-reported in discrete categories. Resource allocation applications might include modeling franchisor or customer satisfaction, loyalty, or churn propensity, given multiple marketing and management inputs.

The challenge in management and marketing applications will be the communication of methods and results to non-statistical managers. However, managers will typically be very familiar with the variables or factors presented as covariates, and their intuitions can be supported by effective graphics that enable the visualization of non-linear relationships within customer or employee bases. Abe's work (1999) using generalized additive models to identify thresholds and saturation effects of price promotions should be of particular interest to marketers. After conducting effective modeling of the marketing or management issue with generalized additive models, software for an organization could be developed to generate easily-interpreted results. 


\section{References Classification and Analysis of Job Seeking Market}

Abe, M. (1999). A generalized additive model for discrete-choice data. Journal of Business \& Economic Statistics 17 (3), 271-284.

Acquino, K. R., Griffeth, W., Allen, D.G. \& Hom, P. W. (1997). Integrating justice constructs into the turnover process: A test of a referent cognitions model. Academy of Management Journal 40, 1208- 27.

Altman, E. I. (1968). Financial ratios, discriminant analysis and the prediction of corporate bankruptcy. Journal of Finance 23 (4), 589-609.

Arnold, H. J. \& Feldman, D. C. (1982). A multivariate analysis of the determinants of job turnover. Journal of Applied Psychology 67(3), 350-60.

Atchison, T. J. \& Lefferts, E. A. (1972). The prediction of turnover using Herzberg's job satisfaction technique. Personnel Psychology 25, 53-64.

Badaracco, J. L. (1998). The discipline of building character. Harvard Business Review, March/April, 116.

Beauregard, T. A. \& Henry L. C. (2009). Making the link between work-life balance practices and organizational performance. Human Resource Management Review 19, 9-22.

Berg, D. (2007). Bankruptcy prediction by generalized additive models. Applied Stochastic Models in Business and Industry 23 (2), 129-143.

Berkowitz, L., Fraser, C., Treasure, F. P. \& Cochran S. (1987). Pay, equity, job gratifications, and comparisons in pay satisfaction. Journal of Applied Psychology 72, 544-51.

Blau, P. M., Gustad, J. W., Jessor, R., Parnes, H. S. \& Wilcox R. C. (1956). Occupational choice: A conceptual framework. Industrial and Labor Relations Review 8, 531-43.

Box, G. E. P. (1949). A general distribution theory for a class of likelihood criteria. Biometrika 36, 317-46.

Brayfield, A. H. \& Crockett, W. H. (1955). Employee attitudes and employee performance. Psychological Bulletin 52, 396-424.

Brough, P., \& Frame, R. (2004). Predicting police job satisfaction and turnover intentions: The role of social support and police organizational variables. New Zealand Journal of Psychology 1 March. 
Cai, W. (2008). Fitting generalized additive models with GAM procedure in $S A S$ 9.2. Proc. of SAS Global Forum, SAS Institute, Cary, NC.

Calkins, E. V. (1987). Perceptions of the role of a faculty supervisor or mentor at two medical schools. Assessment and Evaluation in Higher Education 12, 202-08.

Casella, G. \& Berger, R. L. (2002). Statistical inference. Australia: Thomson Learning.

Casper, W. J. \& Buffardi, L. C. (2004). Work-life benefits and job pursuit intentions: The role of anticipated organizational support. Journal of Vocational Behavior 65, 391-410.

Chatterjee, S., Hadi, A. S. \& Price, B. (2000). Regression analysis by example. New York: Wiley.

Cochran, W. G. (1964). On the performance of the linear discriminant function. Technometrics 6.2, 179-90.

Cronbach, L. J. (1951). Coefficient alpha and the internal structure of tests. Psychometrika 16, 297-333.

Coussement, K., Benoit, D. F. \& Van del Poel, D. (2010). Improved marketing decision making in a customer churn prediction context using generalized additive models. Expert Systems with Applications 32, 2132-2143.

DeConick, J. B. \& Stilwell, C. D. (2004). Incorporating organizational justice, role states, pay satisfaction and supervisor satisfaction in a model of turnover intentions. Journal of Business Research 57, 225-231.

Discriminant Analysis - QualtricsWiki. (n.d.). Retrieved 6 Aug. 2009 from Qualtrics Web site:

http://www.qualtrics.com/wiki/index.php/Discriminant_Analysis

Discriminant Function Analysis. (n.d). Retrieved 23 Aug. 2009 from StatSoft | STATISTICA, Predictive Analytics, Statistical Analysis Software Web site: http://www.statsoft.com/TEXTBOOK/stdiscan.html

Ehrenberg, R. G. \& Smith, R. S. (1982). Modern labor economics. Glenview, IL: Scott, Foresman.

Eisenberger, R., Stinglhamber, F., Vandenberghe, C., Sucharski, I. \& Rhoades, L. (2002). Perceived supervisor support: Contributions to 
perceived organizational support and employee retention. Journal of Applied Psychology, 87, 565-573.

Ferguson, T. S. (1996). A course in large sample theory. Boca Raton, FL: Chapman and Hall/CRC.

Fiedler, F. E. (1996). Research on leadership selection and training: One view of the future. Administrative Science Quarterly 41, 241-50.

Fields D., Pang M., \& Chiu C. (2000). Distributive and procedural justice as predictors of employee outcomes in Hong Kong. Journal of Organizational Behavior 21, 547-62.

Fishbein, M. (1967). Attitude and prediction of behavior. Readings in attitude theory and measurement. New York: Wiley.

Fisher C. D. \& Gitelson, R. (1983). A meta-analysis of the correlates of role conflict and ambiguity. Journal of Applied Psychology, 68, 320-33.

Folger, R. \& Cropanzano, R. (1998). Organizational justice and human resource management. Los Angeles: Sage.

Folger, R. \& Konovsky M. A. (1989). Effects of procedural and distributive justice on reactions to pay decisions. Academy of Management Journal 32, 115-30.

Friedman, J. H. \& Stuetzle W. (1981). Projection pursuit regression. Journal of the American Statistical Association, 76, 817-823.

Garson, G. D. (2009). Factor Analysis. Retrieved 18 July 2009 from StatNotes, North Carolina State University, Public Administration Program Web site: http://faculty.chass.ncsu.edu/garson/PA765/factor.htm

Garson, G. D. (2009). Regression. Retrieved 22 May 2009from StatNotes, North Carolina State University, Public Administration Program. Web site: http://faculty.chass.ncsu.edu/garson/PA765/regress.htm.

Garson, G. D. (2009). Scales \& Standard Measures. Retrieved 20 May 2009 from StatNotes, North Carolina State University, Public Administration Program Web site: http://faculty.chass.ncsu.edu/garson/PA765/standard.htm

Gentle, J. E. (2002). Elements of computational statistics. New York: Springer. 
Gerhart, B. (1990). Voluntary turnover and alternative job opportunities. Journal of Applied Psychology 75(5), 467-76.

Good L. K., Page, T. J. \& Young, C. E. (1996). Assessing hierarchical differences in job related attitudes and turnover among retail managers. Journal of the Academy of Marketing Science, 24, 148-56.

Green, P. E., Tull, D. \& Allbaum, G. (1988). Research for marketing decisions. Upper Saddle River, NJ: Prentice Hall.

Greenberg, J. (1990). Organizational justice: yesterday, today and tomorrow. Journal of Management 16, 399-432.

Harman, H. H. (1976). Modern factor analysis. Chicago: University of Chicago Press.

Hartigan, J. A. (1975). Clustering algorithms. New York: John Wiley and Sons.

Hastie, T. J. \& Tibshirani R. J. (1990). Generalized additive models. New York: Chapman and Hall.

Hastie, T., Tibshirani, R. \& Friedman, J. (2001). Elements of statistical learning: Data mining, inference, and prediction. New York: Springer.

Hastie, T., \& Tibshirani, R. (1986). Generalized additive models. Statistical Science 3, 297-318.

Herzberg, F. W. (1966). Work and the nature of man. Cleveland, OH: World.

Huberty, C. J., Wisenbaker, J. W. \& Smith J. C. (1987). Assessing predictive accuracy in discriminant analysis. Multivariate Behavioral Research 22 (July), 307-29.

Jackson, S. E. \& Schuler R. S. (1985). A meta-analysis and conceptual critique of research on role ambiguity and role conflict in work settings. Organizational Behavior and Human Decision Processes 36, 16 - 78.

James L. A. \& James L. R. (1989). Integrating work environment perceptions: explorations into the measurement of meaning. Journal of Applied Psychology 74, 739- 51.

Johnson, R. A. \& Wichern. D. W. (2001). Applied multivariate statistical analysis. 5th ed., Upper Saddle River, NJ: Prentice Hall.

Judd, C. M. \& McClelland, G. H. (1989). Data analysis: A model comparison approach. New York: Harcourt Brace Jovanovich. 
Kaiser, H. F. (1958). The varimax criterion for analytic rotation in factor analysis. Psychometrika 23 187-200.

Keiningham, T. L., Cooil, B., Andreassen, T. W. \& Askoy, L. (2007). A longitudinal examination of net promoter and firm revenue growth. Journal of Marketing 71, 39-51.

Konovsky, M. A., Cropanzano R. (1991). Perceived fairness of employee drug testing as a predictor of employee attitudes and job performance. Journal of Applied Psychology 76, 698-707.

Levene, H. Robust tests for equality of variances. (1960). Contributions to probability and statistics; Essays in honor of Harold Hotelling. Stanford UP, 78-92.

Levene Test for Equality of Variances (18 July 2006). Retrieved 01 June 2009 from NIST/SEMATECH e-Handbook of Statistical Methods. US Commerce Department's Technology Administration Web site: http://www.itl.nist.gov/div898/handbook/eda/section3/eda35a.htm

Locke, E. A. (1975). Personal attitudes and motivation. Annual review of Psychology 26 457-80.

Lowry, R. T-test for significance of difference between means of two independent samples. (n.d.). Retrieved 2 June 2009 from Concepts and Applications of Inferential Statistics, Vassar University Web site: http://faculty.vassar.edu/lowry/webtext.html

Mahalanobis, P. C. (1936). On generalized distance in statistics. Proc. of Proceedings National Institute of Science, India.

Mantel, N. (1970). Why stepdown procedures in variable selection. Technometrics 12, 591-612.

March, J. \& Simon H. (1958). Organizations. New York: Wiley.

Mathieu, J. E. (1991). A cross-level nonrecursive model of the antecedents of organizational commitment and satisfaction. Journal of Applied Psychology 76, 607- 18 .

Mathieu J. E. \& D. M. Zajac. (1990). A review and meta-analysis of the antecedents, correlates, and consequences of organizational commitment. Psychological Bulletin 108, 171- 94. 
Measures of Skewness and Kurtosis. (n.d.). Retrieved 27 May 2009 from NIST/SEMATECH e-Handbook of Statistical Methods. US Commerce Department's Technology Administration, Web site: http://www.itl.nist.gov/div898/handbook/eda/section3/eda35b.htm

Miller, H. E., Katerberg, R. \& Hulin, C. L. (1979). Evaluation of the Mobley, Horner, Hollingsworth of employee turnover. Journal of Applied Psychology 64, 509-17.

Mobley, W. H. (1977). Intermediate linkages in the relationship between job satisfaction and employee turnover. Journal of Applied Psychology 62, 237-40.

Mobley, W. H., Griffith R. W., Hand, H. H. \& Meglino B. M. (1979). Review and conceptual analysis of the employee turnover process. Psychological Bulletin 86 (3), 493-522.

Mobley, W. H., Horner, S. O. \& Hollingsworth A. T. (1978). An evaluation of precursors of hospital employee turnover. Journal of Applied Psychology 63, 408-14.

Myers, Raymond H. 1990. Classical and modern regression with applications. New York: Duxbury.

Notes on Boxplots. (n.d.). Retrieved 05 June 2009 from School of Chemical and Mathematical Sciences - Murdoch University. Perth, Western Australia Web site: http://www.cms.murdoch.edu.au/areas/maths/statsnotes/samplestats/bo xplot.html\#Symmetry

Notes on Q-Q plots. (n.d.). Retrieved 05 June 2009 from School of Chemical and Mathematical Sciences - Murdoch University. Perth, Western Australia Web site: http://www.cms.murdoch.edu.au/areas/maths/statsnotes/samplestats/qq plot.html

Pearson, A. V. \& Hartley H. O. (1972). Biometrica tables for statisticians, volume 2. Cambridge, England: Cambridge UP.

Perreault, W. D., Behrman D. N. \& Armstrong G. M. (1977). Alternative approaches for interpretation of multiple discriminant analysis in marketing research. Journal of Business Research 7, 151-73. 
Porter, L. W. \& Steers R. M. (1973). Organizational, work, and personal factors in employee turnover and absenteeism. Psychological Bulletin $80,151-76$.

Porter, L. W., Steers, R. M., Mowday, R. T. \& Boulian, P. V. (1974). Organizational commitment, job satisfaction, and turnover among psychiatric technicians. Journal of Applied Psychology 59, 603-09.

Price, I. (2009). Determining if Skewness and Kurtosis are Significantly Nonnormal. Retrieved 27 February 2010 from University of New England, Armidale, NSW Web http://www.une.edu.au/WebStat/unit_materials/c4_descriptive_statistic s/determine_skew_kurt.html.

Riechheld, F. F. (2003, December). The one number you need to grow. Harvard Business Review, 46-54.

Royston, P. (1995). A remark on algorithm AS 181: the W-test for normality. Applied Statistics 44, 547-51.

Schaubroeck J, May, D. R. \& Brown F. W. (1994). Procedural justice explanations and reactions to economic hardship: a field experiment. Journal of Applied Psychology 79, 455- 60.

Schneider, D., Berent, M., Thomas, R. \& Krosnick, J. (2007). Measuring customer satisfaction and loyalty: Improving the 'Net Promoter' score. Proceedings of the Annual Conference of the World Association for Public Opinion Research, Berlin, Germany.

Shlens, J., (2010). Tutorial on Principal Component Analysis. Retrieved 26 February 2010 from Salk Institute for Biological Studies, and Institute for Nonlinear Science, University of California, San Diego Web site: fhttp://www.snl.salk.edu/ shlens/

Shapiro, S. S. \& Wilks M. B. (1965). An analysis of variance test for normality (complete samples). Biometrika 52, 591-611.

Sims, H. P., Szilagyi, A. D., \& Keller, R. T. (1976). The measurement of job characteristics. Academy of Management Journal 19 (2), 195-212.

SPSS FAQ: What does Cronbach's alpha mean? (n.d.). Retrieved 20 May 2009 from UCLA Academic Technology Services Web site: http://www.ats.ucla.edu/stat/SPSS/faq/alpha.html 
Steers, R. M. \& Mowday R. T. (1981). Employee turnover and post-decision accommodation processes. Research in organizational behavior, Vol. 3. Greenwich, CT: JAI.

Stepwise Multiple Regression (n.d). Retrieved 22 May 2009from Department of Psychology, East Carolina University Web site: http://core.ecu.edu/psyc/wuenschk/MV/MultReg/Stepwise.doc

Stepwise Regression $=$ Voodoo Regression (n.d.). Retrieved 22 May 2009 from Department of Psychology, East Carolina University Web site: http://core.ecu.edu/psyc/wuenschk/StatHelp/Stepwise-Voodoo.htm

Stone, C. J. (1985). Additive regression and other nonparametric models. Annals of Statistics 13, 689-705.

Streiner, D. L. \& Norman G. R. (1989). Health measurement scales; A practical guide to their development and use. New York: Oxford UP.

Sweeney, P. D. \& McFarlin, D. B. (1993). Workers' evaluations of the 'ends' and 'means': an examination of four models of distributive and procedural justice. Organizational Behavior and Human Decision Processes 55, 23-40.

Teknomo, K. (2009). Mahalanobis Distance. Retrieved 25 August 2009 from People.revoledu.com Web site: http://people.revoledu.com/kardi/tutorial/Similarity/MahalanobisDistan ce.html

Tibshirani, R. (1996). Regression shrinkage and selection via the lasso. Journal of the Royal Statistical Society B 58, 267-88.

Turrban, D. B. (2003). Firm reputation and applicant pool characteristics. Journal of Organizational Behavior 24, 733-51.

Vroom, V. (1964). Work and motivation. New York: John Wiley and Sons.

Wilkinson, L., Blank G. \& Gruber, C. (1996). Desktop data analysis with SYSTAT. Upper Saddle River, NJ: Prentice Hall.

Williams, F. \& Monge P. (2001). Reasoning with Statistics. 5th ed. Orlando, FL: Harcourt

Would You Recommend Us? (2006, January 30), Retrieved 23 February 2010 from Business Week wite: http://www.businessweek.com/magazine/content/06_05/b3969090.htm 
Yee, T. (January 15, 2010). The VGAM Package for R. Retrieved 06 Dec. 2009 from Department of Statistics, The University of Auckland, New Zealand Web site: http://www.stat.auckland.ac.nz/ yee/VGAM/ 


\section{Classification and Analysis of College Student Leadership Data}

\section{Introduction}

To briefly introduce this section, this work is comprised of a review of the leadership literature, particularly as it relates to personal development, followed by the validation of an assessment tool, and results from a survey dataset. This application focuses specifically on the development of college students as emerging leaders. Leadership studies colleagues Drs. Marcy Shankman, and Scott Allen sought this author's assistance in the development and validation of an assessment tool that would measure a student's selfperception of their own leadership behaviors. The collaborative work also resulted in a deeper focus on gender differences and involvement through the lens of emotionally intelligent leadership (Shankman, Haber, Facca, Allen, 2010).

Additional analyses were conducted to further demonstrate the appropriate applicability of statistical techniques given the dataset. These included an attempt at discriminant analysis which was not particularly fruitful given a lack of solid outcome variables since no external measurement of leadership effectiveness was available. However, cluster analysis proved to be useful in discerning groups and related leadership behaviors. Factor analysis revealed underlying constructs, and reliability analysis was used to validate the assessment tool.

The primary contribution is the development of a tool to help students selfassess their current leadership behaviors and focus on behaviors that can facilitate personal development and improvement of leadership skills. The assessment tool, or Emotionally Intelligent Leadership Inventory, is in press with Jossey-Bass, a Wiley Imprint, and will be available August 2010, as part of a collection of resources by Shankman, Allen, and Facca which also include a student workbook, a facilitator's guide, and a development guide. 


\subsection{College Student Leadership - Issues, Constraints and Literature}

In his book, Deeper Learning in Leadership: Helping College Students Find the Potential Within, Roberts (2007) suggests that "leadership learning experiences will have the greatest substance and impact only when they take into account the existing research and the many available theories on leadership and its dynamics" (p. 41). Too often, leadership development lacks a foundation in theory which can limit the experience in a number of ways. Many (in varied industries) have explored the challenges inherent in translating theory into practice (e.g., Higginson, 2004; Rafferty, Allcock \& Lathem, 1996). One challenge in the theory to practice gap can be the theory's ability to walk the tightrope of capturing the complexity of a topic while making the information accessible and digestible for the masses.

In the 1970s college student personnel administrators began writing about and working in the area of leadership development. One of the first comprehensive examinations of the topic was compiled by Roberts (1981), and while the topic remained present in subsequent years, it seems to have gained momentum in recent years. While there is a considerable amount of activity (Komives, Dugan, Owen, Slack \& Wagner, 2006) and writing (e.g., Marshall \& Hornak, 2008; Shankman \& Allen, 2008; Komives \& Wagner, 2009; Kouzes \& Posner, 2008) on the topic, few scholars have developed theories or models specific to a collegiate audience.

However, two major models have been specifically designed with students in mind. The first, The Social Change Model of Leadership Development was funded by the Higher Education Research Institute (HERI). The authors had two primary goals for the model: (1) To enhance student learning and development; more specifically, to develop in each student participant greater leadership skills; and (2) to facilitate positive social change at the institution or in the community; to undertake actions which will help the institution/community to function more effectively and humanely (HERI, 1996, p. 19). The model examines leadership development from three different levels: (1) The Individual; (2) The Group; and (3) The Community/Society. In addition, the authors developed the "7 $\mathrm{Cs}$ " for leadership development for social change.

The second, is the Leader Identity Development Model (LID). The LID Model, identified six distinct stages: Awareness (Stage One); Exploration/Engagement (Stage Two); Leader Identified (Stage Three); Leadership Differentiated (Stage Four); Generativity (Stage Five); Integration/Synthesis (Stage Six). In essence, students move from a more hierarchical (the leader has the position) view of leadership to more of 
connected/relational model of leadership (Roberts, 2007). According to Komives et al. (2009), "As that engagement happened, the student changed their view of self with others beginning in a dependent mode when in a follower role, then feeling independent when in a leader role, and finally recognizing interdependence with others" (p. 15).

This study introduces a third model designed specifically for young adults and emerging leaders. The goal was to provide a method to statistically assess an individual's leadership skill set, categorize them in comparison to others, and ultimately, based on the dataset, make suggestions for development. First, two primary theories are examined that comprise Emotionally Intelligent Leadership (EIL) - Emotional Intelligence and Contingency Theory. Second, an overview of the method is provided, the sample discussed, and then an examination of the research results based on the Emotionally Intelligent Leadership Inventory (EILI).

The Roots of EIL

\section{Contingency Theory}

Throughout the late 1960s and 1970s, the Contingency Model of leadership was one of the most widely studied theories of leadership (e.g., Strube \& Garcia, 1981). By the mid-1990s, Contingency Model had been supported by two published meta-analyses, one of which was based on more than 145 tests of the hypotheses, making the Contingency Model the most validated leadership theory (Fiedler, 1995). However, today, other than a feature in some textbooks, Contingency Model does not seem to "live" in the community of leadership scholars and practitioners. The theory never took root, even though it dominated leadership literature for more than a decade. Research has waned and practitioners do not seem to be interested. While Blake and Mouton's (1978) Managerial/Leadership Grid Theory examines the leader's focus on "relationship" and "task," Contingency Theory focuses on the importance of a leader's match to a given situation. According to Fiedler and Mahar (1979), "a leader's performance is based on two interacting factors: (a) the individual's goals and needs or motivational structure and (b) the degree to which the situation provides the leader with control and influence over the outcome of the task" (p. 248). In essence, the theory is focused on the leader changing the situation, rather than changing his or her "style."

One challenge of Contingency Theory is that it is nearly impossible to categorize every possible situation a leader may face. Further, it does not take into account the notion that leaders have the ability to grow and adapt to given situations. Finally, it would be difficult to appoint and remove individuals 
from positions of authority simply because their skills do not match a given situation. Regardless, Fiedler brought an important concept to the table when he suggested that leadership is relationship between the leader, the followers, and the context. Scholar David Day suggests, "These kinds of training approaches, however, ignore almost 50 years of research showing leadership to be a complex interaction between the designated leader and the social and organizational environment" (Day, 2001). Leadership is complicated and success or failure is a mix of what might be considered an almost infinite number of behavioral variables that make up the formal/informal leader, the followers (social environment) as well as variables related to the context (organizational environment).

\section{Emotional Intelligence}

So what attributes (competencies, skills) does one need to embody to successfully navigate such an environment? How does one have to behave to best approach the challenges associated with effective leadership (let alone the measurement of such)? Emotional intelligence (EI) offers a framework for approaching the complexities facing leaders today. Not only does EI promote a way of being that will serve a leader well (a focus on self awareness, self regulation, and relationship management), it highlights a number of competencies that support leadership from a behavioral perspective, (optimism, influence, conflict management, etc.).

Although a relatively new approach to thinking about intelligence, performance or personality, a number of interesting findings have been associated with Emotional Intelligence. For instance, in their meta-analytic study, Schutte, Malouff, Thorsteinsson, Bhullar \& Rooke (2007) found that emotional intelligence is associated with better health. Moreover, studies associated with Bar-On's EQ-I, have found that there is a moderate yet significant relationship between EI and physical and psychological health and that the "Bar-On model is capable of identifying and predicting who will perform well at school and who will not" (Bar-On, 2006, p.14). The same is true for predicting who will perform well at work (Bar-On, 2006, p. 15). In health care settings, Akerjordet \& Severinsson (2008) report that emotional intelligence has been correlated to increased job performance, resilience to stressful situations and better capacity to demonstrate a caring attitude. In addition, emotionally intelligent health care providers "nurture a greater sense of safety and provide better management performance" (Akerjordet \& Severinsson, 2008, p. 569). In addition, in a study of 300 senior executives, Spencer (1997) found that the competencies of influence, team leadership, organizational awareness, self-confidence, achievement drive, and leadership 
were identified in the high performers vs. average employees. Finally, along with management performance, a number of studies show that EI leads to effective leadership (e.g., Kerr, Garvin, Heaton, \& Boyle, 2006; Goleman, Boyatzis \& McKee, 2002; Bar-On, 2006; Cherniss, 1999).

The term emotional intelligence (EI) was coined by Peter Salovey and John Mayer in 1990, based on the work of many before them (e.g., Gardner 1983; McClelland, 1973; Thorndike \& Stein, 1937). Many of these scholars assumed that cognitive intelligence (IQ) alone does not lead to success (or effectiveness). In fact, Hunter and Hunter (1984), concluded that intelligence accounts for around $25 \%$ of the variance in high performing employees. Others have experienced similar results (e.g., Sternberg, 1996, Singh, 2006). Salovey \& Mayer's (1990) original definition of EI was, "a form of social intelligence that involves the ability to monitor one's own and others' feelings and emotions, to discriminate among them, and to use this information to guide one's thinking and action" (p. 189). Today, there are three primary approaches to the construct of Emotional Intelligence which can make it a confusing concept to understand and grasp (Caruso, 2003). However, it is important to understand that all, at their core, believe that EI is about the "abilities to recognize and regulate emotions in ourselves and others" (Goleman, 2000, p. 2). Goleman (2000) explains the three primary approaches to EI and suggests that Salovey \& Mayer "framed EI within a model of intelligence" (p. 2), while Bar-On framed his model of EI in personality theory. Goleman (2000) suggests his model as a theory of performance.

As mentioned, the "competing" EIL approaches may not seem all that different at first blush. However, Bar-On (2006) effectively distinguishes the three approaches:

(a) The Salovey-Mayer model (Mayer \& Salovey, 1997) which defines this construct as the ability to perceive, understand, manage and use emotions to facilitate thinking, measured by an ability based measure (Mayer et al., 2002); (b) the Goleman model (1998) which views this construct as a wide array of competencies and skills that drive managerial performance, measured by a multi-rater assessment (Boyatzis et al., 2001); (c) The Bar-On model (1997b, 2000) which describes a cross section of interrelated emotional and social competencies, skills and facilitators that impact intelligent behavior, measured by self report $(1997 a, 1997 b)$ within a potentially expandable multi-modal approach including interview and multi-rater assessment (Bar-On \& Handley, 2003a, 2003b). (p. 2).

In their book Primal Leadership, Goleman, Boyatzis and McKee (2002) suggest that: 
Primal leadership operates at its best through emotionally intelligent leaders who create resonance. Underlying that proposition is a theory of performance, one that surfaces the links between the neurology of the four fundamentals of emotional intelligence and the EI competencies that build on those fundamentals. These EI competencies are in turn the building blocks of the modes of leadership that prime resonance in a group (p. 38).

A competency is "an underlying characteristic of a person which results in effective and/or superior performance in a job" (Boyatzis, 1982, p. 21). These competencies are housed under the fundamentals of self-awareness, self management, social awareness and relationship management. Goleman et al. (2002) define self-awareness in the following way: "Simply put selfawareness means having a deep understanding of one's emotions, as well as one's strengths and limitations and one's values and motives" (p. 40). Selfmanagement addresses an individual's ability to regulate his or her impulses and behaviors. This is extremely important because of the contagious nature of emotions. Social awareness captures a leader's ability to express his or her feelings with those around them. The leader's ability to empathize with those around them will likely determine their ability to remain in tune with those in their sphere or the organizations as a whole. Finally, relationship management focuses on a leader's ability to manage the emotions of those around them. To do so, the leader must have a keen sense of his or her own emotional state.

\section{The Emotionally Intelligent Leadership Model}

Emotionally Intelligent Leadership, at the heart, is a piece of integrative scholarship. Ernest Boyer (1990) of the Carnegie Foundation suggested that, "...the work of a scholar also means stepping back from one's investigation, looking for connections, building bridges between theory and practice, and communicating one's knowledge effectively to students" (p. 16). More specifically, he suggested "By integration, we mean making connections across the disciplines, placing the specialties in a larger context, illuminating data in a revealing way...serious, disciplined work that seeks to interpret, draw together, and bring new insight to bear on original research" (p. 18-19). Rather than looking at the many theories of leadership as isolated and competing concepts, here they are viewed through an inclusive lens leading to synthesis. Doing so not only aligns a number of similar concepts from the various theories, but also provides a more holistic perspective on the complex dynamic of leadership.

Emotionally intelligent leadership finds its roots in leadership theory, contingency theory and emotional intelligence. First, a number of leadership 
scholars have informed this thinking and are included in the development of the model - for instance, Heifetz \& Linsky's (2002) work around the context in which a leader does his or her work is captured. In addition, the need to inspire others is promoted as is highlighted in Kouzes and Posner's Five Practices (Kouzes \& Posner, 1995), Transformational Leadership (Bass, 1985), charismatic leadership (Conger, 1989), relational leadership (Komives, Lucas \& McMahon, 2006) and so forth. Likewise, the model includes concepts such as optimism and self-efficacy/self-confidence which are heralded by Avolio \& Luthans (2006), and Goleman, Boyatzis \& McKee (2002) as crucial ingredients for effective leadership to occur. Throughout the work authors are identified and categorized who promote or put forth similar viewpoints. For instance, Avolio (2005), Goleman, Boyatzis and McKee (2002), McCauley and Van Velsor (2005), and HERI (1996) all suggest that self-awareness is an important component of effective leadership. In essence, rather than promote one approach or theory, this array (transformational leadership, emotional intelligence, contingency theory, social change model, leader-member exchange, center for creative leadership, etc.) is incorporated under one roof.

The Emotionally Intelligent Leadership (EIL) model aligns most closely with Goleman's approach to Emotional Intelligence. The focus on capacities and helping college students perform more effectively when serving in a leadership role coincides with Goleman's competency model. However, EIL is comprised of three facets (consciousness of context, consciousness of self, and consciousness of others), which were also inspired by the work of HERI (1996), Fiedler (1979), Kellerman (2004) and Heifetz (1994). These approaches add a dimension not present in other models of EI - the dimension of context as a fundamental component. For instance, in her book $\mathrm{Bad}$ Leadership, Kellerman (2004) analyses each case by examining first the context, then the followers and finally the leader. The result is a rich story of how these three elements converged to create the result it did (in this case, bad). Thus, an emotionally intelligent leader must respond to and regulate the emotions in self, others, and the larger context at hand. Heifetz (1994) suggests that 'Leadership is a razor's edge because one has to oversee a sustained period of social disequilibrium during which people confront the contradictions in their lives and communities and adjust their values behavior to accommodate new realities" (p. 127). To respond to this sense of disequilibrium, Heifetz suggests that the leader is responsible for "holding" followers through this period of discomfort. This is also known as a holding environment - "A holding environment is a space formed by a network of relationships within which people can tackle tough, sometimes divisive questions without flying apart...In a holding environment, with structural, 
procedural, or virtual boundaries, people feel safe enough to address problems that are difficult, not only because they strain ingenuity, but also, they strain relationships" (Heifetz \& Linsky, 2002, p. 102-103).

\section{Three Facets and Twenty-one Capacities of Emotionally Intelligent Leadership}

As mentioned, EIL focuses on three facets (consciousness of context, consciousness of self, and consciousness of others). Consciousness of context focuses on the environment in which leaders and followers work. Consciousness of self is being aware of oneself in terms of abilities and emotions. Consciousness of others is attending to, and working in, relationship with others and the role they play in the leadership equation. The three facets of EIL relate to a person's awareness, which is necessary but not sufficient for demonstrating emotionally intelligent leadership.

To bring this awareness to the surface and show action, the model is comprised of 21 capacities. The word capacity is used because many of these behaviors, skills and ways of being can be learned. According to the American Heritage Dictionary, capacity is defined as "ability to perform or produce; capability." This word is chosen because the assumption, like that of Arvey (2006; 2007), is that everyone has the capacity to develop the ability to lead others effectively. An in-depth review of the theoretical roots of each capacity is beyond the scope of this work. However, it is important that a cursory definition is provided with an explanation for each. As previously mentioned, these capacities are integrative in nature. They integrate the thinking of several scholars and theorists into one model of effective leadership (e.g., Avolio \& Gardner, 2005; Bass 1995; Goleman, Boyatzis \& McKee, 2002; Heifetz, 1994; Fiedler, 1972; Blanchard, Zigarmi, \& Nelson, 1993; Kouzes \& Posner, 2007; HERI, 1996).

Given that the context is the environment in which leaders and followers work (e.g., Fiedler, 1996; Heifetz, 1994; HERI, 1996), EIL recognizes that the settings and situations that leaders experience are always different, and may even be constantly changing. For example, people in the organizational context may be open to being led, or not. Shankman \& Allen (2008) suggest that each new context requires a different set of knowledge, skills, and abilities on the part of leaders and followers. A leader's ability to accurately diagnose these dynamics will lead to success or failure (e.g., Day and Lance, 2004; London, 1999; Goleman, et al., 2002; Hartley \& Hinksman, 2003; Heifetz and Linsky, 2002; and Zenger, Ulrich, \& Smallwood, 2000). Once diagnosed, the 
road to success or failure is dependent in large measure on the knowledge, skills, and abilities of those involved. The two capacities that comprise this consciousness of context facet include:

- Group Savvy - Interpreting the situation and/or networks of an organization

- Environmental Awareness - Intentionally thinking about the environment in any leadership situation especially the physical, psychological, and organizational elements. Harvard faculty Marty Linsky and Ronald Heifetz (2002) call this "getting on the balcony" (p. 8). This means an individual has the ability to observe, in real time, the group dynamics and factors present in the environment..

A number of authors have addressed the importance of self-awareness as a fundamental construct of effective leadership (e.g., Avolio, 2005; Conger, 1993; Goleman, Boyatzis and McKee, 2002; HERI, 1996; London, 2002; Van Velsor \& McCauley, 2005). Consciousness of self requires knowing oneself and one's values, as well as how one's actions affect others (HERI, 1996). Since this is a lifelong endeavor, Shankman \& Allen (2008) suggest that it's not important that introspection result in concrete answers, but it is the process that is important. This research aims to help understand that process. In addition, it must be noted that consciousness of self requires actions that are aligned with one's words. This fosters trust, which is required of leadership (Shankman \& Allen, 2003, p. 26). The nine capacities that comprise this SELF facet include:

- Emotional self-perception - Identifying one's emotions and reactions and their impact on oneself. This capacity enables one to differentiate between emotions felt and actions taken.

- Honest self-understanding - Being aware of one's own strengths and limitations

- Healthy self-esteem - Having a balanced sense of self; holding oneself in check, evidencing humility

- Emotional self-control - Consciously moderating one's own emotions and reactions and regulating them

- Authenticity - Being transparent and trustworthy so that words match actions. This involves commitment and follow-through; being true to one's feelings and ideas; effectively aligning actions with values

- Flexibility - Being open and adaptive to changing situations 
- Achievement - Being driven to improve according to personal standards

- Optimism - Being positive and displaying a positive regard for the future.. Research suggests that being optimistic is an important element of emotional intelligence and leadership (Avolio \& Luthans, 2006; Goleman, Boyatzis, \& McKee, 2002).

- Initiative - Wanting and assertively seeking opportunities

Consciousness of others requires one to empathize, inspire, influence, coach, manage conflict and effect change. The challenge is in the fact that context is dynamic with infinite number of variables affecting leadership, thus requiring the leader to understand others' communication patterns and preferences, desires, expectations, and work style to name just a few of the affecting variables. In perhaps the first article to truly focus on others (followers) In Praise of Followers Kelly (1988) suggested the following:

Leaders matter greatly. But in searching so zealously for better leaders we tend to lose sight of the people these leaders will lead. Without his armies, after all, Napoleon was just a man with grandiose ambitions. Organizations stand or fall partly on the basis of how well their leaders lead, but partly also on the basis of how well their followers follow. (p. 142)

In other words, followers are an essential area of focus for effective leadership. It is leaders and followers working together that yields results (or lack thereof). In her book Bad Leadership, Barbara Kellerman underscores this notion and places at least part of the blame for bad leadership on followers. Kellerman (2004) suggests that "we can't expect to reduce the number of bad leaders unless we reduce the number of bad followers" (p. 21). To this end, Chaleff (2003) suggests that no leader over a long period of time can sustain their success unless they have a group of supporters willing to stand up and share their perspective. All of us are leaders and followers and as Kellerman suggests (2008), "we are all, every one of us, followers first" (p. xxii). To help leaders (and followers) maintain this connection, there needs to be an acute consciousness of others. The ten capacities that comprise this facet include:

- Empathy - Understanding others from their perspective; requires a high level of self-awareness as well as awareness of others.

- Citizenship - Recognizing and fulfilling one's responsibility for others or for the group. "To be a good citizen is to work for positive change 
on behalf of others and the community" (Higher Education Research Institute, 1996, p. 23); requires giving for the benefit of others.

- Inspiration - Motivating and moving others toward a shared vision Leaders generate feelings of optimism and a commitment to organizational goals and vision by communicating ideals and vision for the organization through verbal cues, behaviors and internal and external relationships (Bass, 1985).

- Influence - Demonstrating skills of persuasion - Bass (1997) suggests that "leaders display conviction; emphasize trust; take stands on different issues; present their most important values; and emphasize the importance of purpose, commitment and ethical consequences of decision making. Such leaders are admired as role models in generating pride, loyalty, confidence, and alignment around a shared purpose" (p. 133).

- Coaching - Helping others enhance their skills and abilities — doing what is necessary to train and prepare others for the tasks or challenges at hand.

- Change agent - Seeking out and working with others toward new directions. Changes may benefit one person, an organization, or a whole community. Change agents possess creative problem solving skills, and are comfortable with risk taking.

- Conflict management - Identifying and resolving problems and issues with others

- Developing relationships - Creating connections between, among, and with people - In relation to leadership, authorities have discussed the centrality and importance of relationships for years (Bass \& Avolio, 1994; Blake \& Mouton, 1978; Fiedler \& Chemers, 1984; Graen \& UhlBien, 1995).

- Teamwork - Working effectively with others in a group - knowing how to build a team and a role model of collaboration.

- Capitalizing on differences - Building on assets that come from differences with others; requires flexibility in terms of a desire to learn, being open to possibilities not considered, and even changing one's mind. 
Chris Lowney (2003, p.110) discusses Jesuit self-awareness and its similarities to Goleman's notion of emotional intelligence. Both wanted to identify personal traits essential to successful leadership. Goleman (1998, p. 94) summarizes five core competencies of emotional intelligence which include:

- Self-awareness - recognizing one's own moods, emotions and drives

- Self-regulation - controlling or redirecting disruptive impulses and moods; and the propensity to suspend judgment and think before acting

- Motivation - passion to work for reasons beyond money and status

- Empathy - ability to understand the emotional makeup of others

- Social skill - proficiency in managing relationships and building networks; finding common ground and building rapport

Lowney suggests the 450-year-old company of the Jesuits took it one step further by training their recruits "how" to impart these skills. Through a consistent focus on knowing oneself (and the practice of Loyola's Spiritual Exercises), recruits emerged with invaluable personal strengths including:

- The ability to systematically recognize one's own weaknesses and negative habits

- An integrated worldview, vision and value system

- Appreciation of oneself as loved and important

- Ability to meditate and reflect daily

- A method for discerning choices and making decisions

Much work has been done to study leaders, the personality characteristics of leaders, and how leaders perceive themselves and others. This work aims at capturing the importance of one's consciousness of context, and understanding how this facet of consciousness is self-perceived by college students. All three facets, consciousness of context, self and others are measured and evaluated and the results discussed.

\section{Involvement, Gender, Leadership \& Emotional Intelligence}

Research demonstrates that involvement in student groups and organizations is a significant predictor for college students' leadership development (Astin, 1993; Hernandez, Hogan, Hathaway, \& Lovell, 1999; Cooper, Healy, \& Simpson, 1994). While involvement in organizations is found most often to positively contribute to students' leadership outcomes, involvement in too many different types of organizations can also negatively contribute to students' leadership development (Dugan \& Komives, 2007). One study found breadth of involvement was negatively associated with outcome scores 
of commitment for college men (Haber \& Komives, 2009). Additional findings related to gender emerged around involvement; women tended to be involved in student organizations more often than men, and involvement in student organizations was a significant predictor of leadership outcome more often for women than men (Haber, 2006).

Gender differences also exist in how women and men engage in leadership. Literature and research on leadership styles and capacities by gender suggest that women adopt more relational, democratic, and transformational approaches to leadership than men (Eagly \& Carli, 2007; Northouse, 2004; Eagly, Johannesen-Schmidt, \& van Engen, 2003; Dugan, Komives \& Segar, 2008; Haber \& Komives, 2009). Gender differences in leadership exist in the arenas of leadership traits, leadership styles, and overall approaches and perspectives of leadership. Men and women differ in personality traits that are predictors of leadership (Eagly \& Carli; Judge, Bono, Ilies, \& Gerhardt, 2002). Women's personality traits tend to reflect agreeableness, warmth, positive emotions, extraversion, and openness to feelings, while men's personality traits tend to reflect greater assertiveness (Costa, Terracciano, \& McCrae, 2001; Eagly \& Carli). These findings on traits are reflective of similar findings on leadership behavior.

Research on gender differences in leadership style, which is a behavioral measure of leadership, indicates that women practice more democratic leadership while men tend to demonstrate more autocratic leadership (Eagly \& Carli, 2007). Democratic leadership is characterized by a participative and collaborative style, which emphasizes shared power and decision-making, while an autocratic style is more hierarchical and directive (Northouse, 2004). In a study examining the leadership styles and experiences of college student women holding formal leadership roles, the participants used such terms as "nonhierarchical, interactive, accessible, one-to-one, equality and teammember" (Romano, 1996, p. 679) when describing their approaches to leadership. Another study emphasized women's leadership styles as being dependent on the larger environment and the particular role in which they are operating. For example, in male-dominated organizations or industries, women may demonstrate more autocratic leadership, which tends to reflect that of their male colleagues (Eagly \& Carli).

Women also demonstrate higher levels of transformational leadership than men (Eagly \& Carli, 2007; Eagly et al., 2003). Transformational leadership, characterized by inspiring and empowering followers, developing followers and responding to their needs, and aligning follower, leader, and organizational goals, is associated with greater follower satisfaction, 
performance, and commitment. Additionally, transformational leadership is associated with greater overall leader and organizational performance (Bass \& Riggio, 2006).

The recent Multi-Institutional Study of Leadership (MSL) examined college students' socially responsible leadership development, which is informed by the social change model of leadership (HERI, 1996). Socially responsible leadership emphasizes a collaborative, accessible, values-based, and serviceoriented process of leadership that includes three levels of the model and eight outcomes. The individual level focuses on consciousness of self, congruence, and commitment; the group level includes collaboration, common purpose, controversy with civility; the societal level includes citizenship. The overall goal of the model is change (Komives \& Wagner, 2009).

Findings from MSL research indicate that women report significantly higher levels of socially responsible leadership. A single-campus study identified that women demonstrated significantly higher levels than men in all three of the group outcomes and the individual outcomes of congruence and commitment (Haber, 2006; Haber \& Komives, 2009). The national study identified more prominent gender differences; women reported significantly higher scores than men in all of the outcomes with the exception of change, in which there were no significant differences by gender (Dugan et al., 2008). Interestingly, results from this study also identify that men demonstrated significantly higher measures of leadership self-efficacy, or confidence in their ability to lead, than women (Dugan \& Komives, 2007). Examining these findings side by side, women demonstrated higher competence in leadership, while men demonstrated higher confidence. Higher levels of leadership selfefficacy among men were also evident in Kezar \& Moriarty's (2000) study on college student leadership.

Women's more collaborative and participative style is also reflected in research on emotional intelligence. The tendency for women to demonstrate higher degrees of emotional intelligence than men has been measured in various ways, including how people perceive, use, understand, and manage emotions (Brackett, Rivers, \& Shiffman, 2006). Research also suggests differences between the genders in variables of emotional intelligence such as adaptability, stress management, intrapersonal skills (i.e. awareness and connection to inner-self), interpersonal skills, and general mood (Mandell \& Pherwani, 2003). Recent research, however, suggests conflicting findings for younger generations. While significant differences in women and men's emotional intelligence existed for older generations (mean age of late forties), 
with women scoring higher than men, no gender differences existed for the younger, college-aged generation (Guastello \& Guastello, 2003).

The literature suggests that women tend to value others in their approach to leadership as well as demonstrate intrapersonal strengths more so than men. More specifically, women demonstrate a preference for the interpersonal dynamics of leadership, particularly working with others and groups in a collaborative way. Women also tend to demonstrate higher levels of intrapersonal skills than men. While there is evidence of women's greater intrapersonal and interpersonal skills, men's self-reported self-efficacy in their leadership abilities exceeds that of women. Much research on emotional intelligence and leadership suggests significant differences by gender, with women demonstrating higher levels than men, yet a recent study examining college students does not show significant gender differences. Thus, examining gender differences in leadership and emotional intelligence among college students warrants additional research.

The examination of leadership and emotional intelligence through a model that integrates the two may shed additional light on the differences or similarities in college-aged women and men's emotionally intelligent leadership. Additional information on gender differences in leadership and emotional intelligence can allow for a better understanding of students' leadership development and awareness of themselves, others, and the larger environment. Investigating the interplay between involvement and gender will further these understandings, and can also help college student educators by helping them develop well-rounded and emotionally intelligent leaders.

This work enables a precise focus on measuring a college student's selfperceived emotional intelligence skills in leadership settings. It considers consciousness of context, self and others as underlying constructs, investigates the variables associated with differences between varied levels of consciousness in leadership settings, and investigates gender differences and involvement through the lens of emotionally intelligent leadership. 


\subsection{Description of the Dataset}

A total of 566 students from 139 colleges and universities in the United States completed an online assessment of their leadership skills in the spring of 2009. Assessment authors Shankman and Allen composed the research tool as an eventual supplement to their book, Emotionally Intelligent Leadership; $A$ Guide for College Students (2008). In their model of emotionally intelligent leadership (EIL), three fundamental facets contribute to the leadership dynamic: consciousness of context, self, and others. Student were asked to use a five-point scale to indicate the extent to which they intentionally participated in or focused on a total of twenty-four variables when serving in a formal or informal leadership role. The scale was assigned as follows:

\begin{tabular}{|ccccc|}
\hline Never & Infrequently & Sometimes & Frequently & Always \\
\hline 1 & 2 & 3 & 4 & 5 \\
\hline
\end{tabular}

Students provided a rating for each variable to the extent that it completed the sentence at the top of Table 1.2.1

\begin{tabular}{|l|} 
When serving in a formal or informal leadership role, I... \\
\hline Take time to understand the informal traditions of the group \\
\hline Learn the expressed and implicit values of the group \\
\hline Monitor how my emotions affect my interactions with others \\
\hline Work on my limitations \\
\hline Tailor my leadership style to the situation \\
\hline Capitalize on my strengths \\
\hline Understand how the group's environment influences my leadership style \\
\hline Help others enhance their skills and abilities \\
\hline Understand how the group members relate to one another \\
\hline Follow through on my commitments \\
\hline Work to build a sense of team \\
\hline Think about how my leadership style aligns with group culture \\
\hline Establish a positive tone \\
\hline Think about how my decisions are received by group members \\
\hline Improve my abilities \\
\hline Identify external influences on the group \\
\hline Consider the needs of others in the group \\
\hline Recognize the patterns of behavior in the group \\
\hline Reflect on how my actions align with my values \\
\hline Understand the priorities of others in the group \\
\hline Listen carefully to what is and isn't being said by group members \\
\hline Work with others toward a shared goal \\
\hline Work to resolve conflicts within the group \\
\hline Align different viewpoints within the group \\
\hline Table 1.2.1 Leadership behavior questions \\
\hline
\end{tabular}

The 24 questions were designed to capture three constructs; consciousness of context, consciousness of self, and consciousness of others within a leadership 
setting. Students simply added their scores for the variables that fell into each construct category, arriving at a self-score between 8 and 40 on each of the three constructs (assuming they responded at least "1" to each of the questions). Later we will discuss the development and testing of the reliability of the constructs using factor analysis, but at this juncture the descriptive statistics for each variable will be discussed by construct groupings (consciousness of context, self and others).

\section{Consciousness of Context}

To simplify scoring, the three constructs are each comprised of eight variables. Table 1.2.2 provides the total number of respondents for each variable that loads on the consciousness of context factor, its range, mean and standard deviation.

Recognition of patterns of behavior in the group scored high at 3.99, followed by one's tailoring of their leadership style to the situation (3.98), and learning the expressed and implicit values of the group (3.95). Later, some quite interesting and significant differences between genders as well as high-scorers, and those who are more actively involved in student organizations, will be discussed.

\begin{tabular}{|c|c|c|c|c|c|}
\hline \multicolumn{6}{|l|}{$\begin{array}{l}\text { Consciousness of Context } \\
\text { Descriptive Statistics }\end{array}$} \\
\hline & $\mathrm{N}$ & Min & Max & Mean & $\begin{array}{l}\text { Std. } \\
\text { Dev. }\end{array}$ \\
\hline $\begin{array}{l}\text { Recognize the patterns of behavior in the } \\
\text { group }\end{array}$ & 566 & 1 & 5 & 3.99 & .776 \\
\hline Tailor my leadership style to the situation & 564 & 1 & 5 & 3.98 & .850 \\
\hline $\begin{array}{l}\text { Learn the expressed and implicit values of } \\
\text { the group }\end{array}$ & 566 & 2 & 5 & 3.95 & .790 \\
\hline $\begin{array}{l}\text { Understand how the group members relate } \\
\text { to one another }\end{array}$ & 565 & 1 & 5 & 3.93 & .845 \\
\hline $\begin{array}{l}\text { Take time to understand the informal } \\
\text { traditions of the group }\end{array}$ & 566 & 1 & 5 & 3.84 & .826 \\
\hline $\begin{array}{l}\text { Understand how the group's environment } \\
\text { influences my leadership style }\end{array}$ & 565 & 1 & 5 & 3.74 & .908 \\
\hline Identify external influences on the group & 564 & 1 & 5 & 3.72 & .867 \\
\hline $\begin{array}{l}\text { Think about how my leadership style aligns } \\
\text { with group culture }\end{array}$ & 566 & 1 & 5 & 3.68 & .927 \\
\hline
\end{tabular}




\section{Consciousness of Self}

College students tended to perceive themselves as quite strong on following through on commitments (4.46), working with others toward a shared goal (4.36), and establishing a positive tone (4.30).

\begin{tabular}{|c|c|c|c|c|c|}
\hline \multicolumn{6}{|l|}{$\begin{array}{l}\text { Consciousness of Self } \\
\text { Descriptive Statistics }\end{array}$} \\
\hline & $\mathrm{N}$ & Min & $\operatorname{Max}$ & Mean & Std. Dev. \\
\hline Follow through on my commitments & 563 & 2 & 5 & 4.46 & .651 \\
\hline Work with others toward a shared goal & 562 & 2 & 5 & 4.36 & 690 \\
\hline Establish a positive tone & 562 & 1 & 5 & 4.30 & .725 \\
\hline Capitalize on my strengths & 564 & 2 & 5 & 4.13 & .725 \\
\hline $\begin{array}{l}\text { Reflect on how my actions align with } \\
\text { my values }\end{array}$ & 564 & 2 & 5 & 4.08 & .817 \\
\hline Improve my abilities & 561 & 1 & 5 & 4.06 & .756 \\
\hline $\begin{array}{l}\text { Monitor how my emotions affect my } \\
\text { interactions with others }\end{array}$ & 566 & 1 & 5 & 3.93 & .870 \\
\hline Work on my limitations & 561 & 1 & 5 & 3.53 & .884 \\
\hline
\end{tabular}

Table 1.2.3 Consciousness of Self -Descriptive Statistics

\section{Consciousness of Others}

Considering their leadership roles with regard to others, students rated their consideration of the needs of others in the group quite high at 4.25 , followed by working with others to resolve conflicts within the group at 4.16 , and working to build a sense of team at 4.12 .

\section{Consciousness of Others Descriptive Statistics}

\begin{tabular}{|l|l|l|l|l|l|}
\hline & $\mathrm{N}$ & Min & Max & Mean & Std. Dev. \\
\hline $\begin{array}{l}\text { Consider the needs of others in the } \\
\text { group }\end{array}$ & 566 & 2 & 5 & 4.25 & .728 \\
\hline $\begin{array}{l}\text { Work to resolve conflicts within the } \\
\text { group }\end{array}$ & 565 & 1 & 5 & 4.16 & .832 \\
\hline Work to build a sense of team & 560 & 1 & 5 & 4.12 & .798 \\
\hline $\begin{array}{l}\text { Listen carefully to what is and isn't } \\
\text { being said by group members }\end{array}$ & 565 & 1 & 5 & 4.10 & .845 \\
\hline $\begin{array}{l}\text { Think about how my decisions are } \\
\text { received by group members }\end{array}$ & 564 & 2 & 5 & 4.07 & .834 \\
\hline $\begin{array}{l}\text { Understand the priorities of others in } \\
\text { the group }\end{array}$ & 563 & 1 & 5 & 3.95 & .771 \\
\hline $\begin{array}{l}\text { Help others enhance their skills and } \\
\text { abilities }\end{array}$ & 563 & 1 & 5 & 3.87 & .891 \\
\hline $\begin{array}{l}\text { Align disparate viewpoints within the } \\
\text { group }\end{array}$ & 561 & 1 & 5 & 3.74 & .854 \\
\hline
\end{tabular}

Table 1.2.4 Consciousness of Others - Descriptive Statistics 


\section{Gender and Ethnicity}

Thirty-one percent of the sample of college students were male, and $69 \%$ female (Figure 1.2.1). An ethnicity question was asked, with $87 \%$ reporting that they were white (Caucasian), 4\% Hispanic, 3\% Asian/Pacific Islander, 3\% multiracial, 2\% African-American, and 1\% were of Middle Eastern decent

(Figure 1.2.2).

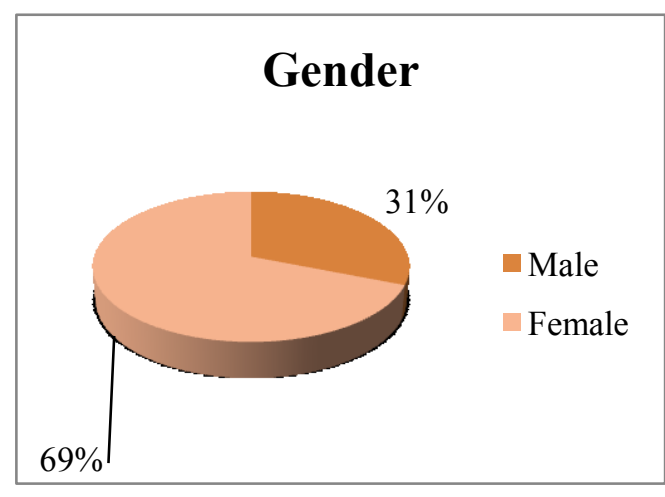

Figure 1.2.1 Respondent gender

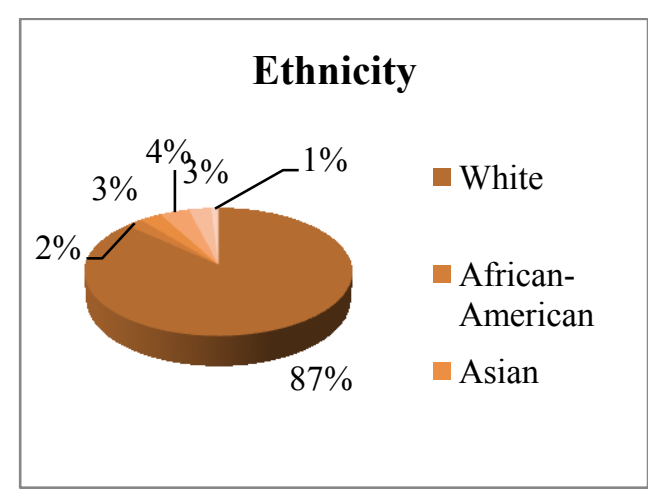

Figure 1.2.2 Respondent ethnicity

\section{Class Rank}

A reasonable distribution of class ranks participated, with $10 \%$ freshman, $28 \%$ sophomores, 32\% juniors, 22\% seniors and 7\% graduate students (Figure 1.2.3).

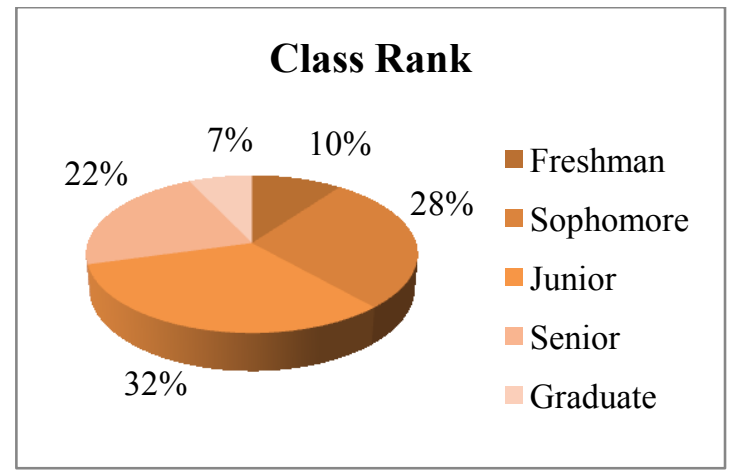

Figure 1.2.3 Percent of respondents in class

\section{Age Category}

Given the varied ages that potentially comprise each class rank, student respondents also provided their age category. Twenty-one percent were 18-19 
years of age, $55 \%$ were $20-21,16 \% 22-23,2 \%$ reported their age group as $24-$ 26 and $6 \%$ were 26 or older (Figure 1.2.4).

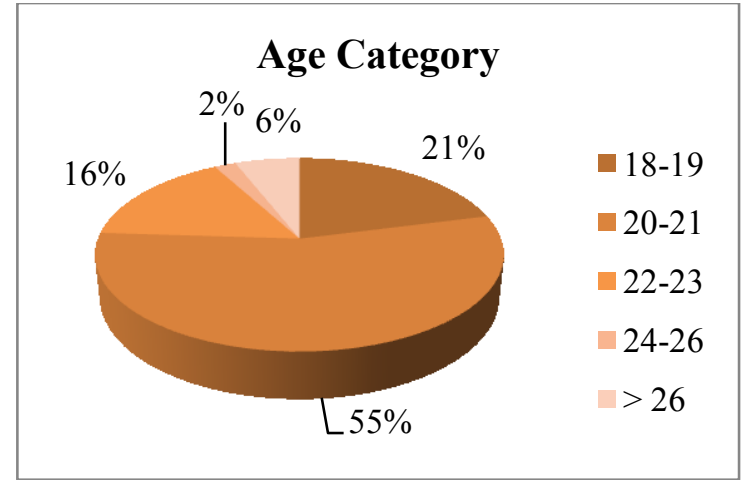

Figure 1.2.4 Respondent percent by age

\section{Student Organization Involvement}

Students were asked to provide the number of campus student organizations in which they were involved. Only 5\% were not involved in any on-campus student organizations, with 54\% involved in at least 3 organizations (Figure 1.2.5).

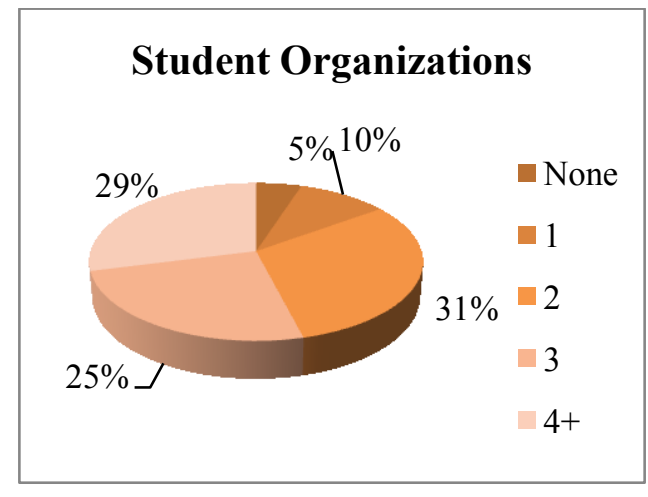

Figure 1.2.5 Student organization involvement leaders

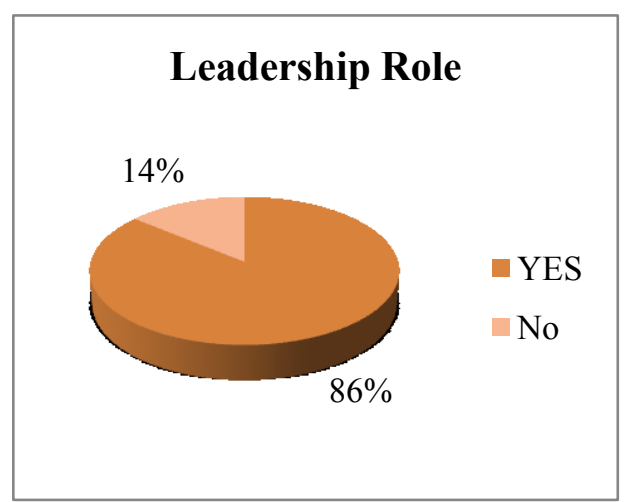

Figure 1.2.6 Percentage student -

Further, $86 \%$ reported that they were currently in some type of leadership role within an organization (Figure 1.2.6).

A final question was asked with regard to number of leadership retreats attended. All but 17\% reported having participated in at least 1 leadership retreat, with nearly $40 \%$ having attended 3 or more. This high-level of involvement in leadership retreats may be somewhat reflective of the purposive sampling conducted in the administration of the survey. Authors 
Shankman and Allen distributed a link to the web-enabled survey primarily to colleagues in universities who in turn distributed to their students.

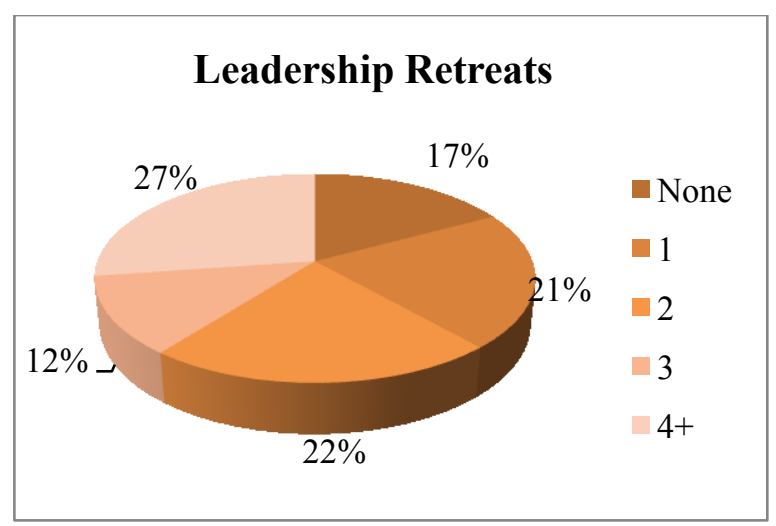

Figure 1.2.7 Participation in leadership retreats

In section 1.4 the more actively involved students will be discussed to better understand the significant differences between these students and their lessinvolved counterparts, as well as gender differences based on level of involvement. 


\subsection{General Problem - Measuring Leadership}

The objective of the work herein is to provide a method to statistically assess an individual's leadership skill set, categorize them in comparison to others, and ultimately, based on the dataset, make suggestions for development.

Emotionally intelligent leadership has multiple definitions, but Shankman and Allen (2009) suggest that the most trusted include:

- "The ability to monitor one's own and others' feelings and emotions, to discriminate among them and to use this information to guide one's thinking and actions" (Salovey \& Mayer, 1990, p. 189).

- Making emotions "work for you by using them to help guide your behavior and thinking in ways that enhance your results" (Weisinger, 1998, p. xvi).

- "The capacity for recognizing our own feelings and those of others, for motivating ourselves, and for managing emotions well in ourselves and in our relationships" (Goleman, 1998, p. 317).

The challenge follows: given the dataset, we seek to assess leadership skills based on self-reported levels of focus on leadership traits and techniques. Then, we need to understand significant differences between higher performing leaders and others, and subsequently utilize those findings to suggest methods for improvement.

There is not a dependent variable, preferably provided from individuals other than the respondents themselves, that offers a measurement of leadership effectiveness. Thus, the analysis is based on discriminating between groups of individuals who self-report levels of consciousness of context, self and others when they are in a leadership role. Additionally, one might consider higher levels of organizational involvement, when coupled with a leadership role, to be indicative of individuals with stronger leadership capacity. The aim is to discern the attributes that set this group apart, and ultimately provide a method for discriminating between these "max-leaders" and others.

There are three main constructs with which to work. Shankman and Allen define consciousness of context as a focus on the environment in which leaders and followers work. Consciousness of self is being aware of oneself in terms of abilities and emotions. Consciousness of others is being aware of one's relationship with others and the role they play in the leadership equation.

Given that the context is the environment in which leaders and followers work (Fiedler, 1996), the settings are often very different, and situations constantly changing, dynamic. People in the organizational context may be open to being 
led, or not. Shankman and Allen suggest that each new context requires a different set of knowledge, skills, and abilities on the part of leaders and followers.

Consciousness of self requires knowing oneself and one's values, as well as how one's actions affect others (Higher Education Research Institute, 1996). Since this is a lifetime effort, Shankman and Allen suggest to their college student audience that it's not important that introspection result in concrete answers, but it is the process that is important. This research aims to help understand that process. Additionally, it must be noted that consciousness of self requires actions that are aligned with one's words. This fosters trust, which is required of leadership (Shankman and Allen, 2003, p. 26).

Consciousness of others requires one to empathize, inspire, influence, coach, manage conflict an effect change. The challenge is in the fact that context is dynamic with infinite number of variables affecting leadership, thus requiring the leader to understand others' communication patterns and preferences, desires, expectations, and work style to name just a few of the affecting variables (ibid, p. 74).

Without delving deep into the leadership "effectiveness" literature, one can safely say the concept is somewhat subjective. Given the context, one's personal goals may be spiritual, monetary, career-oriented, relationshiprelated, familial, etc., each requiring a sense of leadership in order to achieve one's goals effectively. Leadership can be implemented effectively, yet unethically, irresponsibly or immaturely.

Harvard Business School professor Joseph Badaracco suggests that selfreflection is vitally important to leadership, and it's this self-reflection, in our case a specific self-assessment tool, on which this analysis focuses. Badaracco interviewed corporate leaders to understand the tools they used to make decisions for their companies, concluding:

They are able to take time out from the chain of managerial tasks that consumes their time and undertake a process of probing self-inquiry a process that is more often carried out on the run than in quiet seclusion. They are able to dig below the busy surface of their daily lives and refocus on their core values and principles. Once uncovered, those values and principles renew their sense of purpose at work and act as a springboard for shrewd, pragmatic, politically astute action. By repeating this process again and again throughout their work lives, these executives are able to craft an authentic and strong identity based on their own, rather than another's understanding of what is 
right. And in this way, they begin to make the transition from being a manager to becoming a leader.

(Badaracco, HBR, 1998)

In Heroic Leadership, Chris Lowney discusses the 450-year-old traditions of the Jesuits regarding leadership, suggesting leadership development methods must be revisited.

First, no one can make another person self-aware, so leaders must largely mold themselves. Only I can muster the will, courage and honesty to search myself. Others - coaches, managers, friends, parents, and mentors - help of course, but primarily by playing a role similar to that of the "director" in (Ignatius) Loyola's key selfawareness tool, the Spiritual Exercises...All leadership begins with self-leadership, and self-leadership begins with knowing oneself. First comes the foundation: goals and values, an understanding of personal strengths and obstacles, an outlook on the world. Then comes the invigorating daily habit of refreshing and deepening self-knowledge while immersing oneself in a constantly evolving world.

(Lowney, 2003, p.97-98)

These perspectives serve to validate the use of a self-assessment tool as a part of one's personal leadership training and development. 


\subsection{Problem of Classification - Significant Differences on Consciousness of Context, Self and Others}

As in Section 1.5 of Classification and Analysis of a Job Seeking Market, the independent samples t-test procedure is used to identify significant differences between the means of groups of interest.

This section will first discuss the levels created for each construct and the most significant differences between the levels. Gender differences are then investigated and discussed. Also, max-leaders (involved in 3+ organizations, and currently in a leadership role) are investigated as well as the extent to which the attitudes and behaviors of this group significantly differ from the balance of the respondents.

Three levels were created for each construct based on score. Respondents with total scores less than 25 for a given construct were coded as 1, scores between 26 and 34 were coded as level 2, and those in the 35-40 range were coded the highest, at level 3. This same process was used for each of the three consciousness constructs (context, self, and others).

Table 1.4.1 details the number and percentage of respondents falling into each category, and the mean score for the group. Note that most students rated themselves in upper two tiers suggesting a general acceptance of leadership training and development.

\begin{tabular}{|lllll|}
\hline $\begin{array}{l}\text { Consciousness } \\
\text { Construct }\end{array}$ & Level (score) & N & Percent & Mean \\
\hline Context & Level 1 (8-25) & 64 & $11.4 \%$ & 22.91 \\
\hline \multirow{2}{*}{ Self } & Level 2 (26-34) & 388 & $69.3 \%$ & 30.45 \\
\hline & Level 3 (35-40) & 108 & $19.3 \%$ & 37.08 \\
\hline & Level 1 (8-25) & 12 & $2.2 \%$ & 24.17 \\
\hline Others & Level 2 (26-34) & 337 & $62.1 \%$ & 30.96 \\
\hline & Level 3 (35-40) & 194 & $35.7 \%$ & 36.68 \\
\hline & Level 1 (8-25) & 42 & $7.7 \%$ & 23.36 \\
\hline & Level 2 (26-34) & 324 & $59.3 \%$ & 30.78 \\
\hline
\end{tabular}

Table 1.4.1 Levels of consciousness constructs

Figure 1.4.1 is provided as a supplement, to further clarify comparisons of construct scores by levels. 


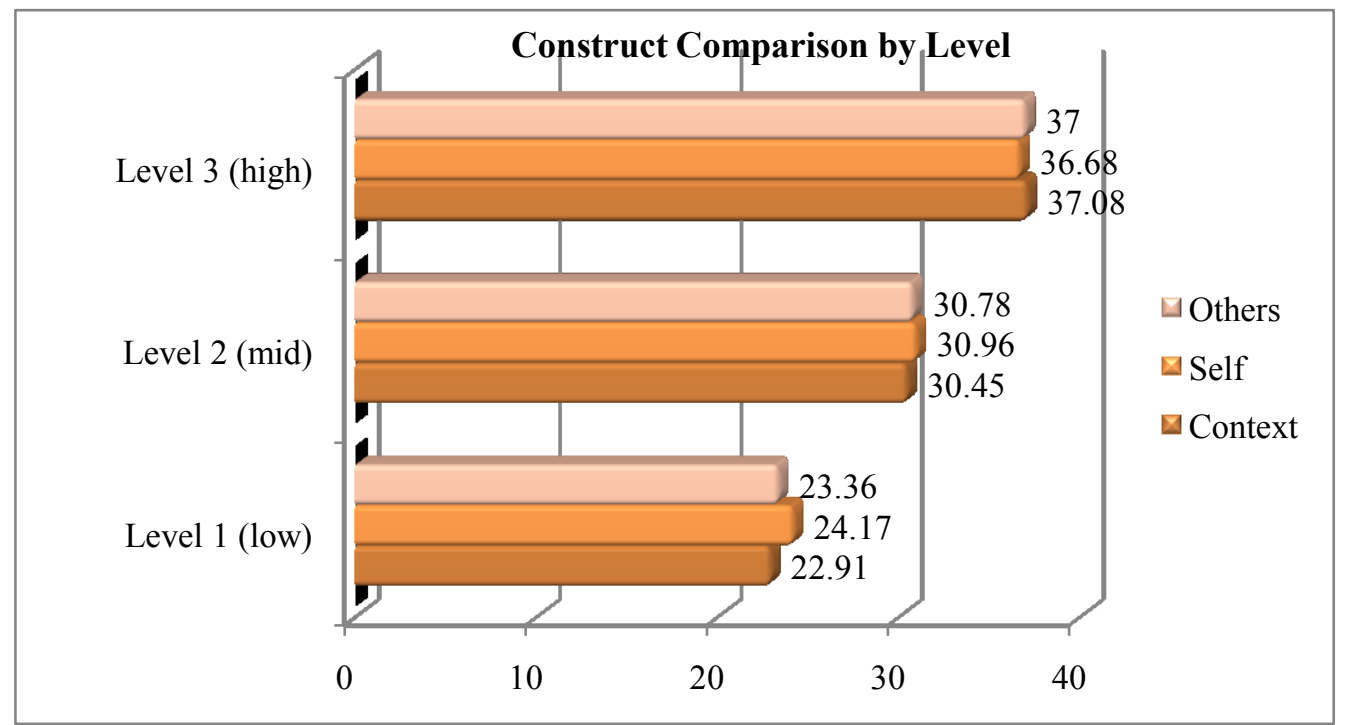

Figure 1.4.1 Construct score comparison by level

As expected, due to the groupings by low, mid and high levels, significant differences are present between variables in each group. It is insightful to note the largest of the mean differences, as these will provide a useful comparison in the discriminant analysis conducted in part two of this study. For now, consider the top three largest mean differences between each of the groups within each of the constructs. Recommendations will be made regarding areas of focus for improving to a higher level.

\section{Consciousness of Context - Largest Mean Differences}

\section{Level 3 (high) vs. Level 2 (mid)}

The highest level leaders (35-40 on consciousness of CONTEXT construct) are significantly more likely than the mid-level leaders to think about how their leadership style aligns with the group's culture. They are more likely to understand how the group's environment influences their leadership style and subsequently tailor their style to the leadership situation. Essentially, these are elements that mid-level leaders can focus on for development of consciousness of context.

Interestingly, the mid-level group's mean rating for thinking about how their leadership style aligns with group culture was rated second-lowest of the 24 attributes measured, suggesting a key area for consideration and improvement by the mid-level group. 


\begin{tabular}{|lllllll|}
\hline $\begin{array}{l}\text { When serving in a formal } \\
\text { or informal leadership } \\
\text { role, I... }\end{array}$ & $\bar{x}_{\text {high }}$ & $\bar{x}_{\text {mid }}$ & $\begin{array}{c}\text { Mean } \\
\text { diff. }\end{array}$ & $\boldsymbol{t}$ & df & $p$ \\
$\begin{array}{l}\text { Think about how my } \\
\text { leadership style aligns with } \\
\text { group culture }\end{array}$ & 4.60 & 3.59 & 1.01 & 14.59 & 219 & .000 \\
\hline $\begin{array}{l}\text { Understand how the group's } \\
\text { environment influences my } \\
\text { leadership style }\end{array}$ & 4.60 & 3.68 & .921 & 13.61 & 220 & .000 \\
\hline $\begin{array}{l}\text { Tailor my leadership style to } \\
\text { the situation }\end{array}$ & 4.76 & 3.92 & .837 & 14.13 & 274 & .000 \\
\hline
\end{tabular}

Table 1.4.2 CONTEXT Level 3 (high) vs. Level 2 (mid)

\section{Level 2 (mid) vs. Level 1 (low)}

Next, compare the mid-level leaders on consciousness of CONTEXT to the lower group. The mid-level group is significantly more likely to understand how the group members relate to one another. They tend to a greater understanding of how the group's environment influences their leadership style, and they are more likely to think about how their leadership style aligns with the group culture, which again, is a focus integral to continued development.

The students in the lowest level group rated this variable dead last as far as their leadership behaviors regarding consciousness of CONTEXT, perhaps because they may still be unclear as to what their "leadership style" is or what options they have for aligning it with group culture.

\begin{tabular}{|c|c|c|c|c|c|c|}
\hline $\begin{array}{l}\text { When serving in a formal or } \\
\text { informal leadership role, I... }\end{array}$ & $\bar{x}_{m i d}$ & $\bar{x}_{\text {low }}$ & $\begin{array}{l}\text { Mean } \\
\text { diff. }\end{array}$ & $t$ & df & $p$ \\
\hline $\begin{array}{l}\text { Understand how the group } \\
\text { members relate to one another }\end{array}$ & 3.91 & 2.80 & 1.16 & 9.91 & 76 & .000 \\
\hline $\begin{array}{l}\text { Understand how the group's } \\
\text { environment influences my } \\
\text { leadership style }\end{array}$ & 3.68 & 2.66 & 1.02 & 9.86 & 450 & 000 \\
\hline $\begin{array}{l}\text { Think about how my leadership } \\
\text { style aligns with group culture }\end{array}$ & 3.59 & 2.62 & .965 & 9.09 & 450 & 000 \\
\hline
\end{tabular}

\section{Consciousness of SELF}

Level 3 (high) vs. Level 2 (mid)

Considering consciousness of SELF, level 3 leaders are significantly more likely to work on their limitations compared to their level 2 counterparts. 
They intend to improve on their abilities, and they are more reflective regarding how their actions align with their values. A focus on these behaviors provides a foundation for development for the mid-level group to improve consciousness of self when in a leadership role.

Here again, as seen earlier with students at low-level consciousness of CONTEXT and their lack of focus on aligning their leadership style to the group's culture, the mid-level group is rating their propensity to work on their limitations at the very bottom of the list of behaviors. At 3.19, it is still slightly more than "sometimes" if we consider the 5-point scale, but it is still the lowest rated behavior suggesting a key area of focus for development of leadership skills with regard to consciousness of SELF.

\begin{tabular}{|lcccccc|}
$\begin{array}{l}\text { When serving in a } \\
\text { formal or informal } \\
\text { leadership role, I... }\end{array}$ & $\bar{x}_{\text {high }}$ & $\bar{x}_{\text {mid }}$ & Mean diff & $\boldsymbol{t}$ & df & $\boldsymbol{p}$ \\
\hline $\begin{array}{l}\text { Work on my limitations } \\
\text { Improve my abilities }\end{array}$ & 4.14 & 3.19 & .952 & 14.21 & 529 & .000 \\
\hline $\begin{array}{l}\text { Reflect on how my } \\
\text { actions align with } \text { my } \\
\text { values }\end{array}$ & 4.62 & 3.80 & .831 & 15.65 & 529 & .000 \\
\hline
\end{tabular}

Table 1.4.4 SELF Level 3 (high) vs. Level 2 (mid)

Level 2 (mid) vs. Level 1 (low)

Comparing the level 2 group to the lowest level on the consciousness of SELF construct, the mid-level is significantly more likely to be working on improving their abilities when in a leadership role. They are more reflective regarding how their actions align with their values and are significantly more likely to establish a positive tone.

The lowest level group rated "improve my abilities" second last, at 2.50, halfway between infrequently and sometimes, suggesting a key area of focus for improvement toward a higher level of consciousness of SELF when in a leadership role. Aligning actions with values is a somewhat more significant result (although the mean difference is not as large) thus additional focus in this area is recommended to yield improvement.

\begin{tabular}{|c|c|c|c|c|c|c|}
\hline $\begin{array}{l}\text { When serving in a } \\
\text { formal or informal } \\
\text { leadership role, I... }\end{array}$ & $\bar{x}_{m i d}$ & $\bar{x}_{\text {low }}$ & Mean diff. & $t$ & df & $p$ \\
\hline Improve my abilities & 3.80 & 2.50 & 1.29 & 4.47 & 11 & .001 \\
\hline $\begin{array}{l}\text { Reflect on how my } \\
\text { actions align with } \mathbf{m y} \\
\text { values }\end{array}$ & 3.82 & 2.67 & 1.15 & 5.16 & 347 & .000 \\
\hline Establish a positive tone & 4.10 & 3.08 & 1.01 & 5.01 & 347 & .000 \\
\hline
\end{tabular}

Table 1.4.5 SELF Level 2 (mid) vs. Level 1 (low) 


\section{Consciousness of OTHERS}

\section{Level 3 (high) vs. Level 2 (mid)}

High-level leaders are significantly more conscious of helping others enhance their skills and abilities when in a leadership role. These leaders are significantly more likely to work to resolve conflicts than the mid-level group, and they tend to a greater focus on understanding the priorities of others in the group.

The mid-level group rated their propensity to help others enhance their skills and abilities at 3.63, slightly more than half-way between sometimes and frequently behaving in this manner, suggesting a key area for improvement.

\begin{tabular}{|c|c|c|c|c|c|c|}
\hline $\begin{array}{l}\text { When serving in a formal } \\
\text { or informal leadership role, } \\
\text { I... }\end{array}$ & $\bar{x}_{h i g h}$ & $\bar{x}_{\text {mid }}$ & $\begin{array}{l}\text { Mean } \\
\text { diff. }\end{array}$ & $t$ & df & $p$ \\
\hline $\begin{array}{l}\text { Help others enhance their } \\
\text { skills and abilities }\end{array}$ & 4.55 & 3.63 & .923 & 15.35 & 440 & .000 \\
\hline $\begin{array}{l}\text { Work to resolve conflicts } \\
\text { within group }\end{array}$ & 4.81 & 3.96 & .846 & 17.01 & 497 & .000 \\
\hline $\begin{array}{l}\text { Understand priorities of } \\
\text { others in group }\end{array}$ & 4.53 & 3.76 & .765 & 13.47 & 502 & .000 \\
\hline
\end{tabular}

\section{Level 2 (mid) vs. Level 1 (low)}

Differentiating mid-level leaders versus the lowest level on consciousness of others, it is clear that mid-level leaders tend to a higher likelihood of thinking about how their decisions are received by others in the group. Further, they listen more carefully to what is and is not being said in the group setting. Also, the mid-level group is significantly more likely to work to resolve conflicts within the group.

Following suit, the behavior with the most significant difference, thinking about how one's decisions are received, was rated $4^{\text {th }}$ from the bottom on the list of twenty-four behavioral focuses when in a leadership role. A greater concentration on this skill is likely to help the low-level scorers move up to the mid-level. 


\begin{tabular}{|lcccccc|}
\hline $\begin{array}{l}\text { When serving in a formal } \\
\text { or informal leadership } \\
\text { role, I... }\end{array}$ & $\bar{x}_{\text {mid }}$ & $\bar{x}_{\text {low }}$ & $\begin{array}{l}\text { Mean } \\
\text { diff. }\end{array}$ & $\boldsymbol{t}$ & df & $\boldsymbol{p}$ \\
$\begin{array}{l}\text { Think about how my } \\
\text { decisions are received by } \\
\text { group members }\end{array}$ & 3.92 & 2.81 & 1.11 & 9.31 & 364 & .000 \\
\hline $\begin{array}{l}\text { Listen carefully to what is } \\
\text { and isn't being said }\end{array}$ & 3.97 & 2.86 & 1.11 & 7.11 & 47 & .000 \\
\hline $\begin{array}{l}\text { Work to resolve conflicts } \\
\text { within group }\end{array}$ & 3.96 & 2.86 & 1.10 & 7.46 & 47 & .000 \\
\hline
\end{tabular}

Table 1.4.7 OTHERS Level 2 (mid) vs. Level 1 (low)

In summary, students with scores at the low and mid levels of consciousness of CONTEXT, should primarily focus on thinking about how their leadership style aligns with the group culture. This implies a potential need for effective training in this area so student understand what their leadership style actually is, and how to adapt it to the situation or context.

Concerning consciousness of SELF, mid-level leaders need to concentrate on working on their limitations, while the lowest level group should focus on improving their abilities.

A focus on helping others enhance their skills and abilities is likely to take students at the mid-level of consciousness of OTHERS to a higher level. Lastly, a stronger focus on thinking about how one's decisions are received is integral to improvement for those ranking in the lowest level of consciousness of OTHERS.

\section{Consciousness Compared by Level of Involvement}

Next, consider only those who are involved in at least one student organization. This eliminates only $5 \%$ of the sample, yet allows for comparisons by varied levels of involvement. This analytical step is certainly justified because significant differences are evidenced on all three constructs when those involved in at least one student organization are compared to those not involved in any (Figure 1.4.2). The largest mean difference, 3.89 is with consciousness of CONTEXT, followed by SELF at 3.29 and OTHERS at 2.77, clearly suggesting that involvement in student organizations serves to enhance students' perceptions of the leadership consciousness constructs. 


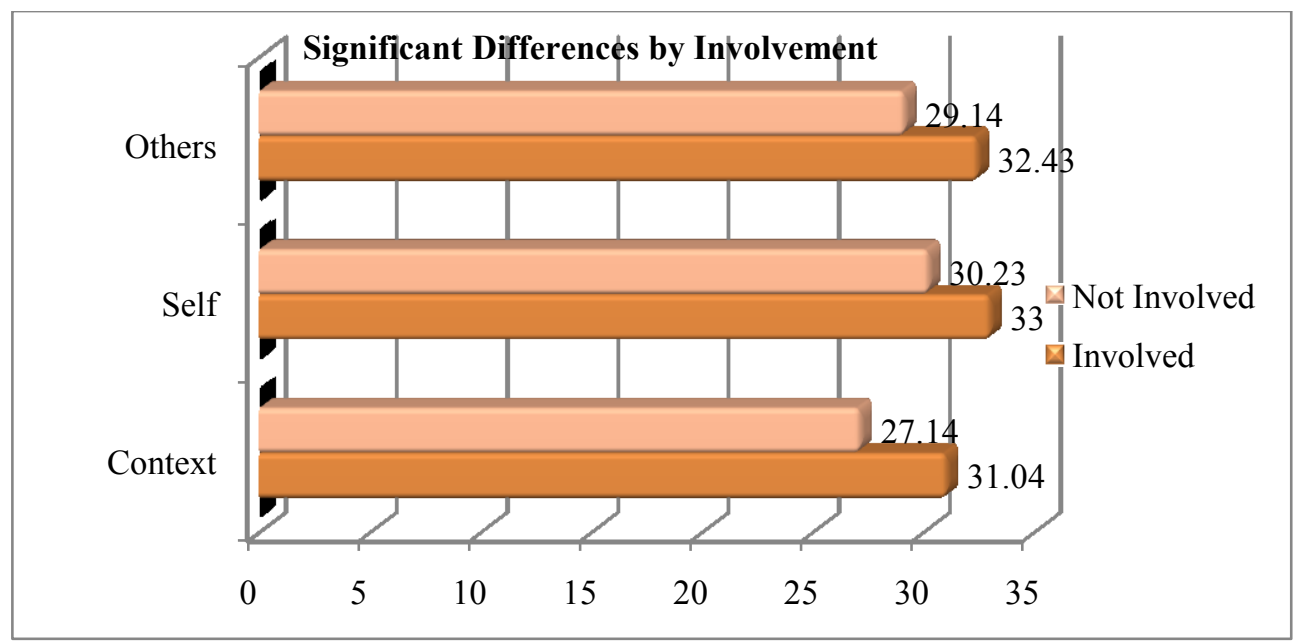

Figure 1.4.2 Comparison by involvement in at least 1 student organization; significant differences evidenced on all three constructs

Investigating the three consciousness constructs for significant differences based on level of involvement, regardless of gender at this point, the impact is found at the $4+$ level of organizational involvement versus fewer. In other words, people involved in 4 or more organizations are significantly more conscious of CONTEXT, SELF, and OTHERS compared to those involved in fewer organizations. Looking deeper one finds the point at which that consciousness "kicks in". It is found at the 3 organization involvement level. So, there is a significant difference on all three constructs once the student is involved in at least one organization, then no significant change is evidenced until the student hits the 4+ involvement category, where there are significant differences in consciousness of SELF and OTHERS between those involved in 3 groups versus four or more. This $4+$ group rates significantly higher on SELF (33.64) and OTHERS (33.04), compared to those involved in 3 groups, where the mean score for SELF was 32.43 and others 31.82 (with $\mathrm{t}=2.96$ and 2.41 respectively).

Note that CONTEXT has dropped from significance but only because it was negligibly close to the threshold $(\mathrm{t}=1.994, \mathrm{p}=.047)$ when compared to the larger group of all those in less than 4 groups, instead of just those involved in 3 groups where the difference in consciousness of context is insignificant. Table 1.4.9 summarizes the differences discussed above.

\section{Max-Leaders - Significant Differences}

As noted at the end of Section 1.2, the description of the dataset, a separate category was created for student respondents who participated in 3 or more student organizations, and were currently in a leadership role. Hereafter, this group is referred to as "max-leaders" although it should be noted that it is not 
suggested that maximum activity necessarily implies leadership effectiveness. It is, however, worthwhile to investigate the significant differences between these high-activity student respondents versus their less-active counterparts, which are referred to as the base group. Max-leaders are significantly more conscious of CONTEXT, rated at 31.28 versus the balance of the respondents (base) at 30.44 (Figure 1.4.3).

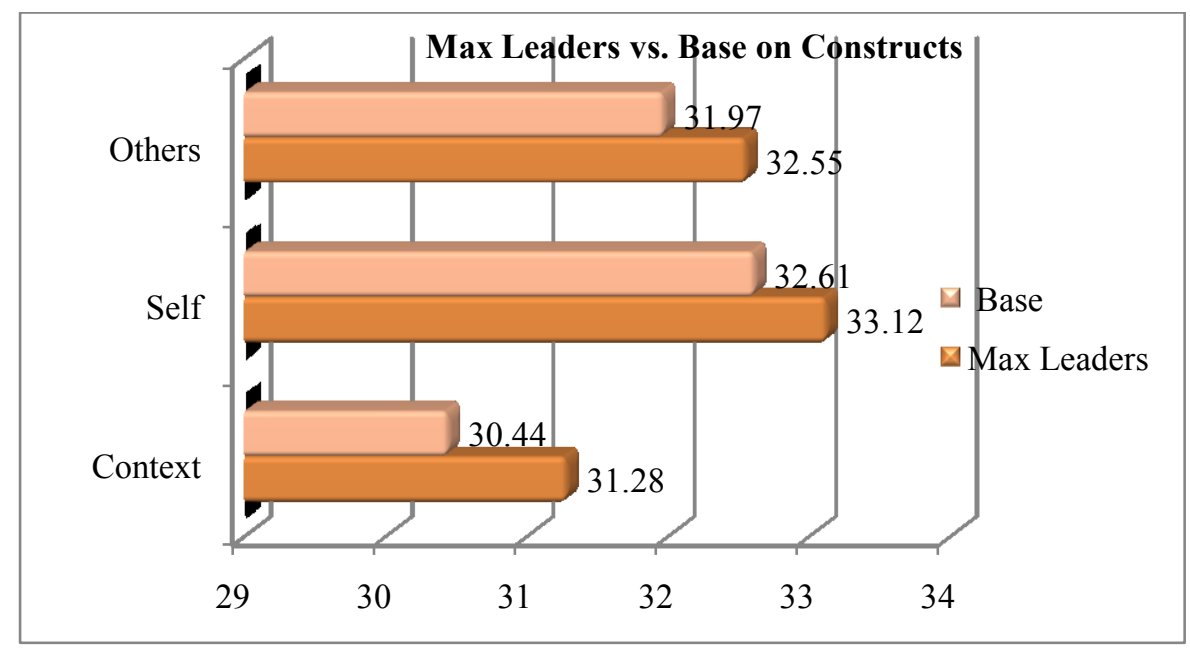

Figure 1.4.3 Max leaders scored significantly higher on consciousness of context.

This may be due to their more active involvement in student organizations (three or more) versus those not in the max-leader category, participating in two or fewer organizations. Of those in the base group $61 \%$ participate in two groups, $20 \%$ in one group. Ten percent are not involved in any student organizations, and another $9 \%$ participate in three or more groups, but are not in a leadership role, thus not qualifying for "max-leader" status.

Consider the significant differences between max-leaders and the base group on all leadership behaviors measured. Max-leaders are significantly more likely to take time to understand the informal traditions of the group, a consciousness of CONTEXT variable. Further, they are more accustomed to working with others toward a shared goal (SELF). Lastly, max-leaders put others first, significantly more so, by focusing on enhancing others' skills and this is a consciousness of OTHERS variable (Table 1.4.8). 


\begin{tabular}{|llllllll|}
\hline $\begin{array}{l}\text { When serving in a } \\
\text { formal or informal } \\
\text { leadership role, I... }\end{array}$ & Construct & $\bar{x}_{\text {max }}$ & $\bar{x}_{\text {base }}$ & $\begin{array}{l}\text { Mean } \\
\text { diff. }\end{array}$ & $\boldsymbol{t}$ & df & $\boldsymbol{p}$ \\
$\begin{array}{l}\text { Take time to } \\
\text { understand informal } \\
\text { traditions }\end{array}$ & CONTEXT & 3.95 & 3.73 & .211 & 3.07 & 560 & .002 \\
\hline $\begin{array}{l}\text { Work with others } \\
\text { toward a shared goal }\end{array}$ & SELF & 4.44 & 4.28 & .158 & 2.73 & 560 & .006 \\
\hline $\begin{array}{l}\text { Help others enhance } \\
\text { skills and abilities }\end{array}$ & OTHERS & 3.95 & 3.79 & .157 & 2.10 & 561 & .036 \\
\hline
\end{tabular}

Table 1.4.8 Max Leaders vs. base - Significant Differences

Max-leaders appear to benefit from leadership positions and high levels of involvement, particularly as it relates to consciousness of context. Their significantly different behaviors appear to indicate multiple focuses because each of the consciousness constructs are represented. This suggests that a balanced sense of consciousness is required by those highly involved in organizations, and simultaneously maintaining leadership roles.

\section{Gender and Involvement}

The primary focus of this portion of the research is to explore the role that gender plays in college students' perceptions of the three facets of emotionally intelligent leadership (consciousness of context, consciousness of self, and consciousness of others). Additionally, this portion examines gender differences in students' emotionally intelligent leadership based on students' levels of involvement in campus organizations. This suggests the following two research questions:

1. What, if any, gender differences exist in students' consciousness of context, consciousness of self, and consciousness of others?

2. What, if any, gender differences exist in students' consciousness of context, consciousness of self, and consciousness of others based on level of involvement?

Looking at involvement by gender, $12 \%$ of men were involved in one organization, $27 \%$ in two organizations, $25 \%$ in three organization, and $34 \%$ in four or more organizations. Ten percent of the women were involved in one organization, $34 \%$ in two organizations, $27 \%$ in three organizations, and $29 \%$ in four our more organizations. 


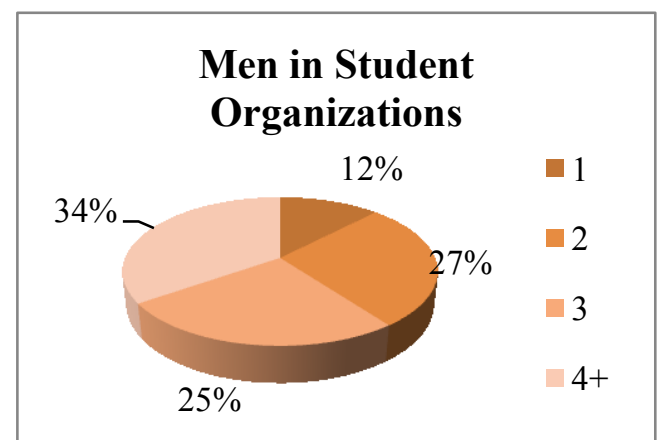

Figure 1.4.4 Men in student organizations

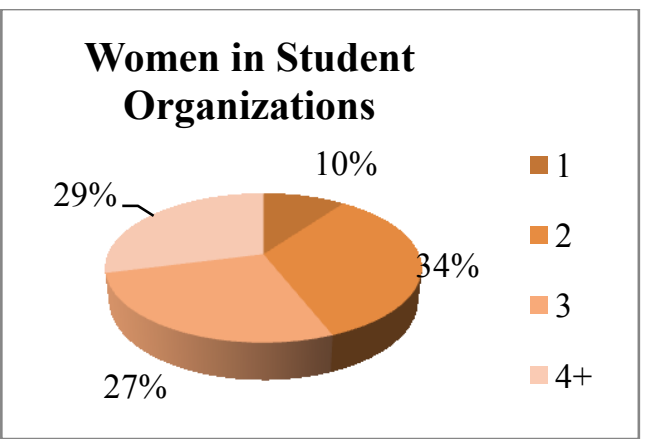

Figure 1.4.5 Women in student organizations

Research question one read: What, if any, gender differences exist in students' consciousness of context, consciousness of self, and consciousness of others?

Gender results for mean differences indicated significant differences between men and women on both consciousness of self and consciousness of others. Consciousness of self was significantly higher for women at 33.08 as compared to men at $32.33(t=2.25)$. Consciousness of others was also significantly higher for women at 32.56 as compared to men at $31.64(t$ $=2.16$ ). No significant differences by gender were identified between the mean ratings on the consciousness of context construct.

Table 1.4.9 highlights the results for specific measures in which significant differences were identified between the means of the gender groups. Three of the measures load on the consciousness of others construct, and three load on the consciousness of self construct. Women appear to demonstrate greater consideration of others in the group and think about how their decisions are received by others. Additionally, women tend to monitor how their emotions affect their interactions (self) more so than men, and they place a significantly stronger focus on following through on commitments (self). Women also demonstrate a greater likelihood to reflect on how their actions align with their values (self), and they listen more carefully to what is said and unsaid (others). The only variable in which men demonstrated higher mean scores than women was capitalization on strengths (self). 


\begin{tabular}{|c|c|c|c|c|c|c|c|}
\hline $\begin{array}{l}\text { When serving in a } \\
\text { formal or informal } \\
\text { leadership role, I... }\end{array}$ & Construct & $\bar{x}_{\text {women }}$ & $\bar{x}_{m e n}$ & $\begin{array}{l}\text { Mean } \\
\text { diff }\end{array}$ & $t$ & df & $p$ \\
\hline $\begin{array}{l}\text { Consider needs of } \\
\text { others in the group }\end{array}$ & Others & 4.32 & 4.09 & .23 & 3.47 & 315 & .001 \\
\hline $\begin{array}{l}\text { Monitor how my } \\
\text { emotions affect my } \\
\text { interactions with others }\end{array}$ & Self & 3.99 & 3.79 & .20 & 2.41 & 558 & .010 \\
\hline $\begin{array}{l}\text { Think about how my } \\
\text { decisions are received }\end{array}$ & Others & 4.13 & 3.94 & .19 & 2.60 & 319 & .016 \\
\hline $\begin{array}{l}\text { Follow through on } \\
\text { commitments }\end{array}$ & Self & 4.52 & 4.33 & .19 & 3.11 & 303 & .002 \\
\hline $\begin{array}{l}\text { Reflect on how my } \\
\text { actions align with my } \\
\text { values }\end{array}$ & Self & 4.13 & 3.97 & .16 & 2.16 & 558 & .031 \\
\hline $\begin{array}{l}\text { Listen carefully to what } \\
\text { is and isn't being said }\end{array}$ & Others & 4.15 & 3.99 & .16 & 2.01 & 559 & .045 \\
\hline $\begin{array}{l}\text { Capitalize on my } \\
\text { strengths }\end{array}$ & Self & 4.08 & 4.23 & -.15 & -2.24 & 558 & .026 \\
\hline
\end{tabular}

Table 1.4.9 Gender Differences for individual measures from research question one

In examining those respondents who scored in the highest levels across all three constructs of consciousness (between $35-40$ on each, 13\% of total respondents), there was only one significant difference by gender. Women reported significantly higher scores (4.93) than men (4.56) for the measure of "consider the needs of others in the group" $(\mathrm{t}=3.01)$. Even among the students with the highest levels of self-reported leadership behaviors, women tend to consider the needs of others in the group more often than men.

Research question two reads: What, if any, gender differences exist in students' consciousness of context, self and others, based on level of involvement?

When gender differences within the three constructs were examined in relation to respondents' level of involvement, a few significant differences emerged. Similar to findings from research question one, there were no significant differences by gender for the construct of consciousness of context. Significant differences by gender did exist, though, for consciousness of self and consciousness of others when taking into consideration involvement. Additionally, there were many significant differences by gender across all levels of involvements for individual measures of consciousness of context, self, and others. The findings on gender differences for the individual measures are summarized in Table 1.4.10. 
For students involved in only one organization, significant differences by gender were minimal. One significant difference emerged within this group of less-involved students for the consciousness of self measure of capitalizing on strengths, with mean scores of 4.53 for men compared to 4.03 for women $(\mathrm{t}=$ 2.46). No significant differences were evident by gender for the three constructs.

For respondents involved in two student organizations, women scored significantly higher on the construct of consciousness of self than men (33.21 vs. 31.54; $\mathrm{t}=2.74)$. The specific measure in which gender differences were particularly evident was following through on commitments (4.51 for women and 4.21 for men; $t=2.68$ ). While significant differences were not evident on the other two facets (others and context) for the students involved in two organizations, there are some interesting differences for some of the specific measures. Women involved in two organizations are significantly more likely to work on building a sense of team (others), rating themselves at 4.15 compared to men at $3.80(\mathrm{t}=2.61)$. They are also more likely to consider the needs of others in the group (4.34) as compared to men (4.07; $t=2.15)$. Last, one of the measures of consciousness of context was significant; women are more likely to recognize patterns of behavior in the group (4.11 vs. 3.78 ; $\mathrm{t}=$ 2.60).

For respondents involved in three student organizations, women (32.35) were significantly higher in the construct of consciousness of others than men ( 32.35 for women and 30.55 for men; $\mathrm{t}=2.66$ ). Women in three organizations were significantly more likely to consider others' needs (4.30 vs. 3.92; $\mathrm{t}=2.73$ ), think about how their decisions were received by the group (4.09 vs. $3.75 ; \mathrm{t}=2.19$ ), and try to understand the priorities of others in the group versus their male counterparts $(4.0$ vs. $3.69 ; \mathrm{t}=2.24)$. All three are measures within the consciousness of others construct.

For those involved in at least four organizations, there were no significant differences by gender in the three overall consciousness constructs. For individual measures, though, there were a few significant differences. Men were significantly more likely to work on their limitations (3.83 vs. 3.48), align disparate viewpoints within the group (3.93 vs. 3.64), (others), and capitalize on their strengths (4.38 vs. 4.11) as compared to women. 


\begin{tabular}{|c|c|c|c|c|c|c|c|c|}
\hline $\begin{array}{l}\text { Number of } \\
\text { Organiza- } \\
\text { tions }\end{array}$ & $\begin{array}{l}\text { When serving in a } \\
\text { formal or informal } \\
\text { leadership role, I... }\end{array}$ & Construct & $\bar{x}_{\text {women }}$ & $\bar{x}_{m e n}$ & $\begin{array}{l}\text { Mean } \\
\text { diff. }\end{array}$ & $t$ & df & $p$ \\
\hline One & $\begin{array}{l}\text { Capitalize on my } \\
\text { strengths }\end{array}$ & Self & 4.03 & 4.53 & -.50 & -2.46 & 56 & .017 \\
\hline \multirow[t]{4}{*}{ Two } & $\begin{array}{l}\text { Building a sense of } \\
\text { team }\end{array}$ & Others & 4.15 & 3.80 & .35 & 2.61 & 167 & .010 \\
\hline & $\begin{array}{l}\text { Recognize patterns of } \\
\text { behaviors in the } \\
\text { group }\end{array}$ & Context & 4.11 & 3.78 & .33 & 2.60 & 169 & .010 \\
\hline & $\begin{array}{l}\text { Follow through on } \\
\text { commitments }\end{array}$ & Self & 4.51 & 4.21 & .30 & 2.68 & 169 & .008 \\
\hline & $\begin{array}{l}\text { Consider needs of } \\
\text { others in the group }\end{array}$ & Others & 4.34 & 4.07 & .27 & 2.15 & 169 & .033 \\
\hline \multirow[t]{3}{*}{ Three } & $\begin{array}{l}\text { Consider needs of } \\
\text { others in the group }\end{array}$ & Others & 4.30 & 3.92 & .38 & 2.73 & 136 & .007 \\
\hline & $\begin{array}{l}\text { Think about how my } \\
\text { decisions are received }\end{array}$ & Others & 4.09 & 3.75 & .34 & 2.19 & 136 & .030 \\
\hline & $\begin{array}{l}\text { Understand priorities } \\
\text { of others in group }\end{array}$ & Others & 4.00 & 3.69 & .31 & 2.24 & 134 & .027 \\
\hline \multirow[t]{3}{*}{$\begin{array}{l}\text { Four or } \\
\text { More }\end{array}$} & $\begin{array}{l}\text { Work on my } \\
\text { limitations }\end{array}$ & Self & 3.48 & 3.83 & -.35 & -2.25 & 155 & .026 \\
\hline & $\begin{array}{l}\text { Align disparate } \\
\text { viewpoints within } \\
\text { group }\end{array}$ & Others & 3.64 & 3.93 & -.29 & -2.07 & 158 & .040 \\
\hline & $\begin{array}{l}\text { Capitalize on my } \\
\text { strengths }\end{array}$ & Self & 4.11 & 4.38 & -.27 & -2.27 & 157 & .025 \\
\hline
\end{tabular}

Table 1.4.10 Gender differences for individual measures based on level of involvement

Next, this work transitions to the application of analytic methods to better understand the reliability of the self-assessment tool used to determine a college student's leadership behaviors. Classification methods will be demonstrated with discriminant analysis. Cluster analysis is also discussed which proves to be a worthwhile method, yielding reliable results and valuable insights into the assessment of college student leadership behaviors. 


\subsection{Reliability of Consciousness Constructs and Factor Analysis}

To better understand the constructs of consciousness, both reliability analysis and factor analysis were implemented. In reliability analysis, Cronbach's alpha is the primary criterion to be examined. For the factor analysis, principal components analysis was the extraction method, and Varimax the rotation method, with Kaiser normalization (for more details, see Section 2.1 in Job Seeker Classification and Analysis).

Reliability of Constructs as Scales of Measurement

Several iterations of construct development led to breaking down the original 24 variables included in the assessment of emotionally intelligent leadership, to three constructs considered as reflective of consciousness of context, consciousness of self, and consciousness of others. Table 2.1.1 through Table 2.1.3 supply the variables comprising each construct.

\begin{tabular}{l} 
Consciousness of CONTEXT \\
Take time to understand the informal traditions of the group \\
\hline Learn the expressed and implicit values of the group \\
\hline Tailor my leadership style to the situation \\
\hline Understand how the group's environment influences my leadership style \\
\hline Understand how the group members relate to one another \\
\hline Think about how my leadership style aligns with group culture \\
\hline Identify external influences on the group \\
\hline Recognize the patterns of behavior in the group
\end{tabular}

Table 2.1.1 Variables comprising consciousness of context construct

\begin{tabular}{|l|}
\hline Consciousness of SELF \\
Monitor how my emotions affect my interactions with others \\
\hline Work on my limitations \\
\hline Capitalize on my strengths \\
\hline Follow through on my commitments \\
\hline Establish a positive tone \\
\hline Improve my abilities \\
\hline Reflect on how my actions align with my values \\
\hline Work with others toward a shared goal \\
\hline
\end{tabular}

Table 2.1.2 Variables comprising consciousness of self construct 


\begin{tabular}{|l|}
\hline Consciousness of OTHERS \\
Help others enhance their skills and abilities \\
\hline Work to build a sense of team \\
\hline Think about how my decisions are received by group members \\
\hline Consider the needs of others in the group \\
\hline Understand the priorities of others in the group \\
\hline Listen carefully to what is and isn't being said by group members \\
\hline Work to resolve conflicts within the group \\
\hline Align disparate viewpoints within the group \\
\hline
\end{tabular}

Table 2.1.3 Variables comprising consciousness of others construct

Reliability of the assessment tool is ensured in that the constructs (also referred to as scales), comprised of 8 questions each for simplicity of student self-scoring, achieve strong levels of reliability as measured by Cronbach's alpha coefficient. (Table 2.1.4).

\begin{tabular}{|lcl|} 
FACTOR & Cronbach's Alpha & Sample size \\
\hline Consciousness of Context & $\alpha=.81$ & $\mathrm{n}=560$ \\
\hline Consciousness of Self & $\alpha=.73$ & $\mathrm{n}=543$ \\
\hline Consciousness of Others & $\alpha=.82$ & $\mathrm{n}=546$ \\
\hline
\end{tabular}

Table 2.1.4 Reliability of factors

The goal of each scale (consciousness of context, consciousness of self, consciousness of others) is to use several questions that are consistent in measuring the construct, yet each must contribute unique information. The coefficient, $\alpha$, is based on the number of items in the scale and the ratio of average inter-item covariance to the average item variance (Cronbach, 1951). As the number of items, or questions, increases, the measurement of Cronbach's alpha is likely to increase. If the average inter-item correlation is low, alpha will be low. As the average inter-item correlation increases, Cronbach's alpha increases as well. Typically, 0.60 to 0.80 indicates acceptable reliability, 0.80 or higher means good reliability, and coefficients greater than 0.95 may indicate redundancy in the questions. Garson (2009) suggests the widely accepted cut-off in the social sciences is 0.70 with some researchers using 0.75 to 0.80 . Others are as lenient as 0.60 . Garson notes that 0.70 is as low as one should go because at that point the standard error if measurement will be over half $(0.55)$ of a standard deviation. 
Factor Analysis - College Student Leadership Data

Factor analysis yields interesting results, with a total of five factors explaining nearly $53 \%$ of the variance $(52.6 \%$, based on eigenvalues greater than $1, n=$ 546). The factor loadings from the rotated component matrix reveal important insights, particularly regarding the "interaction" of SELF and OTHER variables on the first component which explains the most (14.29\%) variance. The second component maintains the concept of consciousness of context, and explains an additional $13.18 \%$ of the variance; with the third component offering another $10.82 \%$, the fourth $8.35 \%$ and the fifth component $6.15 \%$. Table 2.1.5 provides the rotated component score matrix with loadings highlighted in matching colors.

Rotated Component Matrix ${ }^{\mathrm{a}}$

\begin{tabular}{|c|c|c|c|c|c|}
\hline & \multicolumn{5}{|c|}{ Component } \\
\hline & 1 & 2 & 3 & 4 & 5 \\
\hline 1. Take time to understand the informal traditions of the group & .221 & .704 & .058 & -.036 & .032 \\
\hline 4. Learn the expressed and implicit values of the group & .094 & 689 & .139 & .131 & .159 \\
\hline 5. Monitor how my emotions affect my interactions with others & .064 & .180 & .264 & .649 & -.094 \\
\hline 6. Work on my limitations & .054 & .091 & .580 & .449 & .057 \\
\hline 7. Tailor my leadership style to the situation & .216 & .137 & .702 & .150 & .127 \\
\hline 8. Capitalize on my strengths & .134 & 156 & .602 & -.112 & .452 \\
\hline $\begin{array}{l}\text { 9. Understand how the group's environment influences my } \\
\text { leadership style }\end{array}$ & 198 & .350 & .577 & .171 & -.029 \\
\hline 11. Help others enhance their skills and abilities & .455 & .377 & .351 & .057 & .024 \\
\hline 12. Understand how the group members relate to one another & .239 & .549 & .186 & .199 & .071 \\
\hline 13. Follow through on my commitments & .224 & .027 & .064 & .014 & .756 \\
\hline 14.Work to build a sense of team & .631 & .276 & .210 & .012 & .146 \\
\hline $\begin{array}{l}\text { 15. Think about how my leadership style aligns with group } \\
\text { culture }\end{array}$ & .255 & .498 & .364 & .125 & -.050 \\
\hline 16. Establish a positive tone & .688 & -.043 & .048 & .330 & .061 \\
\hline $\begin{array}{l}\text { 17. Think about how my decisions are received by group } \\
\text { members }\end{array}$ & .521 & .164 & .176 & .420 & -.015 \\
\hline 18. Improve my abilities & .378 & 159 & .411 & .220 & .249 \\
\hline 20. Identify external influences on the group & .029 & .575 & .258 & .246 & .060 \\
\hline 22. Consider the needs of others in the group & .421 & .169 & -.019 & .534 & .298 \\
\hline 23. Recognize the patterns of behavior in the group & .138 & .523 & .067 & .470 & .164 \\
\hline 24. Reflect on how my actions align with my values & .055 & .356 & .171 & .393 & .452 \\
\hline 25. Understand the priorities of others in the group & .386 & .383 & .094 & .356 & .175 \\
\hline $\begin{array}{l}\text { 26. Listen carefully to what is and isn't being said by group } \\
\text { members }\end{array}$ & .387 & .267 & .108 & .229 & .319 \\
\hline 27. Work with others toward a shared goal & .685 & .225 & .090 & -.002 & .225 \\
\hline 28. Work to resolve conflicts within the group & .677 & .146 & .322 & .000 & .112 \\
\hline 29. Align disparate viewpoints within the group & .411 & .343 & .454 & .078 & -.172 \\
\hline
\end{tabular}

Table 2.1.5 Rotated component score matrix evidencing 5 unique factors from college student leadership data 


\subsection{Classification using Discriminant Analysis - College Student Leadership Assessment}

Given no variable which actually measures the respondent's effectiveness as a leader, an attempt is made to utilize the data for "number of student organizations involved in" which ranges from 0 to 4 or more, and the binary variable "are you currently in a leadership role?" Fifty-four percent reported involvement in at least 3 organizations and nearly $86 \%$ said they were currently involved in a leadership role.

Consider respondents involved in three or more organizations, who are also in a leadership role. As a matter of convenience we call them max-leaders, and this group represents $49 \%$ of the sample.

Next, discriminant analysis is used to investigate the attributes that distinguish these highly active leaders from their less active counterparts. The discriminant analysis function is further validated by t-tests for significance of difference between the means of groups, which show that the max-leaders are significantly different from the rest of the dataset on several discriminating variables including taking the time to learn the informal traditions of the group, working toward a shared goal, and helping others enhance their skills and abilities (see Section 1.4).

Recall that $86 \%$ of the students said they were in a leadership role, and $89 \%$ are involved in at least 2 organizations. This commonality of involvement and leadership motivation portends a challenge in discerning the composition of the respondents' consciousness of context, self and others, without considering the high-involvement/activity respondents separately. Keep in mind that the measurement is not that of leadership effectiveness, but one that indicates a high level of involvement (three or more organizations), coupled with a current leadership role. This group of max-leaders, approximately half the dataset, will be compared to the less-involved second half to better understand which attributes best discriminate between the two groups.

Each construct is comprised of 8 variables, rated from 1 to 5, reflecting consciousness of context, self, and others, and has a maximum score of 40 . Three generally even levels are created for each consciousness construct. Low ratings are those $\leq 25$, mid-level ranges from 26 to 34 , and high scores are those ranging from 35-40. Given that virtually all respondents fell into the mid and high ranges, a greater focus is placed on understanding the composition of the mid and high levels, and the variables that can help discern the attributes of max-leaders in each of these groups versus others. First, 
however, the entire group of college student respondents is investigated to discern between the characteristics of the max-leaders versus others.

Results

Overall, discriminant analysis techniques, applied to the college student leadership dataset, effectively identify the variables which discriminate between the max-leaders and their less involved counterparts, but classification is not particularly fruitful. The same holds true as gender differences are investigated, where reasonable discrimination is evident, yet classification is weak. This is most likely the result of not having a good "outcome" variable such as a leadership effectiveness score. Several attempts were made to categorize respondents in meaningful ways that would lead to better classification, but again, without a useful outcome variable, such as effectiveness as determined externally, the classification results just border significance.

The stepwise discriminant analysis procedure (as described in Section 2.6 of the job-seeker analysis) was used to isolate the most discriminating variables. Box's M was insignificant, so pooled covariance matrices were used. To clarify focus on the discriminatory power of each relevant independent variable, the partial $F$ value is reported. Large $F$ values indicate stronger discriminatory power (Hair, et al, p. 269).

The structure matrix further validates the correlation of each predictor variable with the discriminant function, and also identifies the variables that are correlated with the discriminant function, yet may not have survived the stepwise analysis.

Effectiveness of the discriminant function is validated by Wilks' lambda which reveals how well each function separates cases into groups. It is the proportion of total variance in the discriminant score not explained by differences among groups. Smaller values mean better discriminatory ability.

\section{Discriminating Max-Leaders with Leadership Skills Variables}

It is fair to consider the most discriminatory variables as the behaviors that distinguish max-leaders from others. Thus, the stepwise discriminant analysis suggests that max-leaders work with others toward a shared goal $(\mathrm{F}=12.28)$, take time to understand the informal traditions of the group $(\mathrm{F}=9.69)$, and consider the needs of others in the group $(F=7.91)$. Table 2.2.1 provides details. 


\begin{tabular}{|c|c|c|c|c|c|c|c|}
\hline \multicolumn{8}{|c|}{ MAX-LEADERS STEPWISE DISCRIMINANT ANALYSIS Variables Entered/Removed } \\
\hline \multirow[t]{3}{*}{ Step } & \multirow[t]{3}{*}{ Entered } & \multicolumn{6}{|c|}{ Min. D Squared } \\
\hline & & \multirow[t]{2}{*}{ Stat } & \multirow{2}{*}{$\begin{array}{l}\text { Between } \\
\text { Groups }\end{array}$} & \multicolumn{4}{|c|}{ Exact $\mathrm{F}$} \\
\hline & & & & Statistic & df1 & df2 & Sig. \\
\hline 1 & $\begin{array}{l}\text { Work with others toward a } \\
\text { shared goal }\end{array}$ & .135 & 1.00 and 2.00 & 12.279 & 1 & 363 & .001 \\
\hline 2 & $\begin{array}{l}\text { Take time to understand } \\
\text { the informal traditions of } \\
\text { the group }\end{array}$ & .213 & 1.00 and 2.00 & 9.690 & 2 & 362 & $7.954 \mathrm{E}-5$ \\
\hline 3 & $\begin{array}{l}\text { Consider the needs of } \\
\text { others in the group }\end{array}$ & .262 & 1.00 and 2.00 & 7.909 & 3 & 361 & $4.016 \mathrm{E}-5$ \\
\hline
\end{tabular}

Table 2.2.1 Stepwise results for most discriminatory variables distinguishing max-leaders from others

The structure matrix further supports the importance of the primary independent variables by providing the correlation of these variables with the discriminant function. Specifically, working with others toward a shared goal has a loading of .72 and understanding informal traditions, .70. It is insightful to note that "considering the needs of others" is a predictor, yet does not hold correlational value with the discriminant function. One might infer that this is related to the fact that males and females differ significantly on "considering needs of others", with females significantly more likely to evidence this behavior in a leadership setting.

\section{Discriminating Gender with Leadership Skills Variables}

This leads again to the gender question, and whether discriminant analysis offers any insights as to how the genders might be discriminated given the leadership behavior variables measured. Stepwise discriminant analysis using pooled covariance matrices (Box's M was insignificant), reveals three key discriminators which are all reflective of the consciousness of SELF construct, two which women rate significantly higher, and one which males perceive as significantly more common behavior (Table 2.2.2)

The interesting result is that the variables that most distinguishes the genders are reflective of extant leadership theories. The first highly discriminating variable, "monitor how my emotions affect my interactions with others" ( $F=$ 7.80) is characteristic of the emotionally intelligent leader who monitors emotions and focuses on the more affective elements of leadership (Mayer, 
Salovey, \& Caruso, 2000). Recall from Section 1.4 that females rated their participation in this behavior significantly higher than males.

The second discriminating behavior, "follow through on my commitments" ( $F$ $=7.02$ ) was also rated significantly higher by females and might be considered to affirm path-goal theory where leaders are achievement-oriented (Northouse, p. 130).

The final discriminator, "capitalize on my strengths" $(F=6.86)$, was rated significantly higher by males, and is reflective of the skills approach to leadership which emphasizes learning and development of skills, suggesting that knowledge and abilities are needed for effective leadership (Northhouse, p. 39).

Examination of the structure matrix supports the three discriminatory variables with loadings of -.569 for capitalizing on strengths, .533 for monitoring emotions, and .386 for following through on commitments (recall the threshold of .40). Wilks' lambda is not particularly encouraging as reflected in the classification results, with $\lambda=945$. However $\chi=20.49$, significant at $p$ $<.000$, clearly supports the hypothesis that the means of the functions are equal across groups.

\begin{tabular}{|c|c|c|c|c|c|c|c|}
\hline \multicolumn{8}{|c|}{ GENDER - Discriminating Variables Entered/Removed } \\
\hline \multirow[t]{3}{*}{ Step } & \multirow[t]{3}{*}{ Entered } & \multicolumn{6}{|c|}{ Min. D Squared } \\
\hline & & \multirow[t]{2}{*}{ Stat } & \multirow{2}{*}{$\begin{array}{l}\text { Between } \\
\text { Groups }\end{array}$} & \multicolumn{4}{|c|}{ Exact $F$} \\
\hline & & & & Stat & df1 & $\mathrm{df} 2$ & Sig. \\
\hline 1 & $\begin{array}{l}\text { Capitalize on my } \\
\text { strengths }\end{array}$ & .089 & $\begin{array}{l}\text { Male and } \\
\text { Female }\end{array}$ & 6.863 & 1 & 363.000 & .009 \\
\hline 2 & $\begin{array}{l}\text { Monitor how my } \\
\text { emotions affect my } \\
\text { interactions with } \\
\text { others }\end{array}$ & .203 & $\begin{array}{l}\text { Male and } \\
\text { Female }\end{array}$ & 7.804 & 2 & 362.000 & .000 \\
\hline 3 & $\begin{array}{l}\text { Follow through on } \\
\text { my commitments }\end{array}$ & .274 & $\begin{array}{l}\text { Male and } \\
\text { Female }\end{array}$ & 7.020 & 3 & 361.000 & .000 \\
\hline
\end{tabular}

Table 2.2.2 Stepwise discriminant analysis distinguishing gender

Given Max-Leaders, Discriminate Gender

Consider only the max-leaders. Using discriminant analysis, stepwise method, with separate covariance matrices since Box's $M$ is significant in this case, two variables surface which discriminate the genders, given that they are max- 
leaders involved in more than 3 student organizations, and in a leadership role. The most discriminating variable, considering the needs of others in the group $(F=11.01)$, was rated significantly higher by females (Table 2.2.3). Interestingly, this behavior is characteristic of a transformational leader who emphasizes intrinsic motivation, and is concerned with assessing followers' motives and satisfying their needs (Northouse, p. 175). Results from the structure matrix support the usefulness of the variable which has a loading score of -475 .

\begin{tabular}{|c|c|c|c|c|c|c|c|}
\hline \multicolumn{8}{|c|}{ Discriminating Gender of MAX-LEADERS, Variables Entered/Removed } \\
\hline \multirow[t]{3}{*}{ Step } & \multirow[t]{3}{*}{ Entered } & \multicolumn{6}{|c|}{ Min. D Squared } \\
\hline & & \multirow[t]{2}{*}{ Stat } & \multirow{2}{*}{$\begin{array}{l}\text { Between } \\
\text { Groups }\end{array}$} & \multicolumn{4}{|c|}{ Exact $\mathrm{F}$} \\
\hline & & & & Statistic & df1 & df2 & Sig. \\
\hline 1 & $\begin{array}{l}\text { Improve my } \\
\text { abilities }\end{array}$ & .221 & $\begin{array}{l}\text { Male and } \\
\text { Female }\end{array}$ & 7.897 & 1 & 180 & .005 \\
\hline 2 & $\begin{array}{l}\text { Consider the } \\
\text { needs of } \\
\text { others in the } \\
\text { group }\end{array}$ & 618 & $\begin{array}{l}\text { Male and } \\
\text { Female }\end{array}$ & 11.007 & 2 & 179 & $3.101 \mathrm{E}-5$ \\
\hline
\end{tabular}

Table 2.2.3 Stepwise discriminant analysis distinguishing gender among max-leaders

The second discriminating variable, improving one's abilities, $(F=7.89$, structure matrix loading $=.597)$ did not evidence significant mean differences between the genders in the entire dataset, nor among the max-leaders. This suggests that discriminant analysis permits the identification of important variables that distinguish the genders yet may not be obvious from difference analysis such as in t-tests for significant differences between means. Thus, discriminant analysis is a necessary step, particularly in behavioral research, toward a better understanding of underlying differences between groups or categories of respondents.

Evaluating the ability of the discriminant function to separate cases into gender groups, $\lambda=89$, and classification results indicate better than chance accuracy with $72.4 \%$ of the estimation set classified correctly and $63 \%$ of the hold-out sample correct. Both exceed the better-than-chance criterion of $61.25 \%$.

\section{Discriminating Max-Leaders Given Gender}

Consider only males, and which variables might discriminate max-leaders from others of the same gender. Results from the discriminant analysis suggest 
that it is simply working to build a sense of team $(F=8.18)$ that distinguishes the male max-leader from his less involved and leading counterparts (Table 2.2.4). Team leadership is typical of the organizational setting, where the leader processes complex information for action taking (Northouse, p.224). No significant differences between genders are evident with regard to team building. Again, discriminant analysis has provided information not clarified through analysis of mean differences.

\begin{tabular}{|c|c|c|c|c|c|c|c|}
\hline \multicolumn{8}{|c|}{ MALES ONLY - Discriminating Max-Leaders - Variables Entered/Removed } \\
\hline \multirow[t]{3}{*}{ Step } & \multirow{3}{*}{ Entered } & \multicolumn{6}{|c|}{ Min. D Squared } \\
\hline & & \multirow[t]{2}{*}{ Stat } & \multirow{2}{*}{$\begin{array}{l}\text { Between } \\
\text { Groups }\end{array}$} & \multicolumn{4}{|c|}{ Exact $F$} \\
\hline & & & & Statistic & df1 & df2 & Sig. \\
\hline 1 & $\begin{array}{l}\text { Work to build a } \\
\text { sense of team }\end{array}$ & .295 & $\begin{array}{l}1.00 \text { and } \\
2.00\end{array}$ & 8.182 & 1 & 109.000 & .005 \\
\hline
\end{tabular}

Table 2.2.4 Male category, discriminating max-leaders from base

On the other hand, female max-leaders focus on identifying external influences on the group $(F=4.05), p<.05$ (Table 2.2.5). The behavior may be considered reflective of a situational approach where the leader adapts his/her leadership style to the situation, perhaps as a result of external influences. Additionally, contingency theory states that effective leadership is contingent on matching a leader's style to the right setting. Identifying external influences serves to evaluate the situation, which the female max-leader leader then adapts to the setting.

\begin{tabular}{|c|c|c|c|c|c|c|c|}
\hline \multicolumn{7}{|c|}{ FEMALES ONLY - Discriminating Max-Leaders - Variables Entered/Removed } \\
\hline \multirow{2}{*}{ Step } & \multirow{2}{*}{ Entered } & \multicolumn{5}{|c|}{ Min. D Squared } \\
\cline { 3 - 8 } & & Stat & $\begin{array}{c}\text { Between } \\
\text { Groups }\end{array}$ & \multicolumn{4}{|c|}{ Exact F } \\
\cline { 4 - 8 } & & & Statistic & df1 & df2 & Sig. \\
\hline 1 & $\begin{array}{l}\text { Identify external } \\
\text { influences on group }\end{array}$ & .061 & $\begin{array}{l}1.00 \text { and } \\
2.00\end{array}$ & 4.053 & 1 & 262.000 & .045 \\
\hline
\end{tabular}

Table 2.2.5 Female category, discriminating max-leaders from base

These methods of creating variables to enable discriminant analysis do offer interesting insights, but without a specific categorical dependent variable, the application of discriminant analysis is limited. Thus in the following and final section, cluster analysis is investigated as a more appropriate tool for classification and analysis of the college student leadership data. 


\subsection{Cluster Analysis to Segment Students on Leadership Behaviors}

This section investigates the application of clustering techniques to the college student leadership behavior data, then profiles and validates the resulting clusters. See Section 2.5 of the Job Seeker analysis for additional details on the methods and options for using cluster analysis.

Cluster analysis allows the researcher to take a different look at the data, with no preconceived notions regarding profiles, similarities, or performance measures. This analysis simply aims to segment the college student leadership data into meaningful clusters. Then these clusters are reviewed, evaluated and discussed to better understand the behaviors that link those within a cluster, and differentiate them from those in other clusters.

The final clustering method used here is a two-step cluster program which has the ability to quickly determine the appropriate number of clusters, then classify them using a nonhierarchical routine. The procedure is relatively new, but as recommended by Hair et. al. (p. 497), it does prove to be useful in this application due to the sample size, over 500 cases, and the number of variables be analyzed (the 24 leadership behaviors). Garson (2009) further encourages the use of the two-step method for large datasets using both continuous data and categorical variables with three or more levels.

The two steps are a pre-clustering step where cases are divided into small subclusters, followed by a second clustering of the sub-clusters into the desired or pre-defined number of clusters. An automatic selection of clusters is optional, but results in only two clusters given the leadership dataset, which does not yield the interpretability being sought. Thus, the appropriate number of clusters must be determined which will be discussed further below.

The two-step procedure is based on Banfield and Rafferty's (1993) work with clustering methods for continuous variables based on the reduction in loglikelihood when two clusters are merged. Further, the two-step procedure extends the work of Melia and Heckerman (1998) who took a similar probabilistic approach to clustering categorical variables. Zhang et al, 1996 developed BIRCH clustering for larger datasets, reducing them to sub-clusters which are analyzed in a second step much like traditional clustering methods. The two-step procedure innovatively combines these works, resulting in an effective clustering solution for the leadership dataset due to its size and the number and types of variables being investigated.

The pre-clustering stage uses a cluster-feature tree (CF tree) with nodes leading to leaf nodes, the result of a sequential clustering method described by Theodoridis and Koutroumbas (1999). Cases begin at the base, or roots of the 
tree, and climb toward nodes and up to leaf nodes with the closest match. If the distance measure for the case is above the threshold level, thus no adequate match is found, the case begins a new leaf node. If the CF tree fills and cannot accept new leaf entries in a node, it is split, using the most-distant pair in the node as seeds. Should this recursive process build the tree beyond maximum size, the threshold distance is increased and the tree is rebuilt, allowing new cases, and continuing the process until all cases are read. For the leadership dataset, the clustering specified includes maximum of three levels of nodes for the $\mathrm{CF}$ tree, with eight entries per node maximum.

The log-likelihood distance measure is used because it can handle both continuous (normal distribution assumed) and categorical variables (multinomial distribution assumed). The distance between two clusters is based on the decrease in log-likelihood as they are combined into one cluster. With the log-likelihood distance for two-step clustering algorithm, the distance between clusters is defined as

$$
d(i, j,)=\xi+\xi,-\xi_{, j>}
$$

where

$$
\xi=-N_{v}\left(\sum_{k=1}^{K^{A}} 1 \log \left(\sigma+\sigma_{\ldots}\right)+\sum_{k=1}^{K^{B}} \hat{E}_{v k}\right)
$$

and

$$
\hat{E}_{v k}=-\sum_{l=1}^{r}{ }_{i v}^{\cdots k l} \log \frac{N_{v k l}}{N_{v}} .
$$

$K^{A} \quad$ Total number of continuous variables used in the procedure.

$K^{B} \quad$ Total number of categorical variables used in the procedure.

$R_{k} \quad$ The range of the $k^{\text {th }}$ continuous variable.

$N \quad$ Number of data records in total.

$N_{k} \quad$ Number of data records in cluster $k$. $\hat{\mu} \quad$ The estimated mean of the $k^{\text {th }}$ continuous variable across the entire

$\sigma \quad$ Estimated variance of the $k^{\text {th }}$ continuous variable across the entire dataset.

$\hat{\mu}_{\text {. }} \quad$ Estimated mean of the $k^{\text {th }}$ continuous variable in cluster $j$.

$\sigma_{, 2} \quad$ Estimated variance of the $k^{\text {th }}$ continuous variable in cluster $j$. 
$d(j, s) \quad$ Distance between clusters $j$ and $s$.

$<j, s>$ Index that represents the cluster formed by combining clusters $j$ and $s$.

\section{Variable Importance}

The relative contribution of each variable to the cluster can be computed for both categorical and continuous variables. For categorical variables, the importance measure is chi-square distributed and for continuous variable the measure is based on Student's t-test. Computations for both are given here, but the application to the college student leadership data will discuss the significance of the probability measures which are more straightforward graphically.

Categorical variables are based on the importance measure:

$$
\chi=\sum_{l=}^{L_{k}}\left(\frac{N_{j k l}-N_{k l}}{N_{k l}}\right)^{-}
$$

Where

$N_{j k l}$ is the number of data records in cluster $j$ whose $k^{\text {th }}$ categorical variable takes the $l^{\text {th }}$ category.

$N_{k l}$ is the number of data records in the $k^{\text {th }}$ categorical variable that take the $l^{\text {th }}$ category.

$L_{k}$ is the number of categories for the $k^{\text {th }}$ categorical variable.

Under the null hypothesis the above measure is distributed as $\chi$ with $L_{k}$ degrees of freedom. Again, the importance measure can be reported as $\chi$ or its significance.

Continuous variables are based on the importance measure:

$$
t=\frac{\mu-\iota_{0}}{\sigma_{,:} / \sqrt{N_{k}}} .
$$

Under the null hypothesis, this is distributed as $t$ with $N_{k}-1$ degrees of freedom. Significance level is two-tailed, and again, either the statistic or its significance level can be reported as the importance measure. 


\section{Determining Number of Clusters}

The procedure uses an algorithm based in part on Bayesian (BIC) or Akaike (AIC) information criteria loss to determine the optimal number of clusters. As mentioned earlier, this automatic determination results in only two clusters which is not preferred given the leadership application. The preference is to identify clusters comprised of cases that behave similarly. The objective is to profile and understand the behaviors within the cluster, and differences between the clusters, so as to suggest improvements that have the potential to transition members to a cluster evidencing stronger leadership tendencies. The automatic cluster number option was overridden and a random sample of 100 cases taken through several variations of hierarchical clustering procedures to determine the appropriate number of clusters as suggested by Garson (2009).

To determine the appropriate number of clusters, the agglomeration schedule was reviewed, looking for substantial changes in heterogeneity, meaning how different observations in one cluster are from those in another (Hair et al, p.526). The agglomeration coefficient measures the increase in heterogeneity occurring from the combination of two clusters. Hair et al. suggest a reasonable approach to determining the number of clusters is to measure the percentage change in heterogeneity. "When large increases occur in moving from one stage to the next, the researcher selects the prior cluster solution because the new combination is joining quite different clusters." (Hair, et al, p. 527). A $27 \%$ change in the agglomeration coefficient is evident between three and four clusters using a complete linkage method, thus the three cluster solution was selected.

Several steps were initially taken which further validate the use of a three cluster solution. A random sampling of 100 cases were tested using only the three construct variables (SELF, CONTEXT, OTHERS). Complete linkage and Ward's method resulted in four cluster solutions, while single linkage returned three clusters. Next, all cases and all 24 leadership variables were tested under the Ward's linkage method which resulted in a three cluster solution with a $4.4 \%$ change between three and four clusters. As mentioned above, results from the complete linkage agglomeration schedule supported a three cluster solution.

\section{Cluster Profiles}

As discussed earlier, the two-step clustering method was used on the college student leadership data because of its ability to handle both continuous and categorical data, as well as its flexibility with larger sample sizes. The cluster 
analysis yields three uniquely profiled groups, with membership distributed in a reasonable manner with $22 \%$ in cluster $1,54.3 \%$ in cluster two and $23.7 \%$ in cluster 3 . The cluster sizes are reasonable in that one might expect around 20$25 \%$ of college student respondents to fall in the lowest and highest cluster categories based on self-assessments of leadership skills, the balance falling in the middle category.

Based on the profiles to be discussed, the clusters are nicknamed as follows: Cluster 1, less involved, less others-oriented, cluster 2 are the self-improvers, and cluster 3 are the more involved leaders with some distinctive characteristics.

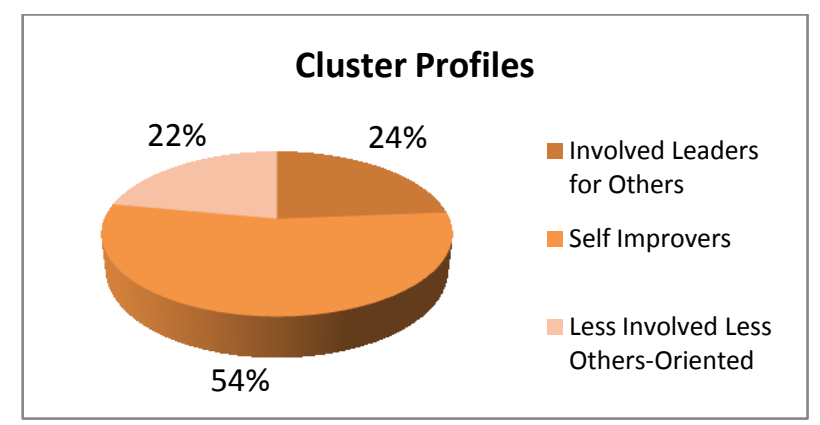

Figure 2.3.1 Cluster Profiles

\section{Cluster 1 - Less Involved, Less Others-Oriented}

Variable importance plots are used to graphically display the variables' relative contribution to the cluster. The first cluster is profiled as "less involved, less others oriented." These are essentially the lowest self-scoring students. Figure 2.3.2 suggests significant chi-square values for the categorical variables in the analysis, level of involvement, year in school and gender. The most differentiating impact from categorical variables is from involvement level. This group holds nearly $85 \%$ of the students who were not involved in any student organizations. Members tend to be seniors and graduate students which may explain a lower level of involvement in on-campus student organizations. 


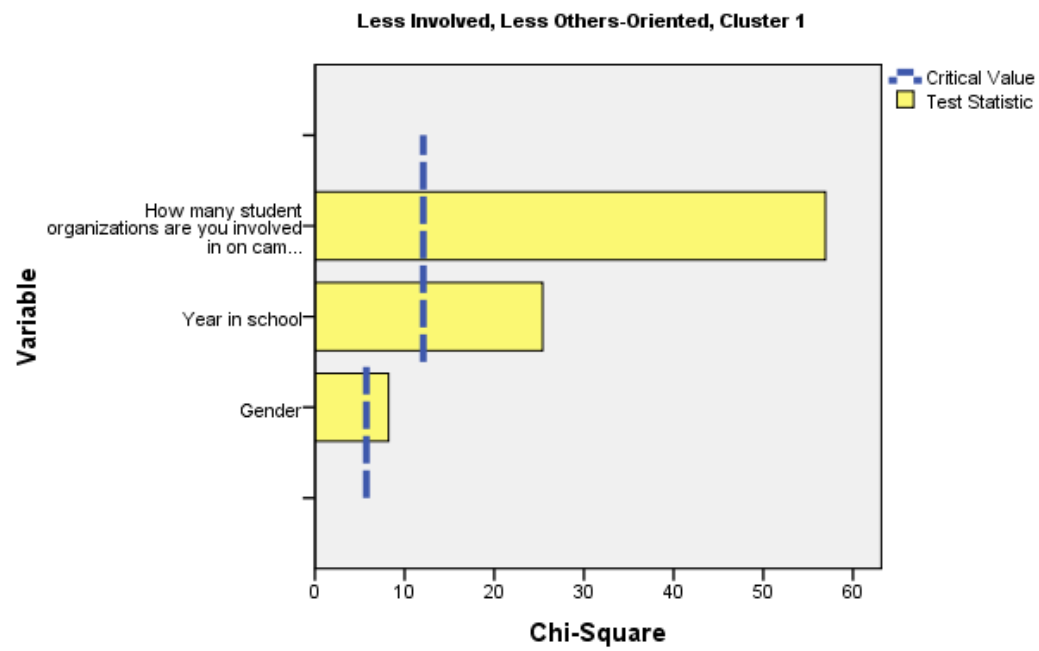

Figure 2.3.2 Cluster 1 - Categorical discriminators for less involved, less others-oriented group

The top five most important variables contributing to cluster membership will be discussed. While others do contribute significantly, five is a manageable number to recall for reference and discuss here.

The top two are both variables related to consciousness of OTHERS; thinking about how decisions are received by others in the group, as well as being concerned about resolving conflicts within the group. The next most important variables affecting cluster membership are two CONTEXT variables which are thinking about how the environment influences their leadership style, and tailoring their style to the situation. Willingness to help others enhance their skills was the fifth most important variable in contributing to group membership. These are the five variables with the most significant probability scores with regard to each variable's contribution to group membership in the less involved, less others-oriented cluster (Table 2.3.1).

\begin{tabular}{|lcc|}
$\begin{array}{l}\text { Less Involved, Less Others- } \\
\text { Oriented Cluster } \\
\text { Variable }\end{array}$ & t-statistic & p-value \\
\hline Think about Decisions Received & -9.6 & .000 \\
\hline Resolve Conflicts & -9.2 & .000 \\
\hline Environment Influences Style & -9.1 & .000 \\
\hline Tailor Style to Situation & -9.0 & .000 \\
\hline Help Others Enhance Skills & -8.9 & .000 \\
\hline
\end{tabular}

Table 2.3.1 Variables most significantly contributing to membership in "Lessinvolved, less others-oriented" cluster 1. 
Again, as this is the lowest-scoring group, a development focus on improving in these areas could potentially move this group to a higher level of leadership.

\section{Cluster 2 - Self Improvers}

Given that three clusters were specified, this middle group had no variables that significantly contributed to group membership. Working on one's limitations approached significance, thus the cluster is nicknamed "selfimprovers". None of the categorical or continuous variables serve as significant discriminators of group membership, but the variable importance plots offer a directional view into the respective importance of each variable within the cluster. This group may be more self-oriented, working on their limitations, improving abilities, capitalizing on personal strengths, and following through, all variables of the SELF construct.. Note that the second most important variable (although insignificant) is thinking about how one's leadership style aligns with group culture, a consciousness of CONTEXT variable, suggesting a willingness to learn leadership skills. Recall that this second cluster of self-improvers rates significantly lower on their mean scores for all these variables compared to their cluster 3 counterparts.

\section{Cluster 3 - Involved Leaders for Others}

Members of the third cluster, dubbed "Involved Leaders for Others" are significantly differentiated by their high level of involvement in student organizations on campus (Figure 2.3.3).

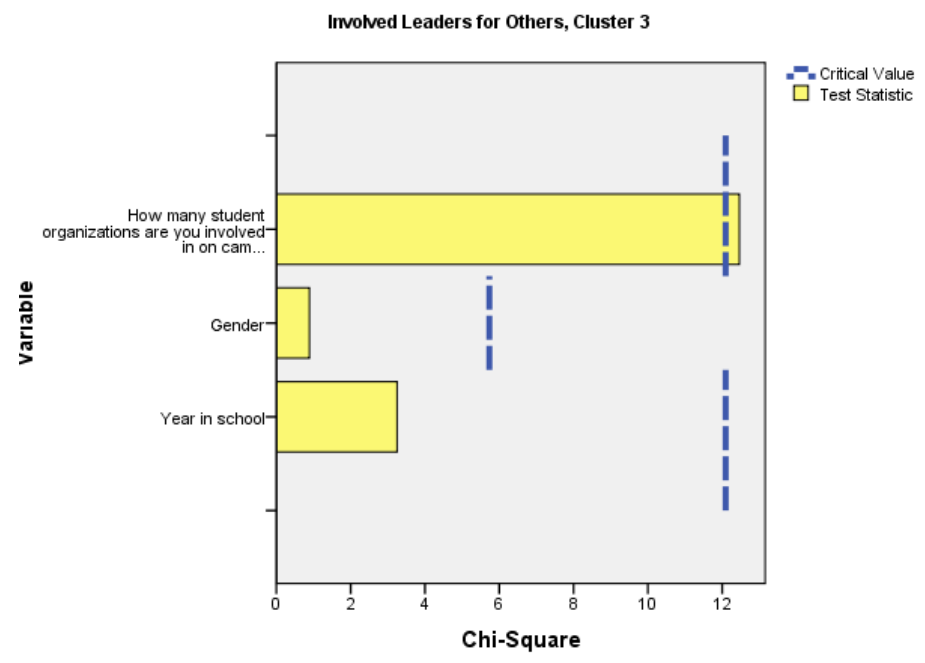

Figure 2.3.3 Level of involvement differentiates "Involved Leaders for Others" - Cluster 3 
This top-ranking group is distinguished by their others-centeredness including working to resolve conflicts within the group, team-building, and considering the needs of others in the group, all variables from the OTHERS consciousness construct. They next look internally and think about how they might improve their abilities and establish a shared goal (SELF variables). Table 2.3.2 reviews Student's t for each variable and its significance.

\begin{tabular}{|lcc|}
\hline $\begin{array}{l}\text { Involved Leaders for Others } \\
\text { Cluster } \\
\text { Variable }\end{array}$ & $\boldsymbol{t}$-statistic & p-value \\
\hline Resolve Conflicts & 16.9 & .000 \\
\hline Work to Build Team & 16.3 & .000 \\
\hline Consider Needs of Others & 15.8 & .000 \\
\hline Improve my Abilities & 15.6 & .000 \\
\hline Work Toward a Shared Goal & 14.8 & .000 \\
\hline
\end{tabular}

Table 2.3.2 Variables most significantly contributing to membership in Involved Leaders for Others - cluster 3

\section{Validating the Cluster Solution with Discriminant Analysis}

Cluster membership can be used as the grouping variable in discriminant analysis as a means for validating the final cluster solution (Punj, 1983, Garson, 2009;). Punj suggests that after developing the cluster solution on one sample, discriminant functions are derived and applied to a second (hold out) sample. Figure 2.3.4 shows the distribution of the discriminant scores for the first discriminant function applied to each of the three clusters derived in the two-step cluster analysis. The distribution of discriminant scores for each cluster is substantially separate.

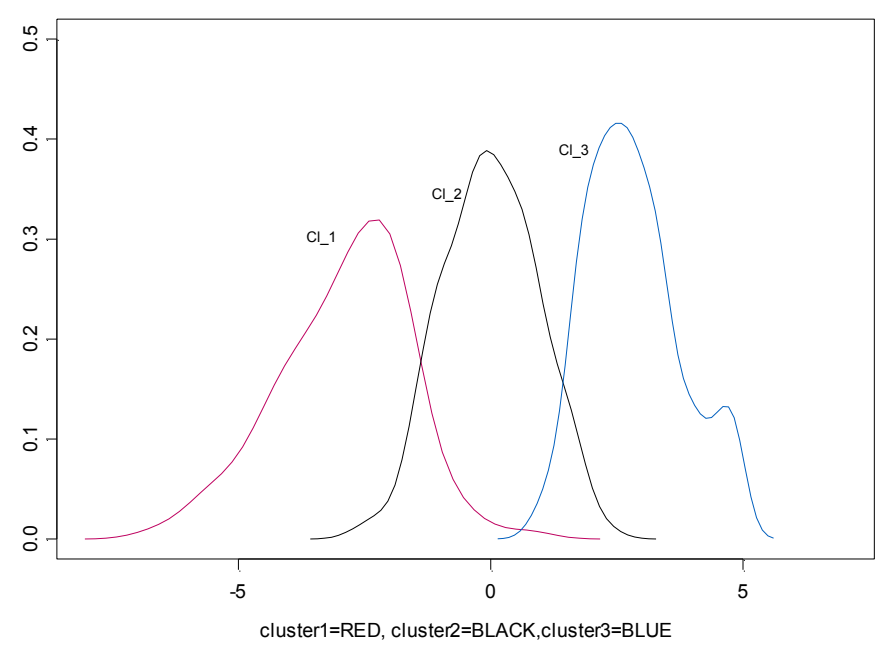

Figure 2.3.4 Discriminant scores for each cluster from first discriminant function 
To assess model fit, consider Wilks' $\lambda=.186$ for the first discriminant function, which suggests that the model separates cases into groups very effectively with the proportion of total variance in the discriminant scores not explained by differences among the groups at only $18.6 \%$. Recall, smaller values of lambda suggest greater discriminatory power of the function.

The stepwise procedure was used, where at each step the variable that maximizes the Mahalanobis distance between the two closest groups is entered into the solution. Interestingly, "work to build a sense of team" is the first variable entering the procedure, followed by considering the needs of others in the group. These two variables, elements of the consciousness of OTHERS construct, serve to differentiate clusters 1 and 2. This supports the profiling of cluster 1 members who tended to be significantly less conscious of others given the attribute importance ratings supplied by the two-step cluster procedure.

Substantially separating cluster 2 (Self-Improvers) and cluster 3 (Involved Leaders for Others) are propensity to improve one's abilities and monitoring how emotions affect interactions with others, both SELF construct variables. A simple tally of the variables and the clusters they differentiate reveals that the less involved, less others-oriented members of cluster 1 were differentiated from cluster 2, Self-Improvers, primarily on OTHERS and CONTEXT variables. It is primarily CONTEXT and SELF variables that distinguish SelfImprovers (cluster 2) from the Involved Leaders for Others in cluster 3. Further, extent of organizational involvement on campus further discriminates between Self-Improvers and Involved Leaders. These findings clearly support the suggestion that if the less involved, less others-oriented members in cluster 1 were to focus on being more conscious of others and the context of the leadership situation they could potentially transition to a higher level in cluster 2. They could potentially improve these skills through greater involvement in campus organizations. Such involvement, plus additional focus on the SELF attributes has the potential to transition them all the way to cluster 3, Involved Leaders for Others.

The second function offers some discrimination, but just borders significance with $\lambda=.937$, significant at .066 , somewhat outside a useful threshold. Figure 2.3.5 provides graphic support for the second discriminant function. 


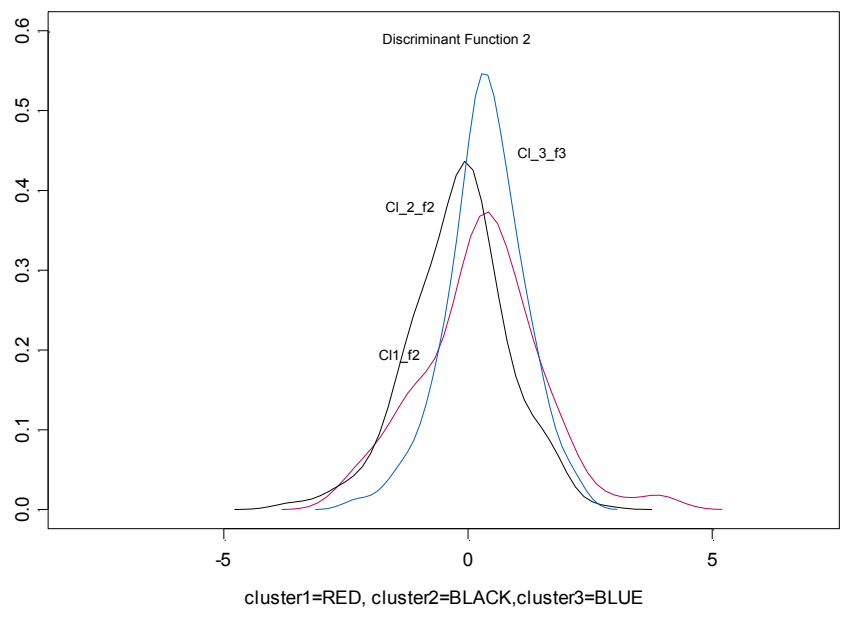

Figure 2.3.5 Second discriminant function

Looking to the classification table (Table 2.3.3) for practical results, it is found that $92 \%$ of the cases from which the function was built are classified correctly, and $87 \%$ of the holdout sample is correctly classified. Specifically, cluster 1, whose members are less involved, less-others-oriented, are classified correctly $83.9 \%$ of the time, and cluster 2 Self-Improvers $84.5 \%$ of the time. Also, $94.6 \%$ of the hold-out sample cases of the involved, more othersoriented, leaders, cluster 3 , were correctly classified. Overall the discriminant analysis approach to validating the clusters proves worthwhile, and suggests the clusters are stable. 


\begin{tabular}{|c|c|c|c|c|c|c|c|}
\hline \multicolumn{8}{|c|}{ Classification Results ${ }^{a, b}$} \\
\hline & & & \multirow{2}{*}{$\begin{array}{l}\text { with gender, yrsch, } \\
\text { orgs }\end{array}$} & \multicolumn{4}{|c|}{ Predicted Group Membership } \\
\hline & & & & Cluster 1 & Cluster 2 & Cluster 3 & Total \\
\hline \multirow[t]{8}{*}{ Cases Selected } & \multirow[t]{8}{*}{ Original } & \multirow[t]{4}{*}{ Count } & 1 & 78 & 3 & 0 & 81 \\
\hline & & & 2 & 9 & 168 & 14 & 191 \\
\hline & & & 3 & 0 & 2 & 82 & 84 \\
\hline & & & Ungrouped cases & 5 & 3 & 3 & 11 \\
\hline & & \multirow[t]{4}{*}{$\%$} & 1 & 96.3 & 3.7 & .0 & 100.0 \\
\hline & & & 2 & 4.7 & 88.0 & 7.3 & 100.0 \\
\hline & & & 3 & .0 & 2.4 & 97.6 & 100.0 \\
\hline & & & Ungrouped cases & 45.5 & 27.3 & 27.3 & 100.0 \\
\hline \multirow[t]{8}{*}{ Cases Not Selected } & \multirow[t]{8}{*}{ Original } & \multirow[t]{4}{*}{ Count } & 1 & 26 & 5 & 0 & 31 \\
\hline & & & 2 & 8 & 73 & 5 & 86 \\
\hline & & & 3 & 0 & 2 & 35 & 37 \\
\hline & & & Ungrouped cases & 3 & 6 & 3 & 12 \\
\hline & & \multirow[t]{4}{*}{$\%$} & 1 & 83.9 & 16.1 & .0 & 100.0 \\
\hline & & & 2 & 9.3 & 84.9 & 5.8 & 100.0 \\
\hline & & & 3 & .0 & 5.4 & 94.6 & 100.0 \\
\hline & & & Ungrouped cases & 25.0 & 50.0 & 25.0 & 100.0 \\
\hline \multicolumn{8}{|c|}{ a. $\mathbf{9 2 . 1} \%$ of selected original grouped cases correctly classified. } \\
\hline b. $\mathbf{8 7 . 0} \%$ of unselecte & original $\mathrm{g}$ & uped ca & es correctly classifiec & & & & \\
\hline
\end{tabular}

Table 2.3.3 Discriminant analysis classification results using cluster membership as grouping variable 


\subsection{Summary and Future Research}

The management issue was to validate a self-assessment tool employed by researchers studying college student leadership behaviors. The methods used were factor analysis to assess reliability of the assessment tool, discriminant analysis to understand the variables separating higher performing leaders from others, and cluster analysis to ascertain the natural clustering of behavioral variables related to leadership. Three underlying constructs of the leadership experience were identified and validated. These include consciousness of context, self and others.

Another objective was to use the data to suggest areas for improvement in the consciousness constructs (context, self and others) which reflect the behaviors of higher level leaders (at least from the perspective of self-perceptions). Starting from oneself, it was recommended that the initial focus be on improving one's abilities, aligning one's actions with values, then working on one's limitations. Considering consciousness of others, the immediate focus that most differentiated low from mid-level leaders was thinking about how one's decisions are received by the group. To generate improvement from there, a focus on helping others enhance their skills and abilities was suggested. Also, context must be considered. The data suggests that those scoring lowest on consciousness of context are primarily different from their mid-level counterparts in their thinking about how they align their leadership style with group culture. The next step suggested was to understand how the group's environment influences one's leadership style, then tailoring the style to the situation. These elements most differentiated college students in their self-perceived leadership behaviors. The differences evident in the behavioral data were effectively interpreted to make suggestions for improvements.

Cluster analysis enabled the division of respondents into meaningful clusters based on specific, self-reported behaviors. In leadership settings, those who are more involved were also more others-oriented. They prioritize resolving any conflicts within the group, then they work on team-building. Considering the needs of others plays a key role as does improving one's abilities. Working toward a shared goal further contributed to a student being categorized into this leadership group.

The investigation of gender differences was fruitful. Findings from research question one suggested that women and men college students lead differently. Women demonstrated significantly higher levels of emotionally intelligent leadership in the constructs of consciousness of self and consciousness of others. This supports findings that women tend to demonstrate higher 
competence in individual and group values of the social change model of leadership than men (Dugan et al., 2008). It also supports the idea that women have more democratic and relational leadership styles than men (Eagly \& Carli, 2007).

In examining the individual measures from research question one, women more specifically tend to put the needs of others in front of their own, listen to others, and think about how their decision will be received more often than men. This concern for others also reflects democratic leadership, which emphasizes collaboration, shared power, and group decision-making (Northouse, 2007). Women's greater capacities for the consciousness of self measures of following through on their commitments and aligning their actions with their values reflect findings by Dugan et al. (2008) and Haber and Komives (2009) that women demonstrate higher levels of commitment and congruence from the social change model of leadership than men.

The behavior of considering needs of others was particularly prominent, and among the students with the highest scores of all three levels of consciousness, women still held significantly higher scores than men on this measure. Considering the needs of others seems to be the behavior that most differentiates the genders at the highest self-perceived levels of leadership. This finding along with the overall pattern of findings from this study suggest that women have higher levels of many of the consciousness of others measures than men, which is consistent with the literature on gender differences in transformational leadership (Bass \& Riggio, 2006; Eagly \& Carli, 2007) and the group values of socially responsible leadership (Dugan et al., 2008; Haber, 2006).

Men in the study demonstrated higher levels of the consciousness of self measure of capitalizing on strengths than women. While this measure does not specifically have to do with self-esteem, it does reflect acknowledgement of strengths and self-perception of ability to use strengths. This could reflect past research that men tend to demonstrate greater confidence and selfefficacy in leadership than women (Dugan \& Komives, 2007; Kezar \& Moriarty, 2000). Additionally, highly involved men also worked on their limitations more often than women. This goes hand in hand with trying to build strengths and capitalizing on them.

In examining gender differences based on levels of involvement, it does not appear that levels of involvement by gender provide many significant findings. For men and women involved moderately (two or three organizations), women scored significantly higher than men in the constructs of consciousness of self (for two organizations) and consciousness of others (for three organizations). 
There were no overall differences in levels of consciousness for respondents with low involvement (one organization) or high involvement (four or more organizations). It appears as if moderate involvement is associated with gender differences in measures of emotionally intelligent leadership. This emergence of gender differences for moderate involvement but not for high or low involvement warrants additional exploration as to the role that gender plays based on different levels and types of involvement in developing students' emotionally intelligent leadership.

Although there were no differences by gender in the three constructs of consciousness for those students involved in four or more organizations, gender differences did emerge for the individual measures. Unlike other findings throughout this study, men tended to work on their limitations, capitalize on their strengths and align different points of view more than women. In examining the shift in gender differences from the moderately involved students to the highly involved students, there may be something to be said about women being overburdened or overwhelmed with higher levels involvement or men benefiting more from higher levels of involvement. These possible phenomena would be worth exploring to understand what may contribute to these differences along with or beyond the sheer number of organizations in which one is involved. Further exploration into how men and women's emotionality intelligent leadership measures shift depending on levels of involvement would also provide additional insight into the role of involvement in college men and women's emotionally intelligent leadership outcomes.

It is important to note that throughout both research questions there were no significant differences for the construct consciousness of context. A significant difference emerged for one of the consciousness of context measures, whereby women involved in two organizations recognize patterns or behaviors in a group more often than men. Beyond this narrow finding, it appears as if men and women have similar levels of awareness of the larger environment in which they operate. Overall, women tend to demonstrate higher levels of the consciousness of self and consciousness of others capacities of emotionally intelligent leadership than men. 


\section{References Classification and Analysis of College Student Leadership}

Arvey, R., Rotundo, M., Johnson, W., Zhang, Z. \& McGue, M., (2006). The determinants of leadership role occupancy: Genetic and personality factors. Leadership Quarterly, 17, 1-20.

Arvey, R., Zhang, Z, Krueger, D. \& Avolio, B. (2007). Development and genetic determinants of leadership role occupancy among females. Journal of Applied Psychology, 92 (3), 693-706.

Astin, A. W. (1993). What matters in college?: Four critical years revisited. San Francisco: Jossey-Bass.

Avolio, B. \& Luthans F. (2006). The High Impact Leader. New York: McGraw Hill.

Avolio, B. J. \& Gardner, W. L. (2005). Authentic leadership development: Getting to the root of positive forms of leadership. The Leadership Quarterly, 16, 315-338.

Banfield, J. D., and Rafferty, A. E., (1993), Model based Gaussian and nonGaussian clustering, Biometrics 49, 803-821.

Bar-On, R. (2006). The Bar-On model of emotional-social intelligence (ESI). Psicothema, 18, supl., 13-25.

Bar-On, R. (1997). The Bar-On emotional quotient inventory (EQ-i): A test of emotional intelligence. Toronto, Canada: Multi-Health Systems.

Bass, B. (1985). Leadership and performance beyond expectations. New York: The Free Press.

Bass, B. M. \& Riggio, R. E. (2006). Transformational leadership (2nd ed.). Mahwah, NJ: Lawrence Erlbaum Associates.

Blake R. \& Mouton, J. (1978). The new managerial grid. Houston, TX; Gulf Publishing Company.

Blanchard, K. H., Zigarmi, D. \& Nelson, R. B. (1993). Situational leadership after 25 years: a retrospective. The Journal of Leadership Studies, 1(1), 21-36. 
Boyatzis, R. (1982). The competent manager: A model for effective performance. New York: John Wiley and Sons.

Brackett, M. A., Rivers, S. E. \& Shiffman, S. (2006). Relating emotional abilities to social functioning: A comparison of self-report and performance measures of emotional intelligence. Journal of Personality and Social Psychology, 91(4), 780-795.

Capacity. (2009). Dictionary.com. Houghton Mifflin Company. Retrieved June 30, 2009 from http://dictionary.reference.com/browse/capacity

Caruso, D. (2003, November). Defining the inkblot called Emotional Intelligence. Issues and Recent Developments in Emotional Intelligence,1(2), Retrieved 09 September 2009, from http://www.eiconsortium.org

Chaleff, I. (2003). The courageous follower: Standing up to and for our leaders. San Francisco, CA: Barrett-Koehler Publishers, Inc.

Cherniss, C. (1999). The business case for emotional intelligence in organizations. Retrieved 09 September 2009 from http://www.eiconsortium.org/reports/business_case_for_ei.html

Cooper, D. L., Healy, M. A., \& Simpson, J. (1994). Student development through involvement: Specific changes over time. Journal of College Student Development, 35(2), 98-102.

Cooper, R. K. \& Sawaf, A. (1997). Executive EQ: Emotional intelligence in leadership and organizations. New York: Perigree Book.

Conger, J. (1989). The charismatic leader: Behind the mystique of exceptional leadership. San Francisco: Jossey-Bass.

Costa, P. T., Terracciano, A. \& McCrae, R. R. (2001). Gender differences in personality traits across cultures: robust and surprising findings. Journal of Personality and Social Psychology, 81(2), 322-331.

Davies, M., Stankov, L. \& Roberts, R. D. (1998). Emotional Intelligence. Journal of Personality and Social Psychology, 75(4), 989-1015. 
Day, D. (2001). Leadership development: A review in context. Leadership Quarterly, 11 (4), 581-613.

Dugan, J. P. (2006). Explorations using the social change model: Leadership development among college men and women. Journal of College Student Development, 47(2), 217-225.

Dugan, J. P. \& Komives, S. R. (2007). Developing leadership capacity in college students: Findings from a national study. College Park, MD: National Clearinghouse for Leadership Programs.

Dugan, J. P., Komives, S. R. \& Segar, T. C. (2008). College student capacity for socially responsible leadership: Understanding norms and influences of race, gender, and sexual orientation. NASPA Journal, 45(4), 475-500.

Eagly, A. H. \& Carli, L. L. (2007). Through the labyrinth: The truth about how women become leaders. Boston: Harvard Business School Press.

Eagly, A. H., Johannesen-Schmidt, M. C. \& van Engen, M. L. (2003). Transformational, transactional, and laissez-faire attitudes: A metaanalysis comparing women and men. Psychological Bulletin, 129, 569591.

Fiedler, F. (1972). The effects of leadership training and experience: A Contingency model interpretation. Administrative Science Quarterly, 17(4), 453-470.

Fiedler, F. (1995). Reflection by an accidental theorist. Leadership Quarterly, $6(4), 453-461$.

Fiedler, F. \& Mahar, L. (1979). A field experiment validating contingency model leadership training. Journal of Applied Psychology, 64 (3), 247254.

Gardner, H. (1983). Frames of mind. New York: Basic Books.

Goleman, D. (2000). Leadership that gets results. Harvard Business Review, March-April, 2000, 78-90. 
Goleman, D., Boyatzis, R. \& McKee, A. (2002). Primal leadership: Realizing the power of emotional intelligence. Boston, MA: Harvard Business School Press.

Goleman, D. (2000). Emotional intelligence: Issues in paradigm building. In D.

Goleman \& C. Cherniss (eds.), The Emotionally Intelligent Workplace: How to Select for, Measure, and Improve Emotional Intelligence in Individuals, Groups, and Organizations. San Francisco, CA: JosseyBass.

Goleman, D. (1998). Working with emotional intelligence. New York: Bantam Books.

Guastello, D. D. \& Guastello, S. J. (2003). Androgyny, gender role behavior, and emotional intelligence among college students and their parents. Sex Roles, 49(11/12), 663-673.

Haber, P. (2006). Co-curricular involvement, formal leadership roles, and leadership education: Experiences predicting college student socially responsible leadership outcomes. Unpublished master's thesis. University of Maryland, College Park, MD.

Haber, P. \& Komives, S. R. (2009). Predicting the individual values of the social change model of leadership development: The role of college students' leadership and involvement experiences. Journal of Leadership Education, 7(3), 123-156.

Heifetz, R. A. \& Linsky, M. (2002). Leadership on the line. Cambridge, MA: Harvard Business Review.

Heifetz, R. A. (1994). Leadership without easy answers. Cambridge, MA: Belknap Press of Harvard University Press.

Hernandez, K., Hogan, S., Hathaway, C. \& Lovell, C. D. (1999). Analysis of the literature on the impact of student involvement on student development and learning: More questions than answers? NASPA Journal, 36(3), 184-197.

Hunter. J. E., and Hunter, R. F. (1984). Validity and utility of alternative predictors of job performance. Psychological Bulletin. 760, 12-93. 
Higher Education Research Institute (HERI). (1996). A social change model of leadership development: Guidebook version III. Los Angeles: University of California Los Angeles: Higher Education Research Institute.

Jordan, P. \& Ashkanasy, N. M. (2006). Emotional intelligence, emotional self-awareness, and team effectiveness. In V. U. Druskat, F. Sala \& G. Mount (Eds.), Linking emotional intelligence and performance at work: Current research evidence with individuals and groups. Mahwah, NJ: Lawrence Erlbaum Associates.

Judge, T. A., Bono, J. E., Ilies, R. \& Gerhardt, M. W. (2002). Personality and leadership: A qualitative and quantitative review. Journal of Applied Psychology, 87(4), 765-780.

Kellerman, B. (2004). Bad Leadership. Boston, MA: Harvard Business School Press.

Kellerman, B. (2008). Followership: How followers are creating change and changing leaders. Boston, MA: Harvard Business Press.

Kelley, R. E. (1988). In praise of followers. Harvard Business Review, Nov.Dec., 142-148.

Kerr, R., Garvin, J., Heaton, N. \& Boyle, E. (2006). Emotional intelligence and leadership effectiveness. Leadership \& Organization Development Journal, 27(4), 265-279.

Kezar, A. \& Moriarty, D. (2000). Expanding our understanding of student leadership development: A study exploring gender and ethnic identity. Journal of College Student Development, 41(1), 55-69.

Komives, S. R., Lucas, N. \& McMahon, T. (2007). Exploring Leadership: for college students who want to make a difference. ( $2^{\text {nd }}$ ed.). San Francisco, CA: Jossey-Bass.

Komives, S. R. \& Wagner, W. (2009). Leadership for a better world: Understanding the social model of leadership development. San Francisco, CA: Jossey-Bass. 
Komives, S. R., Dugan, J. P., Owen, J. E., Slack, C. \& Wagner, W. (Eds.). (2006). Handbook for student leadership programs. College Park, MD: National Clearinghouse for Leadership Programs.

Kouzes J. \& Posner, B. (2007). The leadership challenge: How to keep getting extraordinary things done in organizations ( $4^{\text {th }}$ edition). San Francisco, CA: Jossey-Bass.

Kouzes J. \& Posner, B. (2008). The student leadership challenge: Five practices for exemplary leaders. San Francisco, CA: Jossey-Bass.

Lopes, P. N., Salovey, P. \& Straus, R. (2003). Emotional intelligence, personality, and the perceived quality of social relationships. Personality and Individual Differences, 35, 641-659.

Lowney, C. (2003). Heroic leadership best practices from a 450-year-old company that changed the world. New York: Loyola Press.

Mandell, B. \& Pherwani, S. (2003). Relationship between intelligence and transformational leadership style: A gender comparison. Journal of Business and Psychology, 17(3), 387-404.

Marshall, S. M. \& Hornak, A. M. (2008). A day in the life of a college student leader: Case studies for undergraduate leaders. Sterling, VA: Stylus Publishing.

Mayer, J. D., D. R. Caruso, and P. Salovey (2000). Selecting a measure of emotional intelligence: The case for ability scales. Handbook of Emotional Intelligence: 320-42. New York: Jossey-Bass. Print.

McCauley, C. D. \& Van Velsor, E. (Eds.). (2005). The center for creative leadership handbook of leadership development. San Francisco: Jossey-Bass.

McClelland. D. C. (1973). Testing for competence rather than intelligence. American Psychologist, 28, 1-14.

Melia, M. and Heckerman, D. (1998). An experimental comparison of several clustering and initialization methods. Microsoft Research Technical Report, MSR-TR-98-06. 
Northouse, P. G. (2009). Introduction to leadership concepts and practice. London: Sage.

Northouse, P. G. (2004). Leadership: Theory and practice (3rd ed.). Thousand Oaks, CA: Sage Publications.

Roberts, D. (Ed.). (1981). Student leadership programs in higher education. Carbondale, IL: Southern Illinois University/American College Personnel Association.

Romano, C. R. (1996). A qualitative study of women student leaders. Journal of College Student Development, 37(6), 676-683.

Salovey, P. \& Mayer, J. D. (1990). Emotional intelligence. Imagination, Cognition and Personality, 9 (3), 195-211.

Sax, L. J. (2009, May-June). Gender matters: the variable effect of gender on the student experience. About Campus, 2-9.

Seligman, M. E., (1998). Learned Optimism: How to Change Your Mind and Your Life. New York: Pocket Books.

Shankman, M. L. \& Allen, S. J. (2008). Emotionally intelligent leadership: A guide for college students. San Francisco: Jossey-Bass.

Shankman, M. L., Allen, S. J., Facca, T. M. (2010). Emotionally intelligence leadership for students: Inventory. San Francisco: Jossey-Bass.

Shankman, M. L., Haber, P., Facca, T. M. \& Allen, S. J. (2010). Gender and leadership through the lens of emotionally intelligent leadership. Submitted to Leadership Review, 16 February 2010

Singh, D. (2006). Emotional intelligence at work: A professional guide $\left(3^{\mathrm{rd}}\right.$ ed.). New Delhi, India: Response Books.

Spencer, L. M. J. et al. (1997). Competency assessment methods: History and State of the Art. Boston: Hay/McBer.

Stemberg, R. (1996). Successful intelligence. New York: Simon and Schuster. 
Strube, M. \& Garcia, J. (1981). A meta-analytical investigation of Fiedler's Contingency Model of leadership effectiveness. Psychological Bulletin, 90, 307-321.

Theodoridis, S. \& Koutroumbas, K. (1996). Pattern recognition. New York: Academic Press.

Thomdike, R. L. \& Stein, S. (1937). An evaluation of the attempts to measure social intelligence. Psychological Bulletin, 34, 275-284.

Weisinger, H. (1998). Emotional intelligence at work. San Francisco: Jossey-Bass.

Zhang, T., Ramakrishnon, R. \& Livny, M. (1996). BIRCH: Method for very large databases. Proceedings of the ACM Management of Data, 103114. Montreal, Canada. 


\section{Classification and Analysis of Franchise Resource Data}

\subsection{Franchising market - Issues, Constraints and Literature review}

Over 30 years ago, investigating the effects of tying agreements on the franchise system of distribution, Hunt and Nevin (1975) suggested that legislation and franchisee vs. franchisor litigation had proliferated to crisis proportions. Legal expert Glosband (1972) believed the problem of restrictions on the source of supplies or services was at the heart of virtually every complaint pending against franchisors.

This work initially investigates satisfaction with services provided by the franchisor to the franchisee, as well as overall satisfaction levels. These services can be considered marketing services in that the programs are aimed at assisting the franchisee in generating revenue. Such programs, products and services are typically provided by the franchisor to the franchisee as revenuegenerating tools, thus increasing royalty fees paid to franchisor. The aim then is to use analytical techniques to better understand franchise market segments and make suggestions for more effective allocation of franchisor resources. Analytical methods are applied that may potentially reduce franchisee/franchisor conflict by understanding franchisees' perceptions of effectiveness, value and satisfaction with franchisor programs and services. Such understanding can be used by the franchisor to guide lesser performing franchisees. In effect, the franchisor can investigate the behaviors of higher performers, design programs that are reflective of these behaviors, and implement them with lower performing franchisees, with the intent of generating increased revenue and/or enhanced performance.

Two primary streams of research are found in the franchising literature, the first being a more theoretical discussion of owning versus franchising, and the second investigating factors that affect royalty fees (Agrawal and Lal, 1995). Literature specifically addressing marketing issues affecting the franchise relationship is limited. Marketers in a franchised organization are vulnerable to criticism for a number of reasons including misguided strategic decisionmaking at higher levels, misperceptions of effectiveness, and miscommunication, particularly in large franchises where messages are often stopped at the franchisee-owner level, and not disseminated down to the operational level. Further, marketers often fail to communicate sufficient quantitative evidence required to garner system-wide participation in programs. 
Bridging the Marketing-Finance Gap in a Franchise System of Distribution

In the past decade, there has been an explosion of literature in marketing theory about validating marketing's impact (Rao, 2008; Doyle, 2000; Srivastava, 1998) and the link between customer satisfaction and shareholder value (Aksoy, 2008; Luo, 2008).

In 2001, at the forefront of a then-impending dot.com meltdown, Shaw editorialized that marketing and finance needed to agree on a common measurement framework (Shaw, 2001). His summary insights and recommendations at the time were clearly reflective of the instability of the marketing function with regard to financial responsibility. Shaw suggested,

"More progressive finance directors are driving towards a more integrated framework. They have taken on the customer and competitive measurement problem themselves, something which entails creating several new roles. Market research is moved into the finance department, thus guaranteeing a degree of objectivity not found when marketing runs market research. Predictive modeling also gets commissioned by the finance department, either by using outside consultants or in-house analysts. Balanced scorecards are developed by the finance department: marketing objectives and strategies thus are quantified in ways that are rare when marketing are responsible for marketing plans."

While Shaw's comments may slice through the core of a marketer's heart, his position is warranted. In 2003, Tim Ambler addressed the marketing-finance gap issues, questioning whether the over-popularized philosophy of managing for shareholder value was assisting or damaging the generation of inward cash flow (Ambler, 2003). He suggested that the analysis of shareholder value is a worthwhile technique for identifying assets and costs that can be adjusted, but when used as a 'universal framework for decision making', it may be misleading, particularly for marketing measurements. Typically, finance does not look at future returns as a result of brand building activities today, nor does it evaluate the financial impact of marketing activities from a perspective that acts as a managerial control mechanism on specific marketing activities.

Ambler also suggested using quantitative metrics in a way that leads to effective action. Day (1990), suggested that effective use of metrics includes linking marketing activities to market response and then to subsequent financial impact.

In 2002, between Shaw's lambasting and Ambler's tactful rebuttal (the two not necessarily related), marketing scholars (Ambler, Carpenter, Jacobson, Kumar, Rust, Srivastava, 2004) responded with a partnership between the 
American Marketing Association and the Marketing Science Institute to research the need for bridging the metrics gap between marketing and finance.

A special section of the Journal of Marketing in 2004 was the result of a push for more research on the connection between marketing and finance from the Marketing Science Institute. In the introduction to this issue, Lehmann (2004) suggested that although measurements of return-on-investment or margins are accepted standards, in order to accurately assess marketing performance these measures need to be used in conjunction with financial measures of the value of marketing assets that have long-term value such as brand equity, or customer attitudes, behaviors and other evaluative criteria. Acknowledging the data and methodological issues and constraints related to developing an accurate value chain (or the bridge between customer associations and stock price), Lehmann asserted,

"... an attempt to lay out such a value chain and estimate the links seems to be critical. This means there can be no overreliance on a single measure...but there needs to be measures at all the levels. Put simply, if marketing wants " $a$ seat at the table" in important business decisions, it must link to financial performance."

Without a seat at the table, Lehmann predicted that marketing would lose ground to other areas such as product development, and devolve into departments of "ad copy and cents-off coupons" (Lehmann, 1996).

The collaborative efforts by Rust, Ambler, Carpenter, Kumar and Srivastava (2004) suggest that a lack of accountability among marketers has undermined their credibility. Without evidence of the impact of marketing effectiveness on shareholder value, the marketer's very existence in the organization is threatened. Rust, et. al., suggest that it is possible to show how marketing expenditures add to shareholder value. Dissemination of methods of assessing marketing productivity to the business community serves to validate marketing's position within the firm. They recommend dynamic models that link tactics to financial impact. Research and models are necessary in many areas, including strategies and tactics, brand equity, customer equity, market impact, financial impact, the environment and competition.

While academics busily collected, evaluated, and honed the available analytical techniques appropriate for measuring and validating marketing effectiveness, executives in finance began to realize that the union of finance and marketing was essential to spawn growth initiatives from within (organic growth) vs. acquiring other companies. Earnings at industry leaders like Best Buy Co., Inc., Genetech Inc., Barclays PLC and Roche Holding AG, all stock- 
market stars in the recent years prior to 2005, came primarily from organic growth. Further, companies in the top quartile of organic growth are 13 times more likely to be in the top quartile of shareholder returns (Jenkins and Meer, 2005).

Practitioners recognize inherent challenges in bridging the gap between finance and marketing, particularly the obvious differences in perspectives. Where finance is motivated by measurement, stability and that which is known, marketing thrives on vision, creativity and innovation. Jenkins and Meer (2005) suggest that requests for budget increases rarely explain how they will boost revenue and profitability. Barclay's former finance director for private client business, Mike Murray suggests marketing's lack of explanation leads to finance "taking the easy way out and cutting marketing spending instead of finding less productive costs that should be reduced to meet profit targets." Jenkins and Meer point to innovation, evaluation of growth opportunities and portfolio management as areas in which a strong marketing and finance partnership can drive firm benefits. Finance executives can help marketers better evaluate the top and bottom line impact of their plans and secure funds for their programs.

Confirming the seriousness of the issue, a 2005 study by Forrester Research, Marketing Management Analytics, and the Association of National Advertisers (ANA) found that only one in three marketers surveyed said they build marketing budgets based on knowledge of the spending required to meet corporate goals. If faced with a $10 \%$ budget cut, only $40 \%$ felt they could predict the impact of the cut on sales (See, 2006).

A common language between marketing and finance is integral to improving the alignment between the two functions. Ed See (2006) suggests that language should include marketing based drivers, such as consumption, distribution, displays and retailer publication features and their supporting data. Interviewing the VP Finance for a Fortune 500 cosmetics company, See reports that the company's financial projections are the outcomes of their marketing plan, and are thus closely linked. The VP Finance says, "We need to quantify, track and measure everything that we can, including marketing activity. Marketing is not just art - it can also be science, thus bringing it closer to a numbers function like finance."

Both academics and practitioners recognize the need for marketing metrics that satisfy the firm's financial reporting requirements, as well as accurately predict future market behaviors and potential responses to changes in firm strategy. Perhaps the complexity of academic models inhibits general acceptance by managerial practitioners. As Wübben and Wangenheim (2008) 
suggest, while stochastic customer base analysis models, designed by academics, are available, managerial application is not widespread.

The good news is that there are ample models and methods designed to measure marketing effectiveness. Just as an example, Srinivasan, Pauwels, Silva-Risso and Hanssens (2009) examined how product innovations and marketing investments for innovations lift stock returns by improving the outlook on future cash flows. They found adding 'information about marketing actions to the established finance benchmark model greatly improves the explained variance in stock returns.' Trusov, Bucklin and Pauwels add substantial insight to the effects of word-of-mouth (WOM) versus traditional marketing. Their method utilizes a vector autoregressive (VAR) modeling approach to show that WOM referrals have "substantially longer carryover effects and produce substantially higher response elasticities. They quantify the monetary value of a WOM referral, based on revenue from advertising impressions served to a new member. These innovative techniques and methods for quantifying the impact of marketing efforts are available but have not yet found their way into average marketing departments.

Rust et al (2004) suggest a greater emphasis on developing aggregate-level models that link tactics to financial impact. Further, they recommend research in the area of accounting for customer heterogeneity. Specifically, they mention the identification of high-profit customers as being central to market segmentation, strategic marketing and related tactics. The marketing scholars summarize their review suggesting that the evaluation of marketing productivity ultimately involves projecting the differences in cash flows that will occur from implementation of marketing action. An understanding of how marketing actions impact financial assets is likely to transform the way businesses are managed.

\section{Marketing and Resource Allocation Challenges in a Franchise Organization}

The franchisor faces substantial challenges related to resource allocation regarding franchisees. New members of the franchise system require substantial training resources, while more tenured franchisees typically seek assistance with revenue generating programs such as sales incentive contests, cooperative advertising or perhaps financing options for renovations or development. The franchisor's challenge is in identifying the franchisees that hold the most promise for future revenues, then allocating the appropriate resources. . 
Eliashberg and Michie (1984) investigated how multiple business goal sets determined marketing channel conflict. They highlight the fact that in many marketing distribution systems the channel members operate under different business philosophies. Wittreich (1962) suggested that independent owneroperators do not typically share the growth philosophy that corporate managers do. This individual's business philosophy is oriented toward reaching a point and leveling off into a continuously satisfying plateau. This is reflected in the franchising dataset in the segment nicknamed "Glory Days". This group of franchisees have been with the organization over 16 years and are in the lowest revenue category, earning less than $\$ 500,000$ in annual revenues. This segment reveals low levels of satisfaction with the franchisor, comparable to their higher revenue counterparts of the same tenure. Resource demands placed on the franchisor by this segment can diminish productivity without the financial return that can be anticipated from higher revenue segments.

Eliashberg further suggests that two different business philosophies are likely to result in two different business goals sets in many marketing channels - the overall system goals set and the individual members' informal but operationally tractable business goals set. The franchisor is the channel administrator who sets the overall system goals, but franchisees are likely to have different operative goals (Perrow, 1970). Usually, these operative goals are actively being pursued by the organization's members and the pursuit is reflected in the members' activities and resource allocation patterns (Connor and Bloomfield, 1970). It is fair to assume that different goal sets between the franchisor and franchisee can be a source of conflict resulting in lower levels of satisfaction.

Franchisors typically have a goal of standardizing the marketing practices of franchisees (Anand, 1987). The goal is usually addressed in franchise agreements, but federal, state and local regulations preserve the franchisees' discretion in making certain decisions. The franchisor must then be highly persuasive to generate franchisee support for programs. Anand and Stern (1985) found that franchisees would relinquish control when

- profitable decisions initiated by the franchisor were attributed to internal characteristics of the franchisor, such as ability and effort

- unprofitable decisions initiated by the franchisor were attributed to factors external to the franchisor, such as store location and luck

- their own profitable decisions were attributed to external factors, and 
- their own unprofitable decisions were attributed to internal causes, such as lack of ability or effort.

Anand (1987) suggests that management consider using subsamples to influence lower performers to act like their higher performing counterparts. If, for example, the "top $10 \%$ " is revealed to have relinquished control to the franchisor (implying participation in programs and services), then this news should be spread within the organization, hopefully garnering support for the franchisor's decision making skills. Channel members who perceive they are doing well (or badly) on their own (or the franchisor's decisions) might be influenced by performance of the top $10 \%$ or bottom $10 \%$.

In the dataset analyzed here, it can be seen that higher level performers participate in franchisor meetings, they perform benchmarking against other channel members, and share best practices. The analysis digs deeper to understand the specific programs and services that yield usage and satisfaction from the higher level performers. Thus, the lower levels can be encouraged to participate in a like manner, with the intent of moving them upward in profitability segments. 


\subsection{Description of the Dataset - Franchise Community Survey}

The subject organization is a 40 -year-old franchise network of employment recruiting service offices, with an estimated 1100 offices in 40 countries (800 in the United States, 300 international). Franchise offices target employers, contracting their services to find, pre-screen and present potential candidates for positions within the employer's organization. An average fee of $30 \%$ of the recruit's first year salary is paid by the employer to the franchise office for the aforementioned services. A fee of approximately $7.5 \%$ of top-line sales revenue is paid by the franchisee to the franchisor who offers no competing recruiting services, only support services to the franchisees. Franchises are sold by geographic area, but each works in an industry niche with no boundaries, borders or competitive protection resulting from the franchise agreement.

The dataset was collected in March 2009, with the following objectives defined:

- Generate valid and reliable research from members of the franchise community that will enable the effective design of a strategic plan for growth that is perceived as equitable and valuable to both the franchisor and its franchisees.

- The research investigates services provided by the franchisor including operations, marketing, training and information technology, and the levels of utilization and satisfaction within the franchise community.

The dataset was collected via a web-enabled survey, introduced and sent in an email as link in late January 2009. The invitation to participate was sent to the entire franchise network in the United States, yet only approximately 300-400 franchisees are considered active participants in the franchise community. Varied size and tenure offices are represented in the final sample of 225 respondents. A total of 447 variables measured, with over 100,000 data points considered in the initial analysis reported to the franchisor. This work will discuss only the most relevant areas investigated, those that are utilized in the final discriminant analysis.

Nearly $30 \%$ (26.7\%) of the respondents had been with the network for over 20 years. Varied tenure of franchise ownership were comparably represented, with the exception of those in business $1-3$ years (10\%) and less than one year (4\%), see Figure 1.2.1. 


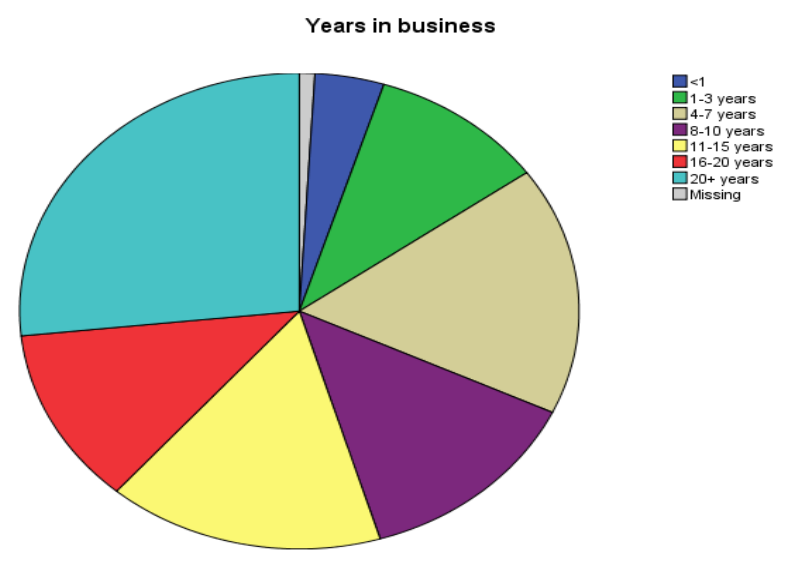

Figure 1.2.1 Franchisee tenure distribution

Forty percent reported less than $\$ 500 \mathrm{~K}$ in annual revenues, followed by nearly $25 \%$ (24.4) in the $\$ 1-3 \mathrm{MM}$ range. Another 18\% reported average annual revenues at $\$ 500,000-\$ 750,000$ (Figure 1.2.2).

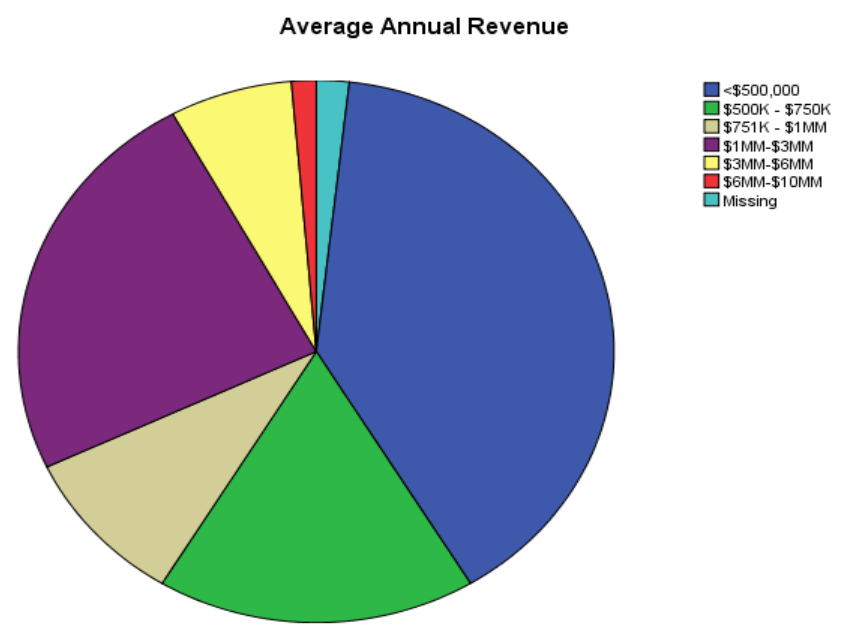

Figure 1.2.2 Franchisee sales distribution

Thirty-seven percent employed 1-3 staff members (including themselves), and another 33\% ran their business with 4-7 employees (Figure 1.2.3). Thirteen percent of respondents managed their office with a staff of $8-10$, and $7 \%$ had twenty or more staff. 


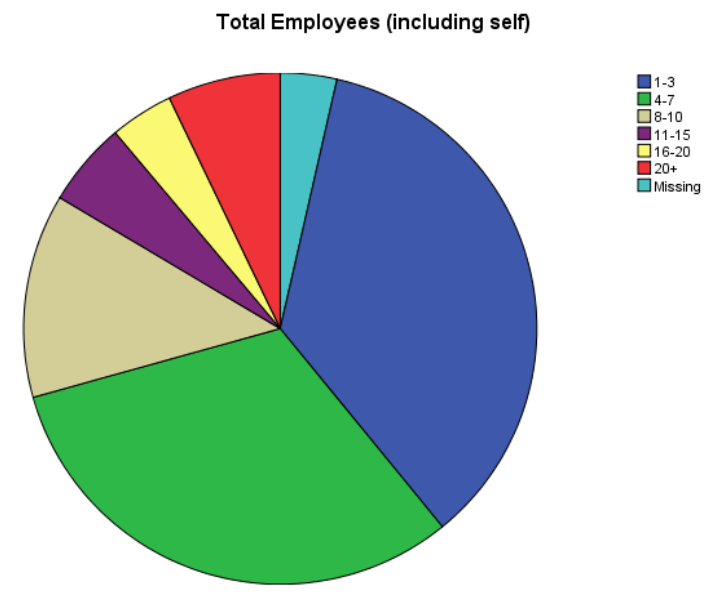

Figure 1.2.3 Number of employees in franchisee office

Satisfaction with functional areas was assessed, indicating overall satisfaction with franchisor programs and services was rated at 5.44 on the $1=$ low to $10=$ high satisfaction scale. Legal services (7.48) and Learning and Talent Development (6.5) were rated at the top whereas marketing and vendor programs rated lowest, just above overall satisfaction (Figure 1.2.4).

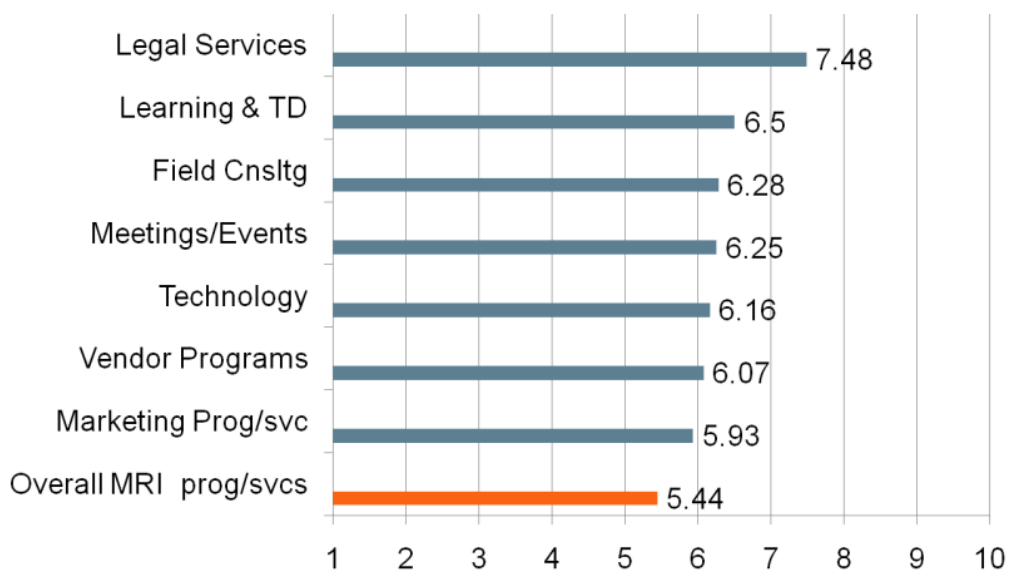

Figure 1.2.4 Franchisee satisfaction with functional areas of service; overall satisfaction with franchisor

Satisfaction regarding specific programs and services was highest with office supplies and services at 6.52 , followed by The Resource at 6.5 . This is the website database for all franchise-related documentation including marketing and training materials, communications, benchmark ratings, etc. Revenue generating programs were rated alarmingly low at 3.76 (Figure 1.2.5) 


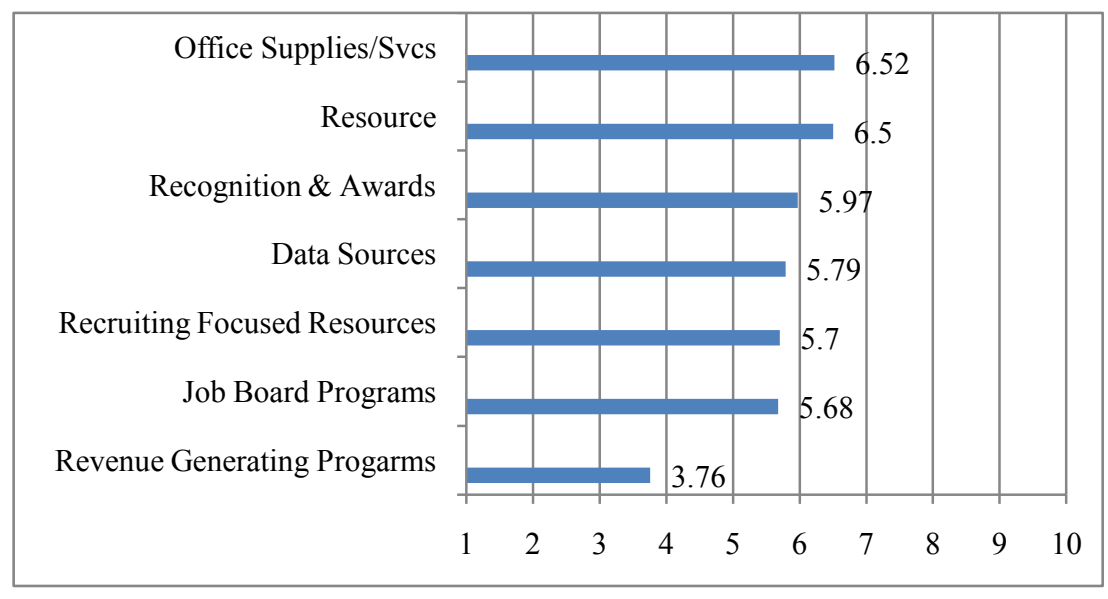

Figure 1.2.5 Franchisee satisfaction with specific programs and services provided by franchisor

Satisfaction with field consulting services was rated at 6.28 on the 10 -point scale. These services typically involve telephone calls, emails and personal office visits from representatives of the franchisor. The representative provides guidance, direction, and assistance with all matters related to managing and developing the business in the prescribed manner suggested by franchisor management. Use and satisfaction with these services are integral to value perceptions perceived by franchisees. Seventy-four percent report usage of field services. Note that the "aware" box was checked by $57 \%$, but we infer that if one used, they must be "aware". Responsiveness was rated fairly high at 7.92, while value perceptions, effectiveness and future usage intent hover near 7 on the 10 point scale.

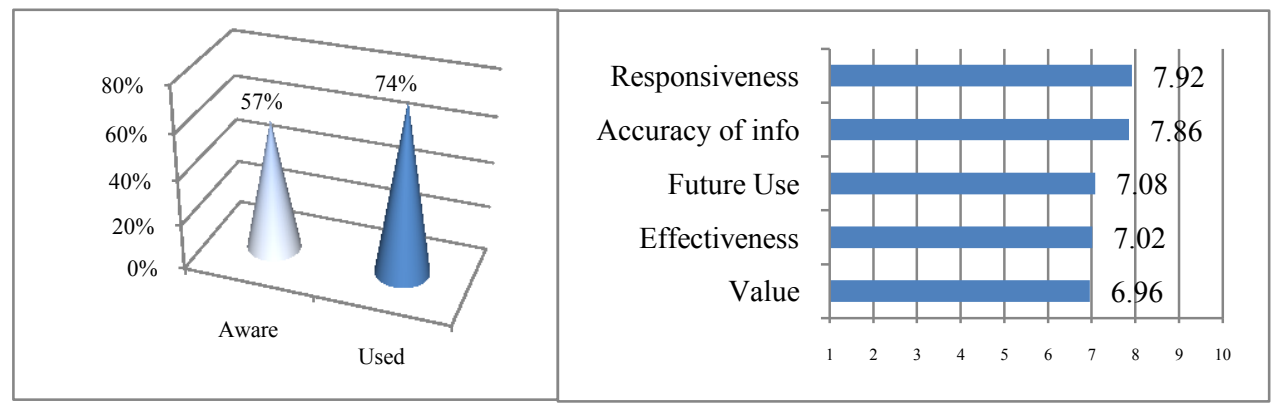

Figure 1.2.6 Field consulting services - overall satisfaction 6.28

While a substantial $83 \%$ reported awareness of public relations services, only $20 \%$ had used them. Information was rated relatively accurate as this is primarily supplied by participating franchisee. Responsiveness was rated at 6.15 , value perceptions were not strong and intent of future use of public relations services rather limited at less than 5 (Figure 1.2.7). 


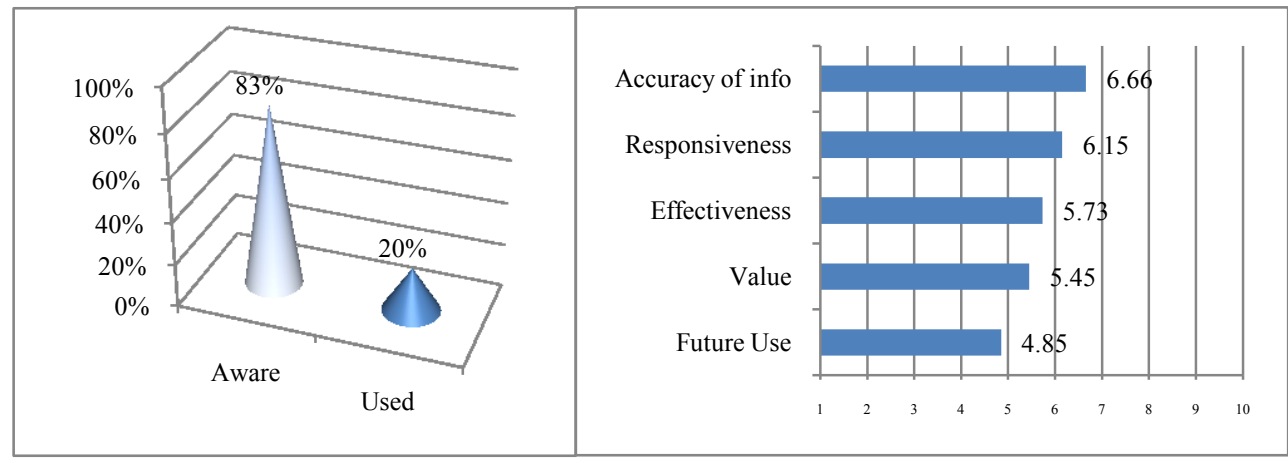

Figure 1.2.7 Public relations services - usage, value perceptions and intent of future use low

Assuming awareness if used, 90\% of the respondents reported using the "Resource", the website for franchise information. Satisfaction with the information website was rated at 6.5 , with intent of future use fairly high at 8.46 and value perceptions relatively strong at 7.27. Information was perceived to be acceptably accurate at 7.24 (Figure 1.2.8).

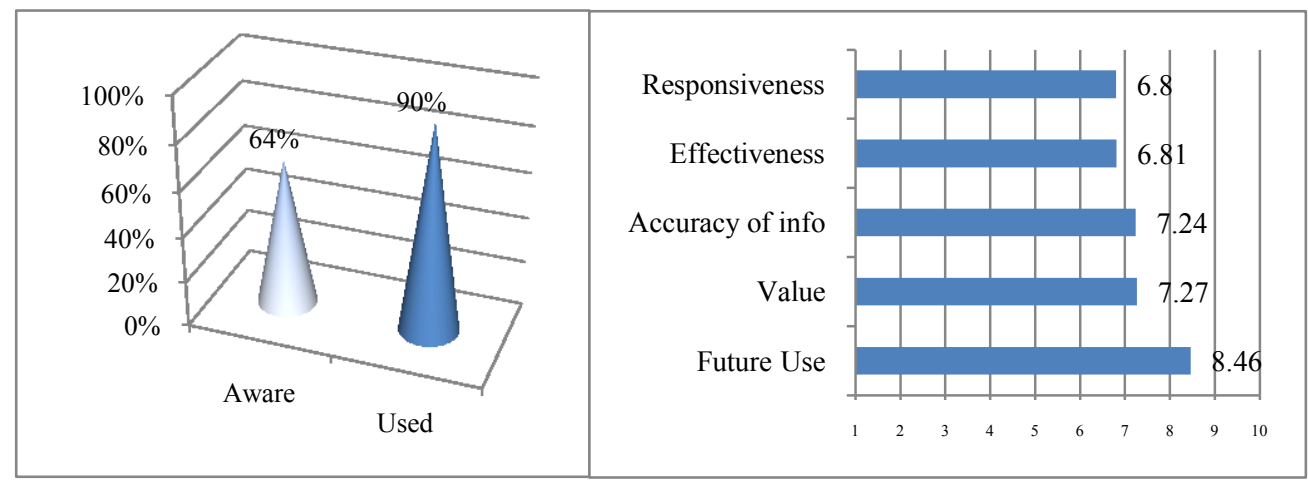

Figure 1.2.8 The Resource, franchise information website; usage, value perceptions, future use intent 
Training and development programs offered by the franchisor managed a 6.5 satisfaction rating. In general, awareness of the training and development programs was strong, ranging from roughly $60-80 \%$. Training videos were the

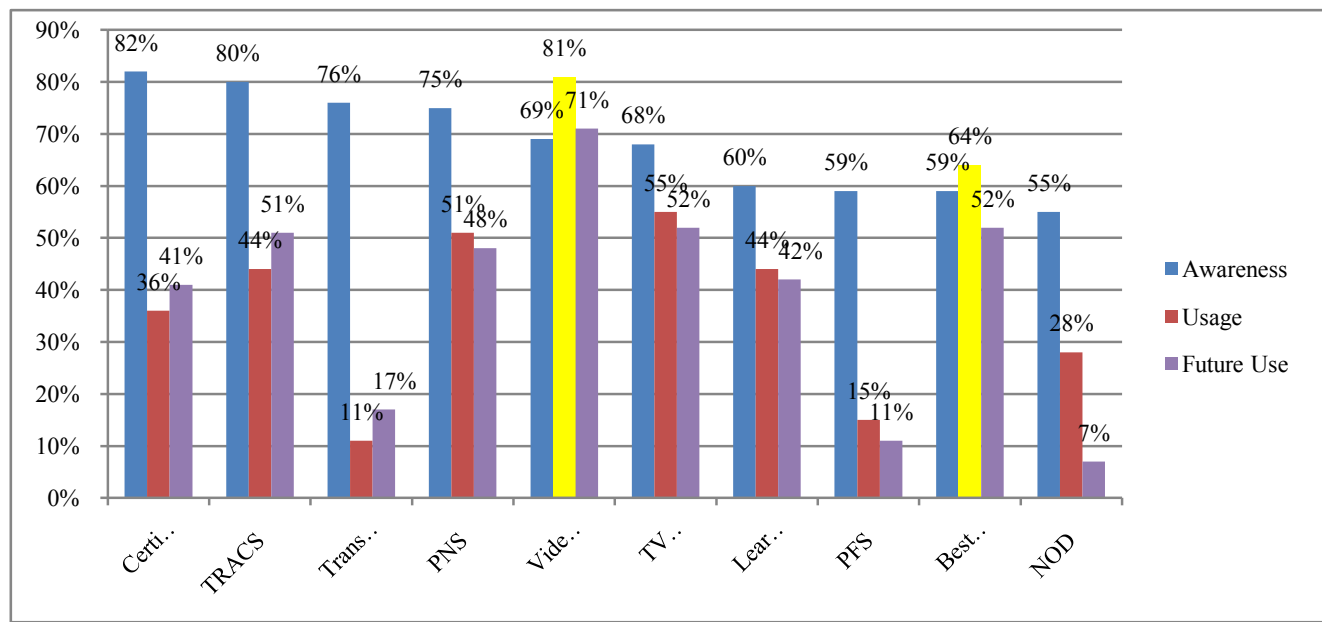

Figure 1.2.9 Awareness, usage and intent to use training materials in the future. Videos and best practice sharing most utilized resources

Best Practice Sharing results in the strongest value perceptions at 8.19, followed by the franchisor's online television-type resource at 8.13 , and the Video Vault at 8.02 (Figure 1.2.10). These same tools are considered the most effective as well. The training resources are similar in that they all serve as "how to" resources for recruiters, but the media vehicles differ. Typically a recruiter might watch the franchisor's "TV" network online, downloading video clips of presentations delivered at former meetings, workshops and seminars. The videos in the "vault" may be older, but still worthwhile and shared in an office group meeting as a training element. Sharing best practices happens in all these situations, yet this typically implies learning from an "expert" colleague within the franchise community or within one's own office.

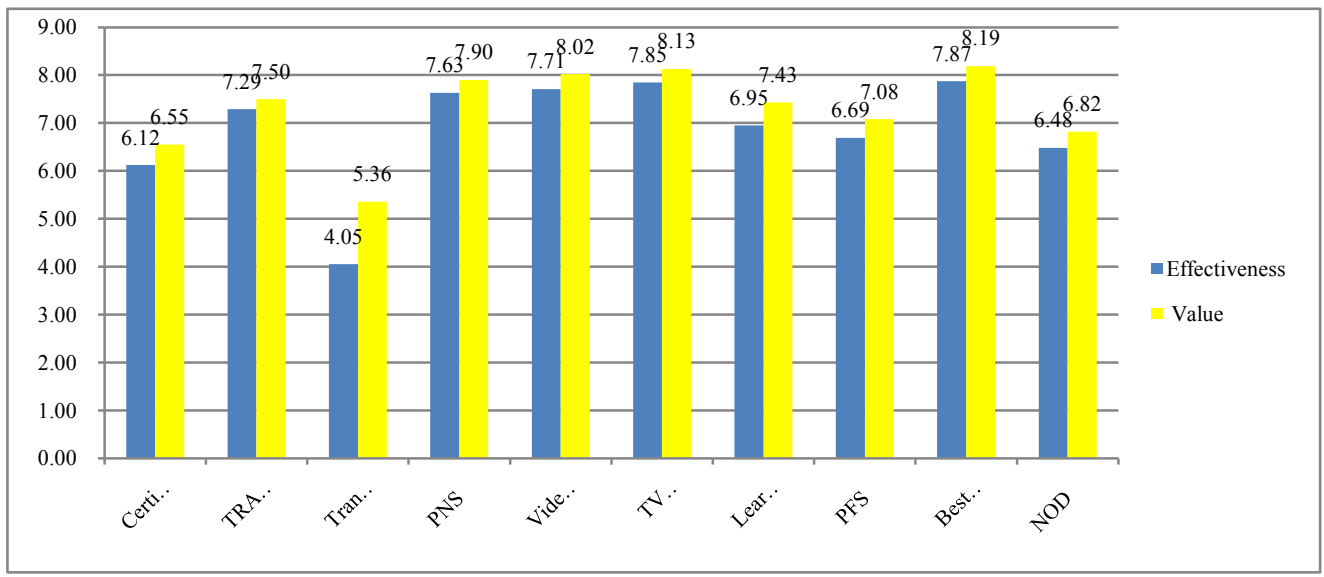

Figure 1.2.10 Franchisee's effectiveness \& value perceptions regarding training and development programs 
Assuming awareness, usage of the global conference and regional meetings were nearly $90 \%$, yet future intention of use was only $64 \%$ for the global conference and $75 \%$ for regional meetings (Figure 1.2 .11 )

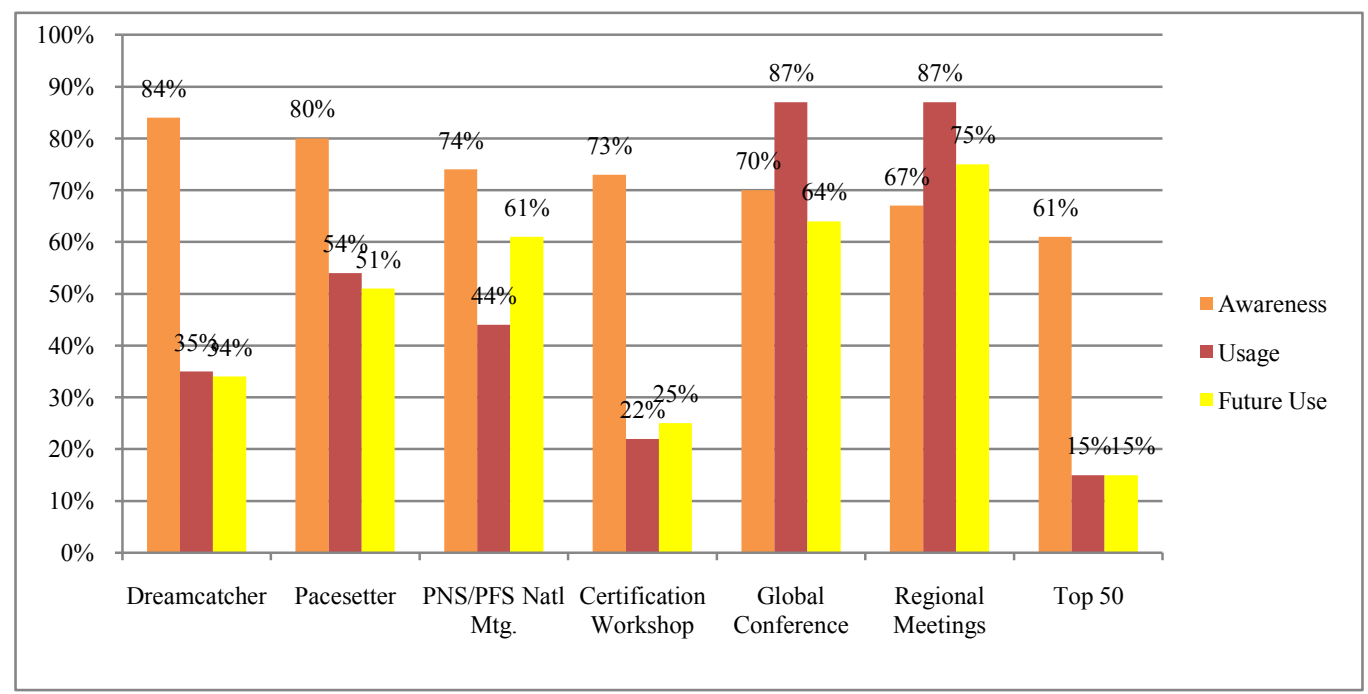

Figure 1.2.11 Franchisees' awareness, usage and intent of future use of franchisor sponsored meetings and events

For regional meetings, value perceptions were rated 7.18 and global conference at 6.54. As far as effectiveness, regional meetings were rated highest at 7.29, and the global conference perceived as fairly effective at 6.8, with value perceptions slightly lower at 6.54 (Figure 1.2.12).

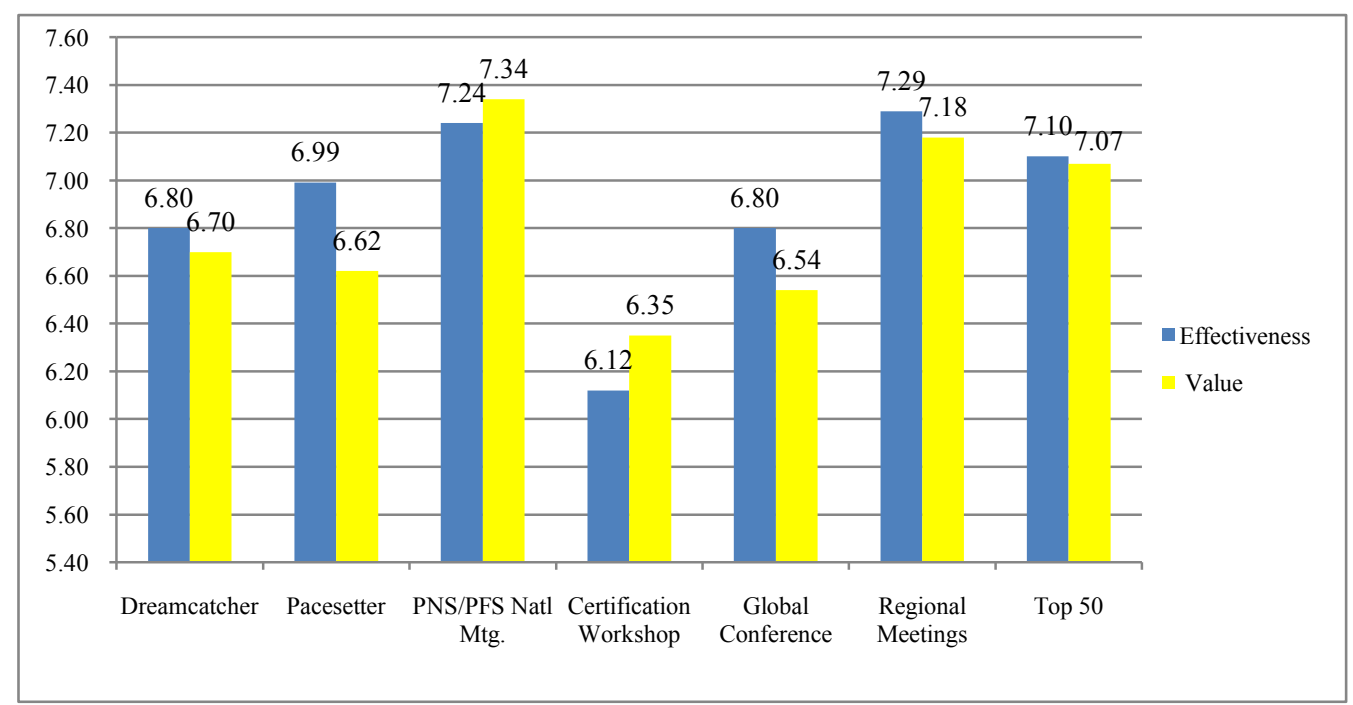

Figure 1.2.12 Franchisees' effectiveness and value perceptions for meetings and events 
Breaking out the system into roughly thirds, (low, mid, high) on revenue and tenure, it can be seen that satisfaction erodes as revenue increases. The midlevel 8-15 year old offices report the lowest level of satisfaction (Figure 1.2.13) Interestingly however, there is no significant correlation between satisfaction with the franchisor's programs and services and either revenue or tenure. There is a slight, but significant, relationship between tenure and revenue.

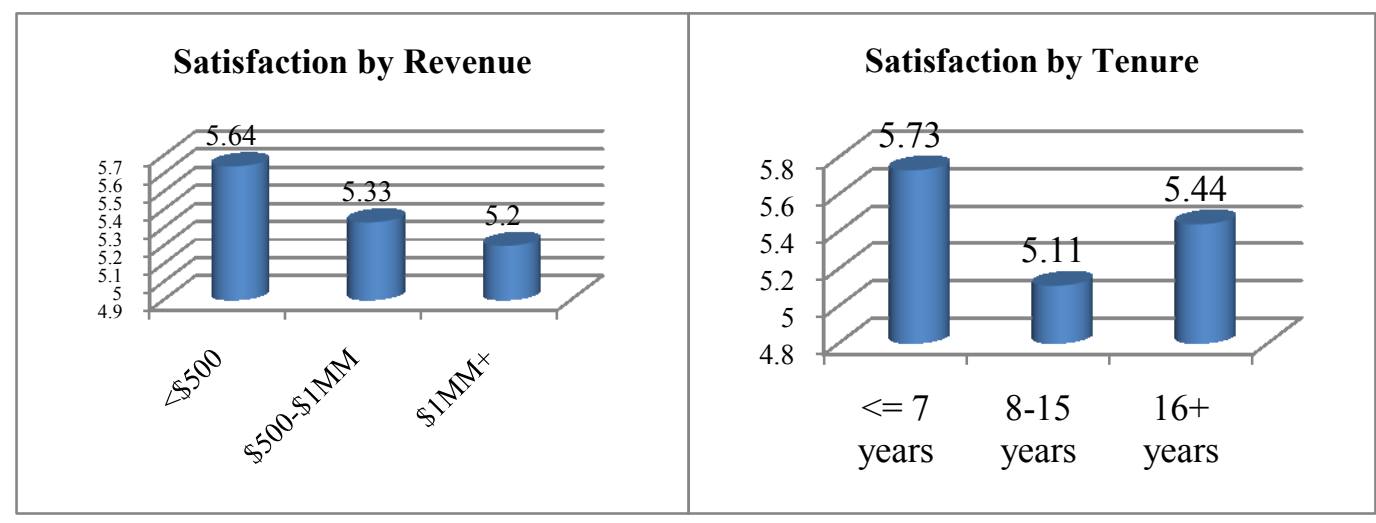

Figure 1.2.13 Franchisee satisfaction by revenue segment and by tenure

Overall, the description of the dataset allows a glimpse into the satisfaction problem of a large, well-established franchise organization in a service industry. Without the tangible assets often found in successful franchises, such as secret recipes, brand equity, or national advertising campaigns, maintaining franchisees' value perceptions and subsequent satisfaction with the resources provided by the franchisor, is challenging at best. This leads to a discussion of the managerial problem at hand. 


\subsection{General problem - where to focus franchisor's resources?}

Given that the franchise organization under analysis was established over 40 years ago, there is a rich history of recruiting skills required to execute effectively, and techniques to apply in virtually any situation (e.g., candidate wants more money, job description changes after recruiting has begun, etc.). As evidenced in the previous section, satisfaction declines as revenue increases (see Figure 1.2.13). Recall, however that there is no evidence that an individual franchisee's satisfaction decreases over time, but if one considers the segments utilizing the services of the franchisor, it is clear that franchisees with revenues under $\$ 500,000$ hold the highest level of satisfaction, as do those who have been in the franchise system for seven years or less. The implication is that the franchisee "outgrows" the resources of the franchisor as tenure and revenue increase. Without addressing this vulnerability, the franchise organization remains unstable.

If the franchisor elects to address the questionable sustainability of growth, it must consider allocation of resources. Each franchise contract essentially promises the same support, regardless of tenure or revenue, in exchange for a franchise fee. Contracts are typically 10 years in duration. An assessment of renewed contracts and the status and satisfaction at the time of renewal would prove fruitful for future research, but the data are not available here. So, the question remains to be framed around the best use of both human and physical material resources to support the franchise network as a whole, yet providing ample individual opportunity for business development and growth at the franchisee office level. Top line revenue growth for the franchisee directly results in increased franchise fees, or revenues, paid to the franchisor.

Often times the reality is that struggling franchisees can be the most demanding on the franchisor's limited resources. Time and resources are spent on field visits, developing individual marketing and sales strategies, assisting with implementation, providing guidance, and encouraging progress toward these single-unit goals. The undertaking may increase the franchisee's satisfaction and value perception in the short-term, but the consistent strain on resources tends to outweigh the long-term effectiveness, and ultimately, the franchisor's revenue results. It is common in a franchise organization to have franchisor representatives serve anywhere from a 10:1 to 30:1 ratio of franchisees to franchisor representative. As the system grows, field service representatives tend to take on one or two new franchisees annually, depending on industry growth trends, but increasing staffing to support individual unit additions is seldom considered unless the growth rate exceeds obvious capacities. 
Thus, a plan for effective allocation of resources, including material resources, time, and human resources, is essential to the franchisor's growth strategy. A method of discerning the differences between the attitudes and behaviors of the highly successful versus those less productive franchisees, is integral to establishing a strategic plan for servicing the franchise community. While this work does not differentiate the types of resources allocated (time, human, material) it will enable a method for determining significant differences between franchisee performance levels, the resources they use and intend to use in the future, value and effectiveness perceptions, and satisfaction states of the varied segments.

Essentially, if the franchisor can understand the resources utilized and preferred by franchisees with better performance, preferably in a shorter period of time, it can focus on encouraging the use of similar resources by those in a less effective position, and potentially move them "upward" to a higher performance position. These segments and their preferences will be discussed in the next section. 


\subsection{Problem of classification - by tenure and revenue segments}

Franchisee respondents were segmented into like groups for comparative analysis. Nine groups were created based on two variables, annual revenue and tenure with the franchise. The low revenue segment includes franchisees with average annual revenue less than $\$ 500,000$. The mid-level revenue segment includes those with sales from $\$ 500,000$ to $\$ 1$ million. The high revenue group achieves an average annual revenue greater than $\$ 1$ million. Tenure with the franchise network was segmented as low if the franchisee had been in the system up to seven years; mid-level means 8 to 15 years, and high level are those in the system 16 or more years. Each group was assigned a name to reflect the segment to which they belonged, based on revenue and tenure. The following chart provides the segment name, percentage of respondents in the segment, and that segment's overall satisfaction with the franchisor's programs and services (Figure 1.4.1).

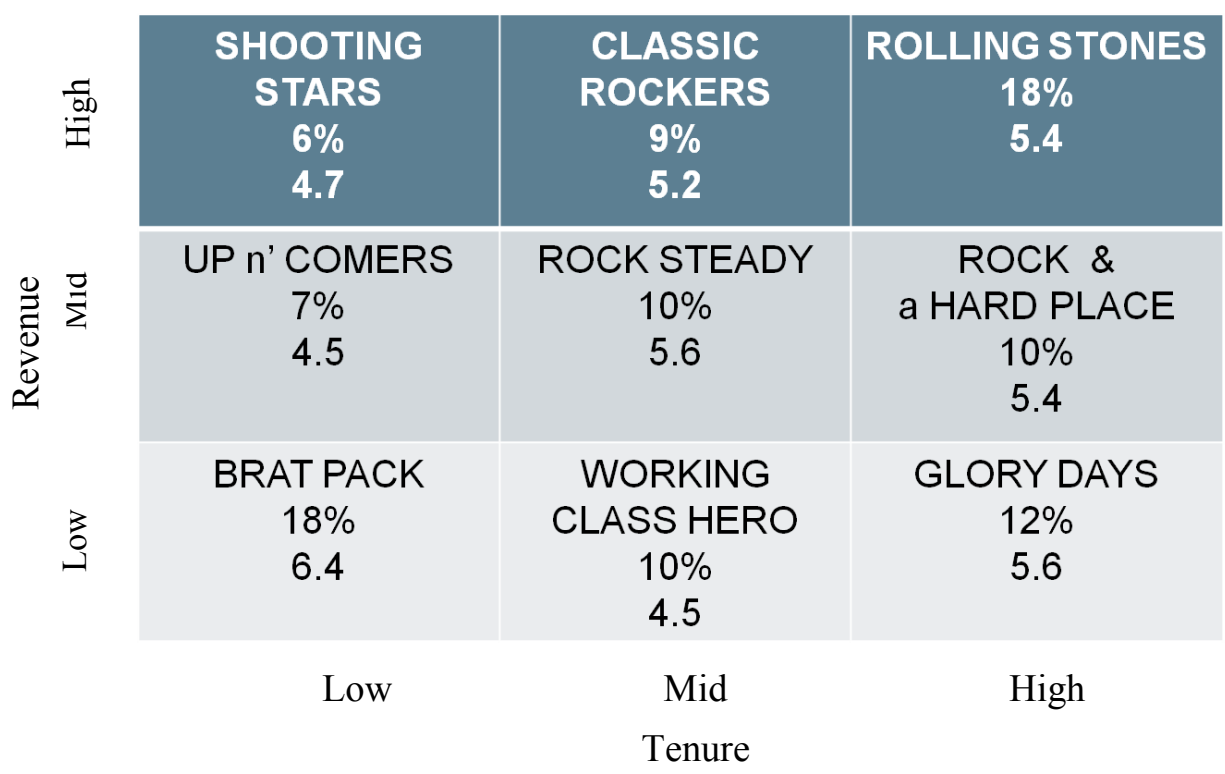

Figure 1.4.1 Franchisee segments, percentage in segment and satisfaction with franchisor (1 to 10 scale)

\section{Resource Requirements by Segment}

Next investigated were the resources each segment intended to use in the future. Intent to use some of the products and services provided by the franchisor was rated on a 1 to 10 scale which is depicted in the upper right of the following figures. In most instances intent to use a given product or service was indicated by marking a check box, thus the most common frequency figures are reported in the lower left of the figure. Figure 1.4.2 explains how to read each of the upcoming figures for resource requirements by segment. 


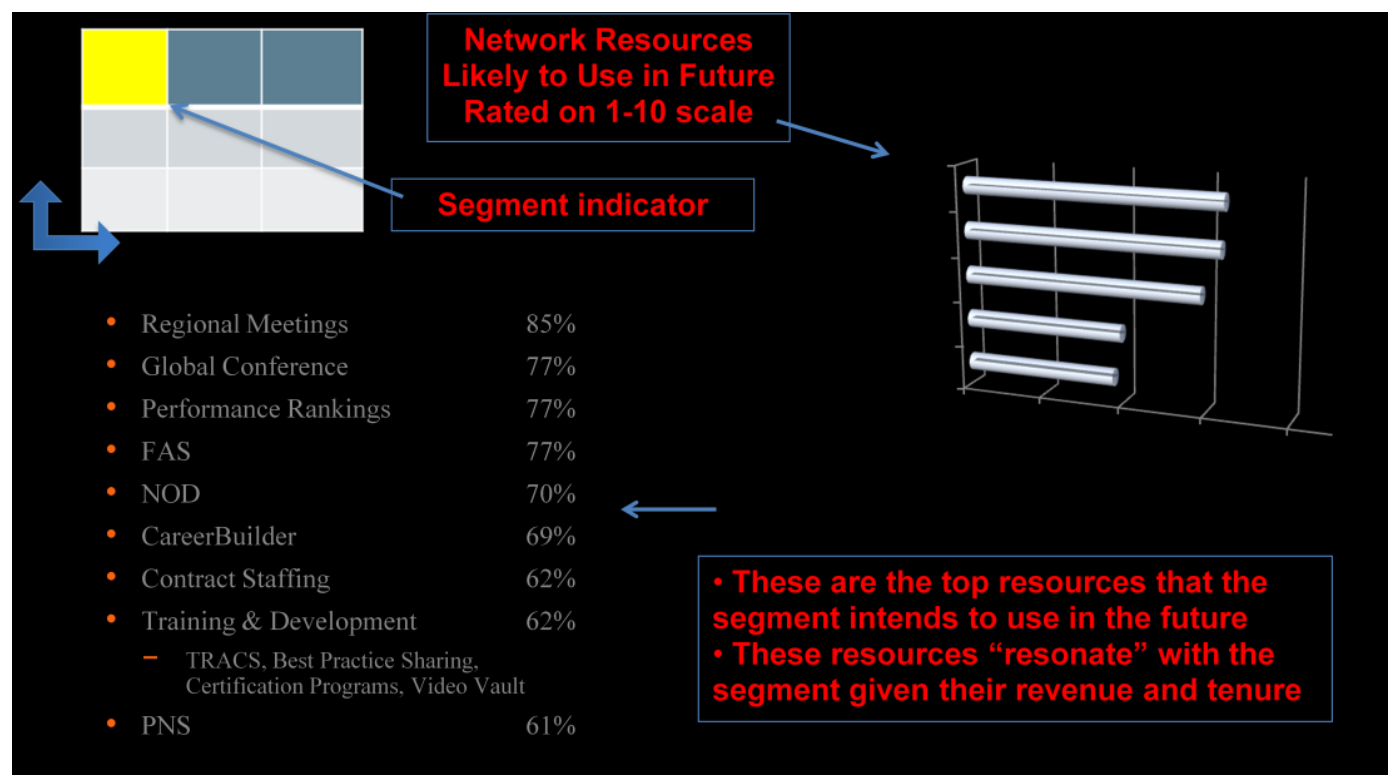

Figure 1.4.2 How to read each of the upcoming figures for resource requirements by segment

\section{Shooting Stars - Low Tenure, High Revenue}

This stellar group had risen quickly with low tenure, less than 8 years with the franchise and reporting high annual revenues, over \$1 million. They reported being involved in the franchise network meetings, and practiced benchmarking with performance rankings. They intended to continue building on their initial training received from the franchisor (NOD is "new office development").

Shooting Stars typically intended to use or continue to use a franchisor program called "Contract Staffing" as a revenue generating resource. This version of recruiting is a bit different in that the recruit is hired as a contractor to the employing company, but actually works for the franchisee who takes a percentage of the hourly fee.

This high-level segment intended to use varied training and development resources, including a support group program (PNS) which encourages open dialog and information exchange among new franchisees. The segment would continue to use the website information resource and wanted field consulting expertise (Figure 1.4.3). 


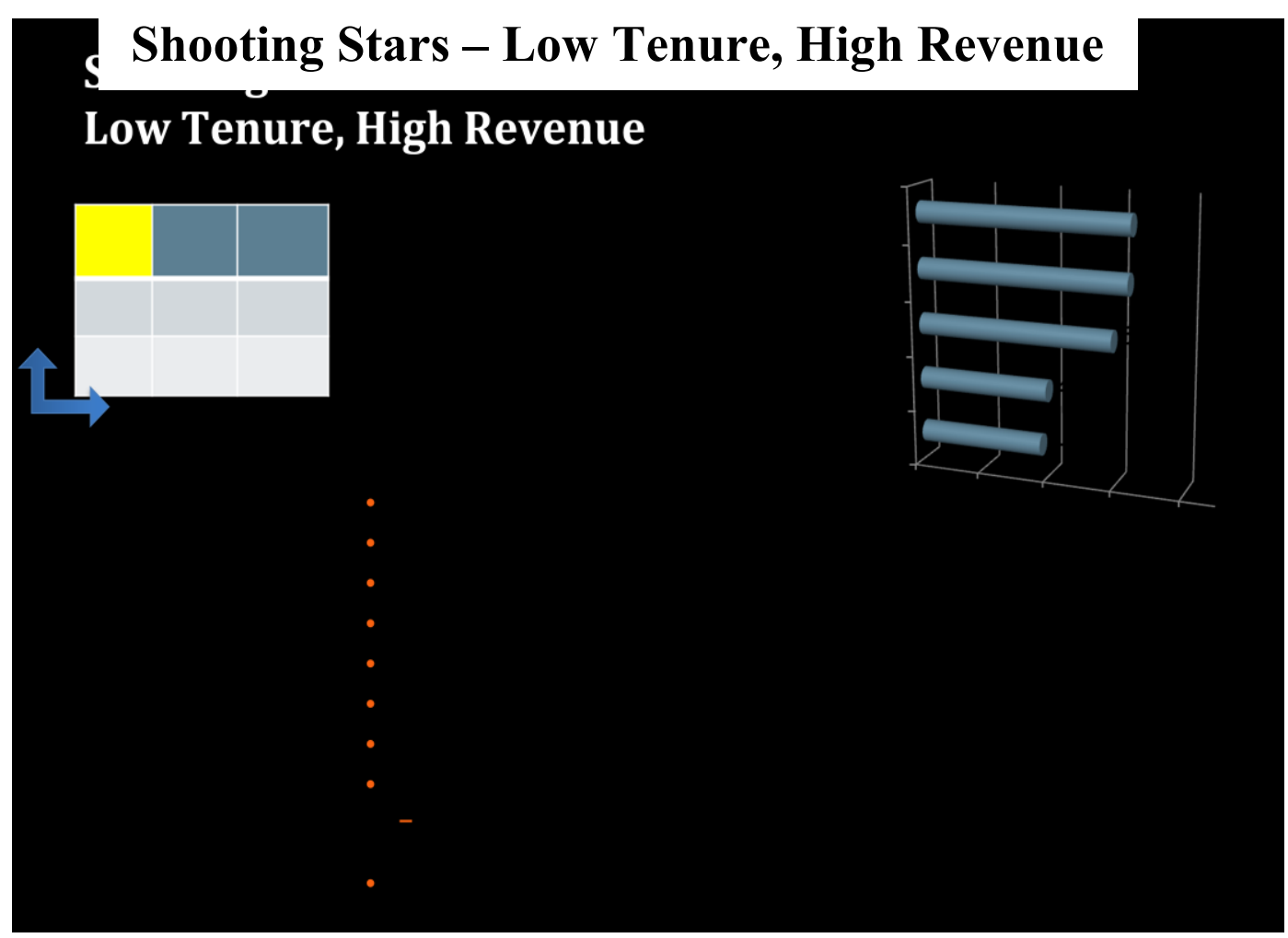

Figure 1.4.3 Low tenure, high revenue segment - franchisor resources required

In the upcoming discriminant analysis, this group will primarily be compared with their low tenure yet low revenue counterparts, the Brat Pack. Thus this segment with low tenure and revenue is discussed next.

\section{Brat Pack - Low Tenure, Low Revenue}

The Brat Pack joined the franchise within the previous 8 years, and had reported annual sales under $\$ 500,000$. These new kids on the block intended to continue participating in franchisor-sponsored events, and intended to continue using a new best practices video series as well as older training tapes (from the Video Vault). This group was open to franchisor training guidance and communication as well as its recommended vendors and technology. Future field consulting was reportedly important to this group, which was open to new offerings such as a training program for new recruiters and interoffice referrals (TRACS and IOR) to continue their own development (Figure 1.4.4) 


\section{Brat Pack - Low Tenure, Low Revenue}

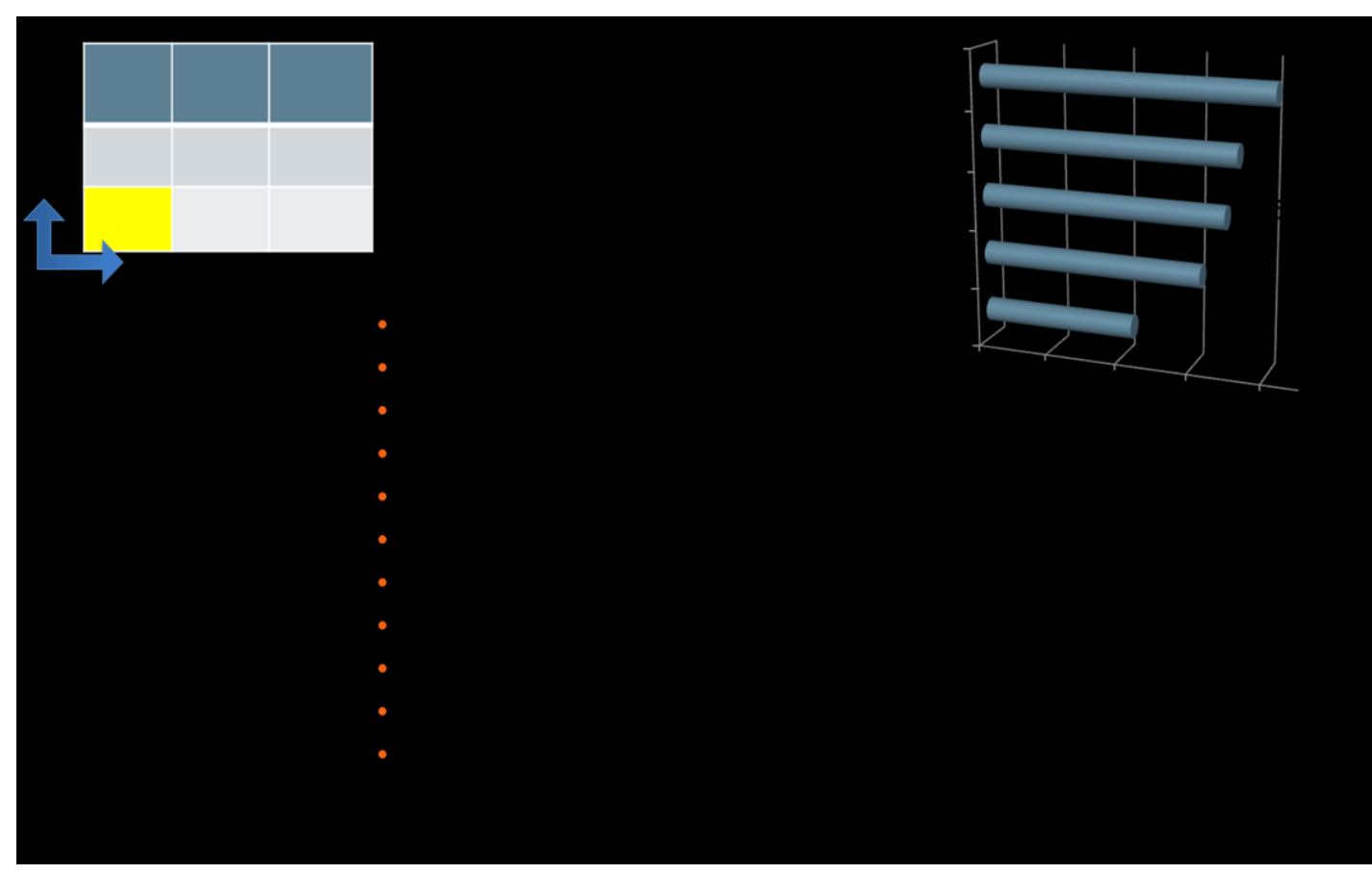

Figure 1.4.4 Low tenure, low revenue segment - franchisor resources required

As mentioned earlier, the key question is, "What can we learn about the differences between higher revenue performers versus lower revenue performers, with the same tenure, that can help the franchisor evaluate needs and allocate resources more effectively?"

Consider the significant differences between these two groups, as indicated by t-tests for significance of difference between their means on the continuous variables measured. Statistically significant differences between these two low tenure segments include overall satisfaction with the franchisor, value in sharing best practices, value perceived in archival training videos, and value perceptions regarding franchise regional meetings. Franchisees with higher revenues rated these resources significantly lower than their lower revenue counterparts of similarly short tenure (Table 1.4.1). Regional meetings are designed to provide hands-on workshops and training for employees of the franchisee (i.e. employment recruiters). The franchisee incurs hotel accommodation expenses, travel, and meeting expenses. Keep in mind that $85 \%$ of the Shooting Stars (high revenue, low tenure) intended to continue to utilize regional meetings as a resource. This higher-revenue segment of newer franchisees finds limited value in these meetings, rating them at 5.85 on the 10 point scale. This may signal the franchisor that a different approach is required for franchisees at a higher performance level. The "Stars" willingness to 
continue to participate is a positive signal to the franchisor, who remains vulnerable to reduced overall satisfaction (rated at 4.82) if improvements to specific resources such as regional meetings are not addressed.

\begin{tabular}{|c|c|c|c|c|c|c|}
\hline & $\bar{x}_{\text {brats }}$ & $\bar{x}_{\text {stars }}$ & $\begin{array}{l}\text { Mean } \\
\text { diff. }\end{array}$ & $t$ & $\mathrm{df}$ & $p$ \\
\hline $\begin{array}{l}\text { Overall satisfaction with } \\
\text { franchisor }\end{array}$ & 6.32 & 4.82 & 1.49 & 2.32 & 51 & .025 \\
\hline $\begin{array}{l}\text { Value best practice } \\
\text { sharing }\end{array}$ & 8.67 & 6.77 & 1.90 & 2.18 & 13 & .047 \\
\hline Value of archived videos & 7.95 & 7.00 & .95 & 2.03 & 51 & .048 \\
\hline $\begin{array}{l}\text { Value of regional } \\
\text { meetings }\end{array}$ & 7.77 & 5.85 & 1.92 & 2.11 & 14 & .053 \\
\hline
\end{tabular}

\section{Rolling Stones - High Tenure, High Revenue}

This segment, comprised of franchisees with 16 or more years' tenure, and revenues exceeding $\$ 1 \mathrm{MM}$, reported a fairly low satisfaction level at 5.4. This segment represents $18 \%$ of the sample, proportionate to new franchisees at low revenue (Brat Pack at 18\%). This segment is considered by the franchise community as the foundation of the network, with high tenure and revenue, and strong support of existing programs. Communications were reportedly considered top priority, and there was a continued demand for classic training materials from the archived videos as well as newer best practices videos. They practiced benchmarking with system-wide performance rankings, and regional meetings were used to motivate teams. They are likely to participate in the international meeting of franchisees with the franchisor (i.e., global conference). They utilize and operating ratio study published by the franchisor and are open to new training programs for new hires. Field consulting is still needed, yet expertise must be substantial (Figure 1.4.5). 


\section{Rolling Stones - High Tenure, High Revenue}

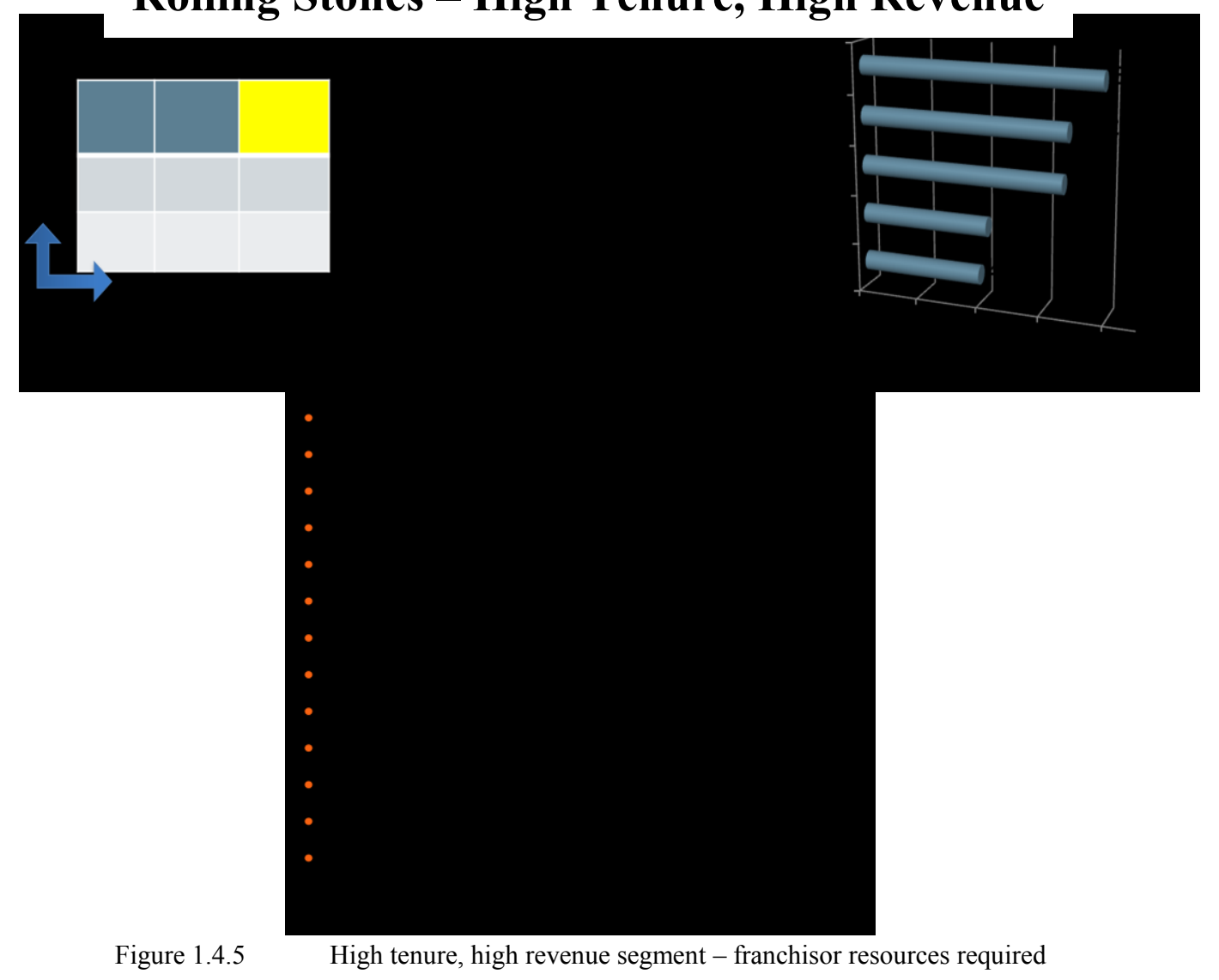

This group is then compared to the high tenure, low revenue group nicknamed Glory Days.

\section{Glory Days - High Tenure, Low Revenue}

This segment has been with the franchise over 15 years yet still reports annual revenues under $\$ 500,000$. Their overall satisfaction with franchisor programs and services is just slightly higher than that of most other segments. Not very interested in field consulting or new programs, this group is loyal to the network vendors, happy to participate in regional meetings, but not exceptionally motivated toward growth at this juncture. 


\section{Glorv Davs - High Tenure. Low Revenue}

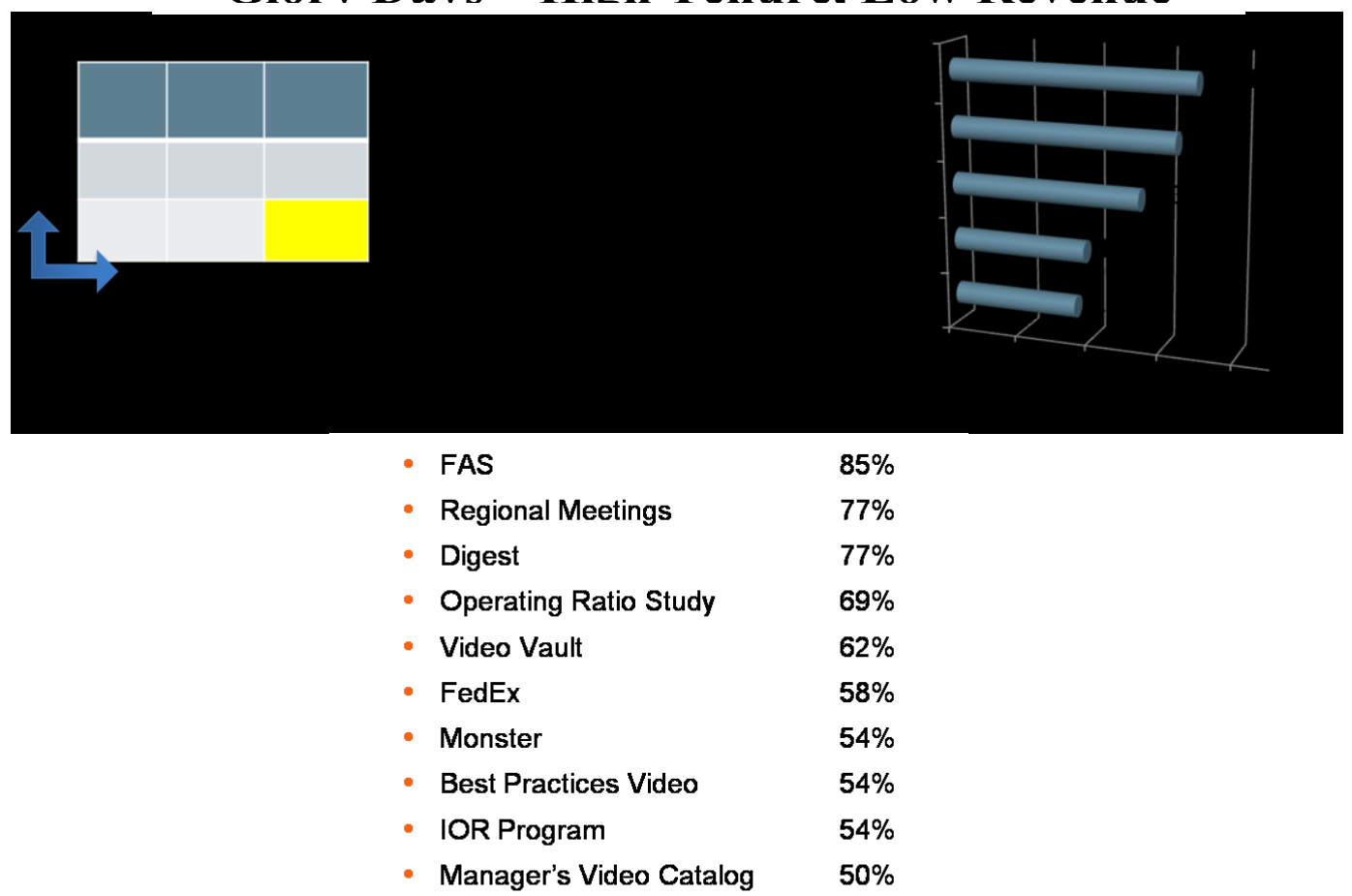

Figure 1.4.6 High tenure, low revenue segment - franchisor resources required

Considering the significant differences between these two high tenure groups, it is found that satisfaction with field consulting services provided by the franchisor, and intent of future use, is significantly higher among the high tenure, high revenue group (Rolling Stones), versus high tenure, low revenue (Glory Days). If the high tenure, low revenue segment is exerting substantial demand on the franchisor's resources, yet rate intent of future use at 4.96, one must discern the potential effectiveness of allocating resources to this high tenure, low revenue segment. Alternately, they may be interested in field consulting, but given the effectiveness of the franchisor's representative (i.e. extent to which the field service representatives help increase sales), dissatisfaction may be leading to low intent of future use.

\begin{tabular}{|l|cccccc|} 
& $\overline{\boldsymbol{X}}_{\text {Ston` }}$ & $\bar{x}_{\text {GloryDays }}$ & $\begin{array}{c}\text { Mean } \\
\text { diff }\end{array}$ & $\mathrm{t}$ & $\mathrm{df}$ & $p$ \\
\hline $\begin{array}{l}\text { Satisfaction with field } \\
\text { consulting }\end{array}$ & 6.75 & 4.53 & 2.21 & 3.45 & 64 & .001 \\
\hline $\begin{array}{l}\text { Intent to } \text { use field } \\
\text { consulting in future }\end{array}$ & 7.59 & 4.96 & 2.57 & 3.58 & 64 & .001 \\
\hline
\end{tabular}

Table 1.4.2 Significant differences between high tenure high revenue (Stones) segment and high tenure, low revenue (Glory Days) segment 


\section{Discussion}

In this section it has been made clear that there are significant differences within the low tenure group that provide insight. Value perceptions regarding regional meetings, best practice sharing, and video archives are all significantly lower among those franchisees in the high revenue category vs. those in the low revenue category. Further, overall satisfaction with the franchisor is significantly lower among the high revenue group as compared to the low revenue group.

The implication is that the franchisor evidences uncertainty and potential risk among the high revenue group of newer franchisees. Renewal of the franchise contract (e.g., at the ten-year mark), is less likely if value perceptions and satisfaction are low.

Franchisees that have been with the system the longest, over sixteen years, and are in the highest revenue category, evidence higher satisfaction with field consulting services provided by the franchisor, than those in the lower revenue category of the high tenure segment. This signals the franchisor to carefully review the low revenue segment's interest in utilizing franchisor resources, particularly in the area of field consulting where satisfaction and future usage intent are significantly lower than among those with higher revenue.

The challenge then, is to identify not only the significant differences between performance levels, but also to identify the variables that will help the franchisor understand a franchisee's propensity to work toward and succeed at a higher level if a focus is placed on utilization of and satisfaction with specific franchisor resources. Discriminant analysis will assist in identifying those variables.

Recall that in the franchise system studied here, the least tenured segment of franchisees had been in the system less than eight years. This group is considered the relative newcomers. The franchisor spends time and resources on training, monitoring, and development this segment. It makes intuitive sense that if the newcomer is encouraged to focus on behaviors reflective of higher performers, particularly with the same level of tenure, then the newcomer is more likely to achieve better results. This research aims at isolating the specific resources used by higher level performers that most differentiate them from their lower performing counterparts, thus providing the franchisor with a tactical guideline for development of franchisees and utilization of resources. 


\subsection{Classification of Franchisees Using Discriminant Analysis}

A logical start to using discriminant analysis to classify franchisees is to use the variables which are known to be significantly different between groups. These variables separate the two groups to a certain extent, yet adding others to the analysis yields better discrimination. This process will be discussed in this section.

\section{Shooting Stars vs. Brat Pack - Discriminant Analysis using Significant Differences}

Recall from Section 1.4, the significant differences between the high (Shooting Stars) and low revenue producers (Brat Pack) in the less tenured group. These included value found in regional meetings, value in sharing best practices, value perceived from viewing archived training videos, and overall satisfaction with the franchisor. In all cases, the higher revenue segment rated these variables significantly lower than their lower revenue counterparts.

Franchisees achieving higher revenue, over $\$ 1$ million, in their early years with the franchisor, less than 8 years, perceived programs and services related to training as less valuable. Regional meetings may not be effectively meeting expectations because the training for newer franchisees is directed toward those with lower revenue. The franchisor might consider training sessions offered by revenue segment instead of tenure. In general, the franchisor's concern is likely to be with overall satisfaction and how to improve it for this higher revenue segment since the royalty stream is more substantial from this group.

Using only the variables with identified significant mean differences in the discriminant analysis, the low tenure group can be classified correctly about $70 \%$ of the time (Table 2.1.1). In the most rigorous test, classification using the holdout sample, the Brats (low tenure, low revenue) were classified correctly $69.2 \%$ of the time and the Stars (low tenure, high revenue) $66.7 \%$ of the time. Recall from Section 2.6 of the Job Seeker analysis, the threshold is $62.5 \%$ for discriminating between two groups.

The discriminant function does a reasonable job differentiating the groups with $\lambda=87$. Recall, Wilks' lambda measures how well each function separates cases into groups. It is equal to the proportion of the total variance in the discriminant scores not explained by differences among the groups. Smaller values of Wilks' lambda indicate greater discriminatory ability of the function. 
Based on the structure matrix (Table 2.1.2), three of the four variables evidence moderate correlation with the discriminant function. These include value of the regional meetings, at .75 , value of best practice sharing at .72 and overall satisfaction with the franchisor at .67. Value regarding the archival training videos did not evidence significant correlation with the discriminant function.

\begin{tabular}{|c|c|c|c|c|c|c|}
\hline \multicolumn{7}{|c|}{ Classification Results $^{\mathbf{a}, \mathbf{b}, \mathbf{c}}$} \\
\hline & & & \multirow[t]{2}{*}{ tenure and revenue } & \multicolumn{3}{|c|}{ Predicted Group Membership } \\
\hline & & & & $\begin{array}{l}\text { Brat Pack } \\
\text { low ten } \\
\text { low rev }\end{array}$ & $\begin{array}{l}\text { Shooting } \\
\text { Stars low } \\
\text { ten hi rev }\end{array}$ & Total \\
\hline \multirow{8}{*}{$\begin{array}{l}\text { Cases } \\
\text { Selected }\end{array}$} & \multirow[t]{4}{*}{ Original } & \multirow[t]{2}{*}{ Count } & Brat Pack low ten low rev & 21 & 6 & 27 \\
\hline & & & $\begin{array}{l}\text { Shooting Stars low ten hi } \\
\text { rev }\end{array}$ & 4 & 6 & 10 \\
\hline & & \multirow[t]{2}{*}{$\%$} & Brat Pack low ten low rev & 77.8 & 22.2 & 100.0 \\
\hline & & & $\begin{array}{l}\text { Shooting Stars low ten hi } \\
\text { rev }\end{array}$ & 40.0 & 60.0 & 100.0 \\
\hline & \multirow{4}{*}{$\begin{array}{l}\text { Cross- } \\
\text { validated }\end{array}$} & \multirow[t]{2}{*}{ Count } & Brat Pack low ten low rev & 21 & 6 & 27 \\
\hline & & & $\begin{array}{l}\text { Shooting Stars low ten hi } \\
\text { rev }\end{array}$ & 5 & 5 & 10 \\
\hline & & \multirow[t]{2}{*}{$\%$} & Brat Pack low ten low rev & 77.8 & 22.2 & 100.0 \\
\hline & & & $\begin{array}{l}\text { Shooting Stars low ten hi } \\
\text { rev }\end{array}$ & 50.0 & 50.0 & 100.0 \\
\hline \multirow{4}{*}{$\begin{array}{l}\text { Cases } \\
\text { Not } \\
\text { Selected }\end{array}$} & \multirow[t]{4}{*}{ Original } & \multirow[t]{2}{*}{ Count } & Brat Pack low ten low rev & 9 & 4 & 13 \\
\hline & & & $\begin{array}{l}\text { Shooting Stars low ten hi } \\
\text { rev }\end{array}$ & 1 & 2 & 3 \\
\hline & & \multirow[t]{2}{*}{$\%$} & Brat Pack low ten low rev & 69.2 & 30.8 & 100.0 \\
\hline & & & $\begin{array}{l}\text { Shooting Stars low ten hi } \\
\text { rev }\end{array}$ & 33.3 & 66.7 & 100.0 \\
\hline \multicolumn{7}{|c|}{ a. $73.0 \%$ of selected original grouped cases correctly classified. } \\
\hline \multicolumn{4}{|c|}{ b. $68.8 \%$ of unselected original grouped cases correctly classified. } & & & \\
\hline $\begin{array}{l}\text { c. } 70.3 \% \text { o } \\
\text { classified. }\end{array}$ & selected cr & s-validat & grouped cases correctly & & & \\
\hline
\end{tabular}

Table 2.1.1 Low tenure franchisees - Classification results from discriminant analysis using only the variables that evidence significant differences between lower tenured franchisees at low revenue vs. high revenue

\begin{tabular}{|l|c|}
\hline \multicolumn{2}{|c|}{ Structure Matrix } \\
\hline \multicolumn{2}{|c|}{} \\
\hline Value regional meetings & Function 1 \\
\hline Value best practices & .749 \\
\hline Overall satisfaction with franchisor & .719 \\
\hline Video vault - archived training videos & .674 \\
\hline
\end{tabular}

Table 2.1.2 Low tenure franchisees - Pooled within-groups correlations between discriminating variables and standardized canonical discriminant function. 


\section{Rolling Stones vs. Glory Days - Discriminant Analysis using Significant Differences}

Next examined is the discriminant function comprised of variables that were determined to be significantly different between the high tenure (16+ years) franchisees with high revenue (Rolling Stones) versus those with low revenue (Glory Days).

Again the Rolling Stones are those with annual revenues over \$1MM, and the Glory Days are those with lower revenue, under $\$ 500,000$ yet the same long tenure as franchisees. Recall that the significant differences between these groups included satisfaction with field consulting services and intent to use field consulting services in the future. In both cases, the higher revenue segment (Rolling Stones) rated field consulting higher than did their lower revenue counterparts (Glory Days).

Using just these two variables to build the discriminant function yields proper classification in about $60 \%$ of the holdout sample cases, with $66.7 \%$ of the Rolling Stones high revenue group correctly classified and $60 \%$ of the Glory Days low revenue group correctly classified (Table 2.1.3).

The discriminant function does a reasonable job differentiating the groups with $\lambda=70$. Based on the structure matrix (Table 2.1.4), both variables evidence strong correlation with the discriminant function. Satisfaction with field services provided by the franchisor offers substantial discrimination in the function, with a coefficient of .90, and the franchisees' intent to use these field services in the future also contributes substantially, with a coefficient of .87 (Table 2.1.5).

Note that the classification results for the holdout sample for the Glory Days segment does not meet the threshold requirement of $62.5 \%$ for discriminating between two groups. 


\begin{tabular}{|c|c|c|c|c|c|c|}
\hline \multicolumn{7}{|c|}{ Classification Results $^{\mathrm{b}, \mathrm{c}, \mathrm{d}}$} \\
\hline & & & \multirow[t]{2}{*}{ tenure and revenue } & \multicolumn{3}{|c|}{ Predicted Group Membership } \\
\hline & & & & $\begin{array}{c}\text { Glory } \\
\text { Days hi } \\
\text { ten low } \\
\text { rev }\end{array}$ & $\begin{array}{c}\text { Rolling } \\
\text { Stones hi } \\
\text { ten hi rev }\end{array}$ & Total \\
\hline \multirow[t]{8}{*}{ Cases Selected } & \multirow[t]{4}{*}{ Original } & \multirow[t]{2}{*}{ Count } & $\begin{array}{l}\text { Glory Days hi ten low } \\
\text { rev }\end{array}$ & 17 & 6 & 23 \\
\hline & & & $\begin{array}{l}\text { Rolling Stones hi ten hi } \\
\text { rev }\end{array}$ & 5 & 20 & 25 \\
\hline & & \multirow[t]{2}{*}{$\%$} & $\begin{array}{l}\text { Glory Days hi ten low } \\
\text { rev }\end{array}$ & 73.9 & 26.1 & 100.0 \\
\hline & & & $\begin{array}{l}\text { Rolling Stones hi ten hi } \\
\text { rev }\end{array}$ & 20.0 & 80.0 & 100.0 \\
\hline & \multirow[t]{4}{*}{$\begin{array}{l}\text { Cross- } \\
\text { validated }\end{array}$} & \multirow[t]{2}{*}{ Count } & $\begin{array}{l}\text { Glory Days hi ten low } \\
\text { rev }\end{array}$ & 17 & 6 & 23 \\
\hline & & & $\begin{array}{l}\text { Rolling Stones hi ten hi } \\
\text { rev }\end{array}$ & 6 & 19 & 25 \\
\hline & & \multirow[t]{2}{*}{$\%$} & $\begin{array}{l}\text { Glory Days hi ten low } \\
\text { rev }\end{array}$ & 73.9 & 26.1 & 100.0 \\
\hline & & & $\begin{array}{l}\text { Rolling Stones hi ten hi } \\
\text { rev }\end{array}$ & 24.0 & 76.0 & 100.0 \\
\hline \multirow[t]{4}{*}{$\begin{array}{l}\text { Cases Not } \\
\text { Selected }\end{array}$} & \multirow[t]{4}{*}{ Original } & \multirow[t]{2}{*}{ Count } & $\begin{array}{l}\text { Glory Days hi ten low } \\
\text { rev }\end{array}$ & 2 & 1 & 3 \\
\hline & & & $\begin{array}{l}\text { Rolling Stones hi ten hi } \\
\text { rev }\end{array}$ & 6 & 9 & 15 \\
\hline & & \multirow[t]{2}{*}{$\%$} & $\begin{array}{l}\text { Glory Days hi ten low } \\
\text { rev }\end{array}$ & 66.7 & 33.3 & 100.0 \\
\hline & & & $\begin{array}{l}\text { Rolling Stones hi ten hi } \\
\text { rev }\end{array}$ & 40.0 & 60.0 & 100.0 \\
\hline \multicolumn{7}{|c|}{ a. $77.1 \%$ of selected original grouped cases correctly classified. } \\
\hline \multicolumn{7}{|c|}{ b. $61.1 \%$ of unselected original grouped cases correctly classified. } \\
\hline \multicolumn{4}{|c|}{ c. $75.0 \%$ of selected cross-validated grouped cases correctly classified. } & & & \\
\hline
\end{tabular}

Table 2.1.3 Highly tenured franchisee segments - Classification results from discriminant analysis using only the variables that evidence significant differences between high tenured franchisees at low revenue vs. high revenue

\begin{tabular}{|l|c|}
\hline \multicolumn{2}{|c|}{ Structure Matrix } \\
\hline & Function 1 \\
\hline $\begin{array}{l}\text { Satisfaction with field } \\
\text { consulting }\end{array}$ & .899 \\
\hline Future use field consulting & .868 \\
\hline
\end{tabular}

Table 2.1.4 Highly tenured franchisee segments - Pooled within-groups correlations between discriminating variables and standardized canonical discriminant function. 


\section{Classification Using Additional Variables}

Next, the aim is to improve these classifications by adding significantly discriminatory variables to the analysis, with the intent of generating a function that yields better classification results. A trial and error method was employed with numerous variables, ultimately resulting in the final discriminant functions and classifications that follow.

\section{Shooting Stars vs. Brat Pack (low tenure segment)}

The final model to discriminate the high and low revenue segments of newer franchisees includes six variables which in future applications can be measured fairly easily. These include:

1. Value of best practices sharing

2. Value of regional meetings

3. Intent to use franchisor's information website

4. Overall satisfaction with franchisor

5. Intent to use franchisor's field consulting services in the future

6. Satisfaction with field consulting services

Value of archival videos was eliminated from the first model because it did not usefully discriminate the groups. Satisfaction with and intent to use field consulting services were added in because they play a prominent role with more tenured franchises. Further, this is a key area of service from the franchisor to franchisees, and requires proper allocation of resources due to its labor-intensive nature.

In building the final model, separate covariance matrices were used because Box's M was significant at $p<001$. The canonical correlation for the discriminant function evidences a moderate relationship between the variables and the function at .56, and Wilkes' lambda is lower, .68, showing improvement from the first model where $\lambda=87$.

The structure matrix suggests that value in best practices, and value found in regional meetings as well as future intent to utilize the franchisor's information website, and overall satisfaction with the franchisor all served to effectively discriminate the groups (Table 2.1.5). 


\begin{tabular}{|l|c|}
\hline \multicolumn{2}{|c|}{ Structure Matrix } \\
\hline & Function 1 \\
\hline Value best practices & .660 \\
\hline Value regional meetings & .583 \\
\hline Future use informational website & .527 \\
\hline Overall satisfaction with franchisor & .478 \\
\hline Future use field consulting services & .134 \\
\hline Satisfaction with field consulting services & .002 \\
\hline $\begin{array}{l}\text { Table 2.1.5 FINAL discriminant function for Low tenure franchisee segments - Pooled } \\
\text { within-groups correlations between discriminating variables and standardized canonical } \\
\text { discriminant function. }\end{array}$
\end{tabular}

Classification is improved with the final model with $85 \%$ of the low-tenure, low-revenue segment classified correctly, and $77 \%$ of the low-tenure, high revenue segment classified correctly (Table 2.1.6).

Given that the validation sets from the first model were used as a basis for improvement, and were acceptable on their own, the validation set is eliminated from the final model because the total number of observations is rather low ( $\mathrm{n}=40$ for Brat Pack and $\mathrm{n}=13$ for Shooting Stars).

\begin{tabular}{|c|c|c|c|c|c|}
\hline \multicolumn{6}{|c|}{ Classification Results $^{a}$} \\
\hline & & \multirow[t]{2}{*}{ tenure and revenue } & \multicolumn{3}{|c|}{ Predicted Group Membership } \\
\hline & & & $\begin{array}{c}\text { Brat Pack low } \\
\text { ten low rev }\end{array}$ & $\begin{array}{c}\text { Shooting } \\
\text { Stars low ten } \\
\text { hi rev }\end{array}$ & Total \\
\hline \multirow[t]{4}{*}{ Original } & \multirow[t]{2}{*}{ Count } & Brat Pack low ten low rev & 34 & 6 & 40 \\
\hline & & $\begin{array}{l}\text { Shooting Stars low ten hi } \\
\text { rev }\end{array}$ & 3 & 10 & 13 \\
\hline & \multirow[t]{2}{*}{$\%$} & Brat Pack low ten low rev & 85.0 & 15.0 & 100.0 \\
\hline & & $\begin{array}{l}\text { Shooting Stars low ten hi } \\
\text { rev }\end{array}$ & 23.1 & 76.9 & 100.0 \\
\hline \multicolumn{5}{|c|}{ grouped cases correctly $\mathrm{cl}$} & \\
\hline
\end{tabular}

Table 2.1.6 Final classification results for low tenure segments using 6 easily measured variables 


\section{Rolling Stones vs. Glory Days (high tenure segment)}

The final model to discriminate the high and low revenue segments of the most tenured franchisees includes eight easily measured variables. These include:

1. Intent to use field consulting services in the future

2. Satisfaction with field consulting services

3. Intent to use franchisor's information website in the future

4. Value in sharing best practices

5. Effectiveness of performance rankings (benchmarking)

6. Overall satisfaction with franchisor

7. Value in performance rankings

8. Value in regional meetings

These variables reflect the discriminators for the lower tenure segment with the addition of effectiveness and value of performance ranking (benchmarking).

The final model results in a canonical correlation of .69 for the discriminant function and $\lambda=53$, both improvements over the model with only satisfaction and future usage intent for field consulting. The structure matrix (Table 2.1.7) suggests however, that these are still the most discriminatory variables, yet the information gained from the other six variables yields better classification as will be discussed.

\begin{tabular}{|l|c|}
\hline \multicolumn{2}{|c|}{ Structure Matrix } \\
\hline & Function 1 \\
\hline Future field consulting & .472 \\
\hline Satisfaction field consulting & .455 \\
\hline Future informational website & .252 \\
\hline Value best practice sharing & -.218 \\
\hline Effectiveness of performance rankings & .096 \\
\hline Overall satisfaction with franchisor & -.062 \\
\hline Value of performance rankings & -.056 \\
\hline Value of regional meetings & -.049 \\
\hline $\begin{array}{l}\text { Table 2.1.7 - FINAL discriminant function for high tenure franchisee segments. } \\
\text { Pooled within groups correlation between discriminating variables and standardized } \\
\text { canonical discriminant function. }\end{array}$
\end{tabular}

canonical discriminant function. 
Classification is improved with the final model with $81 \%$ of the high tenure, low-revenue segment classified correctly, and $85 \%$ of the high tenure, high revenue segment classified correctly (Table 2.1.8).

Given that the validation sets from the first model were used as a basis for improvement, and were acceptable on their own, the validation set is eliminated from the final model because the total number of observations is rather low with $n=26$ for Glory Days (low revenue), and $n=40$ for Rolling Stones (high revenue).

\begin{tabular}{|c|c|c|c|c|c|}
\hline \multicolumn{6}{|c|}{ Classification Results $^{a}$} \\
\hline & & \multirow[t]{2}{*}{ tenure and revenue } & \multicolumn{3}{|c|}{ Predicted Group Membership } \\
\hline & & & $\begin{array}{l}\text { Glory Days hi } \\
\text { ten low rev }\end{array}$ & $\begin{array}{c}\text { Rolling } \\
\text { Stones hi ten } \\
\text { hi rev }\end{array}$ & Total \\
\hline \multirow[t]{4}{*}{ Original } & \multirow[t]{2}{*}{ Count } & $\begin{array}{l}\text { Glory Days hi ten low } \\
\text { rev }\end{array}$ & 21 & 5 & 26 \\
\hline & & $\begin{array}{l}\text { Rolling Stones hi ten hi } \\
\text { rev }\end{array}$ & 6 & 34 & 40 \\
\hline & \multirow[t]{2}{*}{$\%$} & $\begin{array}{l}\text { Glory Days hi ten low } \\
\text { rev }\end{array}$ & 80.8 & 19.2 & 100.0 \\
\hline & & $\begin{array}{l}\text { Rolling Stones hi ten hi } \\
\text { rev }\end{array}$ & 15.0 & 85.0 & 100.0 \\
\hline
\end{tabular}

Table 2.1.8 Final classification results for high tenure segments using 8 easily measured variables. 


\section{Densities Based on Discriminant Scores by Class}

Consider the assigned groupings for both the low and high tenure segments. Comparing the discriminant scores of Shooting Stars vs. Brat Pack, separation between segments is clear (Figure 2.1.1), as is that of Rolling Stones vs. Glory Days (Figure 2.1.2).

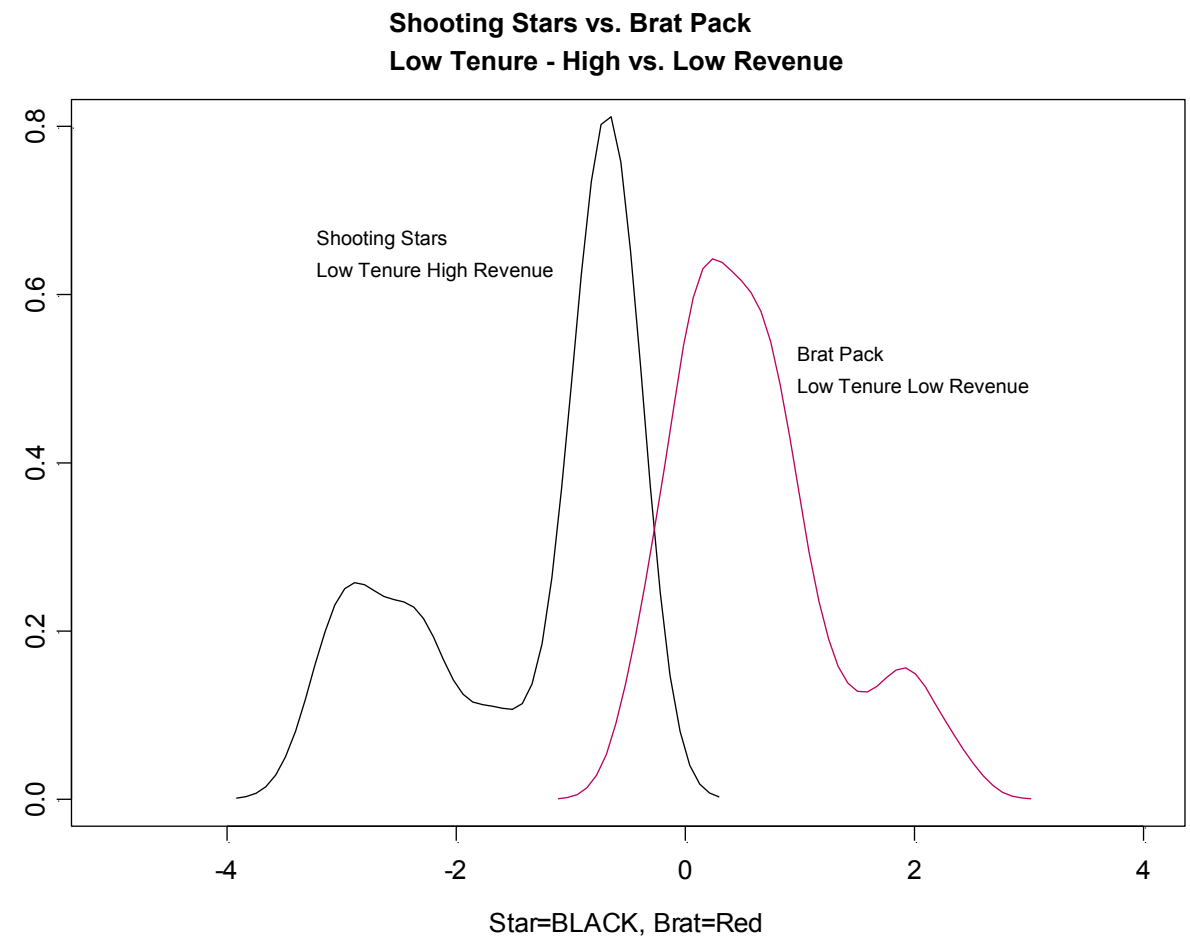

Figure 2.1.1 Discriminant scores for low tenure segment based on group classification 
Rolling Stones vs. Glory Days

High Tenure - High vs. Low Revenue

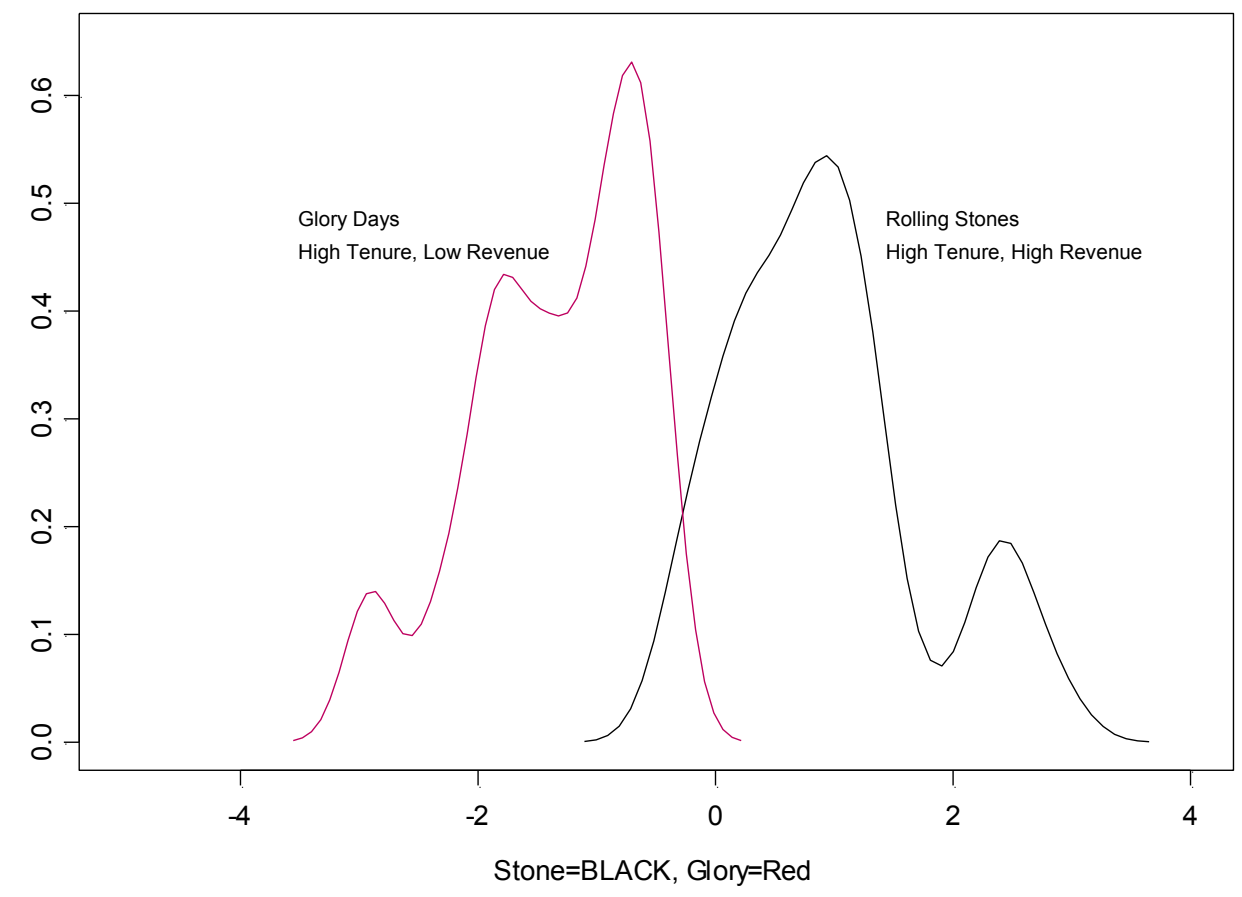

Figure 2.1.2 Discriminant scores for high tenure segment based on group classification 


\subsection{Summary - Classification and Analysis of Franchise Resource Data}

In the first portion of this study, the focus was on newer franchisees. The discriminating variables suggest lower satisfaction and value in the services provided by the franchisor within the higher revenue segment compared to the lower revenue. The suggested franchisor response is to tailor services to more effectively address the needs of the segment which earns higher revenues, thus higher royalties are paid to the franchisor. This action would reduce the franchisor's risk of the high-revenue new franchisee not renewing their franchise agreement due to dissatisfaction with value provided by franchisor.

In the second situation, where the focus was on the most tenured franchisees, the higher revenue segment is significantly more satisfied with the franchisor's field consulting services. Thus, resources should be allocated to maintain these satisfaction levels.

Classification of a franchisee into one of these segments is facilitated by discriminant analysis, with an accuracy of over $80 \%$. Applying the technique, one can generate scores for as few as eight variables and determine fairly well the potential revenue segment of a franchisee for a current year. The franchisor's field consultants should focus on resources used by the higher revenue segments (e.g., benchmarking with performance rankings) to guide the lower revenue segments, particularly as tenure increases.

The management issue addressed was allocation of resources to most effectively support franchisee revenue growth. The most effective classification and analytic method was discriminant analysis to understand the resources that discriminate revenue performance groups. The contribution is the presentation of a tool for effectively allocating franchisor resources which mimics the resource utilization behaviors of the highest revenue-generating categories.

The approach taken here supports Ananad's (1987) suggestion that management consider using subsamples to influence lower performers to act like their higher performing counterparts. However, the practical applications may require varied forms. For example, given the dataset investigated here, the least tenured group was considered the relative newcomers. This group provided feedback on services they were currently receiving, within the franchise system, from the franchisor. If the franchisor wanted to classify "potential" franchisees, say those who were investigating the option of buying a franchise (i.e., becoming a franchisee), they could not provide comparable usage, effectiveness, value and satisfaction data because they would not have yet used the services. However, this tool can be used as a directional guide for 
those within the system, yet for a short period of time. The focus of servicing the low revenue group should be encouraging the service-usage behaviors of their higher revenue counterparts with equal tenure in the franchise system.

Alternately, a screening interviewer for the franchisor might discuss the potential franchisee's likelihood to use certain services which are reflective of the higher performers' preferences. For example, would the potential franchisee be willing to participate in regional meetings, benchmarking, and sharing of best practices?

The value to the franchisor is in isolating the behaviors (in the form of services used), that are most likely to spawn revenue growth. Also, a potential franchisee can more clearly identify the requirements of success, and make a more informed decision regarding purchasing a franchise. Analytic tools such as those discussed here can be designed specifically for a particular franchise system. Such tools can aid in reducing conflict regarding marketing services provided by the franchisor to the franchisee. 


\section{References Classification and Analysis of Franchise Resource Data}

Aksoy, L. A. (2008). The long-term stock market valuation of customer satisfaction. Journal of Marketing 72, 105-22.

Anand, Punam, and Louis W. Stern. (1985). A sociopsychological explanation for why marketing channel members relinquish control. Journal of Marketing Research 22, 365-76.

Anand, Punam. (1987). Inducing franchisees to relinquish control: An attribution analysis. Journal of Marketing Research 24 (2), 215-21.

Connor, P. E. \&. Bloomfield, S. D. (1977). A goal approach to organizational design. TIMS Studies in the Management Sciences 5, 99-110.

Dant, R. P. \& P. D. Berger. (1996). Modeling cooperative advertising decisions in franchising. Journal of the Operational Research Society 47 (9), 1120-136.

Doyle, P. (2000). Value-based marketing; Marketing strategies for corporate $g$ and shareholder value. Chichester, UK: John Wiley \& Sons.

Eliashberg, J. \& Michie, D. A. (1984). Multiple business goals sets as determinants of marketing channel conflict: An empirical study. Journal of Marketing Research 21 (1), 75-88.

Glosband, B. A. (1972). The franchising dilemma. Trial 8, 34.

Hunt, S. D. \& Nevin, J. R. (1975). Tying agreements in franchising. Journal of Marketing 39 (3), 20-26.

Hunt, S. D. (1972). The socioeconomic consequences of the franchise system of distribution. Journal of Marketing 36 (3), 32-38.

Kalnins, A. (2004). An empirical analysis of territorial encroachment within franchised and company-owned branded chains. Marketing Science 23 (4), 476-89.

Lafontaine, F. \& Shaw, K. L. (2005). Targeting managerial control: Evidence from franchising. RAND Journal of Economics 36. (1), 131-50.

Lal, R. (1990). Improving channel coordination through franchising. Marketing Science 9 (4), 299-318. 
Lillis, C. M., Narayana, C. L. \& Gilman, J. L. (1976). Competitive advantage variation over the life cycle of a franchise. Journal of Marketing 40 (4), 77-80.

Luo, X. (2008). Satisfaction, complaint, and the stock-value gap. Journal of Marketing 72, 29-43.

Lusch, R. F. \& Brown, J. R. (1982). A modified model of power in the marketing channel. Journal of Marketing Research 19 (3), 312-23.

Lusch, Robert F. (1976): Sources of Power: Their Impact on Interchannel Conflict. Journal of Marketing Research 13 (4), 382-90.

Michael, S. C. (1996). To franchise or not to franchise: An analysis of decision rights and organizational form shares. Journal of Business Venturing 11, 57-71.

Nault, B. R. (1995). Adoption, transfers, and incentives in a franchise network with positive externalities. Marketing Science 13 (4), 412-23.

Norton, S. W. (1988). Franchising, brand name capital, and the entrepreneurial capacity problem. Strategic Management Journal 9, Special issue: Strategy content research, 105-14.

Perrow, C. (1970). Organizational analysis: A sociological analysis. Belmont, CA: Brooks/Cole.

Rao, R. K. (2008). Marketing initiatives, expected cash flows, and shareholders' wealth. Journal of Marketing 72, 16-26.

Rust, R. T., Ambler, T., Carpenter, G. S., Kumar, V. \& Srivastava, R. K. (2004). Measuring marketing productivity: Current knowledge and future directions. Journal of Marketing 68, 76-89.

Schul, P. L., Pride, W. M. \& Little, T. L. (1983). The impact of channel leadership behavior on interchannel conflict. Journal of Marketing 47 (3), 21-34.

Seshadri, S. (2002). Outlet ownership in franchising systems: An agency based approach. Managerial and Decision Economics 23 (6), 355-69.

Srivastava, R. K. (1998). Market-based assets and shareholder value: A framework for analysis. Journal of Marketing 62, 2-18.

Wittreich, W. J. (1962). Misunderstanding the retailer. Harvard Business Review 40, 147-59. 
Appendix A - Classification and Analysis of Job-Seeking Market

Section 2.3 - Descriptive Statistics for Factors and Loyalty Scores by Subclass

The Kruskal-Wallis test is a nonparametric method for testing the equality of population medians among group. The results suggest that the medians of the rank ordered groups are significantly different. However, the test assumes identically shaped and scaled distributions, and it is known that the nonGaussian distributions have unequal variance. A focus was placed instead on the asymptotic normality of the $t$ statistic and its usefulness applied to the jobseeker dataset.

\begin{tabular}{|l|l|r|r|}
\hline & $\begin{array}{l}\text { Seeker } \\
=1 \\
\text { Passiv } \\
\mathrm{e}=2 \\
\text { Non=3 }\end{array}$ & \multicolumn{1}{|c|}{$\mathrm{N}$} & Mean Rank \\
\hline \multirow{5}{*}{ SUPV } & 1 & 1193 & \\
\cline { 2 - 4 } & 2 & 451 & 1017.92 \\
\cline { 2 - 4 } & 3 & 102 & 1238.91 \\
\cline { 2 - 4 } & Total & 1746 & \\
\hline COMP & 1 & 1163 & 725.27 \\
\cline { 2 - 4 } & 2 & 442 & 1079.28 \\
\cline { 2 - 4 } & 3 & 100 & 1338.32 \\
\cline { 2 - 4 } & Total & 1705 & \\
\hline \multirow{5}{*}{ OPPTY } & 1 & 1210 & 914.46 \\
\cline { 2 - 4 } & 2 & 451 & 828.57 \\
\cline { 2 - 4 } & 3 & 100 & 712.56 \\
\cline { 2 - 4 } & Total & 1761 & \\
\hline \multirow{5}{*}{ QWL } & 1 & 1193 & 920.27 \\
\cline { 2 - 4 } & 2 & 455 & 791.06 \\
\cline { 2 - 4 } & 3 & 101 & 718.36 \\
\cline { 2 - 4 } & Total & 1749 & \\
\hline
\end{tabular}

\begin{tabular}{|c|c|c|c|c|}
\hline & SUPV & COMP & OPPTY & QWL \\
\hline Chi-Square & 125.25 & 268.82 & 21.26 & 31.87 \\
\hline $\mathrm{df}$ & 2 & 2 & 2 & 2 \\
\hline Asymp. Sig. & .000 & .000 & .000 & .000 \\
\hline
\end{tabular}

\begin{tabular}{|l|c|c|c|}
\hline & Subclass & $\mathrm{N}$ & Mean Rank \\
\hline \multirow{4}{*}{ Loyalty } & 1 & 1058 & 660.91 \\
\cline { 2 - 4 } & 2 & 411 & 982.91 \\
\cline { 2 - 4 } & \multicolumn{1}{|c|}{3} & 93 & 1263.24 \\
\cline { 2 - 4 } & Total & 1562 & \\
\hline & & & \\
\hline $\begin{array}{l}\text { Chi- } \\
\text { Square }\end{array}$ & 263.651 & & \\
\hline df & 2 & & \\
\hline $\begin{array}{l}\text { Asymp. } \\
\text { Sig. }\end{array}$ & .000 & & \\
\hline
\end{tabular}


Appendix B - Classification and Analysis of a Job-Seeking Market

Section 2.6 Discriminant Analysis - Validation test results

Classification Results ${ }^{b, c, d}$

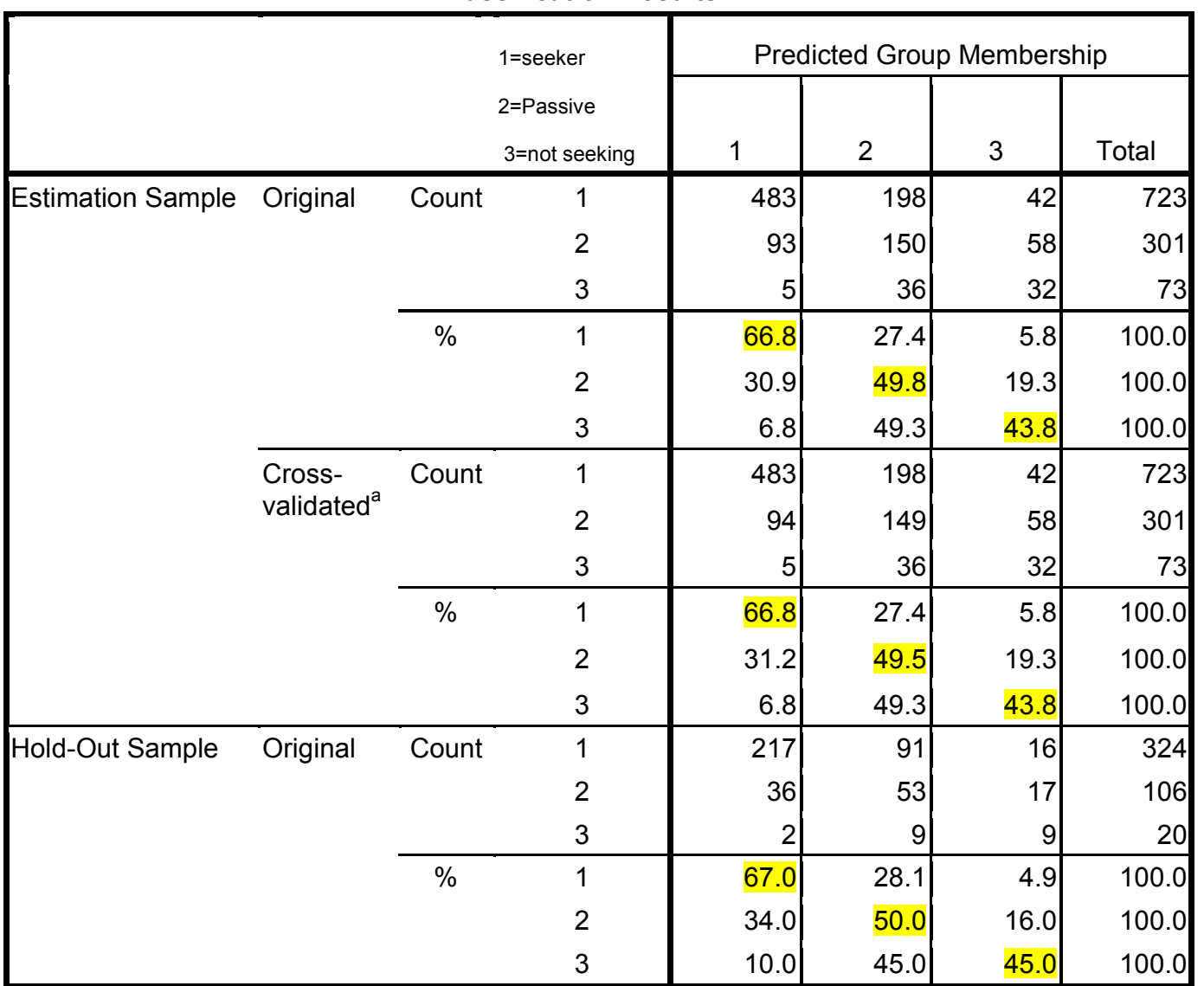

a. Cross validation is done only for those cases in the analysis. In cross validation, each case is classified by the functions derived from all cases other than that case.

b. $60.6 \%$ of estimation sample cases correctly classified.

c. $62.0 \%$ of hold-out sample cases correctly classified.

d. $60.5 \%$ of estimation sample cross-validated grouped cases correctly classified. 
Validate 2

Classification Results ${ }^{a, b, c}$

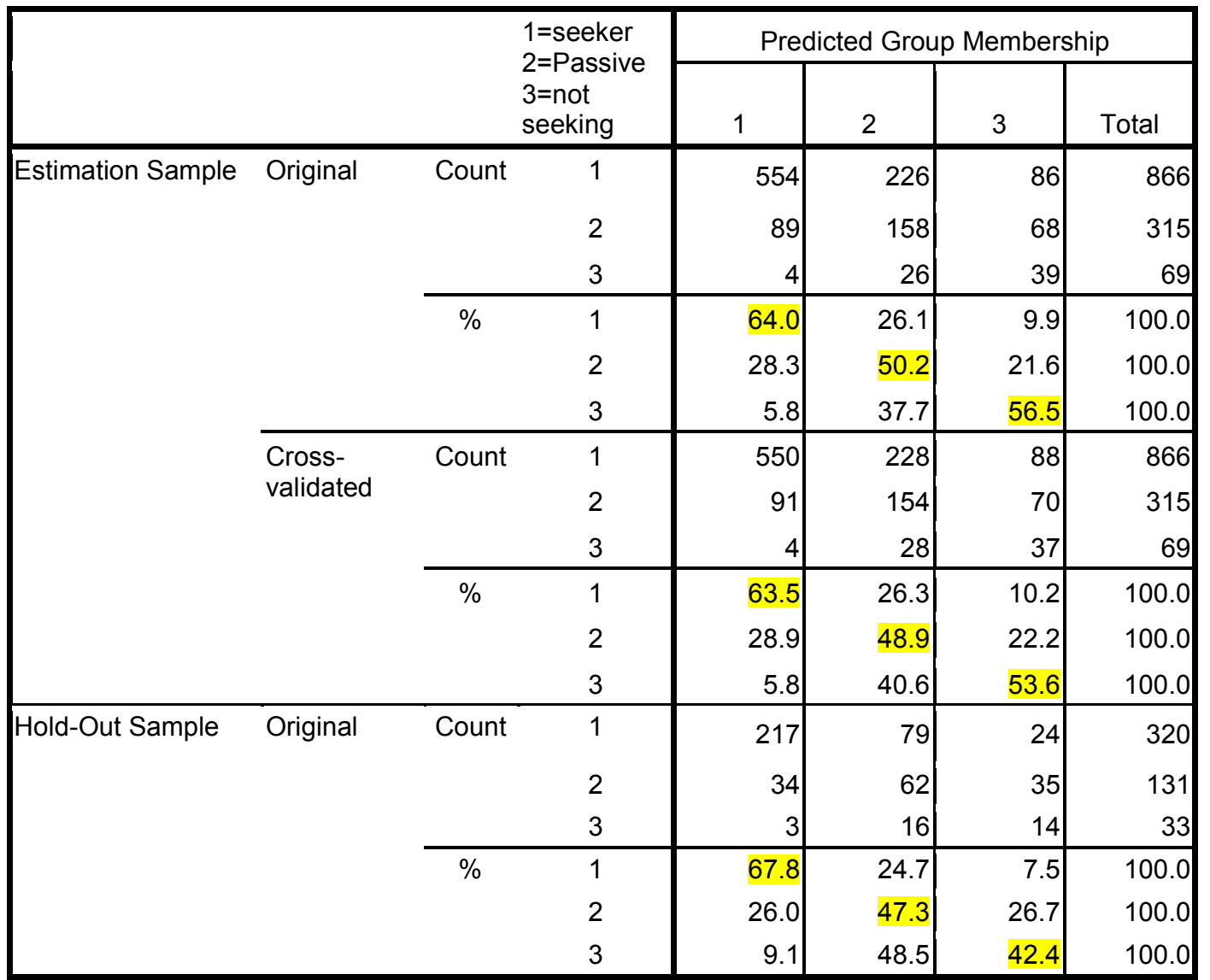

a. $60.1 \%$ of estimation cases correctly classified.

b. $60.5 \%$ of holdout cases correctly classified.

c. $59.3 \%$ of estimation cross-validated grouped cases correctly classified. 
Validate 3

Classification Results ${ }^{a, b, c}$

\begin{tabular}{|c|c|c|c|c|c|c|c|}
\hline & & & $1=$ seeker & Prec & d Grou & lembers & \\
\hline & & & $\begin{array}{l}\text { z=rassive } \\
3=\text { not seeking }\end{array}$ & 1 & 2 & 3 & Total \\
\hline Estimation Sample & Original & Count & 1 & 529 & 201 & 77 & 807 \\
\hline & & & 2 & 88 & 137 & 94 & 319 \\
\hline & & & 3 & 2 & 19 & 41 & 62 \\
\hline & & $\%$ & 1 & 65.6 & 24.9 & 9.5 & 100.0 \\
\hline & & & 2 & 27.6 & 42.9 & 29.5 & 100.0 \\
\hline & & & 3 & 3.2 & 30.6 & 66.1 & 100.0 \\
\hline & Cross- & Count & 1 & 524 & 206 & 77 & 807 \\
\hline & validated & & 2 & 89 & 136 & 94 & 319 \\
\hline & & & 3 & 2 & 22 & 38 & 62 \\
\hline & & $\%$ & 1 & 64.9 & 25.5 & 9.5 & 100.0 \\
\hline & & & 2 & 27.9 & 42.6 & 29.5 & 100.0 \\
\hline & & & 3 & 3.2 & 35.5 & 61.3 & 100.0 \\
\hline Holdout sample & Original & Count & 1 & 241 & 95 & 38 & 374 \\
\hline & & & 2 & 36 & 61 & 33 & 130 \\
\hline & & & 3 & 2 & 12 & 25 & 39 \\
\hline & & $\%$ & 1 & 64.4 & 25.4 & 10.2 & 100.0 \\
\hline & & & 2 & 27.7 & 46.9 & 25.4 & 100.0 \\
\hline & & & 3 & 5.1 & 30.8 & 64.1 & 100.0 \\
\hline
\end{tabular}

a. $59.5 \%$ of estimation cases correctly classified.

b. $60.2 \%$ of holdout cases correctly classified.

C. $58.8 \%$ of estimation sample cross-validated grouped cases correctly classified. 
Die Dissertation hat folgende Erklärung zu enthalten:

"Ich versichere an Eides Statt, dass ich die eingereichte Dissertation "Classification and Analysis of Management and Marketing Data: Statistical Applications for Strategic Planning" selbstständig verfasst habe. Anderer als der von mir angegebenen Hilfsmittel und Schriften habe ich mich nicht bedient. Alle wörtlich oder sinngemäß den Schriften anderer Autorinnen und/oder Autoren entnommenen Stellen habe ich kenntlich gemacht."

Signature

11 March 2010

Date 
Tina M. Facca

March,2010

Citizenship: United States of America

Email:tfacca@jcu.edu

\section{Education:}

2009 Master of Science, Statistics, Case Western Reserve University 1994 Master of Applied Communication Theory \& Methodology, Cleveland State University

1987 Bachelor of Arts, Communication, Cleveland State University

\section{Teaching Experience:}

- Fall 2008 to present, Tenure-track faculty, John Carroll University, Boler School of Business, Department of Management, Marketing and Logistics (Cleveland, Ohio, USA)

- Market Analysis, Marketing Principles, Marketing Management

- Wassmer Grant for paper analyzing empirical research with job seekers

- Wassmer Grant for paper on franchising resource allocation

- 2004-2006, Adjunct Faculty, Case Western Reserve University, School of Engineering

- Graduate course focusing on applied statistics for new product development, evaluation of markets, and product management

- 2003, Adjunct Faculty John Carroll University, Boler School of Business; Management, Marketing \& Logistics department,

- Undergraduate course in Marketing Decision Support Systems including software review, product feasibility research, product development and management

- Graduate course in Quantitative Methods with applications including optimization modeling, network flow models, non-linear optimization models and multi-objective decision making

- 2002 -2004, Adjunct Faculty, Cleveland State University, James J. Nance School of Business Administration; Operations Management and Statistics

- Graduate courses in Statistical Business Decisions applying statistical methods via traditional mathematics and statistical software packages

- Undergraduate Statistics for Business and Economics I \& II; practical applications of statistics utilizing analytical tools available to solve typical business problems and develop business strategies. Topics included inferences about population variances, chi-squared tests for independence, analysis of variance, simple and multiple linear regression analysis.

\section{Conference Presentations:}

Bridging the gap between marketing and finance in undergraduate curriculum. International Conference on Education Research and Innovation 2009, Madrid, Spain

Classification and analysis of job-seeking behaviors. International Decision Sciences Institute 2009, Nancy, France 


\section{Publications:}

Shankman, M. L., Allen, S. \& Facca, T. M. (2010). Emotionally intelligent leadership inventory. San Franisco: Jossey Bass.

\section{Industry Employment Experience:}

15+ years high level marketing management, new product development and management, and consumer market research and analysis.

May 1990 to Present, The Foresight Group, President; Marketing and management consulting, specializing in research for strategic business planning.

January 2007 to March 2008: Management Recruiters International, Inc., Vice President of Marketing, for $\$ 560 \mathrm{MM}, 100 \%$ franchised network of executive recruiting firms with 1100 offices in 40 countries.

September 2005 to January 2007: TravelCenters of America, Inc., Director of Marketing for $\$ 200 \mathrm{MM}$ system of travel plazas, $20 \%$ franchised; Oversight of restaurant marketing for 140 unit family dining chain inclusive of product development function, introducing nearly 100 new menu items

September 2003 to September 2005, Vita-Mix Corporation, Product Manager for \$70MM blending equipment manufacturer; Responsible for successful product launch through detailed product development process; Responsible for the development and maintenance of systems to guide product initiatives through development process

October 2000 to March 2001, Technology Strategies, Vice President of Marketing \& Communications, for application service provider of on-line event registration system

August 1998 to October 2000, Damon's International, Inc. Vice President of Marketing for $\$ 300 \mathrm{MM}$ privately-held, international casual dining chain of 135 restaurants; $80 \%$ franchised

April 1993 to May 1994, Benesch, Friedlander, LLP. Marketing Research Coordinator for Cleveland based law firm

March 1988 to April 1990 TRW Real Estate Loan Services; Marketing Research Analyst for multi-unit provider of loan services to the real estate industry 
My deep gratitude is expressed to Professor Dr. Walter Zucchini for his consistent patience and foresight during the development of this research. His elegant simplicity in expression and presentation of complicated statistical concepts not only shed new light but ignited many fires. I am equally grateful to Professor Dr. Manfred Denker for identifying the potential inside of me, and carefully extracting it with clarity and definition. His constant willingness to work through mathematical details in as many ways as necessary to guarantee comprehension has been an inspiration to my own teaching. I am deeply grateful to Professor Dr. Wojbor Woyczynski for his initial encouragement and consistent guidance through the challenges related to academic growth and accomplishment. Many thanks also to my examiners Professor Dr. Yasemin Boztug and Professor Dr. Stefan Sperlich. Immense gratitude to my mom, Rita, who traveled far beyond her geographic comfort zone to support this endeavor because she knew how proud my dad would be if he were still here to share in this. His spirit survives, and the deep roots of faith he planted in me will continue to bear fruit. I am intensely grateful to my beloved John D. Miess, who patiently endured every challenge with me and insightfully provided suggestions and solutions with the end always in sight. This accomplishment is clearly the result of his unwavering encouragement, discipline, support, and love. And thank you Lord, for my constant prayer:

I can do all things through Him who gives me strength. Philippians 4:13 